\title{
A decision model for the investment in technology to reduce concrete rework
}

\author{
by \\ Tian Martin Podges
}

Thesis presented in fulfilment of the requirements for the degree of Master of Engineering in Civil Engineering in the Faculty of Engineering at Stellenbosch University

Supervisor: Prof. Jan Andries Wium

Co-supervisor: Chris Jurgens 


\section{Declaration}

This thesis is a presentation of my original research work. Wherever contributions of others are involved, every effort is made to indicate this clearly, with due reference to the literature, and acknowledgement of collaborative research and discussions.

The work was done under the guidance of Professor J.A. Wium, at the University of Stellenbosch, South Africa.

Tian Martin Podges

Date

In my capacity as supervisor of the candidate's thesis, I certify that the above statements are true to the best of my knowledge.

Prof. J.A. Wium

Date 


\section{Abstract}

This study investigated a method to choose technology that reduces the rework of structural concrete works which results in poor quality during construction. The technology considered is identified as technology that can be used to improve quality control during construction. The scope of the investigation was limited to construction that consists of structural elements, such as beams, columns, slabs and concrete walls. The research questions for this study were as follows: (i) what are the causes that lead to poor construction quality of structural concrete; (ii) how can technology be used to improve the construction quality and how effective would it be and (iii) can a decision model be developed to assist a contractor to invest in such technology.

The motivation pertaining to this study was based on the excessive rework costs experienced on construction projects. Rework has a disadvantageous influence on both cost and time of a project. In structural concrete projects, direct rework costs were found to be between $4-6 \%$ of the total value of concrete works. Literature showed that barriers toward obtaining good construction quality are identified as attributes of labour, management, subcontractors, and plant and equipment. The construction errors that are caused as a result of these attributes were identified and are seen as risks that could lead to rework.

A questionnaire survey was used to determine the influence of labour-, management-, subcontractors-, and plant and equipment attributes on the quality of structural concrete. Furthermore, it was also used to determine the influence of these categories on the construction errors that were identified. Interviews were conducted to determine measures, pertaining to the identified construction errors, that could be implemented to prevent construction rework. These measures were used in the study as important processes to improve the quality control of concrete. Moreover, it was useful to establish a criteria when evaluating the effectiveness of technology to reduce construction errors. A range of technological systems, such as laser scanners, dimensioning software applications, internet protocol cameras, radio frequency identification tags, quick response codes and concrete curing sensors were evaluated for its capability and effectiveness to monitor the construction activities where construction errors potentially occur.

The information gathered regarding construction barriers, -errors and technology effectiveness was used in a decision model based on risk and cost. The model advises the user whether an investment should be made to acquire technology for a project to reduce construction rework. A fictitious project with various scenarios was investigated. The model presented feasible investment decisions in cases of medium to high risk. The estimated savings for the fictitious project ranged between R 260000 and R 1.17 million 
as the expected risk changed. A sensitivity analysis was conducted to determine how the model suggestion varies for different project sizes and rework expectancies. It was found that the model is very sensitive when expected rework is less than $1 \%$. The model was validated through interviews with experts in the construction industry. 


\section{Opsomming}

In hierdie studie is 'n metode ondersoek om konstruksieherwerk met behulp van tegnologie te verminder. Die tipes konstruksie waarop gefokus word sluit beton strukturele elemente soos balke, kolomme, blaaie en mure in. Die ondersoekende vrae in die studie is soos volg: (i) Wat is die oorsake van slegte konstruksiekwaliteit? (ii) Hoe kan tegnologie gebruik word om konstruksie kwaliteit te verbeter en hoe effektief sal dit wees? (iii) Kan 'n besluitnemingsmodel om 'n kontrakteur te help met die aankoop van sulke tegnologie ontwikkel word.

Die motivering van die studie word deur die hoë herwerkkostes in die konstruksie-industrie gedryf. Herwerk het nagevolge met betrekking tot die tyd en koste van 'n projek. In SuidAfrika word herwerkwaardes van tussen $4-6 \%$ op die totale waarde van beton werk op 'n projek ondervind. In die navorsingsbronne word herwerk aan eienskappe van arbeid, bestuur, subkontrakteurs, en aanleg en toerusting toegeskryf.

'n Vraelysopname is gebruik om die invloed van arbeid, bestuur, subkontrakteurs, en aanleg en toerusting op die kwaliteit van konstruksie te bepaal. Dit is ook gebruik om die invloed van hierdie kategorieë op konstruksiefoute te bepaal. Onderhoude is met kenners in die industrie gevoer om vas te stel watter maatreëls gebruik kan word om konstruksiefoute te verminder. Die maatreëls is gebruik in die vestiging van 'n kriteria waaraan tegnologie moet voldoen om vir kwaliteit verbetering gebruik te word. Die volgende tipes tegnologie is oorweeg vir die studie: laserskandeerders, afmeting-sagteware, radio-frekwensie-identifiseringstoestelle, vinnige reaksiekodes en betonsensors.

Die inligting rakende konstruksiehindernisse, foute, voorsorg maatreëls en tegnologie effektiwiteit is in die ontwikkeling van 'n besluitnemingsmodel gebruik. Die model is gerig op risiko en koste: Op grond daarvan word 'n kontrakteur geadviseer of die belegging in tegnologie tot koste besparing kan lei. 'n Fiktiewe projek met verskeie senarios word gesimuleer om die gebruik van die model te demonstreer. Deur die simulasies word beraamde besparings tussen R 260000 en R 1.17 miljoen deur die model voorspel. 'n Sensitiwiteitsanalise is ook gedoen om te wys hoe die beraamde koste besparing wissel vir verskeie projekwaardes en herwerkverwagtinge. Die mening van kenners in die industrie is tydens onderhoude ingewin. Hulle het hul goedkeuring gegee. 


\section{Acknowledgements}

First and foremost, I acknowledge the invaluable guidance of my research supervisor, Prof. J.A. Wium, and the Co-supervisor, Mr C. Jurgens, for their tireless efforts to ensure that this study is completed.

My gratitude extends to all my friends who remained steadfast and supportive. Particular thanks go to Mrs Natalia Buhrmann, Mrs Petro van Der Walt, Mr Wagenaar Human and Mr Moses Malinda for the moral support they bestowed on me to ensure that this piece of work becomes a great success.

I would like to register my deep appreciation and thanks to my family. First, to my parents - Mr Joseph Martin Podges and Mrs Joan Winnifred Podges - who have always and unconditionally supported me in all my endeavours. And secondly, to my siblings - Juan and Benji - who each in their own unique way have enriched my life and have assisted in making this journey all the more worthwhile.

I would like to acknowledge the financial support from Gibb Engineering and Architecture (Pty) Ltd that made it possible for me to study at the University of Stellenbosch.

Finally, my greatest thanks go to the Lord God Almighty for his motivation, guidance and gifts he has bestowed upon me for this study. 


\section{Abbreviations}

A-D

ASDMCon

BIM

CPD

DI

DTM

ECSA

GB

GDP

GIS

GPS

HD

HEART

IC

IDS

IMU

IP

K-S

LAN

OPI

PDF

PMBOK

QA

QC

QR

$\mathrm{R}$

RAM

$\mathrm{RE}$

RFID

$\mathrm{RH}$

SCM

$\mathrm{SU}$

UAV

UPS

VBA
Anderson-Darling

Advanced Sensor-based Defect Management on Construction Sites

Building Information Model

Continuing Professional Development

Durability Index

Digital Terrain Model

Engineering Council of South Africa

Gigabyte

Gross Domestic Product

Geographic Information System

Global Positioning System

Holding Down

Human Error Assessment and Reduction Technique

Integrated Circuit

Image Dimensioning Software

Inertial Measurement Unit

Internet Protocol

Kolmogorov-Smirnov

Local Area Network

Oxygen Permeability Index

Probability Density Function

Project Management Body of Knowledge

Quality assurance

Quality control

Quick Response

Rand

Random Access Memory

Resident Engineer

Radio Frequency Identification

Relative Humidity

Supply Chain Management

Stellenbosch University

Unmanned Aerial Vehicle

Uninterrupted Power Supply

Visual Basic for Applications 


\section{Contents}

Declaration $\quad$ i

Abstract

Opsomming iv

$\begin{array}{lll}\text { Acknowledgements } & \text { v }\end{array}$

Abbreviations $\quad$ vi

\begin{tabular}{lll}
\hline Introduction and overview & 1
\end{tabular}

1.1 Background . . . . . . . . . . . . . . . . . . . . . . 1

1.2 Problem statement . . . . . . . . . . . . . . . . . . . . . . . . . . . . . . . . . . . . . . .

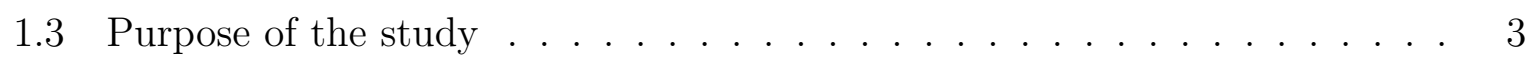

1.4 Significance of the study . . . . . . . . . . . . . . . . . . . . . . . 3

1.5 Primary research questions . . . . . . . . . . . . . . . . . . . . . . . . 3

1.6 Aims and objectives $\ldots \ldots \ldots \ldots \ldots$. . . . . . . . . . . . . . . . 4

1.7 Research design . . . . . . . . . . . . . . . . . . . . . . . . . 4

1.8 Research scope, assumptions and limitations . . . . . . . . . . . . . . . . . 4

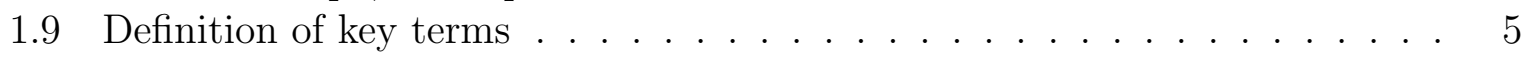

1.10 Ethical considerations . . . . . . . . . . . . . . . . . . 5

1.11 Thesis layout $\ldots \ldots \ldots \ldots \ldots \ldots$

1.12 Chapter summary . . . . . . . . . . . . . . . . . . . 6

\begin{tabular}{lll}
\hline 2 & Literature study & 7
\end{tabular}

2.1 Quality in a construction environment . . . . . . . . . . . . . . . . 8

$2.1 .1 \quad$ Construction quality $\ldots \ldots \ldots \ldots \ldots$

2.1 .2 Quality Assurance . . . . . . . . . . . . . . . . . . . . . . . . . . . . . . . . . 9

2.1 .3 Quality Control . . . . . . . . . . . . . . . . . . . . . . . . . . . . . . . 10

2.1.4 Total Quality Management . . . . . . . . . . . . . . . . . . . . . . . 10

2.1 .5 Synthesis . . . . . . . . . . . . . . . . . . . . . . . . . . . . . . . . . . . . . . 11

2.2 Quality of structural concrete . . . . . . . . . . . . . . . 11

2.2.1 Transfer mechanisms that influence concrete deterioration . . . . . 12

2.2 .2 Durability index (DI) testing as quality measure of structural concrete 13

$\begin{array}{lll}2.2 .3 & \text { Quality aspects of structural concrete to monitor during construction } 17\end{array}$

2.2 .4 Synthesis $\ldots \ldots \ldots \ldots \ldots \ldots \ldots \ldots$

2.3 Barriers preventing good concrete quality . . . . . . . . . . . . . . . . . . . 19

2.3 .1 Identified barriers in construction . . . . . . . . . . . . . . 20 
$2.3 .2 \quad$ Summary of identified barriers . . . . . . . . . . . . . . . . . 21

2.4 Result of poor construction quality . . . . . . . . . . . . . . . . . 24

2.4 .1 Rework as a result of poor quality . . . . . . . . . . . . . . 24

2.4 .2 Structural concrete construction errors that lead to rework . . . . . 25

$2.4 .3 \quad$ Summary of identified construction errors . . . . . . . . . . . . 26

2.4 .4 Synthesis . . . . . . . . . . . . . . . . . . . . 27

2.5 Quality monitoring . . . . . . . . . . . . . . . . . . . . . . . 28

2.5 .1 Quality monitoring approaches . . . . . . . . . . . . . . . 28

2.6 Technology available for quality monitoring . . . . . . . . . . . . . . . . 30

2.6 .1 Laser scanners . . . . . . . . . . . . . . . . . . . . . . . . . . . . . . . . . . . . 31

2.6 .2 Photo- and video monitoring technology . . . . . . . . . . . . . . . 34

2.6 .3 Remote identification technologies . . . . . . . . . . . . . . . . . . . 40

2.6 .4 Concrete curing sensors . . . . . . . . . . . . . . . . . . . . 43

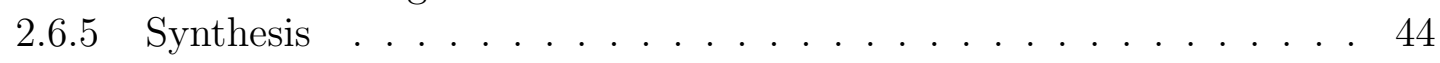

2.7 Summary of Literature study $\ldots \ldots \ldots \ldots$

\begin{tabular}{lll}
\hline & Research methodology and data collection methods & 48
\end{tabular}

3.1 Research design . . . . . . . . . . . . . . . . . . . . . . . . . . . . . . . . . .

3.1.1 Qualitative research. . . . . . . . . . . . . . . . . . . 50

3.1.2 Quantitative research . . . . . . . . . . . . . . . . 50

3.1 .3 Simulation . . . . . . . . . . . . . . . . . . . . . 50

3.2 Sample size $\ldots \ldots \ldots \ldots \ldots \ldots$. . . . . . . . . . . . . . . . . . . . . 50

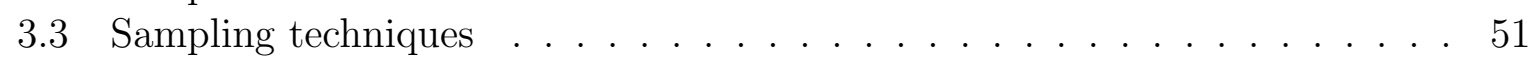

3.4 Data collection methods . . . . . . . . . . . . . . . . . . . . . 51

3.4 .1 Secondary data . . . . . . . . . . . . . . . . . . 51

3.4 .2 Primary data . . . . . . . . . . . . . . . . . . 51

3.5 Data analysis . . . . . . . . . . . . . . . . . . . . . . 54

3.5 .1 Interview data analysis . . . . . . . . . . . . . . . . . . . . 54

3.5 .2 Experimental data analysis . . . . . . . . . . . . . . . . . . 54

3.5.3 Questionnaire data analysis . . . . . . . . . . . . . . . . . 54

3.6 Chapter summary . . . . . . . . . . . . . . . . . . . . 55

\begin{tabular}{|lll}
\hline & Evaluation of technology characteristics & 57
\end{tabular}

4.1 Construction errors and preventative measures . . . . . . . . . . . . . 58

$4.1 .1 \quad$ Steel spacing $\ldots \ldots \ldots \ldots \ldots \ldots$

$4.1 .2 \quad$ Vibration and concrete compaction . . . . . . . . . . . . . . . . 59

$4.1 .3 \quad$ Grout $\operatorname{loss} \ldots \ldots \ldots \ldots \ldots \ldots$. . . . . . . . . . . . . . . . . . . 59

4.1 .4 Concrete cover . . . . . . . . . . . . . . . . . . 60

4.1 .5 Kicking of formwork . . . . . . . . . . . . . . . . . . 60

$4.1 .6 \quad$ Bleeding . . . . . . . . . . . . . . . . . . . . . . . . . 60

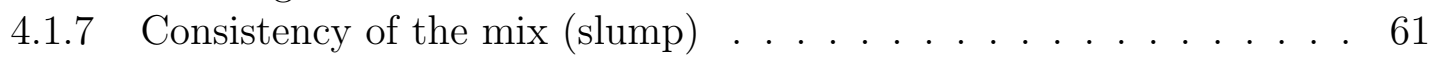

4.1 .8 Curing . . . . . . . . . . . . . . . . . . . . . . . . . 61

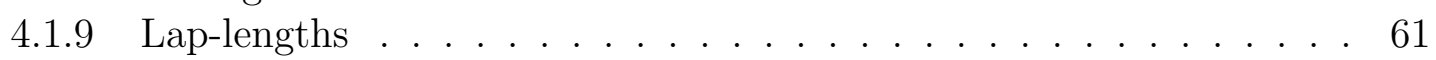

4.1 .10 Pull-out bars $\ldots \ldots \ldots \ldots$. . . . . . . . . . . . . . . . . . . . . 61

4.1 .11 Shutter boards . . . . . . . . . . . . . . . . . . 61

4.1 .12 Formwork system . . . . . . . . . . . . . . . . . . . 62

4.1 .13 Cleanliness of machinery and equipment . . . . . . . . . . . . 62 
4.2 Categorising the construction errors . . . . . . . . . . . . . . . . . . . 62

4.3 Degrees of Accuracy and tolerances . . . . . . . . . . . . . . . . . . . . 64

4.4 Evaluation of technology . . . . . . . . . . . . . . . . . . . . 65

4.4 .1 Laser scanner evaluation . . . . . . . . . . . . . . . . . . . 67

4.4 .2 Photo- and video monitoring . . . . . . . . . . . . . . . . . . 71

$4.4 .3 \quad$ Remote identification technologies . . . . . . . . . . . . . . . . . . . 80

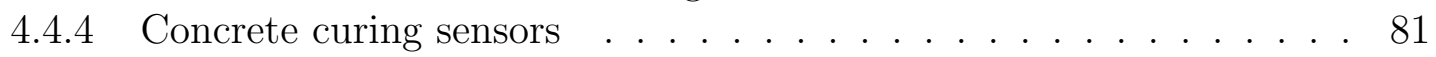

4.5 Summary of technology evaluation . . . . . . . . . . . . . . . . . . . . 81

\begin{tabular}{|lll}
5 & Impact of technology on rework & 83
\end{tabular}

5.1 Risks to mitigate through use of technology . . . . . . . . . . . . . . . 84

5.2 Effectiveness of IP cameras . . . . . . . . . . . . . . . . . . . . . . 85

5.2 .1 Using a lessons learned approach to improve quality control . . . . 86

5.2 .2 Compaction . . . . . . . . . . . . . . . . . 88

5.2 .3 Grout loss . . . . . . . . . . . . . . . . . . . . . . . . 88

5.2 .4 Kicking of formwork $\ldots \ldots \ldots$. . . . . . . . . . . . . 89

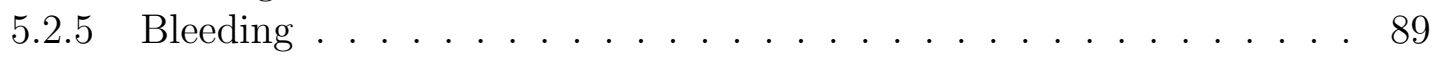

$5.2 .6 \quad$ IP camera system to monitor the identified processes . . . . . . . . 89

$5.3 \quad$ Effectiveness of IP cameras with photo-log software . . . . . . . . . . . . . 89

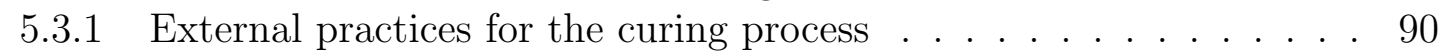

5.3 .2 Cleanliness of equipment and formwork . . . . . . . . . . . . . . 91

$5.3 .3 \quad$ Application of IP camera and field photo-log software . . . . . . . . . 91

5.4 Laser scanner and image dimensioning software for dimensioning checks . . 91

5.4 .1 Effectiveness of laser scanner to monitor steel spacing and lap-lengths 94

\begin{tabular}{|lll}
5.4 .2 & Image dimensioning software to monitor steel spacing and lap-lengths 95
\end{tabular}

5.4 .3 Laser scanner to monitor concrete cover . . . . . . . . . . . . . . . 97

5.4 .4 Laser scanner to monitor pull-out bars and HD bolts . . . . . . . . . 98

5.4 .5 Image dimensioning software with photo-log software to monitor concrete mix consistency . . . . . . . . . . . . . . . . . 98

5.5 Laser scanner for applications other than dimensioning . . . . . . . . . . . 100

5.5 .1 Identification of formwork and shuttering condition . . . . . . . . . 101

5.5.2 Dimensions and alignment of formwork . . . . . . . . . . . . . 103

5.6 Quick response code and radio frequency identification effectiveness . . . . 104

5.6 .1 Material handling . . . . . . . . . . . . . . . . . 105

5.7 Concrete curing sensor effectiveness . . . . . . . . . . . . . . . 107

5.7 .1 Moisture condition during curing . . . . . . . . . . . . . . . 108

5.8 Summary of technology effectiveness . . . . . . . . . . . . . . . . 109

6 Questionnaire Survey 111

6.1 Participant information . . . . . . . . . . . . . . . . . . . . . 112

6.2 Questionnaire results . . . . . . . . . . . . . . . . . . . . . . . . . . 113

6.3 Synthesis . . . . . . . . . . . . . . . . . . . . . . . . . . . 119

7 Decision model logic 120

7.1 Model overview . . . . . . . . . . . . . . . . . . . . . . . . . . . . . . . 121

7.2 Step 1: Attribute ratings . . . . . . . . . . . . . . . . . . . . . . 121

7.3 Step 2: Category scores . . . . . . . . . . . . . . . . . . . . . . 125 
7.4 Step 3: Risk magnitude. . . . . . . . . . . . . . . . . . . . . . . . 127

7.4 .1 Likelihood criteria . . . . . . . . . . . . . . . . . . . . . . . . . 129

7.4 .2 Impact criteria $\ldots \ldots \ldots$. . . . . . . . . . . . . . . 130

7.4 .3 Risk magnitude calculation . . . . . . . . . . . . . . . . . . . 131

7.5 Step 4: Risk mitigation . . . . . . . . . . . . . . . . . . . . . 133

7.6 Step 5: Investment suggestion . . . . . . . . . . . . . . . . . . . . . 135

8 Model validation $\quad 144$

8.1 Scenario analysis . . . . . . . . . . . . . . . . . . . . . 145

8.1.1 Scenario 1: Poor performance expectancy from all categories . . . . 145

8.1.2 $\quad$ Scenario 2: Fairly good performance from management . . . . . . . 153

8.1.3 Scenario 3: Fairly good performance from labour and management. 155

8.1.4 Scenario 4: Fairly good performance from labour, management and

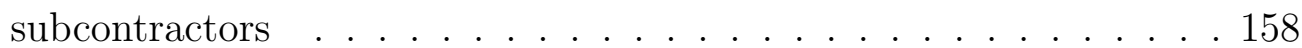

8.1.5 Scenario 5: Good performance from all categories and very good management performance . . . . . . . . . . . . . 161

8.1 .6 Summary of various scenarios . . . . . . . . . . . . . . . . . . 162

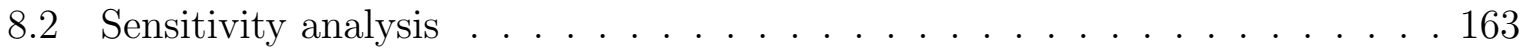

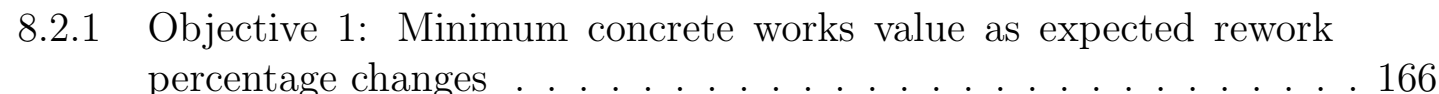

8.2 .2 Objectives 2 and 3: Variability of net rework savings . . . . . . . . 168

8.3 Validation interviews $\ldots \ldots \ldots \ldots$. . . . . . . . . . . . . . 172

8.3 .1 Summary of interviews . . . . . . . . . . . . . . . 173

$\begin{array}{llr}9 & \text { Conclusions and recommendations } & 175\end{array}$

9.1 Summary . . . . . . . . . . . . . . . . . . . . . . . . 175

9.1.1 Aims and objectives . . . . . . . . . . . . . . 176

9.2 Conclusions . . . . . . . . . . . . . . . . . . . . . . 177

9.2 .1 Results conclusion . . . . . . . . . . . . . . . . . 177

9.3 Recommendations . . . . . . . . . . . . . . . . . . . . . . . . . . . . . . . . . . . 179

9.3 .1 Decision model . . . . . . . . . . . . . . . . . . . . . . . 179

9.4 Research limitations . . . . . . . . . . . . . . . . . . . . . . . . . . . . . 180

\begin{tabular}{lr}
\hline Appendices & 189
\end{tabular}

A $\quad$ The importance of curing. . . . . . . . . . . . . . . . . . . . . . . 190

B Evaluation forms for technologies . . . . . . . . . . . . . . . . . . . . 191

C $\quad$ Probability density functions . . . . . . . . . . . . . . . . . . . . 197

C.1 Approach for the laser scanner . . . . . . . . . . . . . . . . . . . . 197

C.2 Approach for IDS effectiveness . . . . . . . . . . . . . . . . . . . . . 198

D Technological specifications and cost . . . . . . . . . . . . . . . . 200

D.1 Laser scanning technology . . . . . . . . . . . . . . . . . . . 200

D.2 Summary of the cost for the laser scanner systems . . . . . . . . . . 203

E $\quad$ Photography and video monitoring . . . . . . . . . . . . . . . . 204

E.1 Software application for photo-log daily reporting . . . . . . . . . . 205

E.2 Mobile software that is used to measure dimensions on images . . . 205

E.3 $\quad$ Internet Protocol Cameras . . . . . . . . . . . . . . . . . . 205

F $\quad$ Radio Frequency Identification tags (RFID) . . . . . . . . . . . . . 206 
F.1 Technical requirements and cost of system . . . . . . . . . . . 207

F.2 Quick response (QR) codes . . . . . . . . . . . . . . . . 207

G Concrete curing sensors . . . . . . . . . . . . . . . . . . . . . 208

H Summary of cost for the various systems . . . . . . . . . . . . . . . 208

I Survey . . . . . . . . . . . . . . . . . . . . . . . . . . 209

J Validation interviews . . . . . . . . . . . . . . . . . . . . 221 


\section{List of Figures}

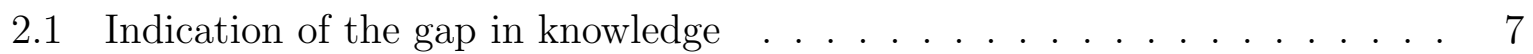

2.2 Summary of Section $\mid 2.1 \quad \ldots \ldots \ldots \ldots$

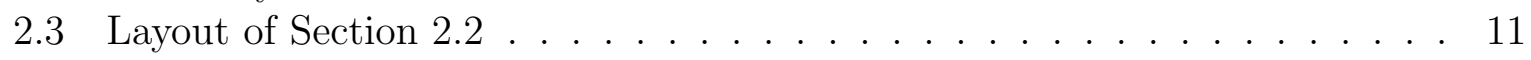

2.4 Increase in corrosion levels over time (Muigai et al., 2012)] . . . . . . . . . . 14

2.5 Carbonation depth over time for three different OPI values(Mackechnie and Alexander, 2002)] . . . . . . . . . . . . . . . 15

$2.6 \quad$ Probability of failure for a given cover depth (Muigai et al. [2012)] . . . . . 15

2.7 Corrosion rate for a given cover $(\operatorname{Scott}[2004)] \ldots \ldots$. . . . . . . . 16

2.8 Summary of quality barriers that are discussed in Section 2.3 . . . . . . . . 23

2.9 Summary of quality checks to ensure good quality concrete . . . . . . . . . 27

2.10 Using a laser scanner to produce a 3D-model of a construction site (Cheng and Teizer, 2013). . . . . . . . . . . . . . . . . . 34

2.11 Process followed to generate point cloud models from images (Harwin and

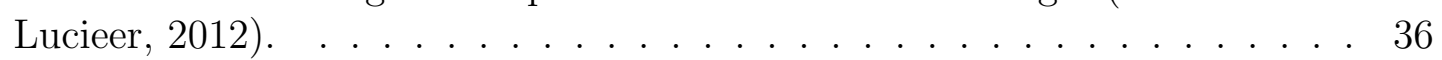

2.12 Wireless sensors that are embedded in concrete (Structural Health Systems,

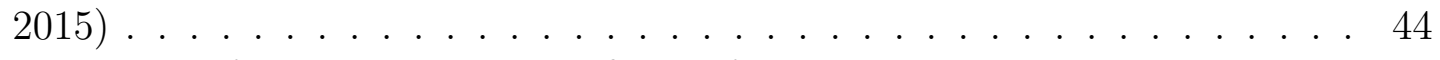

2.13 Indication of the gap in primary fields of research pertaining to the study. $\quad 46$

3.1 Layout of decision model and data required to populate the model . . . . . 49

3.2 Overview of the following Chapters . . . . . . . . . . 56

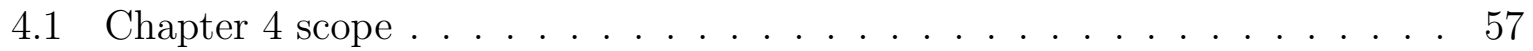

4.2 Content presented in Chapter $4 \ldots \ldots \ldots$. . . . . . . . . . . 58

4.3 Summary of identified construction errors sub-divided under main categories of influence . . . . . . . . . . . . . . . 64

4.4 Meshed model after using a laser scanner to scan a reinforcement beam-cage 68

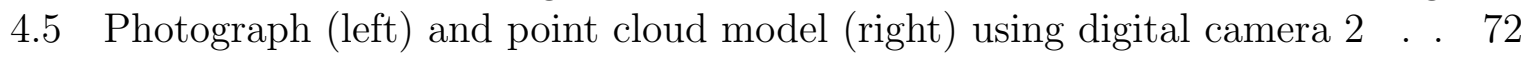

4.6 Shaded surface (left) and textured model (right) using digital camera 2 . . 73

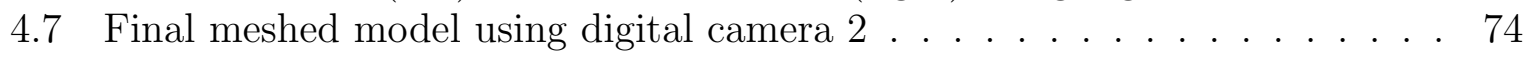

4.8 Point cloud model generated with software that requires less computation

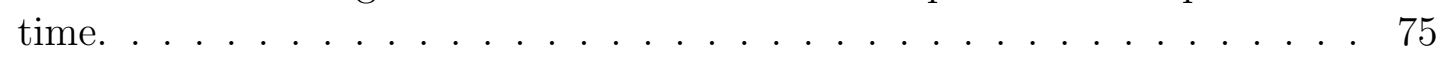

4.9 Locations measured on the reinforced beam-cage . . . . . . . . . . . . . 76

4.10 Illustration (in the vertical plane) of the vertical angle from which photos 7

4.11 Illustration (horizontal plane) of the horizontal angle from which photos were taken. . . . . . . . . . . . . . . . . 77

5.1 Scope of Chapter $5 \ldots \ldots \ldots \ldots \ldots$ 
5.2 Structure of content in Chapter 5 . . . . . . . . . . . . . . . . . . 84

5.3 Process of deriving effectiveness of IP camera $\ldots \ldots \ldots$. . . . . . . . . . 86

5.4 Content of this Section relative to other Sections . . . . . . . . . . . . . . 90

5.5 Content of this Section relative to other Sections in this Chapter . . . . . . 92

5.6 Histogram for laser scanner capability to monitor steel spacing and lap-

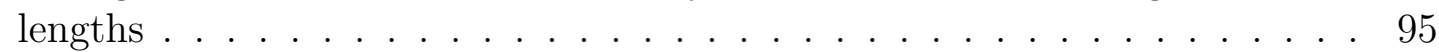

5.7 Histogram for IDS capability to monitor steel spacing and lap-lengths . . . 96

$5.8 \quad$ Histogram for laser scanner capability to monitor concrete cover $\ldots$. . . . . 97

5.9 Histogram for the laser scanner system's effectiveness to monitor the pullout bars and HD bolts . . . . . . . . . . . . . . . . . . . . . 98

5.10 Histogram for IDS capability to monitor concrete consistency . . . . . . . . 100

5.11 Content of this section relative to other Sections in this Chapter . . . . . . 101

5.12 Box plot, as determined by the author, to identify outliers and then calculate technology effectiveness . . . . . . . . . . . . . . . 103

5.13 Content of this Section relative to content of other Sections in this Chapter 105 5.14 Content of this Section relative to content of other Sections in this Chapter 108

6.1 Scope of Chapter $6 \ldots \ldots \ldots \ldots$. . . . . . . . . . . . . . . . . . . . . . .

7.1 Scope of Chapter 7 . . . . . . . . . . . . . . . . . . 120

7.2 Overview of Step 1 in the decision model . . . . . . . . . . . . . . . . . . . 122

7.3 Step 1 model logic $\ldots \ldots \ldots$. . . . . . . . . . . . . . . . . . . 123

7.4 Graphical user interface with expected performance from labour as user

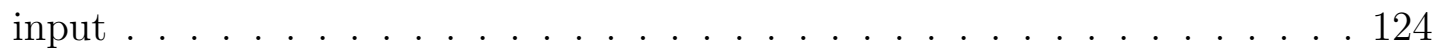

7.5 Overview of Step 2 in the decision model . . . . . . . . . . . . . . . . 125

7.6 Step 2 model logic $\ldots \ldots \ldots$. . . . . . . . . . . . . . . . 126

7.7 Overview of Step 3 in the decision model . . . . . . . . . . . . . . . . 128

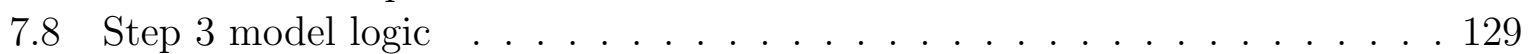

7.9 Overview of Step 4 in the decision model . . . . . . . . . . . . . . . . . . . 133

7.10 Step 4 model logic . . . . . . . . . . . . . . . . . . . . . . . . . . . . . . . 134

7.11 Overview of Step 5 in the decision model . . . . . . . . . . . . . . . 135

7.12 Step 5 model logic . . . . . . . . . . . . . . . . . . . . . 136

8.1 Scope of Chapter 8 . . . . . . . . . . . . . . . . . . . . . . 144

8.2 Labour attribute ratings for scenario 1 . . . . . . . . . . . . . . . . . 146

8.3 Management attribute ratings for scenario 1 . . . . . . . . . . . . . . . 147

8.4 Subcontractor attribute ratings for scenario $1 \ldots$. . . . . . . . . . . . 148

$8.5 \quad$ Plant and equipment attribute ratings for scenario 1 . . . . . . . . . . . . 149

8.6 Risk magnitudes identified and user chooses which risks to mitigate . . . . 150

8.7 Cost allocation of rework expected from each risk that requires mitigation. 151

8.8 Rework savings expected from each technological system for scenario 1 . . 152

8.9 Risk magnitudes and classification for scenario 2 . . . . . . . . . . . . . . . 154

8.10 Risk magnitudes and classification for scenario 3 . . . . . . . . . . . . . . 157

8.11 Risk magnitudes and classification for scenario 4 . . . . . . . . . . . . . . . 160

8.12 Risk magnitudes and classification for scenario 5 . . . . . . . . . . . . . . . 162

8.13 Reduction in rework for scenarios 1 to 5 . . . . . . . . . . . . . . 163

8.14 Brief outline of sensitivity analyses objectives . . . . . . . . . . . . . . . 165

8.15 Sensitive zone for analyses that were conducted . . . . . . . . . . . . . . 168 
8.16 Graph showing expected rework reduction versus estimated rework values . 169

8.17 Rework savings versus concrete works value . . . . . . . . . . . . . 170

8.18 Expected savings by mitigating rework versus estimated rework values. . . 171

$1 \quad$ Graph showing the compressive strength of concrete over a given time period 190

2 Graph showing the carbonation rate as the relative humidity changes . . . 191

$3 \quad$ PDF, with mean of $0.865 \%$ and variance of 0.387 , for laser scanner software monitor steel spacing . . . . . . . . . . . . . . . . . 198

4 Log-normal PDF with mean of $0.923 \%$ and variance of 1.214 , for laser scanner software monitor steel spacing . . . . . . . . . . . . . . 200

5 System 1: Can be used to generate scans while walking, flying and driving (3D Laser mapping, 2016)] . . . . . . . . . . . . . . . . . . 201

6 System 2: Handheld scanner that shows scanned objects while scanning

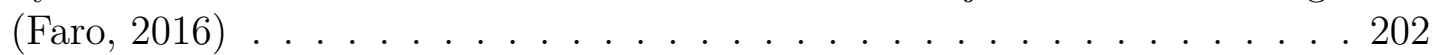

7 System 3: Tripod scanner (Faro 2016$)$. . . . . . . . . . . . . . . . . 202

$8 \quad$ IP camera network setup . . . . . . . . . . . . . . . . . . . 206

9 $\quad$ RFID system $($ Ropraz $[2008)] \ldots$. . . . . . . . . . . . . . . 207

10 Concrete sensors that work with probes (The Transtec Group $[2015$ )] . . . . 208

11 More questions from survey $\ldots \ldots$. . . . . . . . . . . . . 220 


\section{List of Tables}

2.1 Quality monitoring systems identified by $[\operatorname{Smallwood}[(2000)] \ldots$. . . . . . . 28

$4.1 \quad$ Experience of interview participants . . . . . . . . . . . . . . . . . . . . . 59

4.2 Accuracy requirements according to SANS 2001-CC1 (2007). . . . . . . . . 65

4.3 Evaluation criteria for technological systems $\ldots \ldots$. . . . . . . . . 66

4.4 Accuracy deviations determined from laser demonstration data . . . . . . . 68

$4.5 \quad$ Evaluation criteria used to assess laser scanner . . . . . . . . . . . . . . . . 69

4.6 Evaluation of the laser scanner after conducting scans in the laboratory . . 70

4.7 Technical specifications of the cameras used in the experiment. . . . . . . . 72

4.8 Dimensions measured when generating a point cloud model from camera 2 images. . . . . . . . . . . . . . . . . . . 73

4.9 Evaluation of the pointcloud software after tests in the laboratory . . . . . 74

4.10 Test results for the image dimensioning software through use of a mobile

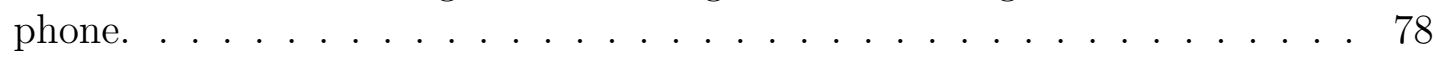

4.11 Evaluation of the software that is used to take measurements on photos. $\quad 79$

5.1 Requirements of technology to assist in a lessons learned approach . . . . . 88

5.2 Technology attributes requirements to assist in a lessons learned approach 90

5.3 Rating criteria defined for the monitoring of steel spacing and lap-lengths . 93

5.4 Rating criteria defined for the monitoring of concrete cover, pull-out bars and position of HD bolts . . . . . . . . . . . . . . . 93

5.5 Experimental values obtained from tests in the laboratory . . . . . . . . . 96

5.6 Rating criteria defined for the monitoring of concrete consistency . . . . . 99

5.7 Rating criteria defined for the monitoring of shutter surface deformations . 102

5.8 Rating criteria defined for the monitoring of dimensions and alignment of

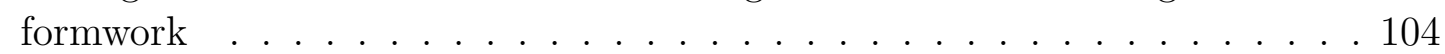

5.9 Factors that increase unreliability and the proportion of the effect of each . 106

5.10 Factors that increase unreliability and the proportion of the effect of each .107

5.11 Summary of the effectiveness determined for each of the technological solutions to monitor the identified construction errors that could occur . . . 109

6.1 Participant information $\ldots \ldots \ldots \ldots$. . . . . . . . . . . . . . . . . 112

6.2 Results from Question 1 . . . . . . . . . . . . . . . . . . . . . . . . . . . . 114

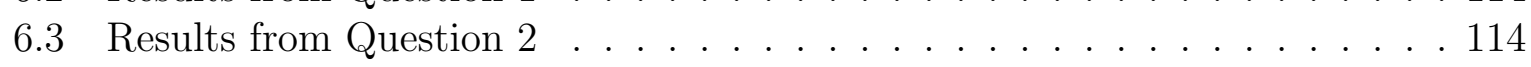

6.4 Results from Question 3 . . . . . . . . . . . . . . . . . . . . . . . 115

6.5 Results from Question 4 . . . . . . . . . . . . . . . . . . . . . . . 115

6.6 Risk impact criteria in numerical values . . . . . . . . . . . . . . . . . . . . 116

6.7 Results from Question $9 \ldots \ldots$. . . . . . . . . . . . . . 117 
6.8 Results from Question 10 . . . . . . . . . . . . . . . . . . . . . . 117

6.9 Results from Question 11 . . . . . . . . . . . . . . . . . . . . . . . . . 118

6.10 Results from Question 12 . . . . . . . . . . . . . . . . . . . . . 118

6.11 Expected rework cost on the structural concrete works . . . . . . . . . . . 119

7.1 Overview of the risk management model . . . . . . . . . . . . . . . . . . . 121

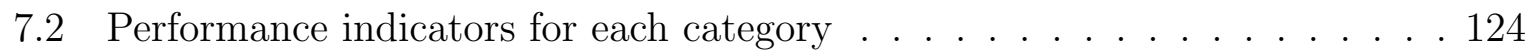

7.3 Example of Step 2 in the model for the labour category . . . . . . . . . . . . 127

7.4 Risk likelihood criteria . . . . . . . . . . . . . . . . . . . . 130

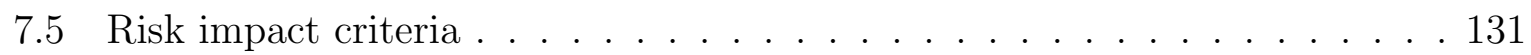

7.6 Risk matrix used to determine risk magnitude . . . . . . . . . . . . . . . . 131

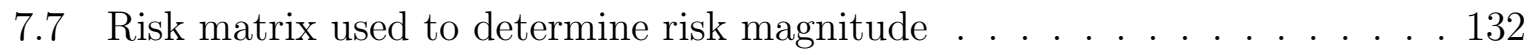

7.8 Risk matrix underlying magnitude values . . . . . . . . . . . . . . . . . . . 132

7.9 Construction errors and technological mitigation solutions for the identified

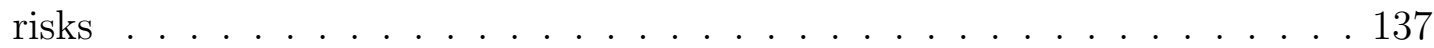

\begin{tabular}{ll|l|l|l|}
\hline 7.10 & Example continued from Table & where user provides expected rework
\end{tabular}

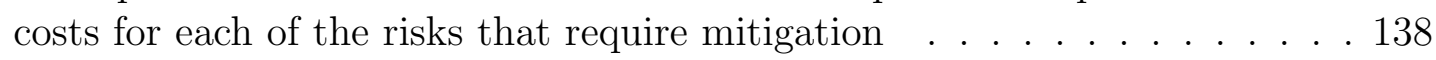

7.11 Example where the allocated\% of rework costs are adjusted to include effects of the risk magnitudes . . . . . . . . . . . . . . . . . . 140

7.12 Potential rework saved by using IP camera . . . . . . . . . . . . . . . . . 141

7.13 Potential rework saved by using IDS in conjunction with logging software . 141

7.14 Potential rework saved by using laser scanner . . . . . . . . . . . . . . 142

7.15 Potential rework saved by using concrete sensors . . . . . . . . . . . . . . . 142

7.16 Total net savings from combination of technologies . . . . . . . . . . . 143

8.1 Technology used to mitigate each risk and rework saved from mitigating each risk . . . . . . . . . . . . . . . . . . 152

8.2 Performance indicators for each category in scenario 2 . . . . . . . . . . . . 153

8.3 Net rework reduction by each technological system for scenario 2 . . . . . . 155

8.4 Performance indicators for each category in scenario 3 . . . . . . . . . . . 156

8.5 Net rework reduction by each technological system for scenario 3 . . . . . . 158

8.6 Attribute ratings for each category in scenario 4 . . . . . . . . . . . . . 159

8.7 Net rework reduction by each technological system for scenario 4 . . . . . . 160

8.8 Attribute ratings for each category in scenario 5 . . . . . . . . . . . . . 161

8.9 Concrete works value required for technological investment to be feasible . 167

8.10 Net rework savings (losses) for various combinations of project value and expected rework percentages . . . . . . . . . . . . . . . 171

8.11 Experience of the participants in the validation interviews . . . . . . . . . 172

8.12 Validation questions answered by participants . . . . . . . . . . . . 173

$1 \quad$ Evaluation criterion used to assess the capability of a technological system 191

2 Evaluation criterion used to assess the capability of image dimensioning

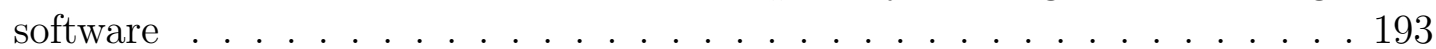

3 Evaluation criterion used to assess the capability of the internet protocol

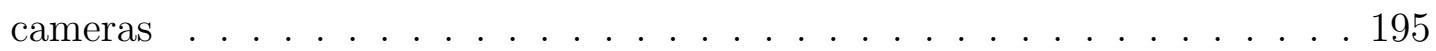

$4 \quad$ P-values obtained for the K-S and A-D goodness-of-fit tests. . . . . . . . . 197

$5 \quad$ P-values obtained for the K-S and A-D goodness-of-fit tests. . . . . . . . . 199

$6 \quad$ Summary of the costs for the various systems identified . . . . . . . . . . . 204 
$7 \quad$ Technical specifications of a proposed IP camera . . . . . . . . . . . . . . 205

8 Summary of the cost for the various technological systems identified . . . . 209

9 $\quad$ Opinions obtained from participant 1 . . . . . . . . . . . . . . . . . 221

10 Opinions obtained from participant 2 . . . . . . . . . . . . . . . . . . 222

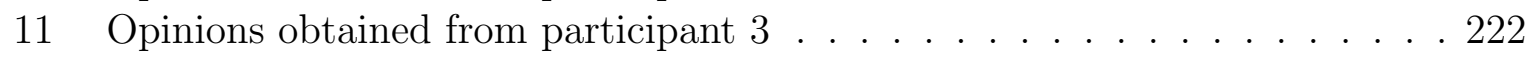

12 Opinions obtained from participant 4 . . . . . . . . . . . . . . 223

13 Opinions obtained from participant 5 . . . . . . . . . . . . . . . 223 


\section{Chapter 1}

\section{Introduction and overview}

This study investigates a method to choose technology that improves the quality control of structural concrete elements, such as beams, columns, slabs and reinforced concrete walls. The construction quality of structural concrete is often poor and leads to excessive rework. Various technology systems were investigated and a decision model was developed to assist a contractor in the investment of funds toward the acquisition of technology.

\section{$1.1 \quad$ Background}

Transfer mechanisms, such as diffusion, absorption and permeability, lead to concrete deterioration. These mechanisms are catalysed by the poor construction of structural concrete (Basheer, Kropp and Cleland, 2001). Various barriers were identified that prevent good quality of structural concrete. Smallwood (2000) identifies these barriers as poor site management, lack of contractor experience and inadequate skills and training. The CIDB: South Africa (2016) identified barriers similarly to that of Smallwood (2000). Jergeas and Ruwanpura (2010) identified that projects in construction are fast-tracked to make up for lost time which then leads to construction errors.

The result of poor construction quality is rework. Rework exists due to non-conformance, quality deviations and failure to conform to quality requirements (Zhang, Haas, Goodrum, Caldas and Granger, 2012). In a study conducted by Shammas-Toma et al. (1996), in the United Kingdom, it was identified that corrosion was resulting in the need for millions of pounds worth of remedial work to concrete bridges. It was suggested that future expenditure of this nature could be reduced by improving the quality of workmanship. A study by Farrington and Ledbetter (1992) analysed the causes of deviations in construction quality. They found that in $15.5 \%$ of the projects studied, deviations were caused by errors during construction. Construction deviations made up 2.5\% of the total project cost. However, Harvey and Kagan (1990) found a much higher cost of rework at $15 \%$ of the total project cost. Moreover, Harvey and Kagan (1990) said that the secondary impact of quality deviations could be worse. Smallwood (2000) found that rework constitutes $6 \%$ of total project cost for CIDB 5-9 contractors. In a study conducted by Harvey and Kagan (1990), panellists consisting of owners, contractors and designers were asked for their opinion on the need for quality control. The panellists agreed that quality control is essential in construction and it is worth the costs to implement. Furthermore, the approaches used for quality monitoring and control on construction sites are not effective enough to identify 
defects early in the construction process. From the survey in this study it was found that in South Africa, construction rework consists $4-6 \%$ of the cost of concrete works when building structures that primarily consist of structural elements.

It was reasoned that by improving the quality control during construction, the quality of structural concrete is also improved. Smallwood (2000) identified quality monitoring systems and ranked them according to the systems best rated by clients, designers and project managers. The quality monitoring system consisting of inspections and visual checks was rated the best. This provides the opportunity of using technology to assist in the quality monitoring consisting of inspections and visual checks.

Various systems such as laser scanners, internet protocol cameras, radio frequency identification tags and concrete sensors have previously been used by authors to test the use thereof in construction. Laser scanners have been used in surveying to create topographical maps and also for progress monitoring. Cameras have been used to generate point cloud models of the works and for inspection through use of an unmanned aerial vehicle. Remote identification technologies have been used in supply chain management and concrete cube testing laboratories. Concrete sensors have been used during curing to monitor the moisture condition of the concrete. These technologies have been used in construction, but not specifically to improve the quality control of structural concrete during construction.

This study investigates the effectiveness of these technologies for quality control during concrete construction and assists the user in the investment decision making process to acquire these technologies. The scope is limited to the quality control of structural concrete elements such as beams, columns, slabs and walls since surveys in this study showed that the highest rework cost occurs for these types of concrete construction works. The surveys compared the rework of large structural footings and structural elements. Water retaining structures and gravity structures were not considered for this study.

The effectiveness of the technologies were evaluated for the construction errors that are probable to lead to rework. The various barriers that lead to poor quality were identified as attributes of labour, management, subcontractors, and plant and equipment. To quantify the effect of these attributes on concrete quality, questionnaires were distributed to industry experts. Furthermore, the questionnaires obtained data of the influence that each category (labour, management, subcontractors, and plant and equipment) have on the expected construction errors. This data was useful to determine the risk magnitude of each of the construction errors that could lead to rework of structural concrete. The model suggests an investment decision based on the risk and expected rework cost on a project.

\subsection{Problem statement}

There is a concern regarding the cost of rework on projects in South Africa that primarily consist of structural concrete elements. Research identified the causes of rework, but little research was found on the mitigation of these errors that eventually lead to rework. The 
gap in knowledge exists where a lack of methods were identified to improve the quality control during construction. Moreover, little research is available on the use of technology to improve the quality control of structural concrete.

\subsection{Purpose of the study}

The purposes of this study is to identify the construction errors that lead to rework and to investigate the technologies available to improve the quality control of structural concrete. This information is used to develop a decision model that assists the contractor in the investment decision of these technologies. It is reasoned that technology continually improves; therefore, the emphasis of the study is on the development of a decision model and the logic in the model used to choose technology for a project. Furthermore, current technologies are investigated, but the determination of their effectiveness to prevent rework was not intensively investigated.

The study makes use of qualitative methods to obtain information of current construction errors that lead to rework and preventative measures to reduce rework. Moreover, quantitative methods are used to analyse risk with data determined from experts in the construction industry. The risks are used in the decision model to determine which construction errors require mitigation.

\subsection{Significance of the study}

The significance of the study lies in the improvement of construction quality and reduction of rework. The client receives an improved product that is more durable. Furthermore, the project profits increase by reducing rework.

Structures that have poor durability could potentially lead to structural failure. Moreover, loss of life and significant damage. The author also aims to create a pro-technology attitude towards the contractor since the construction industry is reluctant to change and is still committed to traditional methods of building (Identified from the interviews in Chapter 8).

\subsection{Primary research questions}

The following research questions were identified:

1. What is the risk magnitude of the primary causes of rework during structural concrete construction?

2. Can the currently available technologies be used to reduce the rework on structural concrete?

3. Can a decision model be developed that assists a contractor in the investment decision making of technology? 


\subsection{Aims and objectives}

The aim of this study is to develop a decision model, based on risk and cost, to assist a contractor in the investment decision of acquiring technology to reduce rework on concrete.

To achieve the aim of this study, the following objectives are defined:

1. Interview industry experts to identify causes of concrete rework during construction

2. Interview industry experts to identify mitigation measures to prevent rework during construction

3. Evaluate available technologies' capability and effectiveness to improve quality control through implementation of mitigation measures

4. Quantify the risk magnitude of construction errors through questionnaire data.

\subsection{Research design}

For this study a mixed-method design approach is used to develop a decision model. Qualitative and quantitative research methods are used in the development of the model. Survey based, experimental based and simulation based research methods are used.

Interviews were conducted with industry experts to identify the critical construction errors that lead to rework and how to prevent these errors to avoid rework. The interviews contained more than 10 open-ended questions and each took more or less 90 minutes to complete.

Questionnaires were used to quantify the likelihood and impact of risks that lead to rework. The questionnaire was developed with the assistance of the statistics department of Stellenbosch University (Dr D. Nel).

Simulations are used to attempt to represent practical situations or processes mathematically using appropriate key variables.

\subsection{Research scope, assumptions and limitations}

In this study the current uses of technology in construction is identified. Moreover, other technology systems are identified that can be used in construction to improve quality control. These technologies are assessed for its effectiveness to reduce concrete rework and are used in the decision model as risk mitigating solutions. The research scope is narrowed by focussing only on concrete rework where structural elements, such as beams, columns, slabs and reinforced walls are used. Moreover, the study focuses on rework as a result of erroneous construction activities during the execution of the concrete works rather than errors during the design phase or caused by incorrect specifications. Survey data was obtained from experts in the Western-Cape, South Africa. The questionnaires included 41 participants and the interviews consisted of 9 participants. 
It is assumed that the data found in this study is a representation for the whole of South Africa. It is not generalizable for other countries due to variations in education level and building conditions. It is assumed that all the participants of the surveys answered truthfully and accurately based on their personal experience.

This study is subject to various limitations. It is assumed that the proposed mitigation measures that are identified are adequate to prevent construction errors. The actual accuracy of the decision model could vary on real-life projects. The researcher was limited by time and could not test the model on real-life construction projects to determine its accuracy. Furthermore, the researcher was limited by a lack of funds to acquire all the technologies for testing. Therefore, some of the technologies were not evaluated through practical examples on site or in the laboratory.

\subsection{Definition of key terms}

Construction activities: Construction activities refer to the processes engaged in to construct structural elements.

Construction errors: Construction errors refer to errors that occur during construction activities.

Rework: The unnecessary effort of redoing an activity because it was inaccurately done the first time (Love and Li, 2000).

Risks: Potential construction errors that lead to rework.

Decision model: A computer based system that predicts the outcomes of decisions.

\subsection{Ethical considerations}

In order to comply with internationally accepted ethical standards, the name of participant organisations and individuals will not be recorded on research instruments. No compensation will be paid to any respondent or participant in the study. An ethical application procedure was filed and accepted by the University of Stellenbosch (SU-HSD003014).

\subsection{Thesis layout}

Chapter 1: Introduction and overview - the introductory chapter comprises of the study background, problem statement, purpose, significance, research questions, aims and objectives, research design, scope, assumption, limitations, key terms and thesis layout.

Chapter 2: Literature study - the literature comprises research on quality in construction, quality of structural concrete, barriers that prevent good quality, construction 
errors, quality monitoring and technology available for quality monitoring.

Chapter 3; Research methodology and data collection methods - the research methodology provides information on the research design, data collection methods and data analysis.

Chapter 4: Evaluation of technology characteristics - the technology assessment first identifies construction errors to monitor and then sub-divides these errors under categories that cause the error. The various technology systems are then evaluated to determine which construction errors it could monitor to improve quality control.

Chapter 5: Impact of technology on rework- the effectiveness of the technological systems, to monitor the construction activities in such a manner that construction errors are prevented, were determined in this Chapter.

Chapter 6: Questionnaire Survey - questionnaires were distributed to industry experts and the data was used to quantify the risk magnitudes in the decision model.

Chapter 7; Decision model logic - the decision model is developed and explained by means of examples.

Chapter 8: Model validation - the decision model is validated through: a fictitious case study with various scenarios; sensitivity analyses and interviews with industry experts.

Chapter 9: Conclusions and recommendations- the author makes conclusions and recommendations regarding the study that was conducted.

\subsection{Chapter summary}

This Chapter showed the negative influence of poor quality on projects. Poor quality leads to rework, which is undesirable since it could cost between $4-6 \%$ of the total project cost. The significance of this study lies in the savings that the contractor can increase by reducing rework. Furthermore, the quality of structures are improved.

The gap in knowledge was identified where there is a lack of research on using technology as quality control measures during the construction of structural concrete. Furthermore, no formal method of quantification was found to assist the contractor in the acquisition of technology to reduce concrete rework. The aim of this study is to develop a decision model that will assist the contractor in an investment decision for acquiring technology.

To reach the aim of the study it is required that sound instruments be used to collect data and the instruments be used in the correct manner. The key data that needs to be collected is the data that is used to predict the outcome of the decision model. This would be the important construction errors that are identified as risks and the impact of labour, management, subcontractors, and plant and equipment on these errors. 


\section{Chapter 2}

\section{Literature study}

To achieve the aim of this study (decision model for technology investment to reduce rework), information is required regarding construction quality, common errors that occur and barriers that prevent good quality. Furthermore, the available technologies should be investigated to determine how applicable it is to use as quality control method. The literature study assists in identifying a gap in research where technology is used to reduce concrete rework.

Figure 2.1 illustrates the gap in knowledge that is addressed by this study. Barriers in construction prevent good quality concrete. Because of construction barriers, increased quality control is required to not compromise the quality of construction. Where a lack of quality control is present, construction errors and rework is the result. Technology solutions are proposed to improve the quality control of construction.

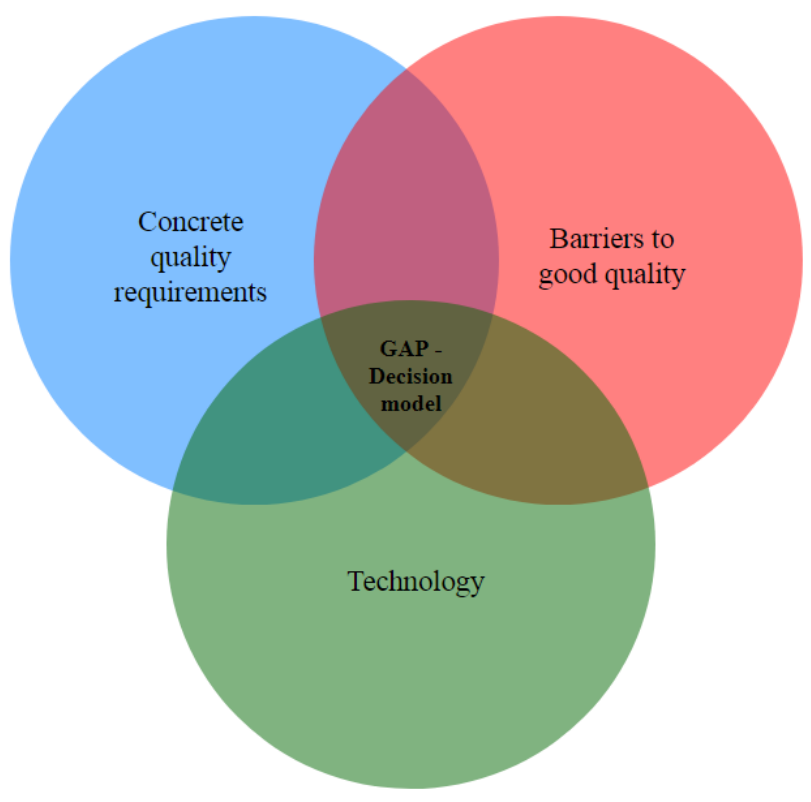

Figure 2.1: Indication of the gap in knowledge

The topics in this Chapter aim to identify quality requirements during construction, barriers toward obtaining good quality and technologies available for quality control. The 
following Chapters (after literature) aim to fill the identified gap. The author of this study aims to fill the gap by means of a decision model that considers quality requirements and project barriers to provide a recommendation on technology that can be used to reduce rework on concrete.

In Section 2.1, the term "Quality" is discussed and how it is relevant in a construction environment. In a construction environment it is important to understand the difference between construction quality, quality assurance, quality control and a total quality management system.

In Section 2.2, the micro-level factors that cause poor quality of structural concrete are considered.

In Section 2.3, the barriers toward obtaining good concrete quality are discussed.

In Section 2.4, the result of poor quality is discussed. Furthermore, the construction errors that occur during the construction of structural concrete is identified from a study by Solomons (2014).

In Section 2.5, various approaches toward quality monitoring are discussed.

In Section 2.6, various technological systems that were used in previous construction projects are identified and discussed. Other technological systems that could be used to improve construction quality are also identified.

\subsection{Quality in a construction environment}

Section 2.1 provides an overview of construction quality, quality assurance, quality control and total quality management. Figure 2.2 shows a summary of the Section to follow.

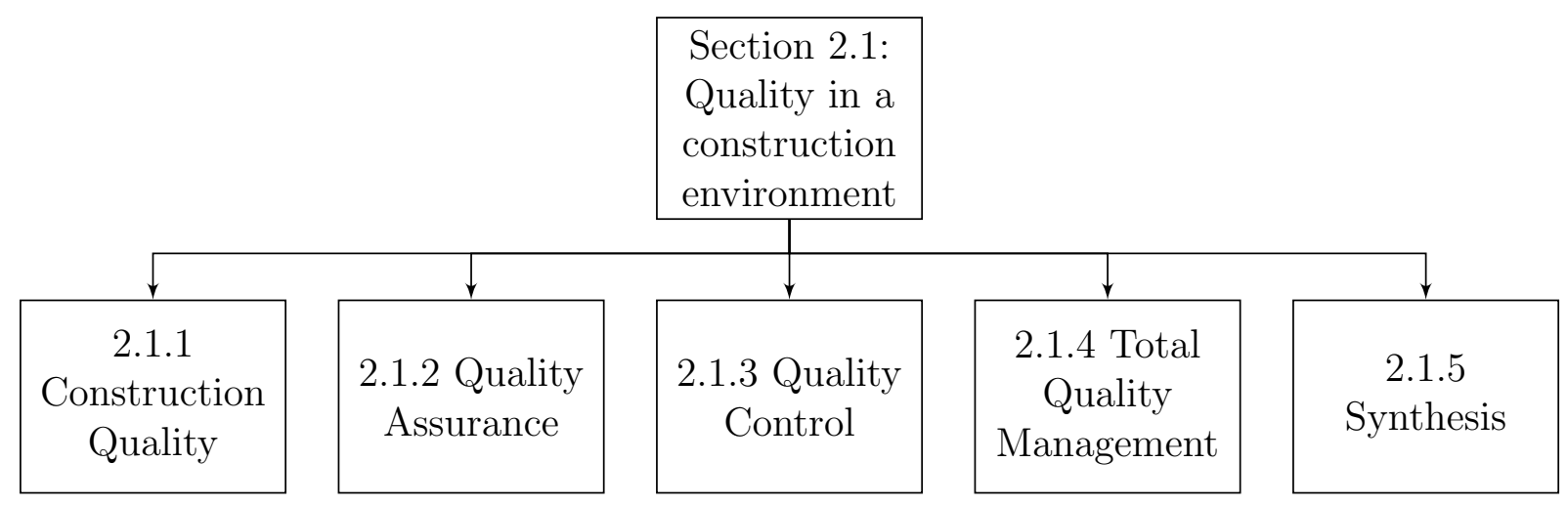

Figure 2.2: Summary of Section 2.1

\subsubsection{Construction quality}

The Business Dictionary (2013) defines Quality as: 
"A measure of excellence or state being free from defects, deficiencies and significant variations. It is brought about by strict and consistent commitment to certain standards that achieve uniformity of a product in order to satisfy specific customer or user requirements."

The ISO 9000 (1994) defines quality as the degree to which a set of inherent characteristics fulfils requirements. Duncan, Thorpe and Sunmer (1990) state that quality refers to standards and the ways and means by which those standards are achieved, maintained and improved upon. However, Harris and McCaffer (1995) emphasize that quality is meeting the requirements of the customer. According to the International Organization for Standardization (1994), quality is the totality of characteristics of an entity that bear on its ability to meet the stated or implied needs.

It is difficult to define quality in a construction environment because of the large number of variables that contribute to quality. The responsibility for attaining quality lies with more than one party (Rwelamila and Wiseman, 1995). In a study conducted by Harvey and Kagan (1990), panellists consisting of owners, contractors and designers were asked for their opinion on the need for quality control. The panellists agreed that quality control is essential in construction and it is worth the costs to implement. Furthermore, it was said that the approaches used for quality monitoring and control on construction sites were not effective enough to identify defects early in the construction process.

The report written by the CIDB: South Africa (2016) presents an overview of the state of construction quality in South Africa. It was shown that clients are neutral or dissatisfied with the quality of construction on around $20 \%$ of all projects. More or less $12 \%$ of the projects that were surveyed had levels of defects, which were regarded as inappropriate. In the report, it was stated that low- and middle-income residential construction was ranked the lowest in terms of quality achieved.

South Africa makes use of the SANS 1200 (1981) and SANS 2001-CC1 (2007) series of Construction Standards as quality guidelines in construction. However, it was found by FIDIC (n.d.) that a lack of quality in construction is manifested in poor or non-sustainable workmanship, unsafe structures, delays, cost overruns and disputes in construction contracts.

\subsubsection{Quality Assurance}

Quality assurance (QA) was defined by Duncan et al. (1990) as a structured approach to business management and control which enhances the ability to provide products and services consistently to specification, programme and cost. In the study conducted by Harvey and Kagan (1990), QA was defined as the establishment of an overall methodology to assure quality on a project. It starts with the design of the project where the design should have a reasonable likelihood of being successfully implemented.

During construction operations, QA involves looking at mechanisms by which control procedures are established. Moreover, QA was defined as the procedures, criteria and requirements to produce the desired quality and performance verifications (Harvey and Kagan, 1990). Harris and McCaffer (1995) further states that quality assurance concen- 
trates on the production or construction management methods and procedural approaches to ensure that quality is built into the production system.

\subsubsection{Quality Control}

Quality control (QC) was defined by Harvey and Kagan (1990) as the mechanisms of control and the process by which these controls are carried through. Quality control entails checking that all the various stages of the process of serving the customer needs have been conducted correctly and any identified defects have been corrected (Ayandibu, 2010).

According to Zairi (1991), quality control is the operational techniques and activities aimed at both monitoring processes and eliminating causes of unsatisfactory performance of relevant stages of the quality loop in order to result in economic effectiveness. Burke (2007) defines quality control as the method of inspection, in-process inspection and final inspection to confirm that the product has met the required condition. Harris and McCaffer (1995) identifies the major objectives of quality control as follows:

- To ensure the completed work meets the specification

- To reduce clients' complaints

- To improve the reliability of products or work produced

- To reduce production costs.

Authors have different opinions for the meaning of quality control, but a similarity identified was an endeavour to derive a method of detecting and documenting defects and devise means of correction before the product is complete.

\subsubsection{Total Quality Management}

Quality management was defined by the ISO 9000 handbook (1994) as all the activities of the overall management function that determines the quality policy, objectives and responsibilities. The study conducted by Harvey and Kagan (1990) states that the use of QA and QC have great benefits since a serviceable product is produced and mistakes are determined early enough to prevent expensive corrections.

Harris, McCaffer and Edum-Fotwe (2006) state that quality management is primarily concerned with all the activities that take place during a production process or service. Thus, managers have to plan and act as such to implement their strategy. Furthermore, Harris et al. (2006) state that total quality management refers to a process where a product satisfies the needs of a customer or client using both quality control and quality assurance as an aid. It was recommended by Love and Li (2000) that construction companies, as well as consulting firms, implement quality management practices so that the amount of rework in projects can be reduced. 


\subsubsection{Synthesis}

Quality is important in a construction environment. In Section 2.1 it was found that construction projects experienced cost overruns caused by a lack of quality in design and construction processes. Quality assurance is the overall methodology to assure quality on a project, but it is important to implement quality control mechanisms to ensure that the completed work meets the required specifications. Combining quality assurance and quality control results in a quality management system. From previous projects it was determined that the lack of quality management systems is a concern resulting in cost overruns and time delays on projects.

It is important to understand the various aspects of quality. For this study the primary focus is on improving the quality during the construction process. From the information found on quality control it is seen that quality control focuses on the operational stages of a process. Therefore, this study aims to improve the construction process by improving the quality control of structural concrete.

\subsection{Quality of structural concrete}

Section 2.2 focuses on concrete deterioration and the aspects that require good quality control during construction to reduce the degree of concrete deterioration. Figure 2.3 shows a layout of the subsections in Section 2.2 .

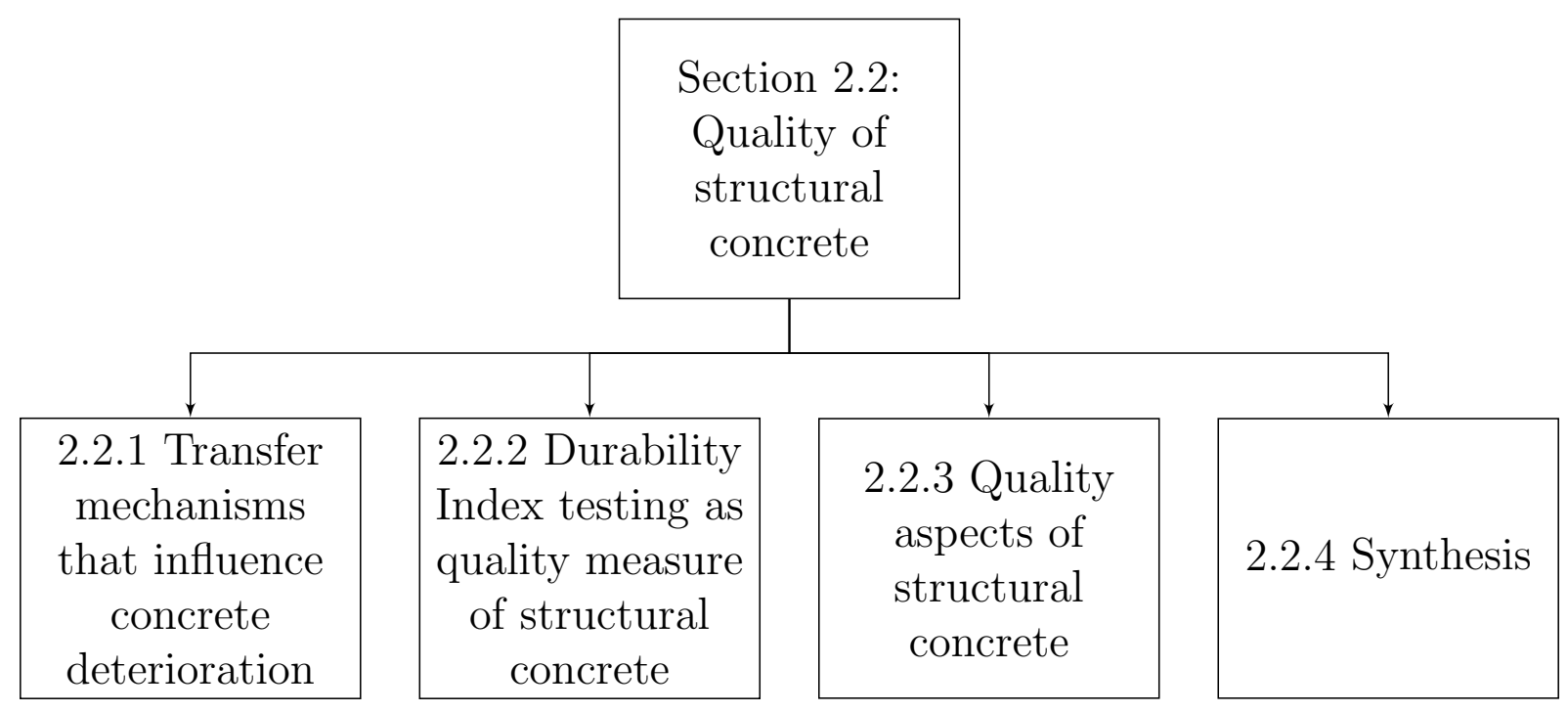

Figure 2.3: Layout of Section 2.2

Section 2.2.1 identifies the various transfer mechanisms that occur in concrete. Some of these mechanisms are diffusion, absorption and permeability. The influence of the transfer mechanisms on the performance of concrete is also identified. The effect of the transfer mechanisms support the argument of the importance of good quality concrete for future durability. 
Section 2.2.2 shows the knowledge gained from the use of the Durability Index (DI) tests. Information from previous studies used the three DI tests to determine the service life of concrete, carbonation depth over time, probability of failure as cover depth changes and the relationship between the cover depth and the corrosion rate.

After stating the importance of quality and its effect on concrete deterioration in Section 2.2.1 and Section 2.2.2, an overview of the conventional construction activities that require good quality control is described in Section 2.2.3.

\subsubsection{Transfer mechanisms that influence concrete deterioration}

The various transfer mechanisms that influence the deterioration of concrete were identified. The transfer mechanisms are influenced by factors that are as a result of poor quality structural concrete. Thus, when construction quality is poor the disadvantageous effect of transfer mechanisms is expected to increase.

The transfer mechanisms that lead to concrete deterioration are diffusion, absorption and permeability. Diffusion is the process by which matter is transported from one part of a system to another due to a concentration gradient. The diffusion of chloride ions into concrete is accompanied by reactions such as physical and chemical binding at the hydration products (Basheer, Kropp and Cleland, 2001).

Water absorption is the transportation of liquids in porous solids due to surface tension acting in capillaries. Absorption is related to the pore structure as well as the moisture condition of the concrete (Basheer et al., 2001). The two basic parameters that determine the absorption of water into concrete are: (1) the mass of water which is required to saturate the concrete and (2) the penetration rate of the capillary rise (Basheer et al., 2001).

Deterioration of concrete is caused by the ingress of various ions, liquids and gases from the environment. The ingress of chlorides or carbon dioxide would depassivate the steel in concrete. In the presence of oxygen and water, steel may start corroding. The ingress of chemicals such as acids, alkalis and sulphates are also responsible for the chemical deterioration of concrete (Basheer et al., 2001).

Carbonation takes place when carbon dioxide diffuses into concrete in the presence of water. The carbon dioxide reacts with the calcium hydroxide which forms calcium carbonate. When carbonation progresses to the surface of the reinforcement, the passive layer of the surface dissolves and corrosion of the reinforcement commences (Basheer et al., 2001).

The fundamental destructive effect of chlorides is their influence on the reinforcement corrosion process. The corrosion risk increases with an increase in the pore solution. Transport mechanisms such as the capillary absorption of chloride-containing liquids, the permeation of a salt solution and the diffusion of free chloride ions can be associated with chloride ingress in concrete (Basheer et al., 2001).

Bleeding is described as the upward movement of water through the concrete towards 
the surface. Bleeding increases the permeability of the concrete and takes place once the concrete has been placed. Permeability of concrete influences the protection of the reinforcement against corrosion (Addis, 2005).

Addis (2005) states that the durability of a material is its ability to remain functional for the desired lifetime of the structure which it is part of. Addis (2005) identifies that when the durability requirements are not met, the effects may be poor serviceability, hazard to persons and property, expensive repair costs and poor aesthetics of concrete.

Transport mechanisms occur in the cover layer of the structural concrete and the cover layer quality is influenced by the construction process Alexander, Ballim and Stanish, 2008). Therefore, it is important to ensure good construction quality to prevent transfer mechanisms from occurring.

\subsubsection{Durability index (DI) testing as quality measure of struc- tural concrete}

Traditionally, concrete strength tests are conducted by measuring the compressive strength of concrete cubes. The test samples are cured and prepared under conditions that bear little resemblance to those in the actual structure. Concrete quality is often dictated by factors other than mixture proportioning. These factors arise as a result of the material quality, handling, placement, consolidation and curing of the concrete (Alexander et al. 2011).

Three Durability Index (DI) tests have been developed that measure the early-age resistance of concrete to the transport of fluids and ions through the cover of the concrete. The durability index approach was developed in South Africa. Alexander et al. (2008) provided steps to establish appropriate DI test values for a given structure and a procedure for implementing their use as a quality control measurement. It was found that improving the quality of a new construction reduces deterioration which then reduces repair costs over the long-term.

The three durability index tests are: (1) oxygen permeability test, (2) water sorptivity test and (3) chloride conductivity test. The parameters that are measured during the tests are the oxygen permeability index (OPI), water sorptivity index and the chloride conductivity index. Alexander et al. (2008) recommend a procedure for implementing a DI test value as a quality control measure. The method introduces a tool that links material properties directly with expected service life. The service life models allow the expected life of a structure to be predicted based on considerations of environmental conditions, cover thickness and concrete quality (Alexander et al., 2008).

The deterioration of concrete is caused by the corrosion of reinforcement bars after the ingress of chloride and carbonation, freezing and thawing, sulphate attack and alkali aggregate reaction. Initial guarantee of durability through service life design approaches and appropriate specification of the as-built quality would motivate clients to pay more for higher initial quality (Alexander et al., 2008). 
Muigai, Moyo and Alexander (2012) conducted a study where the material properties are linked directly with the service life of reinforced concrete. The study conducted by Muigai et al. (2012) focused on South African infrastructure which is mostly situated in coastal areas and exposed to marine environments. The primary cause of deterioration was due to reinforcement corrosion induced by chloride penetrating into the reinforced concrete structure. The deterioration rate depends on the quality and depth of the concrete cover and the chloride concentration required to initiate the corrosion process. Nganga, Alexander and Beushausen (2013) also state that the durability of concrete is not directly related to the strength of concrete, but dependant on the quality of the concrete cover. After chloride penetration, crack formation is caused by active reinforcement corrosion. The final stage involves extensive spalling and loss of steel cross-section. Figure 2.4 shows the corrosion process.

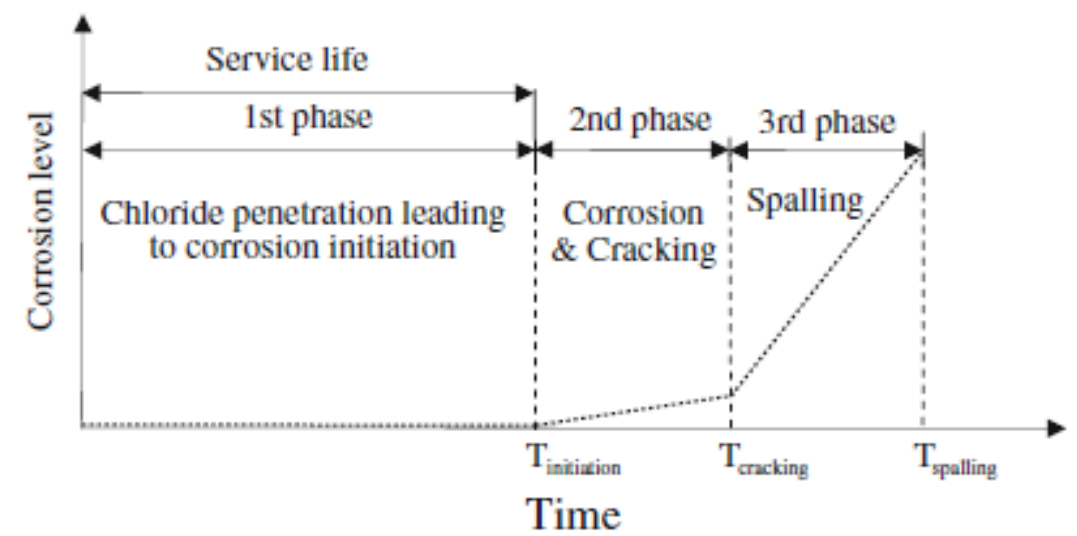

Figure 2.4: Increase in corrosion levels over time (Muigai et al., 2012)

A study conducted by Mackechnie and Alexander (2002) established a strong correlation between the oxygen permeability index (OPI) and carbonation depth, making the test applicable for the prediction of carbonation-induced reinforcement corrosion. The OPI values could be used together with the exposure conditions to determine the carbonation depth over a certain period. When the carbonation depth over a period is more than the cover depth, the cover is not sufficient to protect the reinforcing (Nganga et al., 2013). Figure 2.5 shows an example of using the carbonation depth to determine the service life for various OPI values. 


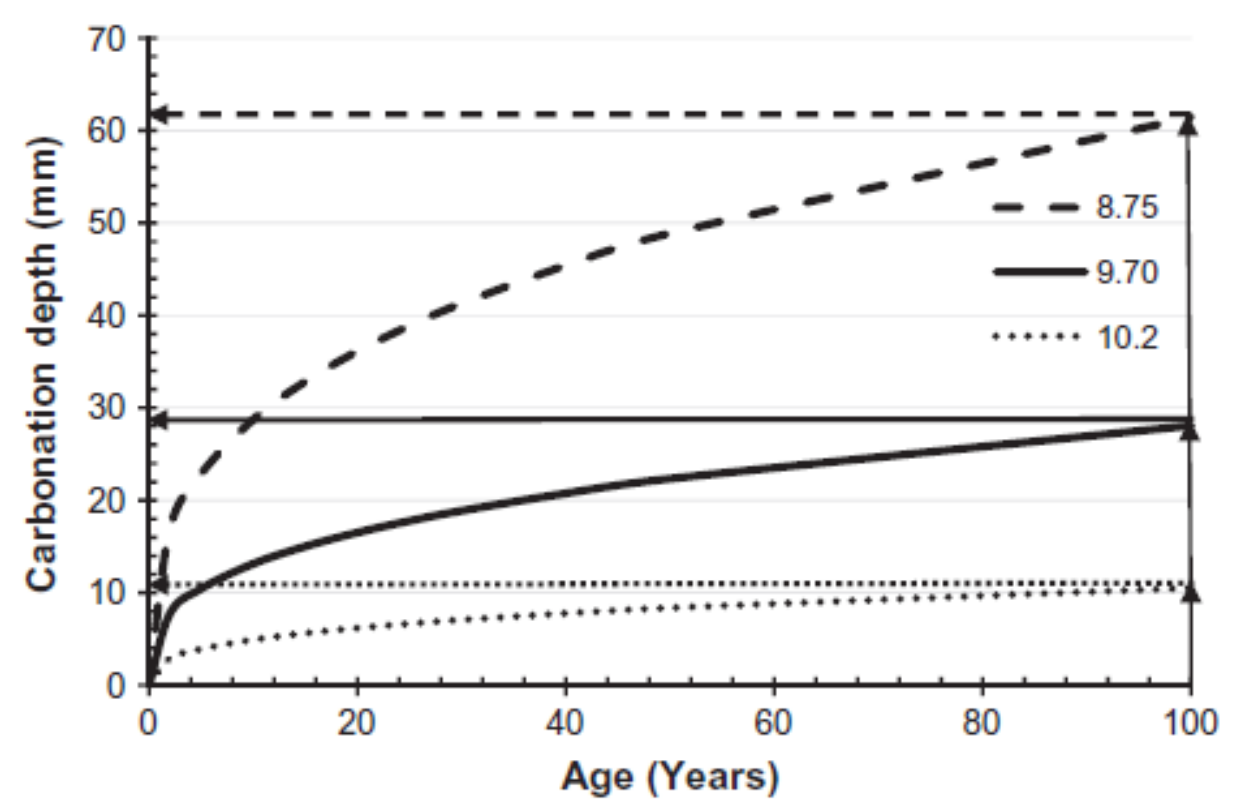

Figure 2.5: Carbonation depth over time for three different OPI values Mackechnie and Alexander, 2002)

Muigai et al. (2012) conducted a sensitivity analysis to determine the sensitivity of probability of failure to changes in cover depth. It was determined that by increasing the cover depth leads to a decrease in probability of failure. The next most influential parameter was established to be the diffusion coefficient, then the critical chloride content and lastly the surface chloride concentration.

Figure 2.6 shows the probability of failure as the cover depth changes. This probability function is only specific to the study conducted by Muigai et al. (2012), but it provides a good indication of how the cover depth influences the durability of the structure.

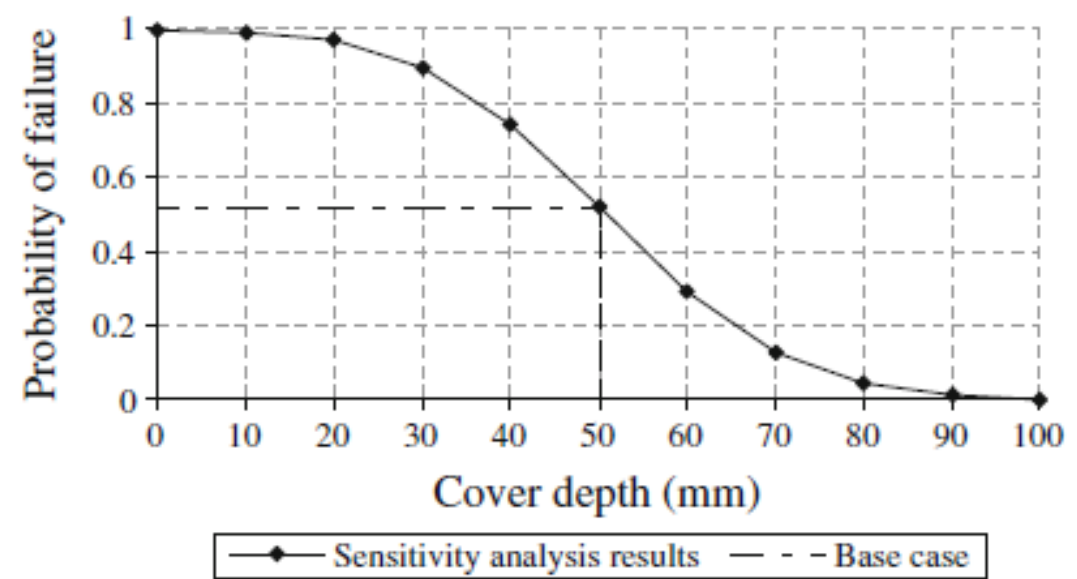

Figure 2.6: Probability of failure for a given cover depth (Muigai et al., 2012)

The corrosion rate is another important input parameter in the corrosion-induced damage models. Scott's model (2004) was developed using results from an experimental set-up 
comprising of cracked beam specimens with $0.2 \mathrm{~mm}$ and $0.7 \mathrm{~mm}$ crack widths having $20 \mathrm{~mm}$ and $40 \mathrm{~mm}$ concrete covers. Scott (2004) used a 90-day chloride conductivity index value. Figure 2.7 illustrates the change in corrosion rate as the cover depth changes for the binder type: CEM I $42.5 \mathrm{~N}$ (PC). It was found that the corrosion rate decreases as the cover depth increases.

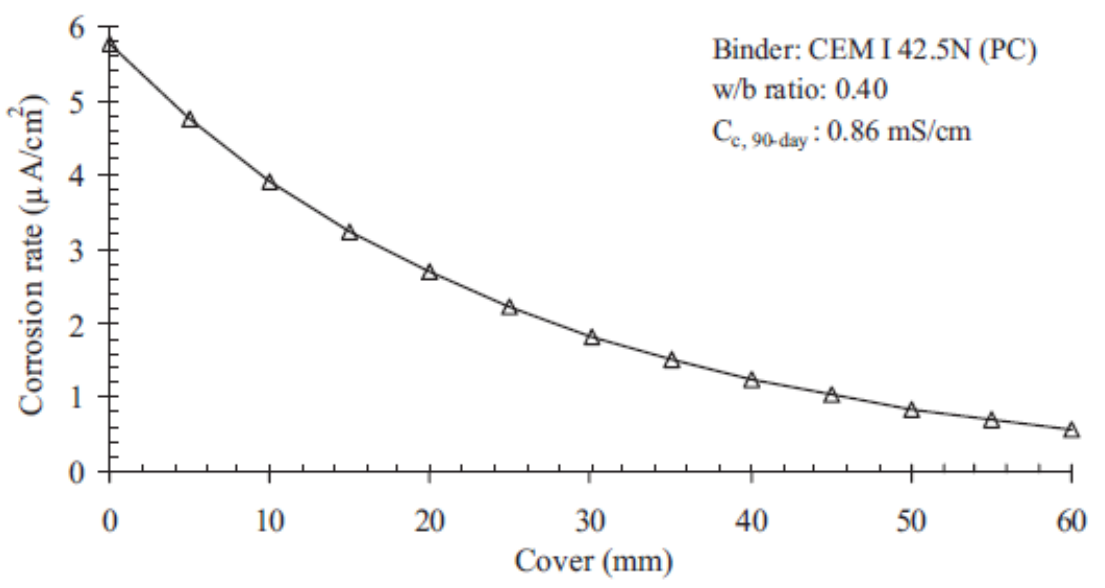

Figure 2.7: Corrosion rate for a given cover (Scott, 2004)

A study conducted by Shammas-Toma, Seymour and Clark (1996) analysed the technical causes that lead to cover not being achieved. Defects were separated into two classes. The first class was defined as management-controllable defects which originate from:

- Impractical design

- Components having been despatched to site being dimensioned incorrectly

- Out of tolerance and inappropriate methods of construction adopted by contractors.

The second class of defects was identified as operative-controllable defects. These defects result directly from operative actions such as misplacement or the omission of components. The study showed that quality management systems were only capable of detecting defects that occur during construction at the point of production. The study showed that the operative-controllable defects constitute approximately $50 \%$ of cover defects.

The information provided in Section 2.2.2 emphasizes how critical good quality construction is to ensure the durability of concrete. It is important that the correct cover quality be obtained during construction. As seen in Figures 2.5 and 2.7, the probability of failure and corrosion rate increases significantly as the cover depth decreases.

Curing also has an important influence on the quality of structural concrete. Appendix A shows the influence of curing on the compressive strength of concrete and the change in carbonation rate as the relative humidity of the concrete increases. 


\subsubsection{Quality aspects of structural concrete to monitor during construction}

From the previous Section it is seen that concrete deterioration is catalysed when the concrete quality is poor. In this Section the various aspects of construction activities that influence concrete quality are identified. It is reasoned that concrete quality is improved through improving the construction quality. Thus, less concrete deterioration would occur if the concrete quality is improved.

\subsubsection{Pre-pour inspections}

Engineers conduct inspection checks on a regular basis to ensure that the construction is completed according to the required specifications. Before the pouring of concrete commences, the following inspections need to be conducted (Martin, n.d.):

- Check that the correct formwork is in place

- Ensure that the correct quantity of shutter release agent is applied

- Ensure that ferule ties are in place and adequately tightened

- Ensure formwork is adequately secured to prevent it from kicking or moving

- Check that the dimensions and alignment are correct and within the specified tolerances

- Ensure that chamfers are in place and adequately secured

- Check that formwork is clean and clear of reinforcement tie wires

- Approve mix and mix design

- Check that existing concrete and construction joints are properly prepared and prewetted.

When considering the inspection checks for the steel reinforcement, it is important to ensure that the reinforcement is placed according to the drawings. The drawing provides the correct spacing, diameter and overlapping distances. The reinforcing should be free of oil. When installing ferule pipes or conduits, minimum clear gaps of $40 \mathrm{~mm}$ are required from the reinforcement bars. Furthermore, a minimum concrete cover of $25 \mathrm{~mm}$ to the pipes is required (Martin, n.d.).

After engaging in a discussion with the resident engineer (RE), during a site visit of a newly constructed sewerage plant in Stellenbosch, the following items were identified as necessary checks during the production of structural concrete (Angelucci, 2016):

- Check that cover is correct

- Check for a clean shutter inside 
- Check that number of bars and spacing correspond to drawing

- Check that lap-lengths correspond to drawing

- Ensure correct level and location of pull-out bars

- Check for clashes with water bar

- Check that shutters are tight at the bottom to prevent grout loss.

In the study conducted by Shammas-Toma et al. (1996), checklists during the production of structural concrete were completed by engineers on-site. The seven common items on all the completed lists that were identified, are as follows:

- Correct distance from starter reinforcement bars to edge of kicker

- Reinforcement ties adequately installed

- Concrete cover spacers are adequate

- Formwork and reinforcement are plumb

- Formwork is stable

- Correct diameter of reinforcement bars and number of bars present

- Adequate cleanliness of reinforcement bars.

Shammas-Toma et al. (1996) identified that many defects remain undetected even when site engineers complete the required inspections. In their study, engineers were interviewed and it was established that they allocate 10-30\% of their time towards checking the work on site. From the interviews it was identified that site engineers sometimes reduce the standard of the quality in favour of time and cost.

From an analysis conducted by Shammas-Toma et al. (1996) it was found that the concrete cover quality does not improve as the number of REs that conduct the inspection increases. The cover quality remained the same when one permanent RE conducted the inspections as well as when a whole team of REs conducted the inspections. This is a possible indication that REs do not complete their work thoroughly or that some inspections are difficult to check by means of the current methods of inspection.

\subsubsection{Placing and compaction of concrete}

Before concrete is placed, the slump is tested. It is tested for each concrete truck that contains a batch of concrete.

Concrete should ideally be placed and compacted in such a manner that segregation is prevented. In the absence of concrete retarders, concrete must be placed within fifty minutes after the mixing process (Martin, n.d.). 
Absorbent surfaces that come in contact with the poured concrete must be wetted before pouring of the concrete commences. However, no standing water must be visible on the surface. Defects are related to the incorrect placement of concrete and the inability of the vibration poker to remove the air effectively from the concrete. Concrete should be fully compacted by means of vibration. Sufficient compaction is important to prevent honeycombing (Martin, n.d.).

\subsubsection{Curing and protection of concrete}

Concrete can be cured by keeping the surfaces moist or by preventing moisture loss by means of a protective barrier that covers the concrete (Martin, n.d.). Curing is important to limit the loss of water from the concrete. Poor curing could result in high creep and shrinkage of the concrete and it reduces the strength of concrete.

Ultimately, poor curing results in the poor durability of concrete since the concrete cover is highly affected by poor curing. Concrete cover is important to provide the reinforcement with protection against deterioration. For more information on concrete curing refer to Appendix A.

\subsubsection{Synthesis}

The presence of transfer mechanisms such as diffusion, absorption and permeability lead to concrete deterioration. Various ions, liquids and gases ingress concrete and cause the corrosion of steel and carbonation of concrete. When steel corrodes, the concrete is more susceptible to cracking. Concrete deterioration influences the durability of concrete. When durability requirements are not met, the effects may be poor serviceability, hazard to persons or properties, expensive repair costs and poor aesthetics.

When the quality of construction is poor, concrete deterioration is accelerated since the concrete is more susceptible to transfer mechanisms. Various quality checks were identified that are currently applied to ensure that the concrete has acceptable quality. However, it does happen that the inspections are not conducted satisfactorily and poor concrete quality remains the result. To improve quality control the barriers that prevent good quality control need to be identified. If the barriers are known then measures can be identified to improve quality control.

In Section 2.3 the barriers toward obtaining good concrete quality are discussed.

\subsection{Barriers preventing good concrete quality}

Section 2.3 provides a range of factors that contribute toward poor quality on construction sites. These factors are better known as barriers that prevent the works from being executed in such a manner that good quality is the result.

Previously, in Section 2.2.3, a range of inspections were provided that are currently implemented to ensure good quality of concrete. Section 2.3 differs from Section 2.2 .3 by 
providing the reasons why poor quality is still the result even when conducting the required inspections.

\subsubsection{Identified barriers in construction}

The current barriers that prevent contractors achieving quality on construction sites were identified by Smallwood (2000) as poor site management, lack of contractor expertise, lack of understanding of quality, inadequate skills and training, lack of quality management systems, reliance on inspections, lack of insight relative to the role of quality and a lack of worker participation.

The CIDB: South Africa (2016) identified barriers that were similar to that of Smallwood (2000). The barriers are poor site management, focus only on time and cost, skills and competence issues, lack of quality improvement processes and the lack of worker participation.

Long, Ogunlana, Quang and Lam (2004) identified various contractor-related problems. They include inadequate experience, construction errors, poor site management and supervision, equipment failures, inadequate labour skills, improper planning and scheduling and poor contract management. Many large contractors are implementing programmes to address their skill requirements, but smaller contractors do not have the resources necessary to address quality factors. The view expressed by stakeholders in South Africa is that the quality delivered by contractors are average, with the quality delivered by Grade 5 to 9 contractors being slightly better than that of Grade 2 to 4 contractors (CIDB, 2011).

It was identified from a case study, conducted on large construction projects in developing countries, that common and general problems of construction projects are grouped under five major factors: (1) incompetent designers and contractors, (2) poor estimation and change management, (3) social and technological issues, (4) site related issues and (5) improper techniques and tools (Long et al., 2004).

In a study conducted by Jergeas and Ruwanpura (2010) in Canada, it was identified that construction is forced to make up for lost time when engineering or material delivery takes longer than scheduled. When a project is fast-tracked, construction problems arise. Jergeas and Ruwanpura (2010) provided a few mismanagement issues within the construction discipline. It was identified that there are inexperienced or poorly equipped project management personnel and supervisors coupled with the inability to understand, plan, adapt, or implement project management procedures. Poor communication, -team work and -alignment exist between the stakeholders and lead to adversarial relationships and protracted disputes. Furthermore, poor site organization and -layout lead to excessive time wastage and loss of productivity during construction.

Duncan et al. (1990) identified cost related errors in construction as follows: (1) poor communication between principal parties lead to confusion and cost related delays, (2) inadequate training and management of labour on site and (3) inadequate verification routines to ensure that design, materials and workmanship meet specific requirements. 
It was also identified that in the process of undertaking work within a tight programme, there seems to be a lack of sufficient time or money to ensure that procedures are correct. Incorporating quality into a system is the responsibility of the client, the design consultants and the contractors (Ayandibu, 2010).

Site practice was identified as an important part that leads to achieving durability. Site practice aspects include the consistency of the mixture, reinforcement in concrete, cover to reinforcement, curing, formwork and compaction (Addis, 2005).

On concrete projects the contractor usually has a shutter installation team, steel fixing team and a team to pour and vibrate the concrete. The teams are susceptible to human influence, applied psychology and occupational health and safety (Mitropoulos and Memarian, 2012). Training was identified as being the most important strategy for an employee to gain knowledge and skills (Tabassi, Ramli and Baker, 2011). A personal interview conducted by Solomons (2014) provided an opinion that $80 \%$ of concrete corrosion problems are due to poor workmanship and $20 \%$ are due to design errors.

In a study by Toor and Ogunlana (2008), respondents of questionnaires rated various factors that lead to success in a construction project. It was identified that effective project planning and control was ranked the highest of the critical success factors. Other important factors identified were competent team members, high quality workmanship, learning from previous experiences, feedback capabilities in the system, clearly written responsibilities, conduction of regular reviews, verification of project progress and the use of up to date technology for automation of construction work. Toor and Ogunlana (2008) identifies that the competence of team members and project managers is a critical factor that leads to project success or failure.

Rework is a negative result caused by poor construction quality. Barriers that contribute toward poor quality were identified by Zhang et al. (2012) as follows:

- Design and engineering: Scope changes, design changes and drawing inaccuracies

- Instruction and inspection: Inadequate supervision and poor communication

- Schedule: Poor rescheduling of resources

- Material and equipment supply: Ill-timed delivery or misplacement and unsatisfactory condition of equipment

- Knowledge: Inadequate knowledge of task at hand

- Skill: Shortages in personnel training

- Self-discipline: No motivation.

\subsubsection{Summary of identified barriers}

Solomons (2014) identified the important barriers that reduce concrete quality. The barriers were identified according to the following categories: 
- Labour

- Management

- Subcontractor

- Working Environment

- Access

- Plant and Equipment

The categories identified by Solomons (2014) are used to sub-divide the barriers identified in this Section. In addition to the barriers identified by Solomons (2014), more barriers are identified in Section 2.3 and are included in a relevant category. The identified barriers are shown according to their relevant category in Figure 2.8. These barriers have an influence on the quality of structural concrete since they are related to the construction errors that lead to rework. 


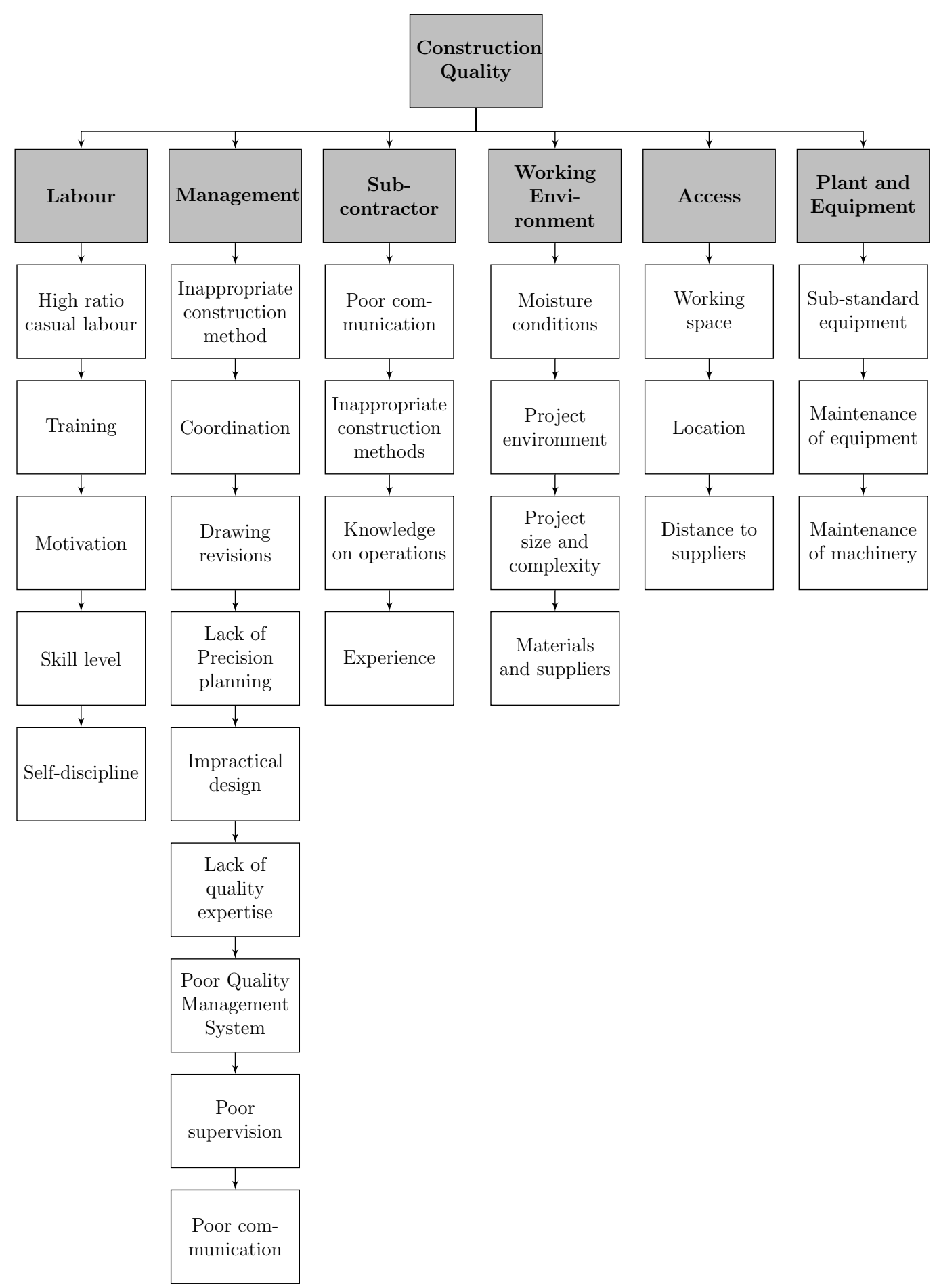

Figure 2.8: Summary of quality barriers that are discussed in Section 2.3

In Section 2.3 the barriers that prevent good construction quality were identified. From various authors' opinions it is found that the barriers are mostly as a result of labour and management attributes. The barriers in Figure 2.8 are as a result of the attributes of labour, management, subcontractors, and plant and equipment. Further in this study the identified attributes are viewed as factors that influence quality expected on a project. If the attributes are expected to be good (less of a barrier) then less construction errors are expected. For example, if the attributes of labour, such as skill level and training, are 
expected to be good then it is expected that less construction errors, such as compaction, are caused by labour.

\subsection{Result of poor construction quality}

This Section looks at the rework costs of poor quality as found by various authors. Furthermore, information is provided on the construction errors that lead to rework of structural concrete.

\subsubsection{Rework as a result of poor quality}

In the construction industry, rework is known as activities that have already been completed, but do not conform to the specified requirements. Therefore, the construction works need to be redone. Furthermore, rework exists due to non-conformance, quality deviations and failure to conform to quality requirements (Zhang, Haas, Goodrum, Caldas and Granger, 2012). Poor construction quality results in rework. Deviations classified as construction errors are the result of erroneous construction methods and procedures (Farrington and Ledbetter, 1992).

In a study conducted by Shammas-Toma et al. (1996), in the United Kingdom, it was identified that corrosion was resulting in the need for millions of pounds worth of remedial work to concrete bridges. It was suggested that future expenditure of this nature could be reduced by improving the quality of workmanship.

There are a number of construction projects that have failed due to poor quality control management. It was identified by the Department of Labour (2002) that the Injaka Bridge collapse occurred due to insufficient reinforcement in the deck section and due to the acceptance and approval of a launching nose which was substantially less stiff than that prescribed in the project specification. There also was a deviation from the project specification regarding the height tolerances of the temporary bearings on one of the piers. Furthermore, the design and construction personnel that were used had inappropriate qualifications and experience. There was a lack of clear instructions in the project specification together with a lack of clear indications on the consulting engineers' design drawings.

Other projects that have failed due to poor quality management was the Pretoria North shopping centre, Coega Bridge and the Investec Office Complex scaffolding collapse (Smallwood, 2012).

A study by Farrington and Ledbetter (1992) analysed the causes of deviations in construction quality. Construction deviations include activities that take place at the project site. The study conducted by Farrington and Ledbetter (1992) showed that in $15.5 \%$ of the total projects studied, deviations were caused by construction errors. The direct impact of the quality deviations on the cost of the total project averaged at $12.4 \%$ of which construction deviations contributed $2.5 \%$. The study only considered the direct costs of quality deviations. 
Harvey and Kagan (1990) proposed a much higher cost of construction rework at $15 \%$ of the project cost. The data gathered was an indication of the impact of the direct costs due to rework. The possibility exists that the secondary impact of construction quality deviations could be much larger than anticipated by Farrington and Ledbetter (1992). It was stated by Smallwood (2000) that rework constitutes $6 \%$ of the project cost for CIDB 5-9 contractors. From these statistics it is reasoned that improved quality control is required to reduce concrete rework.

Lombard (2011) estimated a rework factor by means of a questionnaire survey. Six consultants and four contractors responded to the questionnaire. From the questionnaire results it was found that in South Africa, 15-20\% of total project time is spent on rework and 5-20\% of project cost is spent on rework. Lombard (2011) also stated that on $30 \%$ of South African in-situ projects, construction quality does not comply with requirements. The statistics gathered from Lombard (2011) had a very small sample size and may not provide reliable data.

Akinci, Boukamp, Gordon, Huber, Lyons and Park (2006) found that defects that are detected at a later stage and require rework, result in 6 to $12 \%$ of the total construction cost. Human factors such as unskilled workers and the insufficient supervision of construction work contribute $54 \%$ of construction defects (Akinci et al., 2006).

A field study performed by Smith (2010) noted a large number of non-compliances of beam and slab measurements. $44 \%$ of the slab measurements and $23 \%$ of the beam measurements did not comply with SANS 2011-CC1 (2007). A study conducted by Ronne (2006) investigated the variation in cover to reinforcement of concrete structures in South Africa. It was found that $30 \%$ of the cover data did not meet the specified requirements. Quality of concrete products can be measured by its non-compliance to requirements. Long term non-conformance of concrete results in maintenance or structural failure and short term non-conformance results in rework (Lombard, 2011).

Nganga, Alexander and Beushausen (2013) determined the cover readings from four bridge structures by means of a cover meter. The limit value for cover depth was $40 \mathrm{~mm}$ with provision for minimum cover depth of $30 \mathrm{~mm}$ and maximum depth of $55 \mathrm{~mm}$. The average value for the four structures exceeded the limit value of $40 \mathrm{~mm}$. The minimum values of cover depth were below the acceptable $30 \mathrm{~mm}$ and the maximum values were more than the allowable $55 \mathrm{~mm}$. It was found that $51.4 \%$ of the cover depth readings of one of the bridges were more than the acceptable maximum. When cover depths are less than the minimum acceptable measurement, the cover won't provide adequate protection to the reinforcement against aggressive environmental substances.

\subsubsection{Structural concrete construction errors that lead to re- work}

In Section 2.4.2, the construction errors that frequently occur during the construction of structural concrete works were identified. The construction errors are identified so that improvements in quality control are applied to reduce concrete rework. 
By means of questionnaires and interviews, Solomons (2014) identified the construction errors that have the most significant influence on the quality of structural concrete works in South Africa. The structural concrete works under investigation by Solomons $(\overline{2014})$ included beams, columns and staircases. Solomons (2014) found that the construction errors that occur are caused primarily by labour, management and subcontractors.

From site visits conducted by Solomons (2014), errors to the following construction activities were identified that influence the durability of concrete:

- Bleeding

- Cover to reinforcing

- Steel spacing

- Mix design

- Moisture content of the aggregates

- Compaction

- Grout loss

- Consistency of the mix

- Kicking of the formwork.

During a site visit, Solomons (2014) established that honeycombing occurred in certain parts of the structural elements due to insufficient vibration of the concrete. Grout loss had occurred where columns were not sealed properly. In another site visit, typical problems that affected the concrete finishing occurred where shutters had been reused and not been cleaned properly.

\subsubsection{Summary of identified construction errors}

In addition to the construction errors identified by Solomons (2014), other quality aspects were identified (Section 2.2.3) that could lead to the poor quality of structural concrete if not executed correctly. The construction errors are shown according to the category that is most likely to be responsible for the error occurring. These categories are labour, management, subcontractors, working environment, and plant and equipment. Figure 2.9 shows each of the construction errors. The construction errors represent possible causes of rework during construction. The construction errors in Figure 2.9 are later used in the decision model as risks that could lead to rework. 


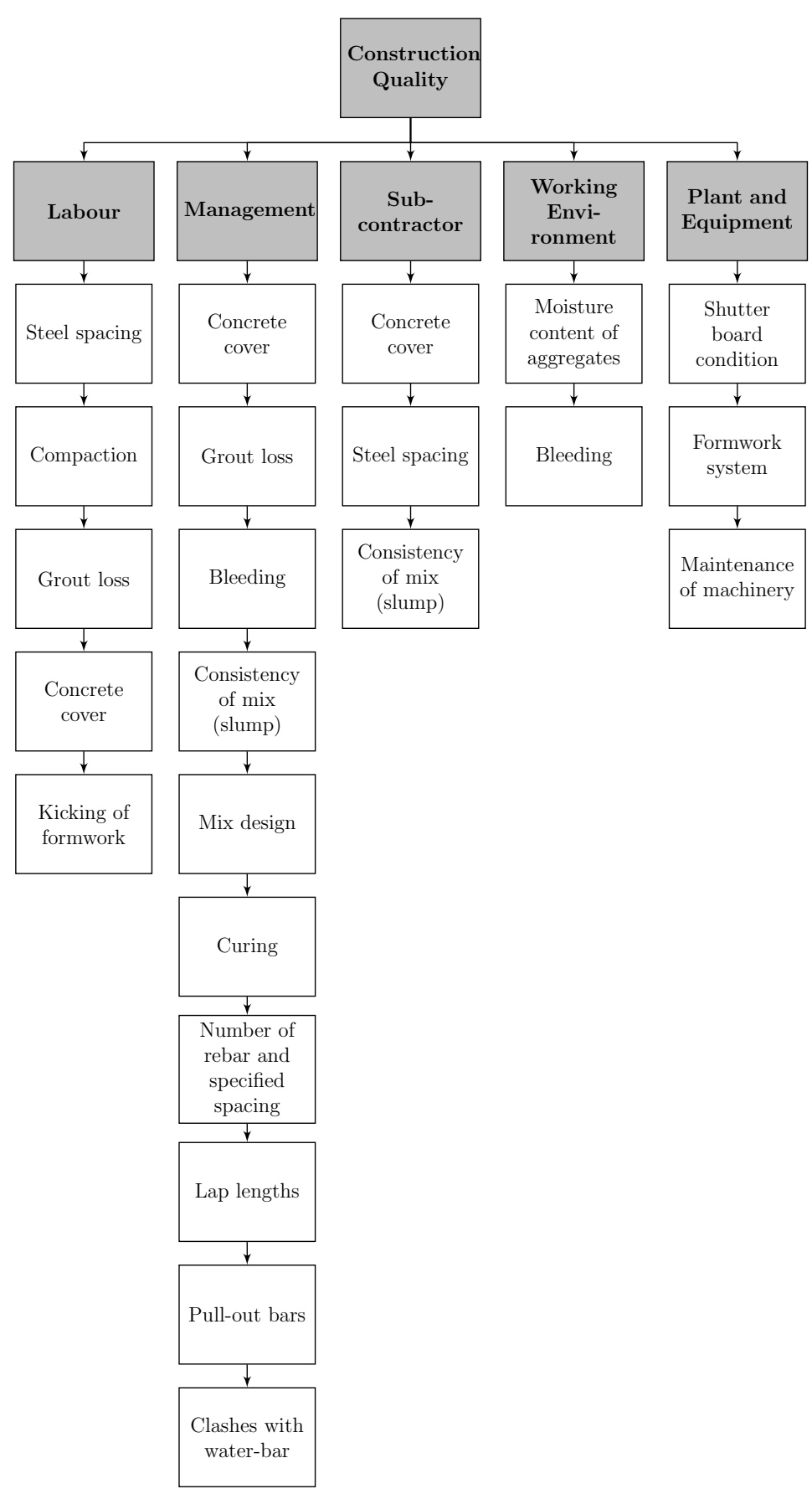

Figure 2.9: Summary of quality checks to ensure good quality concrete

\subsubsection{Synthesis}

A consequence of the barriers that were mentioned by authors is the occurrence of rework on projects. It was shown that rework costs range between 2.5 and $20 \%$ of total project cost. Considering the low operating profit margins on construction projects, excessive rework expenses could easily reduce the financial feasibility of a project.

In Section 2.4.2, Solomons (2014) identified the most influential construction errors that occur whilst constructing structural concrete elements. 
The range of construction errors identified by Solomons (2014) were summarised according to a category that is most likely to cause the error. The construction errors are shown in Figure 2.9.

The influence of the construction errors are used in Chapter 7 to determine the risk magnitude of the construction errors on a project containing structural concrete works.

Construction errors can be reduced by improving quality control during the construction process. The quality control is improved by improving the quality monitoring process. Quality monitoring is discussed in Section 2.5.

\subsection{Quality monitoring}

This Section focuses on the current approaches toward quality monitoring. Quality monitoring is an important procedure to ensure improved quality control. The previous sections showed the errors that occur during construction as well as the factors that influence the expected quality. Later in this study, quality monitoring through use of technology is investigated as a solution to improve the quality of construction.

\subsubsection{Quality monitoring approaches}

Smallwood (2000) investigated systems, shown in Table 2.1, used to achieve quality on projects. The various systems are ranked from best to worse according to the mean rating each system obtained.

Table 2.1: Quality monitoring systems identified by Smallwood (2000)

\begin{tabular}{|c|c|c|c|c|c|c|}
\hline \multirow{2}{*}{ Practice/System } & \multicolumn{5}{|c|}{ Mean score } & \multirow{2}{*}{ Rank } \\
\hline & Client & Designer & $\mathrm{PM}$ & $\begin{array}{l}\text { Grade } \\
5-9\end{array}$ & Mean & \\
\hline $\begin{array}{l}\text { Inspections/Visual } \\
\text { checks }\end{array}$ & 4.21 & 4.67 & 4.17 & 4.43 & 4.37 & 1 \\
\hline $\begin{array}{l}\text { Coordination meet- } \\
\text { ings }\end{array}$ & 3.93 & 4.44 & 4.00 & 4.29 & 4.17 & 2 \\
\hline Client briefing & 3.93 & 4.25 & 3.33 & 4.05 & 3.89 & 3 \\
\hline Samples/references & 3.21 & 4.00 & 3.50 & 4.43 & 3.79 & 4 \\
\hline Checklists & 3.79 & 4.11 & 3.50 & 3.71 & 3.78 & 5 \\
\hline Close out report & 3.79 & 3.71 & 3.17 & 4.05 & 3.68 & 6 \\
\hline Tests & 4.07 & 3.56 & 3.33 & 3.35 & 3.58 & 7 \\
\hline Value management & 3.14 & 3.50 & 2.80 & 4.38 & 3.46 & 8 \\
\hline $\begin{array}{l}\text { Constructability re- } \\
\text { views }\end{array}$ & 3.21 & 3.38 & 3.17 & 3.95 & 3.43 & 9 \\
\hline $\begin{array}{l}\text { Document quality } \\
\text { management system }\end{array}$ & 3.31 & 3.22 & 3.00 & 4.00 & 3.38 & 10 \\
\hline
\end{tabular}


Table 2.1 shows that inspections and visual checks are the best practices to achieve quality on projects. The significance of this result is that the construction participants see value in the use of visual inspection systems. The use of technology provides various possibilities for visual inspection. If the inspection and visual checking system could be improved and implemented through use of technology, the possibility exists for improved quality monitoring on a construction site.

Quality control becomes a complicated process due to the vast size of construction projects. Inspection is performed either through an objective approach or through simple observation. The objective approach is applied to road levels, visual checks, tolerances and fitness for purpose checks. It makes use of measuring devices and equipment to verify that specifications have been met. Simple observations require a well-judged expert opinion (Harris et al., 2006).

Current quality control approaches are not effective, since they only provide data at specific locations and times to present the work in place. The data generated are interpreted manually and are not integrated electronically into the project design and schedule. Akinci et al. (2006) recommends the following for active project quality control: the frequent completion and accurate assessment of the status of as-built conditions at construction sites; identification of critical spatial-temporal and material quality related deviations of work in place and the assessment of whether these deviations constitute of defects.

Automated quality control approaches developed thus far have mostly focused on streamlining the document management process associated with quality assurance and quality management on construction sites. The research conducted by Akinci et al. (2006) identified suggestions for an automated total quality management system composed of quality assurance processes, form libraries and report management modules. Suggestions were also made for a computer-based implementation of ISO 9000 and 9001 guidelines on how to structure and store various quality-related information and documents.

A study conducted by Ballard, Howell and Member (1998) used a production management approach for quality management in construction. In construction, planning is the production of budgets, schedules and other detailed specifications of steps to be followed and the constraints to be obeyed in project execution. After production begins, management devotes its efforts to control, i.e., monitoring of performance against those specifications.

Ballard et al. (1998) identified weekly work plans to meet specific quality requirements for definition, soundness, sequence, sizing and learning. Uncertainty relevant to the management of production on construction processes are interdependent and can radically change project objectives, costs, durations and schedules. The approach used by Ballard et al. (1998) can be useful in any construction project as a baseline for quality monitoring.

A case study conducted by Ayandibu (2010) analysed the quality control approaches that were applied during the construction of the Gautrain in Johannesburg. For this project, there was Independent Certifiers that certified the civil works that were carried out on 
site. Audit checks were used as monitoring tool to ensure that the project plan was being followed (Ayandibu, 2010). According to the Execution Plan that was used for the Gautrain project, equipment for the use of inspection, measuring and testing of works and materials had to be:

- Suitable for the conditions under which the check is being made

- Capable of checking to the required tolerance

- Used by a competent operator

- Of a known reporting accuracy.

It was determined that various authors have different approaches toward quality monitoring. Most of the approaches that were mentioned could make use of technology to improve the process. Research shows that there is a constant seeking towards better quality management systems and methods of improving quality control. Section 2.6 investigates various technological systems that have been used for quality monitoring purposes.

\subsection{Technology available for quality monitoring}

In this Section technology is identified that can be used to improve quality control in construction projects. Technological systems that have not previously been used in construction are also discussed.

The types of technology that were identified are as follows:

- Laser scanners

- Photo- and video monitoring technology

- Point cloud modelling software

- Photo-logging software

- Image dimensioning software

- Internet Protocol cameras

- Remote identification technologies

- Radio Frequency Identification (RFID) tags

- Quick response codes

- Concrete curing sensors.

Each of the identified technological systems are now discussed according to the following format:

- Advantages

- Disadvantages 
- Applications.

In Appendix D, the technical specifications and cost of the technologies that are applicable to this study are shown. The benefits of each of the technological systems for the quality control of concrete in construction, are discussed in Chapter 5 .

\subsubsection{Laser scanners}

3D laser scanning is a non-contact form of measurement and documentation. Investigated models of laser scanners typically collect 250,000 points per second, but can collect up to 976,000 points per second. 3D laser scanners emit laser beams all over the field of view. The laser beam is reflected from surfaces back into the direction of the scanner. The scan data is registered in the form of point cloud data. Point cloud data represents points in a coordinate system consisting of $x, y$ and $z$ coordinates (Surface and Edge, 2011).

Different forms of 3D scanning technologies are suited for short-range scanning, midrange scanning or long-range scanning. Short-range scanners operate at a focal distance (distance between scanner and object) of less than 1 meter and make use of laser triangulation. Mid- and long-range scanners operate at a focal distance that is greater than 2 meters (3D Systems Inc., n.d.).

Using the laser scanner in practice requires the following procedures (Du and Teng, 2007):

- Set up laser scanner to scan the required surface

- Convert the data to a Digital Terrain Model (DTM) format with computer software

- Transfer the data to a notebook or computer with an AutoCAD environment.

\subsubsection{Advantages}

The laser scanner provides a number of benefits. The point cloud registration of the laser scanner is very accurate. It provides high quality measurements in three dimensions with an accuracy of $+/-2 \mathrm{~mm}$ when scanned from a distance of $25 \mathrm{~m}$ (Surface and Edge, 2011). According to Laser Design (2016) an accuracy of $+/-0.0127 \mathrm{~mm}$ could be expected. The scanner provides good colour overlay with less than 1-pixel overlap error.

Sites can be scanned whilst still operational and it increases safety by reducing the surveying time in high traffic areas. Engineers can access scanned data prior to modelling so that preliminary assessments and -calculations could be made. The use of the laser scanner reduces the number of site visits required. The data can be exported in many different formats which enable the integration with many different CAD applications. The scanned images can be uploaded to the internet and protected by a password. This enables the sharing of information with engineers, owners, operators and clients (Surface and Edge, 2011).

Short-range laser scanners are available in many forms such as the area scanner, handheld scanner and the portable arm. The short-range structured light scanners are usually 
more accurate and provide higher resolution. They also provide lower noise levels. Noise is described as the deviation of single readings within sample measurements from the real value. The mid-range and long-range scanners are able to scan at distances between 2-1000m (3D Systems Inc., n.d.).

\subsubsection{Disadvantages}

Short-range laser scanners are more sensitive to surface finishing and may require specific lighting. The mid- and long range scanners have slower data acquisition and cause higher noise levels (3D Systems Inc., n.d.). Training will be required to operate the laser scanner and software is required to transfer the data into a CAD model. The systems are very expensive to buy.

A study conducted by Mani et al. (2009) identified a few disadvantages that go paired with the use of laser scanners. They found that laser scanners are expensive and pose limitations when using them on site. Limitations identified are the discontinuity of spatial information, mixed pixel phenomenon, scanning range and sensor calibration. A reduction in the level of detail of objects have been noticed when they are not close enough to the scanner. The scanners do not provide reliable information on the work sequence and site logistics. In the study conducted by Akinci et al. (2006) these limitations of the laser scanners did not seem to be a governing problem when testing the system, but the amount of time that was spent to create a 3D model was a negative factor.

\subsubsection{Applications}

Various applications of 3D laser scanning exist in civil engineering. Applications include the following:

- Inspection of building façades

- Creation of accurate as-built drawings

- Monitoring of construction progress

- Determination of building maintenance requirements

- Documentation of duct work and piping for clash detection

- The monitoring of bridge deformation over time.

In surveying, the 3D laser scanner allows the user to: create topographical maps; create 3D models and section profiles; measure distances, areas and volumes; map assets and check for clearance; detect construction deficiencies and deformations and produce navigation models for construction planning. The 3D laser scan data allows the user to create a Building Information Model (BIM) (Surface and Edge, 2011). Laser scans are used for re-engineering and design. This makes it possible to add or subtract design features to the existing part or object and use the model for finite element analyses (Direct Dimensions, 2016). 
Laser scanners have been used for condition assessment, component tracking, progress monitoring, and construction quality control. A study conducted by Akinci et al. (2006) investigated the automated quality control approaches developed thus far. Suggestions were made for an automated total quality management system. Akinci et al. (2006) suggests an Advanced Sensor-based Defect Management on Construction Sites (ASDMCon) approach which consists of modules that enable the acquisition and update of the design and schedule information. Furthermore, modules for inspection planning and collection of as-built information together with modules that analyse as-built information from a quality control perspective are enabled. Akinci et al. (2006) used laser scanners for the collection of as-built information while embedded sensors were used to monitor the temperature of the poured concrete. The information was used in a deviation detection system to identify defects and to assess the system through a CAD software system. After identifying and managing defects, the design and schedule information were updated.

The suggested system was applied on three different construction sites and it was found that the technology successfully identified small scale deviations such as changes in wall surfaces, wall thickness and column thickness. The embedded temperature sensors were used to monitor the temperature of concrete over time and it was determined that an unspecified concrete was used on a grade beam. Akinci et al. (2006) concluded that these reality capture technologies have matured sufficiently to be utilized for as-built data collection on construction sites. It is a useful approach to use in project quality control, but future research could further refine the algorithms implemented in support of the formalism and incorporation of other reality capture technologies to improve the automated quality control framework.

Cheng and Teizer (2013) analysed the use of state-of-the-art technology in the field of realtime data collection and visualization. The study conducted by Cheng and Teizer (2013) explains the method of streaming data from real-time positioning sensors to a real-time data visualization platform. The use of digital imagery and visualization materials have previously been used to improve the understanding and assessment of civil- and building engineering. It was found that these methods offered distinct advantages over traditional, instructor-led learning.

Cheng and Teizer (2013) used laser scanning in the survey of the construction site. A commercially available laser scanner was used to collect the as-built conditions of the site. The point clouds of all the scans were registered and used to create a virtual scene. The information was visualized on a remotely located $3 \mathrm{D}$ viewer. Information was published to a server and distributed to multiple users at both local and remote locations in real-time. This aided in fast and correct decision making. Moreover, the virtual reality system can be applied as an education, training and teaching tool. Figure 2.10 shows the sequence of building a 3D virtual model. 

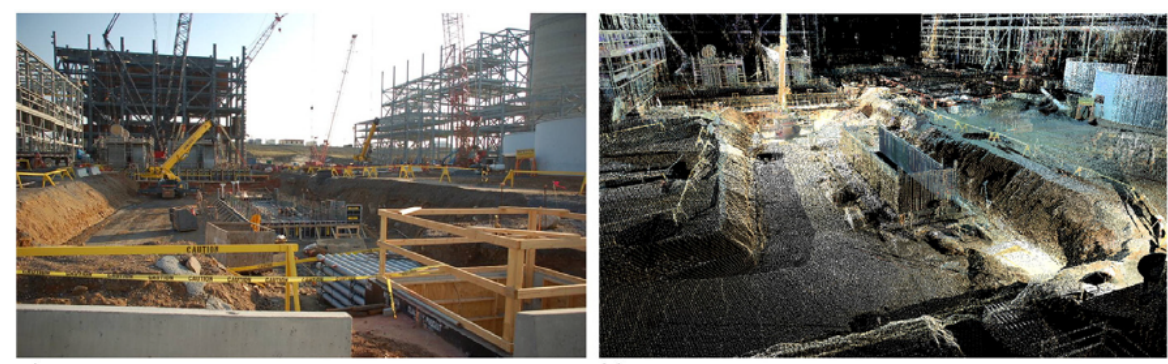

a) Live view of construction site

b) Registered point cloud of laser scans
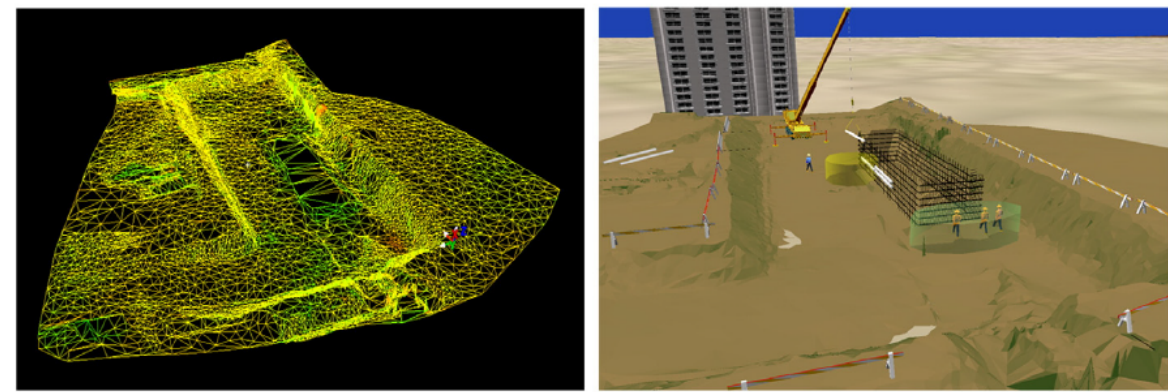

c) Mesh of the terrain

d) Virtual scene including resource models

Figure 2.10: Using a laser scanner to produce a 3D-model of a construction site (Cheng and Teizer, 2013).

This model provided stakeholders with real-time information in an interactive virtual environment and enabled them to observe the construction from a safe position, investigate better perspectives and potentially make faster decisions. Such a model could be used to gather point cloud data on structural elements during construction which could be incorporated into a virtual model during real-time. The model could be used to inspect the quality requirements of structural concrete from a remote location to improve progress monitoring.

In a study by Boukamp and Akinci (2007), 3D as-built data was collected from a nine-story commercial construction project using laser scanners. A colour-coded deviation map was used to highlight deviations associated with as-built components in a given scene. The map was able to identify the horizontal misplacements of columns in the model, but it was necessary to identify the construction specifications applicable to those deviating components and compare the requirements imposed by these specifications to the deviations found.

A study by Wang, Cheng and Sohn (2015) investigated the use of laser scanners to detect the dimensions of precast elements with irregular shapes. By comparing 46 dimensions, it was determined that the accuracy deviation between the as-built and as-designed model showed an average discrepancy of $0.59 \mathrm{~mm}(2.8 \%)$. The maximum discrepancy was $3.4 \mathrm{~mm}$ $(7.2 \%)$. The test was conducted on a down-scaled precast laboratory sample.

\subsubsection{Photo- and video monitoring technology}

Photographs on-site are becoming valuable sources of accurate project information. Digital photos are taken to create a progress photo-log and are utilized for coordination and communication. Cameras and photo-logs have evolved into a significant part of project 
documentation and thus provide solid participations for their usage as visual and realtime technology which does not require any expertise (Mani et al., 2009).

Various technology systems were identified that make use of photograph and video footage. The systems that provide photograph and video monitoring techniques are systems that use cameras to capture data in the form of images or videos. The video footage can be used to monitor site activities in real-time. Images can be logged to support quality control. Computer software can be used to convert images to point cloud models. Mobile application software is available which enables the user to capture images and measure dimensions on the image. The advantages, disadvantages and application of the following systems are subsequently discussed.

- Point cloud modelling from images

- Photo-log software

- Image dimensioning software

- Internet Protocol (IP) camera

- Virtual reality

\subsubsection{Point cloud modelling from images as data input}

Software is used to develop point cloud data from a range of images. The point cloud data is used to develop a high quality textured mesh of the object that was scanned. In summary there are four primary stages executed in the model. The first stage of the process is to capture the images. Stages 2 to 4 are automatically completed by the software. In the second stage the images are calibrated by matching similar points on the various images. In the third stage a dense point cloud model of the object is generated. In the fourth stage the software creates a triangulated mesh of the point cloud data. The mesh enables the model to be textured (Aurvis R\&D, 2014). The result files can be used to measure dimensions that are representative of the real-life object that was scanned.

The process is shown in Figure 2.11: 


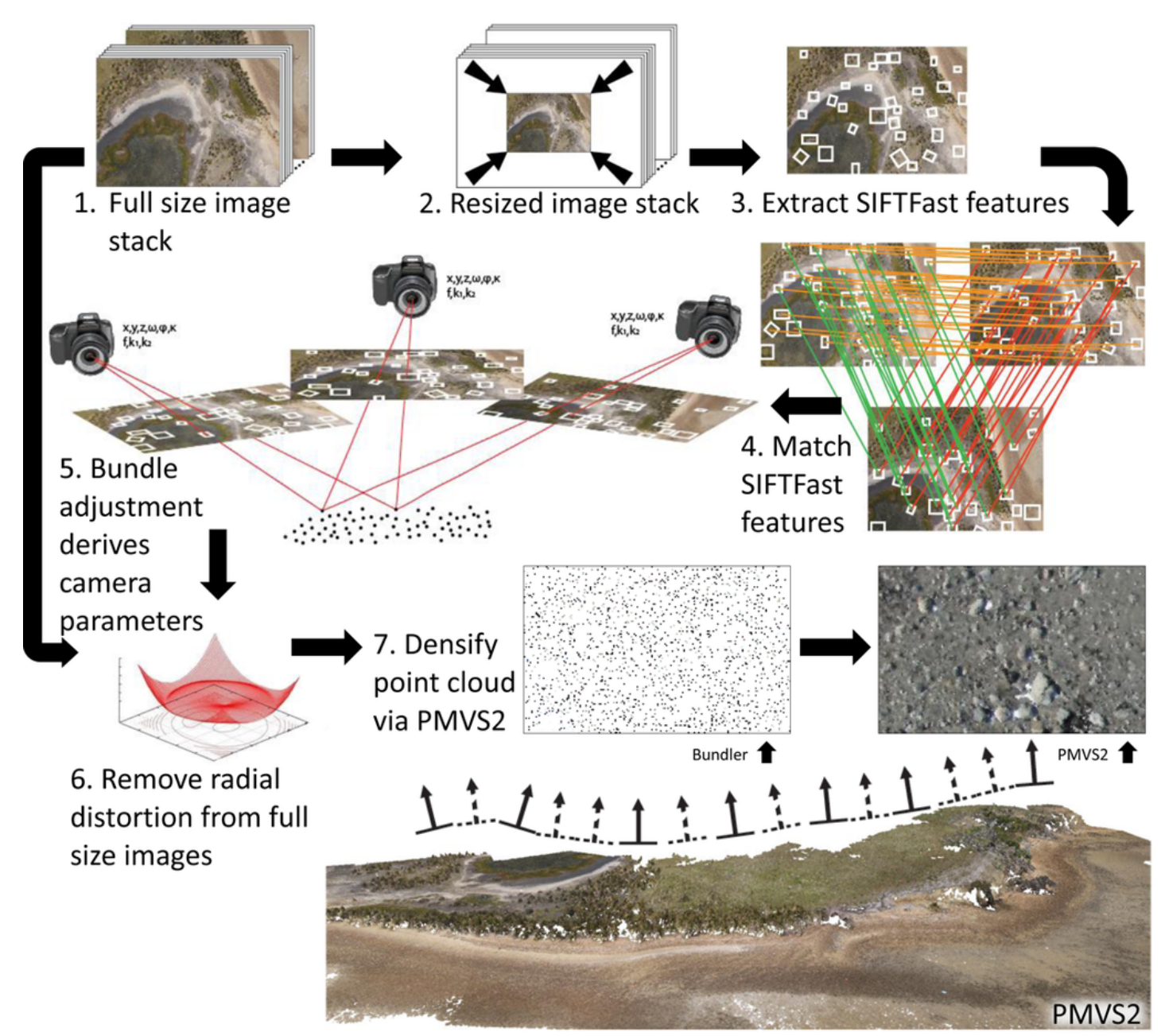

Figure 2.11: Process followed to generate point cloud models from images (Harwin and Lucieer, 2012).

\subsection{Advantages}

The images can be converted to a format that is compatible with CAD. This makes it useful to compare the as-built data with the design of the works. The data captured could easily be shared with a number of stakeholders and would reduce the travel time and number of visits to site. The software enables the user to measure dimensions on the generated model.

A study by Harwin and Lucieer (2012) found that the accuracy of the point cloud data, when capturing images from a UAV at $50 \mathrm{~m}$ above ground level, was within 2.5 to $4 \mathrm{~cm}$. The accuracy obtained is good when considering that the images were captured at a great distance.

Mani et al. (2009) identified various benefits related to the use of augmented reality models. Augmented reality can be identified as when a technology superimposes a computergenerated image on a user's view of the real world. Laser scanners and point cloud models are examples of this. Augmented reality models provide a way for remote construction control decision making; it minimizes the time required to discuss the as-built scene and it 
significantly reduces the travel time and cost required by architects and project executives.

\subsection{Disadvantages}

The accuracy of the model is not as good as that of the laser scanner. The operational manager of Skyler Inc. said that it will be possible to obtain a constant accuracy of more or less $100 \mathrm{~mm}$. This figure exceeds the tolerances specified by SANS 2001-CC1 (2007).

\subsection{Applications}

The availability of good quality imagery provides the opportunity for progress monitoring, study of workspace logistics, quality assurance and safety. The photographs enable 3D visualisation of the as-built scene as well as progress data collection. The combination of $3 \mathrm{D}$ visualisation and progress data is known as a $4 \mathrm{D}$ model.

Visualisation models have been adopted into construction to visualize control data, visualize building products and facilitation of constructibility Three main categories for these types of technologies have been developed to contribute towards the visualization of progress information. These categories are (Mani et al., 2009):

- 4D models as a progress monitoring baseline for simulating as-planned progress

- Colour variations and motions for visualization of progress discrepancies

- Augmented reality for visualization of progress.

4D models are mainly developed for detecting spatial and temporal conflicts, understanding logistics, coordinating construction with subcontractors and visualizing planned progress to owners. The 4D models enable project participants to understand spatial constraints, regardless of their construction knowledge. It is a good underlying platform to monitor progress where deviations between the as-planned and as-built status could be visualized.

\subsubsection{Photo-logging software}

A software application was identified that can be used to log daily inspections on site. The application operates on a mobile phone or a tablet. The application is used to attach images and videos of on-site work onto a cloud-based system. The primary use of the application is for daily reporting and inspections. Stakeholders are enabled to access the site data from their offices (Raken, 2016).

\subsection{Advantages}

The software is used to log weather information, create and assign tasks for team members, add attachments to daily reports (pictures, videos, excel file), send/receive real-time notifications, automatically email construction logs and load daily construction reports onto a cloud storage (Raken, 2016).

The software was tested by the author and showed the following features: 
- User friendly

- Effective tracking of work logs

- View and download images, videos, audio and other files

- Surveys answered on site

- Tasks easily assigned to staff on site.

Such an application serves as an effective manner to handle the daily paperwork required by the Resident Engineer (RE). It also serves as a platform where images can be uploaded and inspections be logged so that quality control is improved.

\subsection{Disadvantages}

The success of the software is dependant on the users. The software constantly requires human input to ensure that there is constant communication between the staff on site and other stakeholders. The process of recording images, videos or audio files could be too time consuming when considering the nature of construction.

\subsection{Applications}

The software is used in construction as a tool for daily reporting. Furthermore, the software is used to log inspections that are completed, provide summary reports of construction to stakeholders, collaborate with subcontractors and dictate notes while inspecting the construction site.

\subsubsection{Image dimensioning software}

Software is available for mobile devices that can be used to measure dimensions, angles and areas on images. The software is low in cost and simple to use. The manner in which the software is used is by capturing a photograph of an object and then measuring the desired dimensions on the object.

\subsection{Advantages}

The computation time of the software is fast and requires low processing power. The software is accurate (see tests in Chapter 4 ) and requires a low initial capital investment to implement.

When disputes arise in the future, due to poor durability of the concrete, the data of the structural concrete inspections can be accessed to assist in the determination of the cause of poor durability. The data is used as evidence that certain construction procedures were executed according to the required specifications. 


\subsection{Disadvantages}

In the plane that the dimensions are desired, an object of known dimensions is required for calibration. For example, when taking a photo of a wall it would be required that an A4 paper (anything with known dimensions) be held in the same plane where the dimensions are required. The A4 paper would be used for calibration between the image and actual dimensions in the plane in which the dimensions are required. The inclusion of a calibration reference makes the process more time consuming and creates difficulty when capturing images in confined spaces.

\subsection{Applications}

Such an application will be useful to capture images on site, which is then uploaded onto cloud storage. The images are downloaded by engineers situated at a remote location. The application is useful to determine dimensions of steel spacing, bar diameter and cover to reinforcement. The images also provide a way of logging inspections that were conducted on site.

Information could not be found where the software was previously used during construction, but tests and recommendations for usage were made by the author in Chapter 4 . The benefits of the software for concrete quality is discussed in Chapter 5 .

\subsubsection{Internet Protocol (IP) cameras}

An Internet Protocol (IP) camera sends and receives data over a Local Area Network (LAN) or the internet. IP cameras require physical cable connections, a Wi-Fi network or a wireless transmission connection to transmit the data. The cameras need to be configured within a network to function. Setting up the network is easy and most of the cameras include their own software to enable recording and viewing on the web (Brick House Security, 2016).

\subsection{Advantages}

The benefits provided by such a system camera is that it enables remote administration from any location; it has digital zoom; it has the ability to send images and video anywhere with an internet connection; it operates at adjustable frame rates and resolution to meet specific needs; it enables two-way communication and it is not expensive (TechTarget, 2016).

\subsection{Disadvantages}

The data has to be compressed when sent over the network to avoid the consumption of too much bandwidth. This reduces the quality of the video by reducing the digital image resolution (Brick House Security, 2016).

On a construction site the works are never at a single location on site. To use the IP cameras effectively it would be required that the cameras capture all the locations of 
construction. If a system is installed that is difficult to move around then the use of the cameras could lead to time wastage.

\subsection{Applications}

The construction of a carbon and stainless steel plant in Alabama made use of wireless networking technologies and scalable IP-based video systems to remotely monitor construction activities on site. The monitoring system was effectively used to minimize the number of on-site visits by project managers. The wireless system was also used to make available recorded videos of the project's progress to stakeholders. The cameras were easily and quickly redeployed as the requirements on site changed (IVC, n.d.).

Various studies provide information where Unmanned Aerial Vehicles (UAVs) were used for inspection through video recording. The UAV video camera works on the same concept as the IP camera system, except that the camera moves around much easier.

A study conducted by Hallermann (2015) investigated a vision-based inspection and monitoring approach for heritage structures based on high quality aerial photos taken by remote controlled UAVs. This approach provides an important contribution to data acquisition processes and existing monitoring strategies. UAVs are equipped with high quality photo or video cameras and are supported by Global Positioning Systems (GPS). The study conducted by Hallermann (2015) focused on the monitoring of heritage monuments, particularly on aerial image based visual inspection and high accuracy survey of structures.

The advanced flight system provides a fast, safe and potentially autonomous data acquisition method without using special access units such as scaffoldings, elevating platforms or professional climbers. In comparison to conventional methods, the UAVs monitoring method showed an increase in the efficiency of inspection and monitoring of heritage monuments by saving time and money for data acquisition (Hallermann, 2015).

\subsubsection{Remote identification technologies}

This section provides an overview of Radio Frequency Identification (RFID) tags and Quick Response (QR) codes. Both types of technology are scanned to obtain information of an object or to register and log an object's location.

\subsubsection{Radio Frequency Identification tags}

Radio Frequency Identification (RFID) makes use of radio waves to communicate. The tags are used to identify and track an object's last scanned location. The system consists of a reader and tags. RFID tags can provide information regarding the description of an object and where it was last scanned. RFID systems are already frequently used in supply chain management, asset tracking and cargo monitoring. The tags are attached to the items that need to be tracked. The tags consist of an integrated circuit (IC) that is connected to an antenna. The memory of the tag stores the information that go paired with the item. The RFID reader is connected to a network and has an antenna that sends power, data and commands to the tags (Impinj, 2016). 


\subsection{Advantages}

RFID systems provide benefits in the supply chain management process with regards to the manufacturing process, warehouse management, tracking and managing of shipping containers, distribution processes and retail benefits (Parvin, 2013). RFID tags do not require correct orientation, such as bar-codes, to be scanned. The tags have a variety of options for data content and read-write capabilities. The tags are much more durable compared to bar-codes (United Parcel Service of America, 2005).

The process identified in construction for which the technology is useful is the distribution of materials. The RFID system allows for products to be scanned and registered when being despatched to construction sites. This allows the contractor to estimate a delivery time of the materials so that construction planning can proceed. RFID has the ability to accelerate the speed of delivery management, improve efficiency and improve the accuracy of delivery management (Parvin, 2013). When products enter the gates of a construction site, the reader technology can register the tags of the items being delivered. The tag has information stored on it, which indicates the material's correct location on site. From this information the materials (reinforcement, cement) are distributed to their correct locations.

\subsection{Disadvantages}

There have been concerns regarding the readability of the tags in their operating environment. Interferences from other devices, temperature, humidity and vibration have a negative influence on the readability of the tags. Metal and liquid objects tend to create problems for reading the tags (United Parcel Service of America, 2005).

A challenging task that was identified with the use of RFID, is to integrate the RFID processes with the existing information systems. (Sabbaghi and Vaidyanathan, 2008).

\subsection{Applications}

Jaselskis, Anderson, Jahren, Rodriguez and Njos (1995) identified various potential applications of RFID technology in the construction industry. Possible applications include concrete processing and handling, cost coding for labour and equipment and materials control.

Parts and containers can be efficiently identified by attaching a tag to each item. This can lead to more efficient material handling and bill-of-materials matching. For construction projects, material identification can reduce confusion regarding the arrival of supplies and their whereabouts at a job site. Refer to the study conducted by Jaselskis et al. (1995) for a detailed explanation on how RFID technology could be incorporated into a system that would ensure proper delivery, billing and quality control for concrete.

The RFID system was found useful in concrete laboratories to obtain information about the status of the cubes during the testing procedure. The information of the cubes and the status of testing is uploaded onto a portal where the engineers and contractors can view the results. A study by Wang (2008) showed that such a system was much more 
efficient to use compared to the traditional method of logging the status of testing. Wang (2008) found that the use of a RFID system increased the efficiency of the monitoring process by $23-28 \%$.

\subsection{Application in Supply Chain Management}

Supply chain refers to all the processes involved in the flow of goods. It is generally considered as a complex and knowledge intensive process and can therefore benefit from the implementation of RFID technology (Parvin, 2013).

The delivery of materials to a construction site was investigated analogous to a supply chain management (SCM) system. RFID systems have been used in supply chain management to improve the effectiveness and efficiency in supply chain problems. The RFID technology ensures the capability to track items in real time. In construction, the RFID system provides a more efficient manner for material handling. RFID will provide the ability for contractors to accurately capture information on the status and location of construction material (Sabbaghi and Vaidyanathan, 2008).

In SCM the use of RFID is useful for order fulfilment and returns management. When ordering products, RFID enables the customer to track the shipping time and shipping location. In construction it would be useful to know exactly when a concrete truck has left the batching plant. The use of concrete that has been produced too long before the arrival on site can lead to the poor quality of concrete.

\subsubsection{Quick response codes}

A Quick Response code is also known as a two-dimensional barcode. The QR code serves as a hyper-link which directs the user to a specific website once the code is scanned. QR codes were originally designed by Toyota to manage parts during manufacturing (Pauley Creative, 2017).

To deploy a QR code system, the following steps are required (Pauley Creative, 2017):

- Select a QR code generator

- Create the code

- Track the QR code.

\subsection{Advantages}

The advantage of the QR code over the bar code is that it can be scanned with a mobile phone. These systems are also advantageous for material-handling on site since they are used in the same manner as RFID tags. The QR code systems are much cheaper than the RFID system. When creating the code on a generating platform, various types of information can be linked to the code. The QR code platforms allow the tracking of the codes to see which codes have been scanned (Pauley Creative, 2017). 


\subsection{Disadvantages}

For each type of QR code, the user needs to program the specific information linked to the code. Scanning the QR codes are more difficult compared to the RFID tags. A clear line of sight is required for a mobile camera to recognise the code, while the RFID tags do not require a line of sight.

\subsection{Applications}

QR codes are already used in the construction industry. QR codes are attached to products which the staff can scan. They are redirected to a website where videos are shown with illustrations of installations (Pauley Creative, 2017).

QR codes are also used in project documentation where the user scans the code to gain additional information on a part of the project (Pauley Creative, 2017).

\subsubsection{Concrete curing sensors}

The curing of concrete is an important process to ensure moist conditions and optimum cement hydration. The hydration of cement increases its strength and reduces the permeability of the cement. Hydration is therefore important to ensure that a durable and strong material is obtained. Refer to Appendix A to view the importance of curing.

Concrete sensors are small electronic devices that are embedded in the concrete. The device provides information that assists in the curing of concrete. Concrete sensors are tied to the steel reinforcement before the concrete is poured. The sensors provide information on the moisture condition in the concrete by measuring the Relative Humidity (RH).

\subsubsection{Advantages}

The advantages of such a system is that it reduces the number of site visits required, it enables the monitoring of the internal temperatures of concrete and it increases quality control during curing. Furthermore, the sensors assist in proposing an optimal time for: (i) the removal of formwork, (ii) the application of load on the structure and (iii) for adjusting the curing temperature of concrete on-site (Structural Health Systems, 2015).

\subsubsection{Disadvantages}

The concrete sensors are buried in the concrete; therefore each sensor can only be used once.

\subsubsection{Applications}

The sensors provide data regarding the temperature and estimated strength of the concrete during curing. The sensors determine whether heating is required during curing on a cold day (Structural Health Systems, 2015).

Traditional methods of obtaining $\mathrm{RH}$ readings require that holes be drilled in the slab and it takes 72 hours for a result to be received. If the sensors were used, the information regarding the moisture condition would be quickly available and synchronized on a 
software application where the project team can access it. The project team is enabled to make timely decisions to avoid additional costs and delays due to rework (Structural Health Systems, 2015). Figure 2.12 shows the wireless concrete sensors.
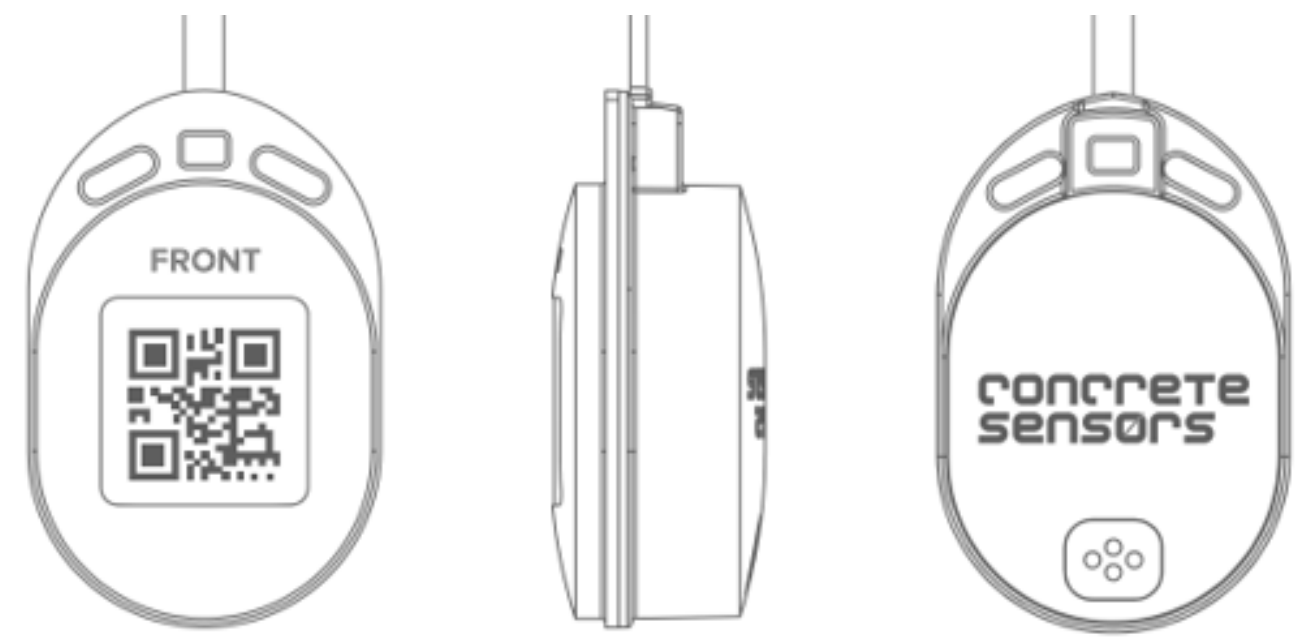

Figure 2.12: Wireless sensors that are embedded in concrete (Structural Health Systems, 2015)

\subsubsection{Synthesis}

Section 2.6 shows technologies that could be used to improve quality control in construction. Various authors have evaluated technologies, such as laser scanners, point cloud modelling software, video cameras and RFID tags. Other technologies, such as photologging software, image dimensioning software and concrete curing sensors, were identified by the author as technologies that have the potential to be used for quality control.

The identified technologies showed various advantages and disadvantages. The laser scanner shows great accuracy for dimensioning applications, however it is expensive to acquire. The point cloud modelling software is low in cost, but the processing time to generate a model is time consuming. The image dimensioning software is low in cost and accurate for dimensioning applications, but the requirement of a calibration reference reduces its user friendliness. The IP cameras have the ability to record high quality footage, but on a construction site it could be troublesome to capture the required footage without occlusions. The remote identification technologies are low in cost and could be very useful to improve the efficiency of material management, but the integration of these systems with existing systems is difficult. The concrete curing sensors provide important information related to the curing of the concrete, but the sensors are not re-usable since they are embedded in the concrete. The technologies show various advantages and disadvantages that need to be considered when acquiring these technologies.

Authors have analysed the use of laser scanners for quality monitoring and comparing as-built data with models pre-developed models. Authors presented cases where the laser technology was used successfully in the identification of small-scale deviations during the construction of walls and columns. 
Authors found that RFID technology was useful for optimising processes in laboratories and tracking materials on-site. Photo- and video cameras were useful in the monitoring of site activities.

However, these technologies have not been evaluated according to its ability to improve concrete quality during construction. Improving quality control would include the monitoring of construction activities to prevent errors, such as incorrect reinforcement spacing, incorrect concrete cover, incorrect concrete vibration and other construction errors shown in Figure 2.9. These technologies are evaluated in Chapter 4.

\subsection{Summary of Literature study}

The Literature study provides the reader with a better understanding of the word "Quality" and how it is relevant in construction. It is shown that quality comprises of quality assurance, quality control and total quality management. Quality control is of importance in this study since it refers to the procedures followed to produce a good quality product during the construction process.

The study aims to improve concrete quality during construction operations. Therefore, the research pertains to processes directly involved in the construction process.

Concrete deterioration and poor durability is a result of transfer mechanisms. When the quality of construction is poor, transfer mechanisms increase. Therefore, improving the quality during construction would improve concrete durability.

Contractors are aware of the quality aspects that need to be monitored to improve concrete quality, however poor quality remains the result. Barriers, that prevent good quality of concrete construction, are present in the construction industry. These barriers are identified as attributes of labour, management, subcontractors, and plant and equipment.

Construction errors were identified that lead to rework during the construction of structural concrete works. For this study, technology is investigated as a possible solution to reduce the identified construction errors. Therefore, technology is considered as a method to improve quality monitoring.

The information found on construction barriers and errors that lead to rework are further used in the development of a decision model. Figure2.1, presented in the beginning of this Chapter, showed the primary areas of investigation in the Literature study. The primary areas were as follows:

- Quality requirements

- Barriers toward obtaining good quality

- Technology. 
Figure 2.13 shows the gap identified in each primary field of research in the literature study together with the gap identified between the three primary fields. The following Chapters aim to fill the gaps.

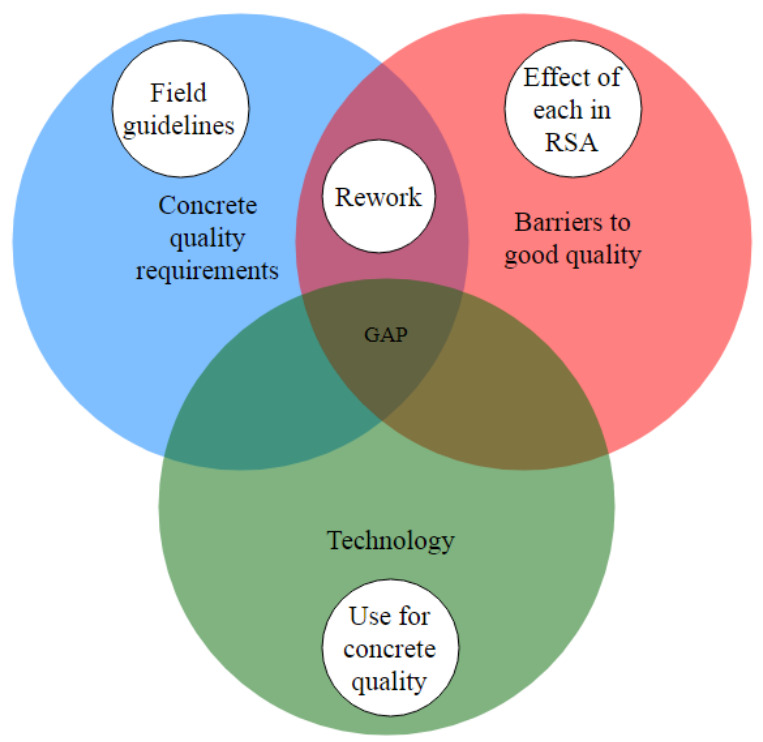

Figure 2.13: Indication of the gap in primary fields of research pertaining to the study

Literature provided sufficient information with regards to the requirements of producing good quality concrete. However, more guidelines and measures are required that could be applied in field to improve concrete quality. This is obtained through interviews.

Furthermore, sufficient information was obtained regarding barriers that prevent good quality construction. However, more information is required regarding the impact that each of the barriers (attributes of labour, management, subcontractors, and plant and equipment in this study) have on the quality of concrete construction. This information is obtained through questionnaire data in Chapter 6 .

When construction barriers lead to construction errors (deviations from quality requirements), rework is the result. More information is required regarding the influence of labour, management, subcontractors, and plant and equipment on construction errors that occur in South Africa. This information is also obtained through questionnaire data in Chapter 6 .

As shown in Literature, technology had been used for various quality monitoring applications in construction. However, a gap was identified which pertains to the monitoring of construction quality during the execution of structural concrete works on site. The technologies identified in literature are further assessed in this study to determine its capability to prevent structural concrete construction errors.

A gap was identified between the crossing of quality, construction barriers and technology. For a technology investment to be feasible it should provide the contractor with economic benefits from using it. For this study a decision model is eventually developed that 
considers the attributes of labour, management, subcontractors, and plant and equipment together with concrete quality requirements to propose a feasible technology investment. The decision model forms the bridge between the specific project conditions and the suitable technology systems for the project. 


\section{Chapter 3}

\section{Research methodology and data collection methods}

The research problem under consideration is the high rework values that occur on structural concrete projects. Through this study the author investigates the use of technology to reduce the rework on concrete. A decision model is developed which considers project attributes in terms of risk. The risk magnitudes are used in the model to assist the user with a technology investment decision. Information that is required in the development of the decision model is as follows:

- Risk magnitude of the primary causes of rework during structural concrete construction

- Capability of currently available technologies to reduce the rework on structural concrete

- Incorporating the information in a decision model that assists a contractor with the investment decision.

This Chapter discusses the research design, data collection and data analysis techniques used in this study. The research methodology is an important process for planning the steps required to achieve the aim of this study. The aim of this study is to develop a decision model that assists a contractor in a technology investment decision to improve the quality of structural concrete.

The steps in the decision model, described in more detail in Chapter 7, together with the data required for each step are shown in Figure 3.1. This should provide the reader with more background of the Chapters to come. 


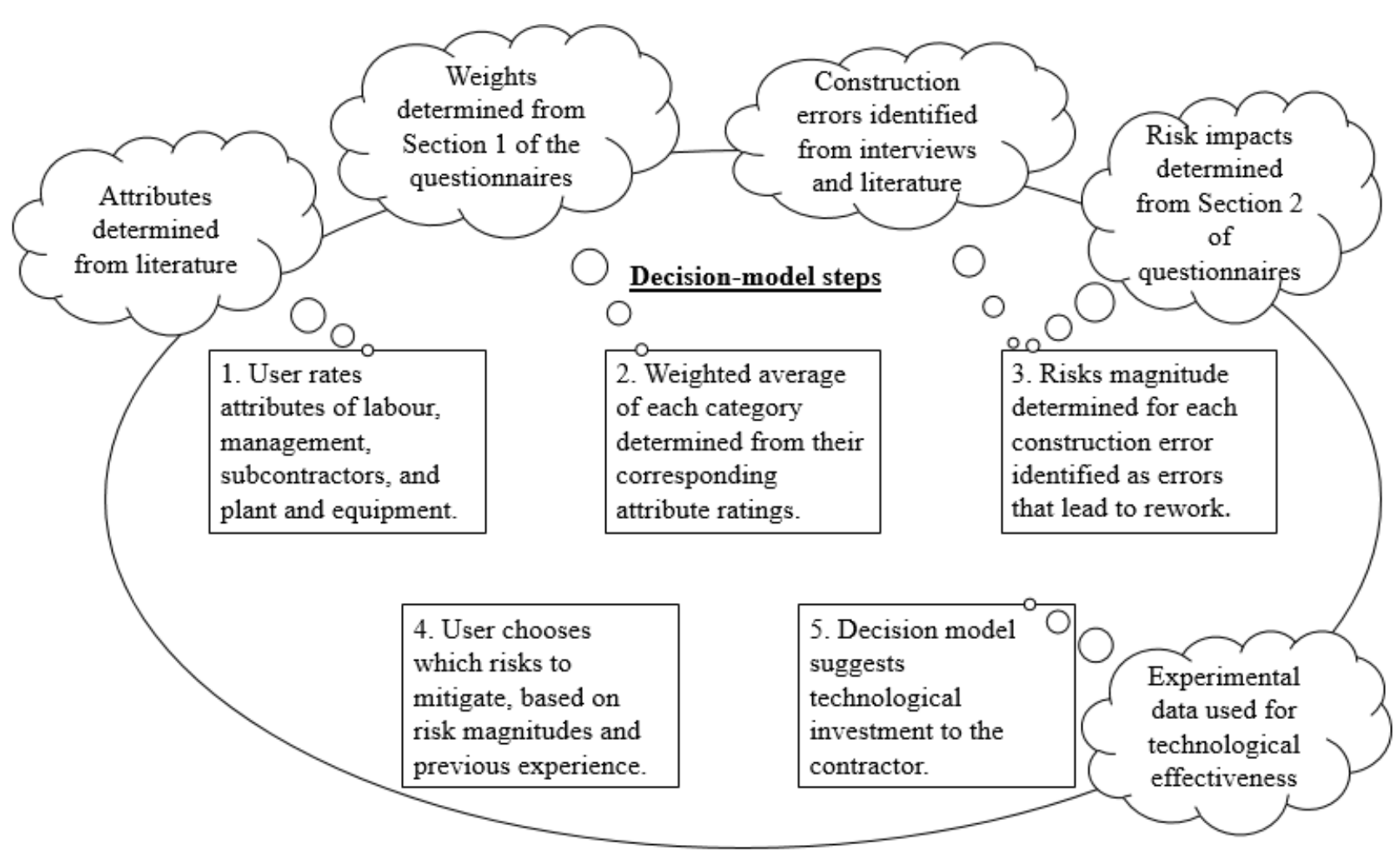

Figure 3.1: Layout of decision model and data required to populate the model

\subsection{Research design}

Research design assists to develop a framework that is used throughout the research study to obtain the data required (Bell, 2005). Leedy and Ormrod (2010) suggest that the following four principal questions regarding research data are answered:

- What data is needed?: Data pertaining to the construction errors that lead to rework and its impact on construction quality. Moreover, preventative measures to reduce construction errors and the technological effectiveness to prevent these errors during construction.

- Where is the data located?: Data will be collected from contractors and consultants in the Western Cape. Technology effectiveness data will be found from laboratory tests and research by other authors.

- How will the data be obtained?: The data will be obtained through a questionnaire survey containing closed-ended questions and through semi-structured interviews. Furthermore, technology data will be obtained through repeated tests in the laboratory. Moreover, human error calculations are done for parts of the information required.

- How will the data be interpreted?: Descriptive and inferential statistics will be used to interpret the data. 
For this study a mixed-method research design is followed. Both qualitative and quantitative data collection approaches were used to achieve the aim of this study. Simulations were conducted to reach conclusions regarding the decision model that is developed.

\subsubsection{Qualitative research}

Qualitative research involves the collection of information that is difficult to reduce to numerical values (Leedy and Ormrod, 2010). In the context of this study, little research was available on measures to prevent construction errors. Therefore, semi-structured interviews with open-ended questions are required to gain more information on the topic.

Leedy and Ormrod (2010) affirmed that the exploratory nature of qualitative research allows the build of theory from the ground up. The semi-structured interviews were conducted to gain more insight with regards to the critical construction errors that lead to rework and how to prevent them from occurring.

\subsubsection{Quantitative research}

Quantitative research refers to research which count things, analyses data statistically and quotes the results in numerical forms (Gomm, 2008). This research method seeks explanations and predictions that will be generalisable (Leedy and Ormrod, 2010).

In the context of this study, quantitative research methods are used to quantify the impact of labour, management, subcontractors, and plant and equipment on the identified construction errors that lead to rework. Furthermore, it is used to determine weights for each of the attributes under labour, management, subcontractors, and plant and equipment that influence construction quality. Closed-ended questionnaires were used to collect the information.

\subsubsection{Simulation}

Simulation is used to represent practical situations mathematically through using key variables that influence concrete quality. The results from the simulations are used to opine on the relevant situations which the model can be used in.

\subsection{Sample size}

A selected number of cases in a population are referred to as the sample size (Walliman, 2005). Where the research study is concerned, data is only required from a portion of the total population (Fellows and Liu, 2008). Leedy and Ormrod (2010) argued that the sample size is dependant on the degree to which the sample includes the characteristics and qualities of the population. When a sample size is $>30$, the data becomes more representative of the population it belongs to (Kaplan Inc. 2015). Therefore, the number of respondents to the questionnaire survey should exceed 30. 


\subsection{Sampling techniques}

In this study the non-probability sampling technique was adopted. In this technique there is no way of guaranteeing that each element of the population is represented in the sample (Leedy and Ormrod, 2010).

However, through purposive sampling, the small sample selected will be representative of the whole population. Participants are chosen for a particular purpose(Kothari, 2004).

In the context of this study, the qualities and characteristics of the participants represent the part of the population that have experience and who's opinion is referred to as an expert opinion. The sample size is limited to the number of responses on the questionnaires. For the interviews the sample size is chosen in such a manner that trends can be established from the answers of participants.

\subsection{Data collection methods}

The data collection methods aim to collect primary- and secondary data.

\subsubsection{Secondary data}

Secondary data refers to data that was found in the literature study. The review of the literature provides valuable information that is used to identify common construction errors that occur during the construction of structural concrete works. This forms the basis of the interviews where the critical errors are identified and mitigation measures are obtained.

\subsubsection{Primary data}

Walliman (2005) states that primary data involves sources that collect data by direct-, detached observation or measurement of phenomena in the real world, where any intermediate interpreter stays uninvolved. In the context of this study, primary data was collected in three stages:

- Interviews

- Experiments

- Questionnaires.

\subsubsection{Interviews}

An interview is a discussion between two people or groups, where the interviewer initiates the interview for the specific purpose of obtaining information pertinent to the researcher's field of study(Best, 1981). An interview is useful to gather information with direct bearing on the research subject and serves as an explanatory device to aid in the identification of variables and relationships. 
Semi-structured interviews were conducted with industry experts to explore the critical construction errors that lead to rework and to identify mitigation measures for these errors. The mitigation measures were used in the evaluation process of the technologies. The interview process contained more than 10 open-ended questions and the duration was more or less 90 minutes for each session. This is shown in Chapter 4 .

\subsubsection{Experiments}

Experiments were conducted in the engineering laboratory at Stellenbosch University. The experiments made use of a laser scanner and a camera. The laser scanner and camera were used to generate point cloud models of a reinforced beam cage. A mobile camera was also used to measure dimensions on photos of the beam cage. The experiments were conducted to evaluate the accuracy, ease of use and processing abilities of the technology.

The researcher reasoned that on site the technology would not constantly yield the same results because of a changing environment. Therefore, the results were determined as if the technology was used under changing conditions. The experimental results were used to derive the technology effectiveness of the laser scanner, point cloud models and image dimensioning software.

The test procedures were as follows:

- Laser scanner

- Set up beam-cage in laboratory

- Measure true dimensions on beam-cage with a 3 meter measure tape

- Set up laser scanner

- Ensure the laser scanner settings are correct (checked by a professional)

- Conduct the scans

- Process the data with software provided

- Measure dimensions in the software and compare with true dimensions.

- Camera to generate point cloud models

- Set up beam-cage in laboratory

- Measure true dimensions on beam-cage with a 3 meter measure tape

- Ensure the camera settings are correct

- Capture 30 images of the beam-cage

- Repeat image capturing process to generate various models and test consistency

- Process the data with software provided

- Measure dimensions in the software and compare with true dimensions.

- Image dimensioning software

- Set up beam-cage in laboratory 
- Measure true dimensions on beam-cage with a 3 meter measure tape

- Place calibration reference in the plane where dimensions are required

- Capture images from various angles to the object with mobile phone

- Measure dimensions in the software and compare with true dimensions.

\subsubsection{Questionnaires}

The nature of a questionnaire is impersonal; therefore, it is important to ensure that the language used is unmistakably clear. It should be designed according to the research objectives. Furthermore, questionnaires should be uncomplicated and not require extensive data gathering (Leedy and Ormrod, 2010).

Questionnaires were used to collect data on the influence of labour, management, subcontractors, and plant and equipment attributes on the quality of concrete. It was also used to determine the degree of the impact that labour, management, subcontractors, and plant and equipment have on the identified construction errors.

The target group for the questionnaire was experts (industry experience $\geq 7$ years) in the contracting and engineering consultancy industry. It was reasoned that these groups would represent the part of the population that has more knowledge on the research field under consideration. Moreover, their experience provides them with knowledge of various types of projects and could provide valuable insight toward the required information.

\subsection{Questionnaire design}

The structure of the questionnaire aims to meet objective number four in Chapter 1. The objective is to "Quantify the risk magnitude of construction errors." The questionnaire is comprised of the following sections:

1. Influence of labour, management, subcontractors, and plant and equipment attributes on the quality of structural concrete.

2. Degree of impact of labour, management, subcontractors, and plant and equipment categories on the identified construction errors that lead to rework on:

(a) Large footings (bases for wind generators)

(b) Structural elements (beams, columns, slabs, reinforced walls)

The data obtained from the first section is used to assign weights to each attribute under labour, management, subcontractors, and plant and equipment. These weights ensure that when the contractor rates these attributes, that the more important attributes contribute more towards the average rating of each category.

The data obtained from the second section is used to quantify the impact that labour, management, subcontractors, and plant and equipment have on the identified construction errors. 
In the decision model a construction risk is identified as a construction error that leads to rework. The likelihood of the error is determined from attribute ratings (user input in decision model) and the impact is determined from questionnaire data (Section 2 of questionnaire).

\subsection{Data analysis}

Data analysis was conducted for the interviews, experiments and the questionnaires.

\subsubsection{Interview data analysis}

The information gathered from the interviews were analysed by means of content analysis. The data recorded from the participants were transcribed and placed in a framework to identify comparisons and contrasts. The data that showed similar trends from the participants were used.

\subsubsection{Experimental data analysis}

The experimental results were quantified by means of a frequency distribution method, namely a histogram. The experimental data was converted to a discrete form; therefore, continuous probability distributions were not used. The data was in a discrete form since a rating was assigned to each experimental value according to a criteria in Chapter 5 . The criteria consists of ratings between 1 and 5. The frequencies from the histogram were used to determine the technological effectiveness in Chapter 5 .

Besides the experimental data, the data used in the histograms include data from other author's studies. The reason therefore was to include as many data points in the histogram as possible. Also, as aforementioned, the data represents various conditions of using the technology. Data from other author's studies were only used to determine the effectiveness of the laser scanner. The histograms were developed using "Statistica", a statistical software package.

Beyond the scope of this study, probability density functions (PDFs) were developed as an additional method to determine technology effectiveness. The data points were used in their continuous form (deviation percentages) and not in their discrete form. The PDFs were not used in the study, but it presents another method to determine technological effectiveness. The PDFs were developed using "Statistica." The PDFs are presented in Appendix C.

\subsubsection{Questionnaire data analysis}

Descriptive statistics (mode, mean, median) were used to interpret the results from the questionnaires. For the first section of the questionnaire, the mean of each data sample was used to derive the weighted rating of each attribute. For the second section of the questionnaire, the mean was used to quantify the impact of the risks under consideration. 
Inferential statistics were used to generalise the findings from the sample to the larger population. Kothari (2004) states that inferential statistics can be used to find out the validity of data with the aim of reaching conclusions. The independent t-test for two samples was used to compare the difference in means between contractors and consultants.

The data samples were validated by determining whether there was a significant difference in the means found from contractors and consultants. It was reasoned that contractors and consultants are involved in different types of work. However, they are involved in a similar working environment. The assumption is made that they are two independent groups. Thus, if the t-test shows similar means for the groups the data is assumed to represent the larger part of the population. The t-test was conducted using "Statistica."

The t-test comprises the following steps(Laerd Statistics, 2013):

- Compute the means $(\mu)$ of the two groups

- Null hypothesis: $H_{0}: \mu_{1}=\mu_{2}$

- Alternative hypothesis: $H_{A}: \mu_{1} \neq \mu_{2}$

- Significance level $(\alpha)=0.05$

- If $\mathrm{p}>\alpha$ then we fail to reject the null hypothesis.

Alpha $(\alpha)$ represents the error that the researcher is prepared to accept. If alpha $=0.05$ the researcher accepts a $5 \%$ probability of rejecting the null hypothesis when it is actually true. This is also known as a Type I error (Kaplan Inc., 2015). The p-value is obtained through the test statistic in "Statistica."

\subsection{Chapter summary}

This Chapter provides more insight regarding the data collection methods that will be implemented to achieve the aim of this study. Qualitative and quantitative methods are required to gather the information required to develop the decision model. Therefore, the data collection will comprise of interviews, experiments and questionnaires.

The information from interviews are determined through content analysis. The data from the experiments is analysed through frequency distributions and the questionnaire data is analysed through descriptive- and inferential statistics.

The layout of the subsequent chapters are shown in Figure 3.2 . 


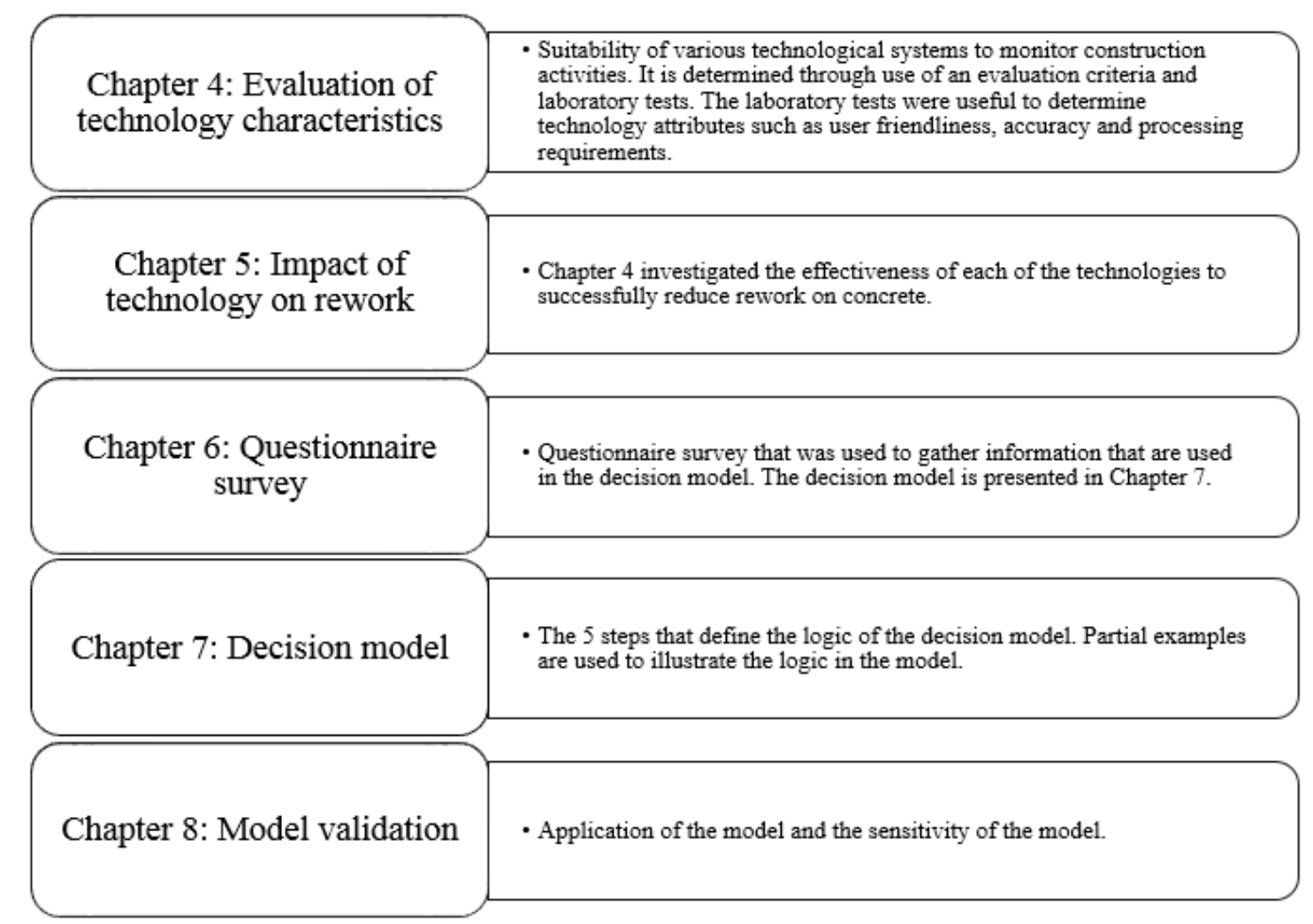

Figure 3.2: Overview of the following Chapters 


\section{Chapter 4}

\section{Evaluation of technology characteristics}

Figure 4.1 shows the scope of content in Chapter 4 compared to the subsequent Chapters.

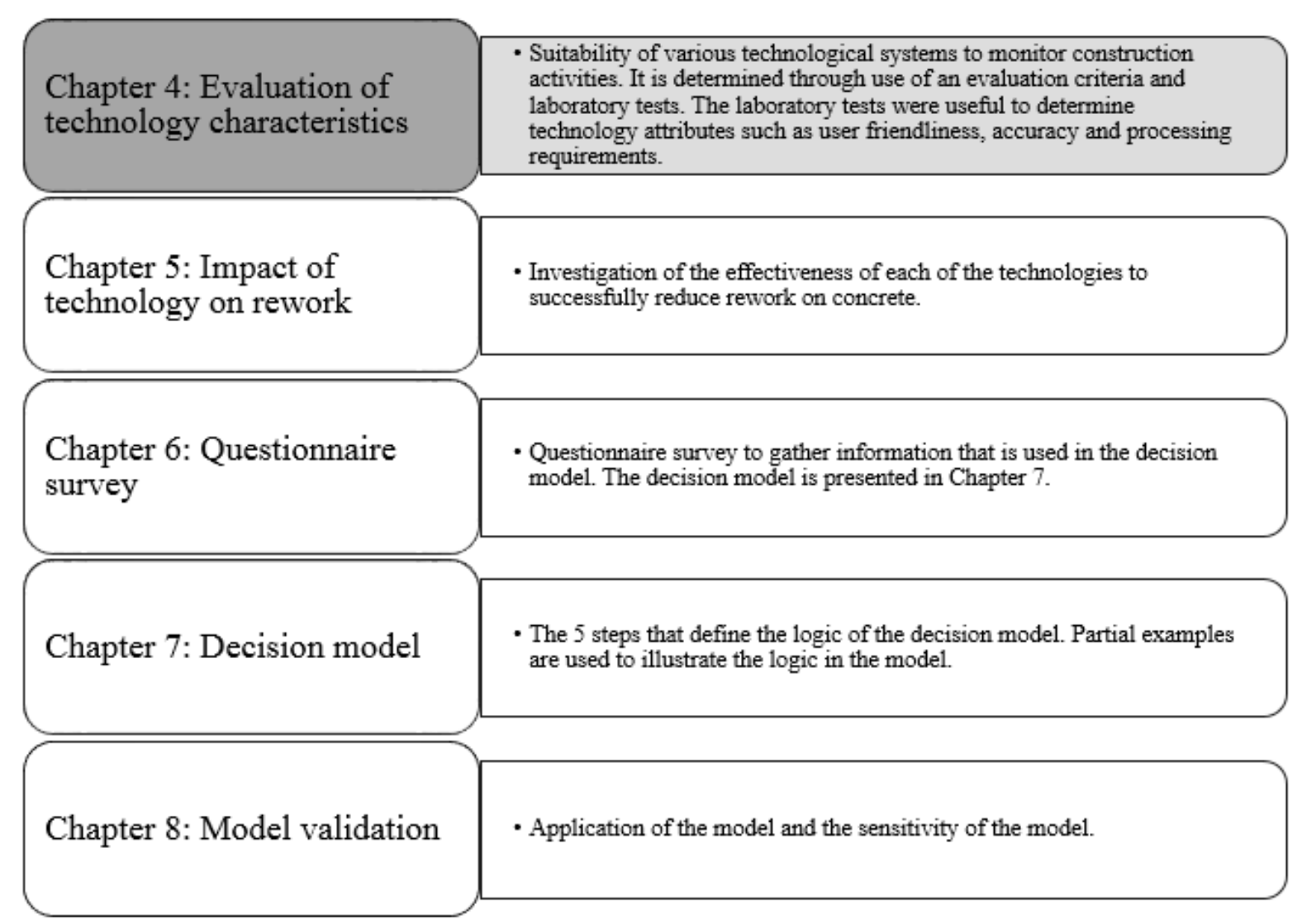

Figure 4.1: Chapter 4 scope

It was reasoned by the author that a sound understanding is required of the construction errors that lead to rework, before evaluating technology to reduce these errors. In this Chapter more information is obtained regarding construction errors and measures to prevent the errors. Interviews were useful to identify the errors that have a more influential impact on the quality of concrete. Furthermore, it was useful to determine measures that 
can be implemented to prevent the occurrence of these errors.

Various technologies are evaluated according to its suitability to assist in the prevention of construction errors. The evaluation is conducted in two steps. In step 1, an evaluation form is completed by the author to identify the construction errors that can be monitored and potentially be reduced through use of the technology. This is shown in Table 4.3. Subsequently, in step 2, tests were conducted with the technologies, in the laboratory at Stellenbosch University (SU), to identify the technology accuracy, ease of use and processing time.

Step 1 and step 2 was conducted for the laser scanner, point cloud modelling software and image dimensioning software. However, only step 1 was completed for the remaining technologies since limitations, such as the availability and proposed purpose of the technologies did not support the laboratory testing objectives.

Figure 4.2 shows an overview of the content in Chapter 4.

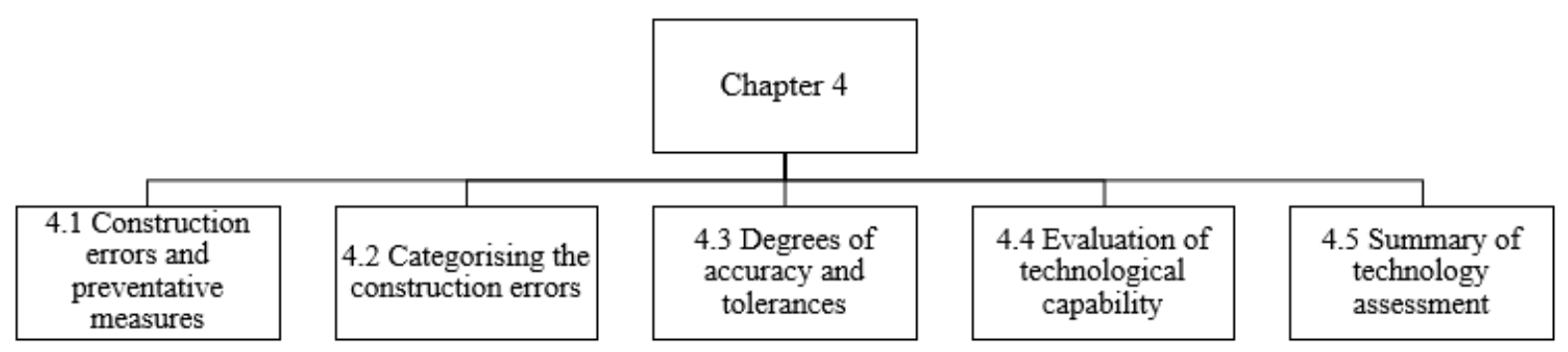

Figure 4.2: Content presented in Chapter 4

\subsection{Construction errors and preventative measures}

Interviews were conducted with three contractors and one consulting engineer. The interviews improved the author's understanding of the construction errors that were identified through the literature study in Chapter 2. Furthermore, the interviews were useful to understand how the errors occur and what preventative measures can be applied. Understanding how to prevent the errors are important if technology is to be considered for that purpose.

Only four participants were interviewed since the information provided by the participants were similar. The most influential construction errors were identified from the interviews and are further used in this study. Therefore, not all of the errors identified in literature are included in the following sections.

Table 4.1 shows the interview participants experience background in the constructionand engineering industry. 
Table 4.1: Experience of interview participants

\begin{tabular}{ccc}
\hline $\begin{array}{c}\text { Participant } \\
\text { number }\end{array}$ & $\begin{array}{c}\text { Years in } \\
\text { construction }\end{array}$ & $\begin{array}{c}\text { Years in } \\
\text { consulting }\end{array}$ \\
\hline 1 & 18 & 3 \\
\hline 2 & 20 & 3 \\
\hline 3 & 7 & 0 \\
\hline 4 & 2 & 25 \\
\hline
\end{tabular}

The construction errors that are further considered in this study are discussed below. The information below was summarized from the interviews with industry experts.

\subsubsection{Steel spacing}

The steel spacing was found not to have such a critical influence on the quality of structural concrete. However, it is important to ensure that enough reinforcement is placed at the point of construction where critical loads are expected. Although it was found that the spacing is not as critical, it remains important for quality control since the steel spacing deviations should be less than the tolerances provided by SANS 2001 - CC1 (2007).

When the spacing is incorrect the concrete develops cracks after one or two years. This could lead to the structure not obtaining adequate durability over its lifetime. When cracks develop soon after construction, a cover meter is used to assess the spacing. If the reinforcement spacing deviation exceeds the allowed tolerances, the contractor would be required to remedy the problem at his own cost.

During construction the spacing is monitored through measuring the actual spacing or counting the number of bars and comparing it to the design drawings.

\subsubsection{Vibration and concrete compaction}

Vibration could lead to poor quality because of under-compaction or over-compaction. In the event of under-compaction, honeycombing occurs. In the event of over-compaction, fines are lost and segregation occurs. It is important to have a compaction team that has adequate experience. Furthermore, incorrect compaction occurs when equipment failures occur during placement. Equipment failures are a result of wear and tear, and poor maintenance. Interviewed participants provided good guidelines for vibration:

- Continue the vibration process if air bubbles continue escaping from the mix as the vibrator is slowly withdrawn from the mix

- Hold the vibrator in the concrete mix for at least 10 seconds at a single location.

\subsubsection{Grout loss}

Grout loss occurs due to shuttering that has not been set up- and sealed correctly. It also depends on the specification of the concrete. A low viscosity concrete is more likely to 
lead to grout loss.

Grout loss leads to honeycombing. Possible preventative measures include the following:

- Foam strips between shuttering

- Sand around the foot of a column

- Foam filler between joints.

\subsubsection{Concrete cover}

Concrete cover is important in structural concrete. Incorrect cover leads to durability problems. There are various factors that lead to incorrect concrete cover:

- Formwork deforms due to temperature

- Incorrect cover blocks

- Not enough blocks cause reinforcing to move during pouring.

Quality inspections are conducted to ensure that there are enough cover blocks and that the correct cover blocks have been used. An inspection is conducted to ensure that the formwork has not deformed, since it would influence the cover to reinforcement.

\subsubsection{Kicking of formwork}

The kicking of formwork occurs when the shuttering has not been braced correctly. The formwork also kicks when the concrete is poured too fast. The pressure from the concrete on the formwork is too high and causes the formwork to move. When formwork kicks, alignment is lost as the formwork twists and moves. Furthermore, if kicking occurs there is excessive grout loss. In most cases it is required that the construction be redone.

To prevent the kicking of formwork the shuttering must be braced correctly and pouring rates should be monitored.

\subsubsection{Bleeding}

Bleeding is caused by excessive power floating. Power floating is the process of smoothing and levelling the surface of the concrete by means of rotating blades. Bleeding occurs when power floating is started too soon. Bleeding also occurs because of excessive vibration. The contractor relies on the experience of the concrete team to ensure that bleeding does not occur. 


\subsubsection{Consistency of the mix (slump)}

Whenever a concrete truck arrives on site, the slump needs to be tested. Problems identified with this process is that the trucks take too long to arrive on site. Unrighteously, water is added to the concrete mix to achieve the correct slump. This reduces the strength of the concrete since the hydration reaction is influenced. One of the participants shared information on a case where a contractor had added water to manipulate the required slump. Cube tests were completed on the concrete and it showed that the mix did not reach the required strength. The contractor had to redo the work, which lead to an additional cost of R2-million. Currently, the process is monitored by means of delivery notes that can be used to determine the exact age of the concrete.

\subsubsection{Curing}

Curing is critical to ensure that the concrete hydration reaction continues for the concrete to develop strength and durability. Curing methods include wrapping or covering the concrete with a plastic; or painting it with a curing compound. A problem identified is that the wrapping is not initially applied or maintained throughout the curing process. The wrapping is often damaged or lost soon after applying it. Problems identified with the curing compound is that the contractor does not keep a good record of which elements have received the compound. This leads to some elements not receiving the curing compound.

\subsubsection{Lap-lengths}

It is important to ensure that reinforcement lap-lengths are correct. One of the participants check all the lap-lengths before pouring concrete. The reason provided was that incorrect lap-lengths could lead to structural failure. The lap-lengths need to be checked and compared with the design drawings.

\subsubsection{Pull-out bars}

Pull-out bars are very important to ensure a strong connection between walls and slabs; as well as between slabs and lift shafts. It is important that the level of the pull-out bars are correct, otherwise an incorrect cover and lever arm can be obtained in the adjacent element. Currently, the correct levels are determined by means of surveying. If the levels are incorrect, the contractor has to dowel new bars into the wall which in turn may not have sufficient anchorage length.

\subsubsection{Shutter boards}

Problems identified with regard to the shutter boards are as follows:

- Shutter boards deform with a change in temperature

- Oil is often not applied to the boards before pouring

- The boards aren't handled with care and are damaged

- Ties aren't cleaned properly before the pouring of concrete 
- Levels aren’t always correct.

When shutter boards deform it causes an uneven finishing and a lack of adequate cover. The oil applied to the shuttering ensures a smoother finishing of the concrete. If the loose ties are not removed from the shuttering then it is embedded in the concrete and must be removed afterwards.

\subsubsection{Formwork system}

The quality of the formwork system will have an influence on the degree to which it deforms under various temperatures as well as how durable the system is to poor handling. Another aspect to monitor during construction is the cleanliness of the formwork.

\subsubsection{Cleanliness of machinery and equipment}

Machinery need to be cleaned regularly to avoid breakages during construction. Breakages lead to a loss of time and additional capital spent on repairing or buying machinery. Maintaining machinery and equipment also increases its life-span. Breakages during construction lead to excessive time loss and poor quality. However, it is dependant on the nature of the breakdown.

\subsection{Categorising the construction errors}

In the previous Section the construction activities are described from which construction errors are likely to result from. Quality monitoring, through use of technology, could potentially reduce rework from the identified construction errors. The construction errors are categorised according to the categories found in the study by Solomons (2014). It is assumed that a construction error would most likely result from the category under which it is placed. The categories are as follow:

- Labour

- Management

- Subcontractors

- Plant and Equipment.

Other construction activities, that were not identified through the interviews, were also included in this study since it is possible to monitor it by means of technology. Furthermore, these activities were identified from site visits as factors that could cause undesirable rework and time wastage if not monitored during construction. The identified construction activities are the following:

- Incorrect dimensions and alignment of formwork

- Incorrect distribution of reinforcement on site (Material-handling). 
It was reasoned that the dimensions and alignment of the formwork could result in error because of the standard and condition of equipment. Therefore, it was considered as an error resulting from the plant and equipment category.

Material handling on site relates to problems that evolve when reinforcement is delivered from suppliers and the reinforcement is distributed to the incorrect locations of the project site. After engaging in a discussion with the Resident Engineer (RE) of a newly constructed waste-water treatment plant in Stellenbosch, it was found that the supervisors have difficulties when searching for the correct reinforcement bundles. This results in the use of incorrect reinforcement on different parts of the construction. Furthermore, time delays are experienced in the process. It was reasoned that this results from poor communication and supervision from management.

Figure 4.3 shows the construction activities that are identified in this study as activities which lead to rework because of construction errors. The construction activities are subdivided between the categories of labour, management, subcontractors, and plant and equipment. 


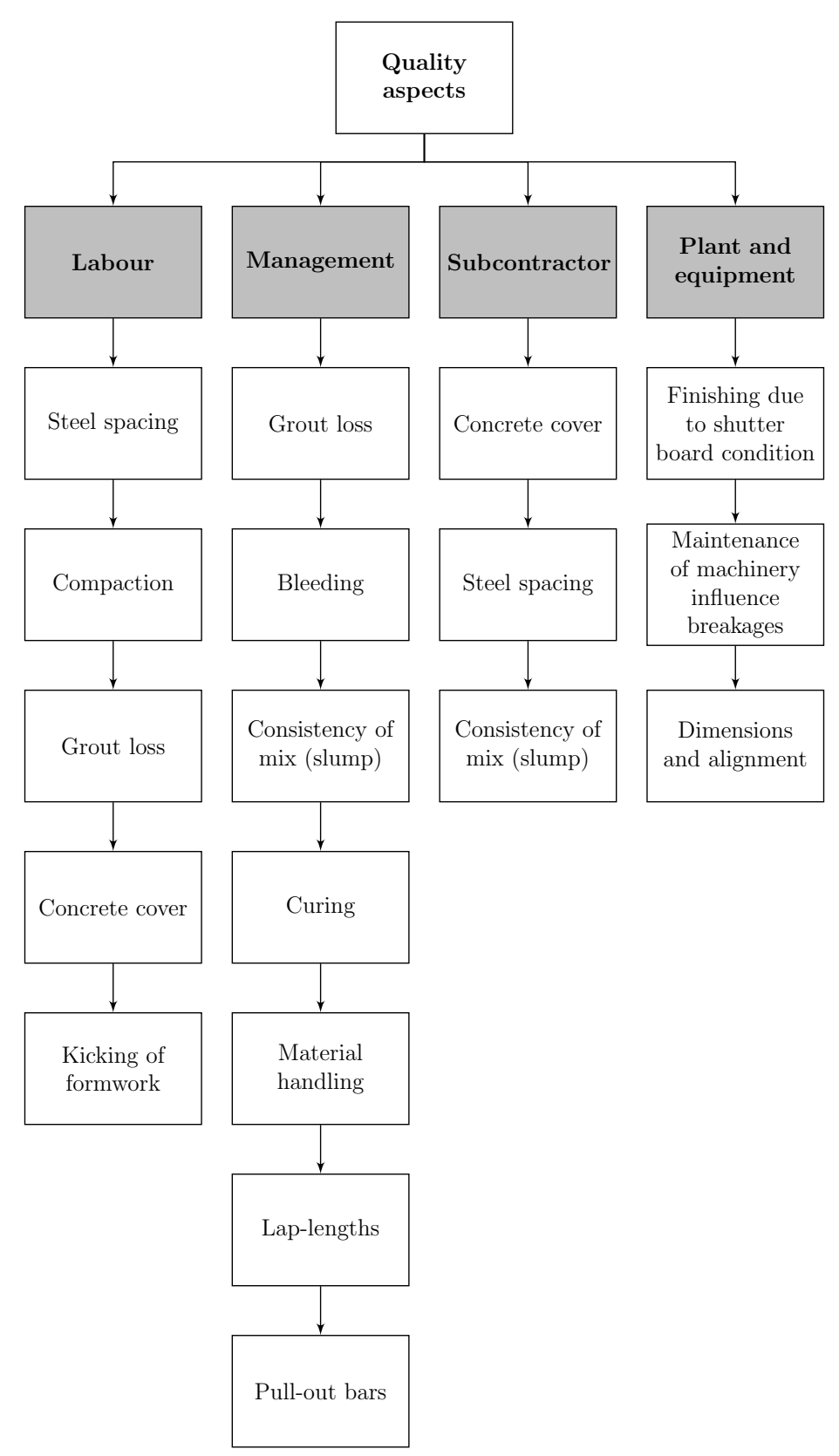

Figure 4.3: Summary of identified construction errors sub-divided under main categories of influence

\subsection{Degrees of Accuracy and tolerances}

SANS 2001-CC1 (2007) specifies tolerances required when building structural concrete elements. SANS 2001-CC1(2007) was used to determine whether the technological systems have accuracies that are within the specified construction tolerances. If the accuracy of a technological system is less than the specified tolerance then the system is not suitable for the monitoring of dimensions.

There are three degrees of accuracy specified in SANS 2001-CC1 (2007). Degree of Accuracy I is suitable for works where the use of special methods or materials is warranted, 
despite the probability of higher costs. Degree of Accuracy II is required for works where a reasonable degree of accuracy is required. Degree of Accuracy III is suitable when a high degree of accuracy is unnecessary. Degree of Accuracy III would normally be for mass foundations (SANS 2001-CC1, 2007). The norm for a Degree of Accuracy III is that exposed surfaces are allowed to have a rough finishing and no treatment of the surface is required after the removal of the formwork. Table 4.2 shows the permissible deviations set out for the three degrees of accuracy.

Table 4.2: Accuracy requirements according to SANS 2001-CC1 (2007).

\begin{tabular}{llll}
\hline \multirow{2}{*}{ Quality aspect } & \multicolumn{3}{c}{ Degrees of Accuracy tolerances (mm) } \\
\cline { 2 - 4 } & I & II & III \\
\hline Steel spacing & $+/-15$ & $+/-20$ & $+/-25$ \\
\hline $\begin{array}{l}\text { Cover to } \\
\text { reinforcement }\end{array}$ & +20 & +20 & +20 \\
\hline
\end{tabular}

Elements above foundation

\begin{tabular}{lccc}
\hline $\begin{array}{l}\text { Cross sectional } \\
\text { dimensions }\end{array}$ & $+5,-5$ & $+15,-5$ & $+20,-10$ \\
\hline Linear dimensions & $+/-10$ & $+/-20$ & $+/-30$ \\
\hline Position on plan & $+/-5$ & $+/-15$ & $+/-25$ \\
\hline $\begin{array}{l}\text { Linear dimensions } \\
\text { on plan }\end{array}$ & $+/-10$ & Foundations & $+/-30$ \\
\hline $\begin{array}{l}\text { Holding down } \\
\text { bolts }\end{array}$ & $+/-3$ & $+/-3$ & $+/-3$ \\
\hline Position on plan & $+/-20$ & $+/-35$ & $+/-50$ \\
\hline
\end{tabular}

\subsection{Evaluation of technology}

The information obtained in the previous Sections of this Chapter is used develop an evaluation criteria for the technological systems. The evaluation criteria assists to identify which technologies are useful for improving the quality control of the construction activities. The capabilities of the various technological systems were evaluated by the author through use of Table 4.3. This is step 1 of the evaluation process. Recall from the introduction of this Chapter that there are two steps in the evaluation process. The second step involves experimental tests in the laboratory.

Both steps of the evaluation process was completed for the laser scanner, point cloud modelling software and the image dimensioning software. These systems are used to di- 
mension various objects during quality control; therefore, the tests were conducted to evaluate the accuracy of the technology. Moreover, factors such as the ease of use and functionality were also evaluated.

The evaluation form, as shown in Table 4.3, was completed for the systems and are presented in Appendix B. The following technological systems were evaluated:

- Laser scanner

- Photo- and video monitoring technologies

- Point cloud models

- Image dimensioning software

- Photo-log software

- Internet Protocol (IP) camera

- Radio Frequency Identification (RFID) tags/Quick Response (QR) code

- Concrete curing sensors.

Table 4.3: Evaluation criteria for technological systems

\begin{tabular}{l|l|l}
\hline Criteria to evaluate capability & Yes/no & Motivation \\
\hline Can the technological system be used to mea- & & \\
sure the spacing between reinforcement? & & \\
\hline Can the system be used to monitor the vi- & & \\
bration process? & & \\
\hline Can the system be used to determine & & \\
whether the vibration is adequate and that & & \\
the correct compaction is obtained? & & \\
\hline Can the system be used to monitor the mea- & & \\
sures taken to prevent grout loss? & \\
\hline Can the system be used to check whether & & \\
cover blocks have been inserted? & \\
\hline Can the system be used to measure the di- & & \\
mensions of the cover blocks? & & \\
Can the system be used to check whether & & \\
actions have been engaged in to prevent the & & \\
kicking of formwork? & & \\
Can the system be used to check whether & & \\
bleeding occurs after power floating the con- \\
crete?
\end{tabular}




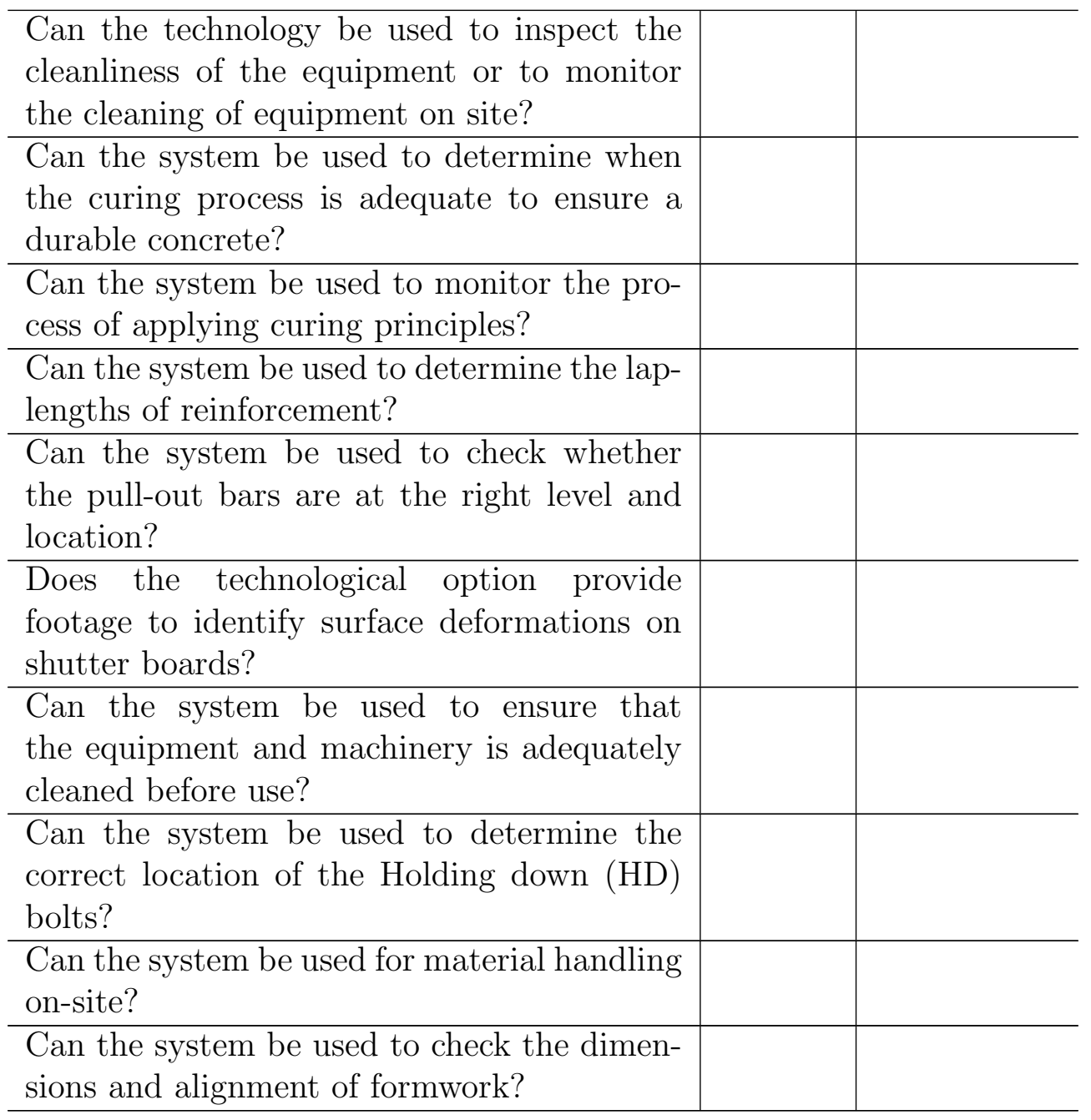

\subsubsection{Laser scanner evaluation}

Laser scanners are used to scan physical objects and then create point cloud models of the objects. Point cloud models are coordinate-based models which enable the dimensioning of the objects that were scanned. The laser scanning systems are only capable of monitoring construction activities where dimensioning or recognition of surface deformation is required. Table 1 in Appendix $\mathrm{B}$ assists in identifying the capability of the system by considering the individual construction activities involved.

The technical specifications of various laser scanners are shown in Appendix D. From the technical specifications it is found that the laser scanner has an accuracy that is within $2 \mathrm{~mm}$. This is within the tolerance requirements stated by SANS 2001-CC1 (2007). Therefore, the laser scanner can be used for the dimensioning of objects during construction.

\subsubsection{Demonstration of the laser scanner}

A company that sells the laser scanner did a demonstration of the product in the Stellenbosch University laboratory. The scanner has a range of $130 \mathrm{~m}$ with high definition view. The object that was scanned is a beam-reinforcement cage. Four scans were completed 
on the medium quality setting (high quality scan duration is too long). The scanning process took more or less 5 minutes per scan and the computer processing took an additional half-hour. Figure 4.4 shows the meshed model generated from the laser scans.

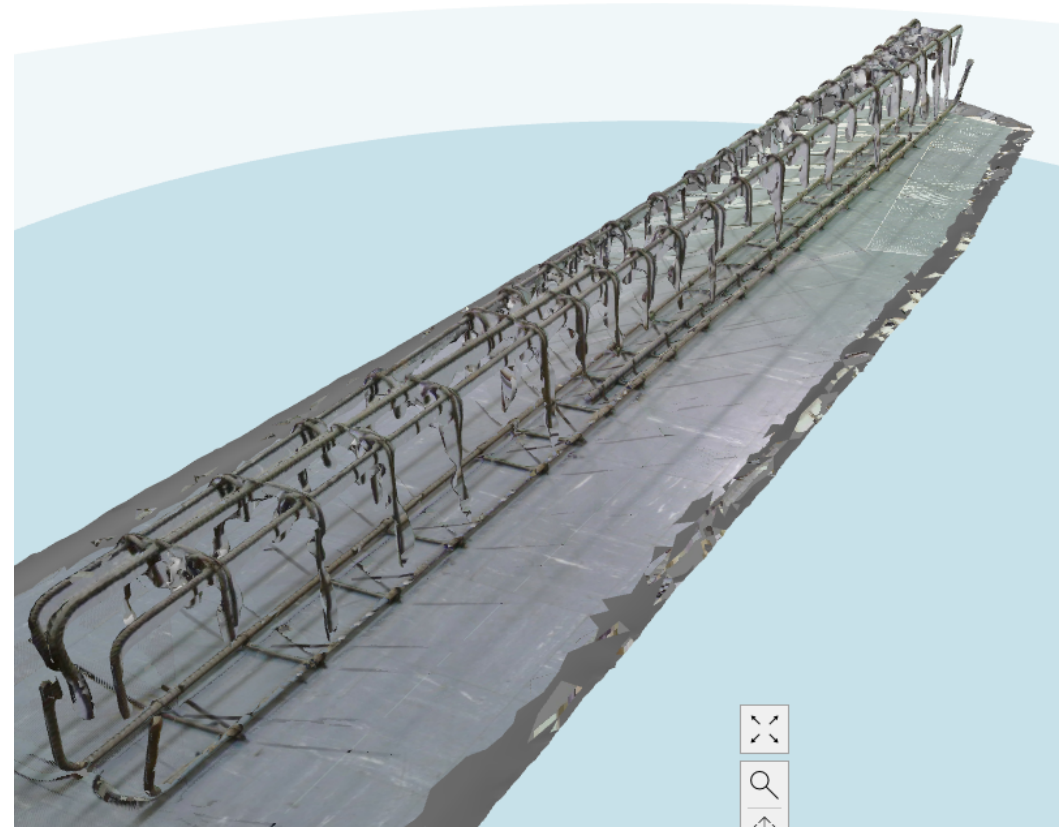

Figure 4.4: Meshed model after using a laser scanner to scan a reinforcement beam-cage

From Figure 4.4 it is seen that there is electronic noise visible on the surface of the object. This is caused by the elevation of the scanner that was too high relative to the beam cage. Therefore, the laser beams could not be reflected from the bottom surface of each bar.

Dimensions can be measured on the meshed model without any prior calibration of the model scale. Table 4.4 shows measurements that were determined through the software and compared to the true dimensions of the beam-cage. Measurement deviations between the software dimensions and true dimensions of reinforcement spacing, stirrup spacing and bar diameter was compared.

Table 4.4: Accuracy deviations determined from laser demonstration data

\begin{tabular}{cccc}
\hline Measurement & $\begin{array}{c}\text { True dimension } \\
(\mathbf{m m})\end{array}$ & $\begin{array}{c}\text { Software } \\
\text { dimension }(\mathbf{m m})\end{array}$ & $\begin{array}{c}\text { Accuracy } \\
\text { deviation (\%) }\end{array}$ \\
\hline 1 & 132.00 & 130.00 & 1.50 \\
\hline 2 & 150.00 & 148.70 & 0.87 \\
\hline 3 & 128.00 & 129.60 & 1.26 \\
\hline 4 & 160.00 & 159.20 & 0.49 \\
\hline 5 & 16.00 & 15.40 & 3.75 \\
\hline
\end{tabular}


From Table 4.4 it is seen that the accuracy deviations for the measurements were small. The largest deviation (3.75\%) was present when measuring the bar diameter (measurement 5). The reason for the large deviation percentage was due to the small dimension of the bar diameter. The scanner is only accurate within $2 \mathrm{~mm}$; therefore, accuracy deviation percentages are large when measuring small objects. Table 4.5 shows the evaluation criteria for the scanner during the laboratory tests. Table 4.6 shows the outcome of the assessment with the scores assigned by the author.

Table 4.5: Evaluation criteria used to assess laser scanner

\begin{tabular}{|c|c|c|c|c|c|}
\hline Criteria & $\begin{array}{l}\text { Very poor } \\
(1 / 5)\end{array}$ & Poor $(2 / 5)$ & Fair $(3 / 5)$ & Good $(4 / 5)$ & $\begin{array}{l}\text { Excellent } \\
(5 / 5)\end{array}$ \\
\hline Ease of use & $\begin{array}{l}\text { Very difficult; } \\
\text { complex }\end{array}$ & $\begin{array}{l}\text { Very difficult; } \\
\text { requires } \\
\text { training }\end{array}$ & $\begin{array}{l}\text { Difficult; gets } \\
\text { better with } \\
\text { training }\end{array}$ & $\begin{array}{l}\text { Easy to use; } \\
\text { interface layout } \\
\text { is logical }\end{array}$ & $\begin{array}{l}\text { Simple user } \\
\text { interface; easy } \\
\text { to operate }\end{array}$ \\
\hline $\begin{array}{l}\text { Accuracy } \\
\text { deviation }\end{array}$ & $<4 \%$ & $<3 \%$ & $<2 \%$ & $<1 \%$ & $<0.5 \%$ \\
\hline Functionality & $\begin{array}{l}\text { Not suited for } \\
\text { intended use }\end{array}$ & $\begin{array}{l}\text { Can be used } \\
\text { for intended } \\
\text { purpose; not } \\
\text { preferred }\end{array}$ & $\begin{array}{l}\text { Usable for } \\
\text { intended } \\
\text { purpose; not } \\
\text { simple enough } \\
\text { to use }\end{array}$ & $\begin{array}{l}\text { Good for } \\
\text { intended } \\
\text { purpose; most } \\
\text { aspects } \\
\text { justified }\end{array}$ & $\begin{array}{l}\text { Software very } \\
\text { suitable for } \\
\text { intended } \\
\text { purpose; all } \\
\text { aspects } \\
\text { justified }\end{array}$ \\
\hline $\begin{array}{l}\text { Data usage } \\
\text { and computer } \\
\text { requirements }\end{array}$ & $\begin{array}{l}\text { Large data files } \\
\text { to process; } \\
\text { requires fast } \\
\text { internet }\end{array}$ & $\begin{array}{l}\text { Medium sized } \\
\text { data files; } \\
\text { requires fast } \\
\text { internet }\end{array}$ & $\begin{array}{l}\text { Data files are } \\
\text { small and easy } \\
\text { to transfer; } \\
\text { requires } \\
\text { internet }\end{array}$ & $\begin{array}{l}\text { Data } \\
\text { processing does } \\
\text { not require } \\
\text { internet; still } \\
\text { need high } \\
\text { computation } \\
\text { power }\end{array}$ & $\begin{array}{l}\text { Software } \\
\text { requires no } \\
\text { internet } \\
\text { connection }\end{array}$ \\
\hline $\begin{array}{l}\text { Time usage for } \\
\text { complete } \\
\text { monitoring }\end{array}$ & $\begin{array}{l}\text { More than } 2 \\
\text { hours }\end{array}$ & $\begin{array}{l}\text { More than } 1.5 \\
\text { hours }\end{array}$ & $\begin{array}{l}\text { Less than } 1 \\
\text { hour }\end{array}$ & $\begin{array}{l}\text { Less than } 45 \\
\text { minutes }\end{array}$ & $\begin{array}{l}\text { Less than } 30 \\
\text { minutes }\end{array}$ \\
\hline
\end{tabular}


Table 4.6: Evaluation of the laser scanner after conducting scans in the laboratory

\begin{tabular}{lcl}
\hline Criteria & Score out of 5 & Comment \\
\hline Ease of use & 5 & $\begin{array}{l}\text { Software very easy to } \\
\text { use. }\end{array}$ \\
\hline Accuracy & 3 & $\begin{array}{l}\text { Accurate to more or less } \\
2 \% .\end{array}$ \\
\hline Functionality & 4 & $\begin{array}{l}\text { Good to use on site. } \\
\text { Very user friendly. }\end{array}$ \\
\hline $\begin{array}{l}\text { Data usage and } \\
\text { computer requirements }\end{array}$ & 4 & $\begin{array}{l}\text { Internet not required for } \\
\text { processing. }\end{array}$ \\
\hline $\begin{array}{l}\text { Time usage for complete } \\
\text { monitoring }\end{array}$ & 3 & $\begin{array}{l}\text { Program processing } \\
\text { speed is slow and } \\
\text { operation is time } \\
\text { consuming. }\end{array}$ \\
\hline
\end{tabular}

From Table 4.6 it can be seen that the accuracy of the device is within $2 \%$ and the software is easy to use. Processing the scans are time consuming and demotivates the use of the device for quality checks that are conducted frequently. No internet is required for the processing. However, it would be required if the model is loaded onto a cloud network so that it can be viewed by stakeholders.

\subsubsection{Capability to monitor construction activities}

The data generated from the scans is useful to measure lap-lengths and the spacing between adjacent reinforcement. The accuracy of the scans are good and enables the user to determine the dimensions of cover blocks. The model generated from the scans enables the user to identify whether concrete cover blocks have been inserted before the pouring of concrete. The good quality scans enable the user to check the level of pull-out bars. Furthermore, the heat maps generated from the scans enable the identification of surface deformation of the shutter boards. In summary, a laser scanner can monitor the following construction activities that lead to construction errors when not executed correctly:

- Steel spacing

- Cover

- Pull-out bars

- Lap-lengths

- Shutter surface condition

- Dimension and alignment

- Position of HD bolts. 


\subsubsection{Photo- and video monitoring}

Various photo- and video monitoring technologies were considered. The different technologies that were assessed according to an evaluation form (step 1) are: point cloud generating software, image dimensioning software and Internet Protocol (IP) cameras.

The photo-log software was not assessed since it is not useful to improve the quality control on its own, but it adds value to the other technological systems. It adds value in the manner that the software platform is useful to assist in monitoring through schedules and inspection lists. Furthermore, it improves communication on site (Raken, 2016).

In this section the author also conducted tests in the laboratory (step 2) to test the accuracy and use of the following:

- Point cloud modelling software

- Image dimensioning software.

\subsubsection{Software that generates a point cloud model from images}

A study by Golparvar-fard, Bohn, Teizer, Savarese and Peña-mora (2011) investigated the accuracy of point cloud models generated from images. Eight experiments were conducted. Four were of column dimensions and the other four of cuboidal masonry block dimensions. Of the four column tests, two were conducted on site and the other two in a laboratory. Of the four masonry block tests, two were conducted outside and the other two inside in a laboratory.

Most of the dimension deviations were more or less $10 \%$. This is very inaccurate when wanting to use the software for dimension measuring. Golparvar-fard et al. (2011) used a camera that does not have very good technical capabilities. The study was conducted in 2011, but technology has improved since then. The camera that was used had a 10.2 effective mega-pixels resolution.

The author conducted own experiments to compare the results with that of Golparvar-fard et al. (2011) when using a camera with improved technical capability. Software was used to create a point cloud model of a beam-reinforcement cage. For this study, tests were conducted by means of a 10- and 16 mega-pixel digital camera as well as a 16-mega-pixel mobile camera. The model generated through use of the 16 mega-pixel digital camera was more detailed and more accurate than that of the 10 mega-pixel camera. The model generated from the mobile phone images was inaccurate, warped and unusable.

The technical specifications of the cameras used in the experiment are shown in Table 4.7. 
Table 4.7: Technical specifications of the cameras used in the experiment.

\begin{tabular}{lccc}
\hline Specification & $\begin{array}{c}\text { Mobile } \\
\text { camera }\end{array}$ & $\begin{array}{c}\text { Digital } \\
\text { camera } 1\end{array}$ & $\begin{array}{c}\text { Digital } \\
\text { camera 2 }\end{array}$ \\
\hline Megapixel & 16.0 & 10.0 & 16.2 \\
\hline Lens Aperture & $\mathrm{f} / 1.9$ & $\mathrm{f} / 3.5$ & $\mathrm{f} / 3.5$ \\
\hline
\end{tabular}

The mobile camera that was used had a 16.0 mega-pixel camera and an aperture of f/1.9. The aperture refers to the opening through which light travels. The aperture determines how accurately aligned the admitted rays are. The aperture of the mobile camera is less than that of the digital cameras that were used; therefore, a lower quality point cloud model can be expected. Digital camera 1 and 2 have similar specifications, but camera 2 has increased mega-pixels

The models were generated from the photographs by capturing 30 photographs of the beam reinforcing with each of the cameras. The photographs were taken from all directions. The model generated from photographs of digital camera 1 was slightly warped and had more deviations compared to the model generated by using digital camera 2 . From the results it was found that the model generated, through use of digital camera 2, showed good accuracy. Figure 4.5 shows a photograph of the cage and the point cloud model next to it when using digital camera 2.

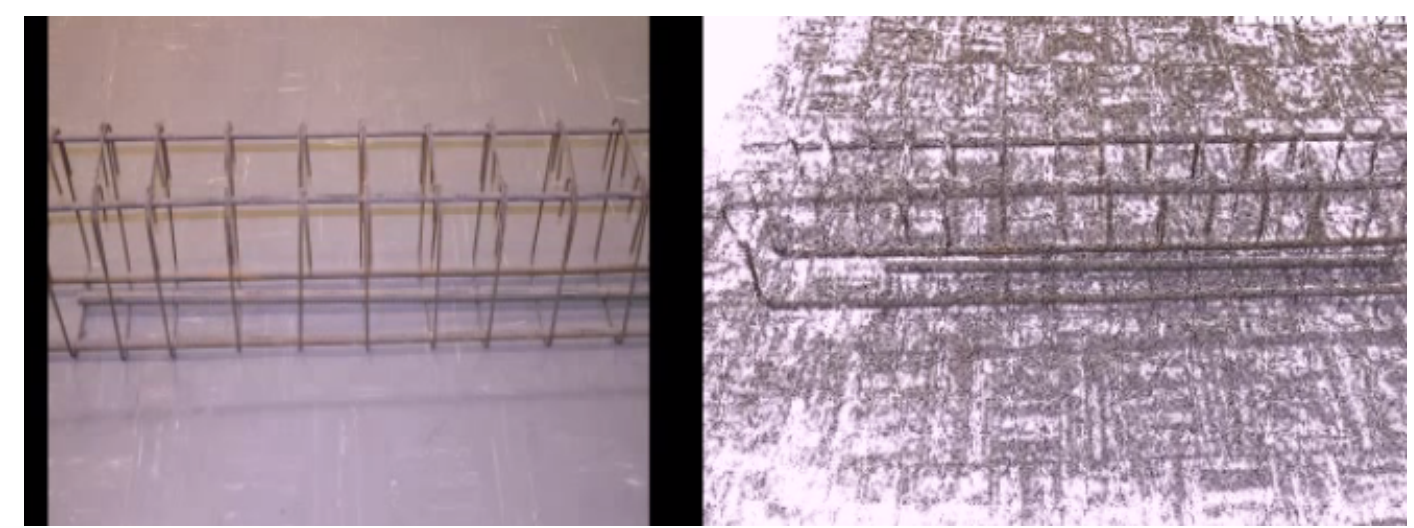

Figure 4.5: Photograph (left) and point cloud model (right) using digital camera 2

The point cloud model is generated from the matching points between the photographs. After the point cloud model had been created, the model was meshed to generate a shaded surface. From the shaded surface a textured model was generated which is used for dimensioning. Figure 4.6 shows the shaded and textured models. 

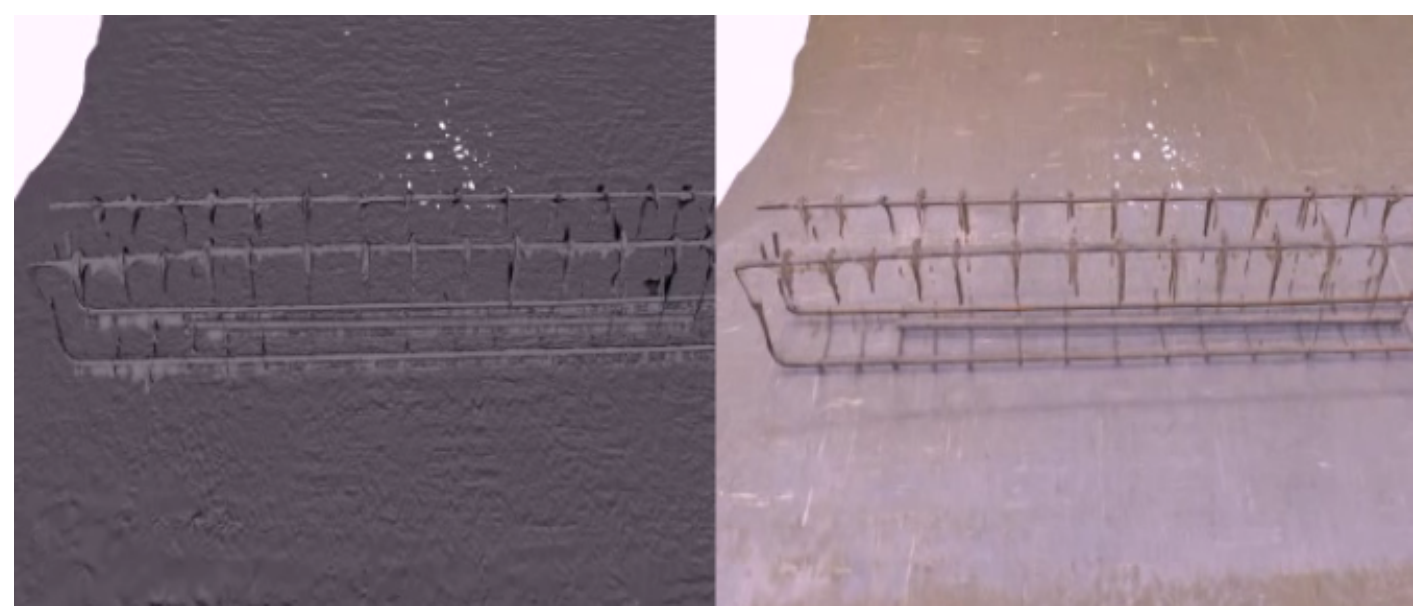

Figure 4.6: Shaded surface (left) and textured model (right) using digital camera 2

Table 4.8 shows the results of dimensions that were measured on the reinforcing beam and compared to dimensions on the 3D model generated through digital camera 2. The results were accurate and within the tolerance requirements specified by SANS 2001-CC1 (2007) (see Table 4.2). Dimensions of the reinforcement diameter, stirrup spacing and reinforcement spacing were used in the experiment.

Table 4.8: Dimensions measured when generating a point cloud model from camera 2 images.

\begin{tabular}{cccc}
\hline Dimension & $\begin{array}{c}\text { Software } \\
\text { measured } \\
\text { dimension } \\
(\mathbf{m m})\end{array}$ & $\begin{array}{c}\text { True } \\
\text { dimension } \\
(\mathbf{m m})\end{array}$ & $\begin{array}{c}\text { Percentage } \\
\text { deviation (\%) }\end{array}$ \\
\hline 1 & 137.4 & 135.0 & 1.8 \\
\hline 2 & 201.0 & 200.0 & 0.5 \\
\hline 3 & 160.1 & 160.0 & 0.1 \\
\hline 4 & 138.1 & 138.0 & 0.1 \\
\hline 5 & 17.1 & 16.0 & 6.9 \\
\hline 6 & 318.0 & 315.0 & 1.0 \\
\hline
\end{tabular}

Most of the dimensions are within 2\% accurate, except for the measurement of the bar diameter. The bar diameter is too small to obtain an accurate dimension. Furthermore, the model generates a little electronic noise on the surface of the object. The noise decreases the visibility of the object and makes it difficult to measure small dimensions accurately. To obtain results that are more accurate and a model with less noise, a better camera should be used. If the bar diameter dimension is not required, digital camera 2 possesses the minimum technical requirements to produce an accurate model. Figure 4.7 shows the 3D model (after processing shown in Figures 4.5 and 4.6) that was generated from using camera 2 . 


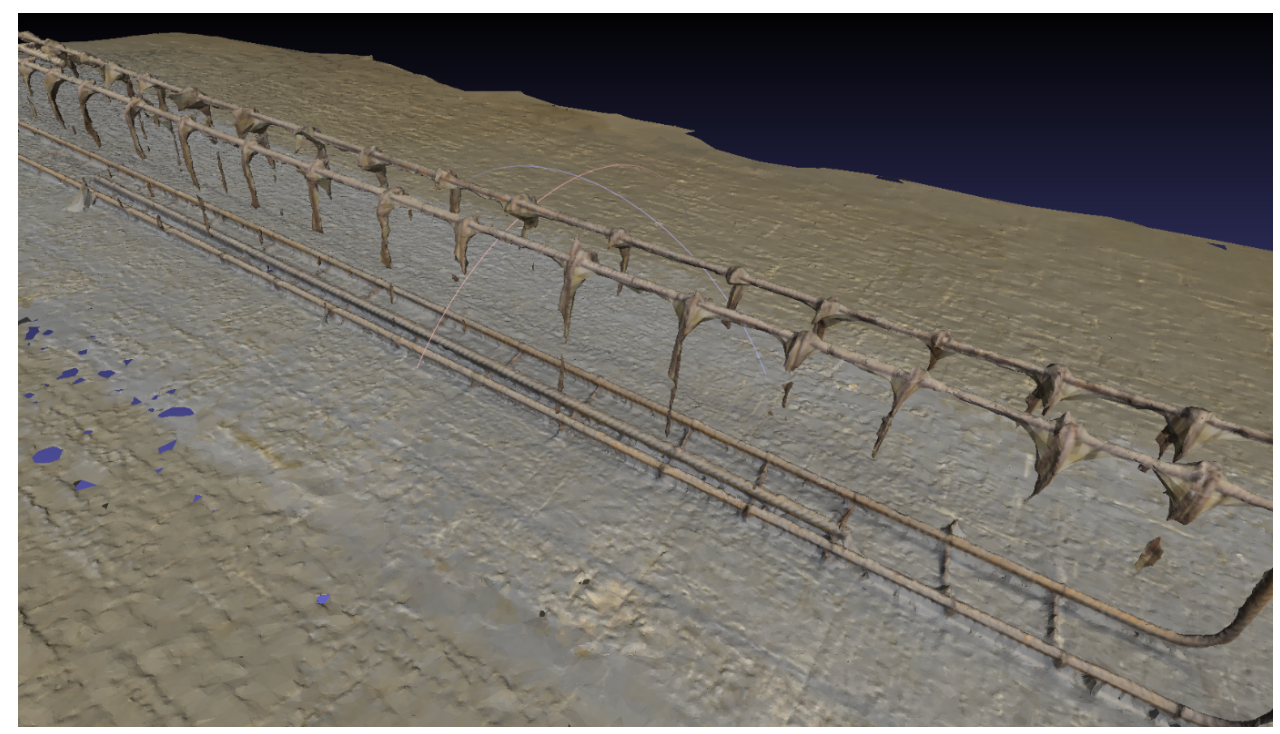

Figure 4.7: Final meshed model using digital camera 2

The ease of use and practicality of this system was subsequently evaluated. The primary disadvantage with the use of the software was that the computer processing time used to generate the model was 1.5 hours. A computer with 8 GB of Random Access Memory (RAM) and a graphics card with 4096 Megabytes of dedicated video memory was used to generate the model. When working on a remote site it is required that the images are downloaded from the camera and uploaded to a central cloud storage system so that the remotely situated stakeholders can access it. The data generated by the model occupied more or less 1 Gigabyte (GB) of hard disk memory.

Table 4.5 on $\mathrm{p} 69$ shows the evaluation criteria that was used for the system. Table 4.9 shows the results from the assessment with assigned scores by the author.

Table 4.9: Evaluation of the pointcloud software after tests in the laboratory

\begin{tabular}{lcl}
\hline Criteria & Score out of $\mathbf{5}$ & Comment \\
\hline Ease of use & 3 & Difficult to get used to software. \\
\hline Accuracy & 3 & Accurate to more or less $2 \%$. \\
\hline $\begin{array}{l}\text { Functionality } \\
\text { Data usage and } \\
\text { computer requirements }\end{array}$ & 3 & $\begin{array}{l}\text { Program can function only under } \\
\text { certain conditions; failure occurs to } \\
\text { monitor important aspects on site. }\end{array}$ \\
\hline $\begin{array}{l}\text { Time usage for complete } \\
\text { monitoring }\end{array}$ & 2 & $\begin{array}{l}\text { Internet will be required to upload } \\
\text { data onto a cloud server. Requires } \\
\text { a good computer. }\end{array}$ \\
\hline
\end{tabular}

The information in Table 4.9 shows that the software was not easy to use. The accuracy of all of the measurements, except for bar diameter, was very good and within $2 \%$ of the true dimension. The functionality of the software and monitoring process was fair. The model generated is not entirely suitable to monitor dimensions on complex reinforcing structures. It would be difficult to generate a well-detailed model of a complex structure 
since photographs of the whole structure is required. The software is better suited for elements such as beams, slabs and columns. The total time required to use this monitoring technique is too long for it to be used effectively during construction.

Another software program, with similar features, is less time consuming. This software was also used to assess the performance of the technology system. Ten tests were conducted and the average computation time was more or less 45 minutes. The problem identified with this software was that the detail included in the model was much less compared to the other software. Therefore it was concluded that the level of detail decreases together with the computation time. Figure 4.8 shows the model generated from one of the ten tests with the alternative software. The additional nine tests that were conducted with the alternative software is not shown since all of them showed a lack of detail.

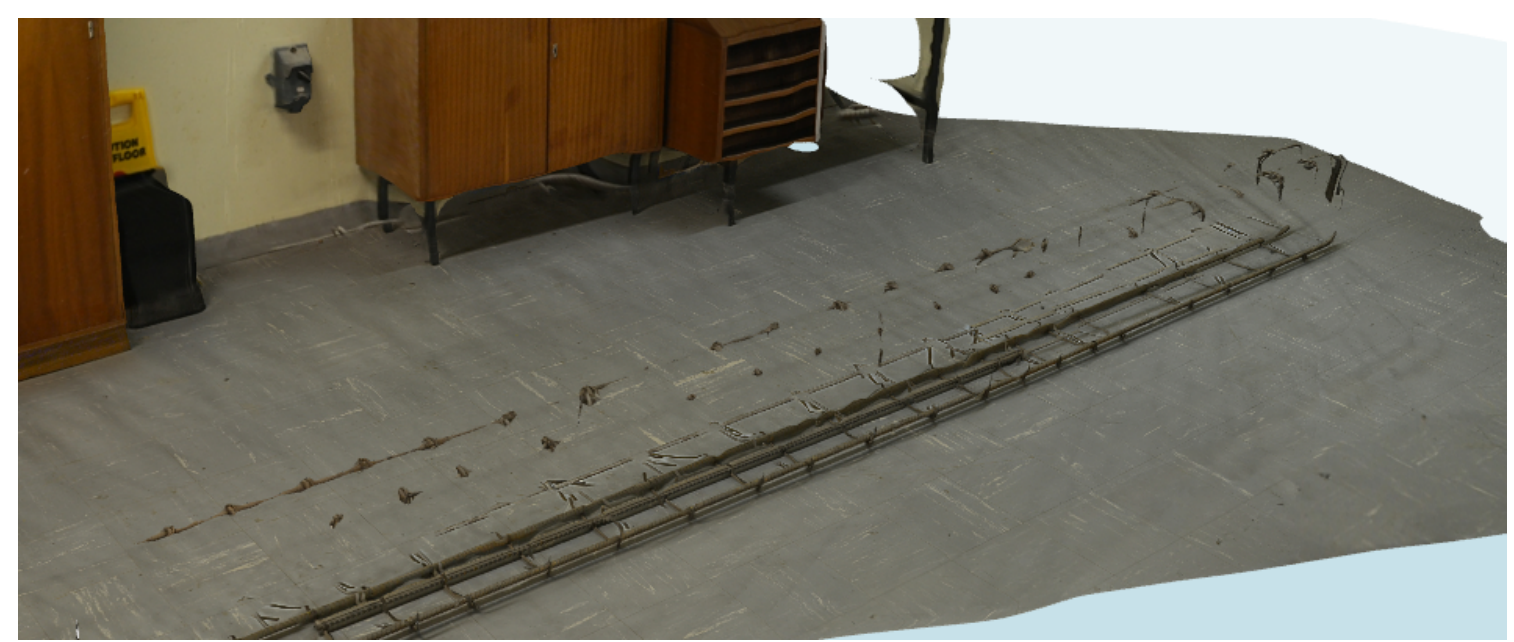

Figure 4.8: Point cloud model generated with software that requires less computation time.

The primary barrier identified with the use of point cloud modelling technology was the long computation time required to generate a detailed model. The conclusion was made that the technology would not be effective to use during construction. As technology improves in the near future, this could be a feasible technological solution to improve quality control. Golparvar-fard et al. (2011) mentioned that additional research is required to develop algorithms that automatically extract conventional or parametric CAD objects. Algorithms also need to be developed that optimise the computational cost and increase the quality of the models.

\subsubsection{Mobile software used to measure dimensions on photos}

Software that allows the user to take measurements on a photograph was assessed by the author. The evaluation form shown in Table 4.3 on $\mathrm{p} 66$ was completed by the author and is shown in Appendix B. Tests were conducted in the engineering laboratory at SU to determine the ease of use and the accuracy of the software.

As identified from Appendix $B$, the software is useful to assist in the monitoring of the following construction activities: 
- Steel spacing

- Slump test

- Lap-lengths

- Level of pull-out bars

- Position of HD bolts.

The software was tested on the reinforcement cage of a beam. For the software to provide accurate readings it requires calibration. When capturing the photograph, a rectangle of known dimensions is placed in the same plane as the dimensions that are measured. The locations measured for the experiment are shown in Figure 4.9 .

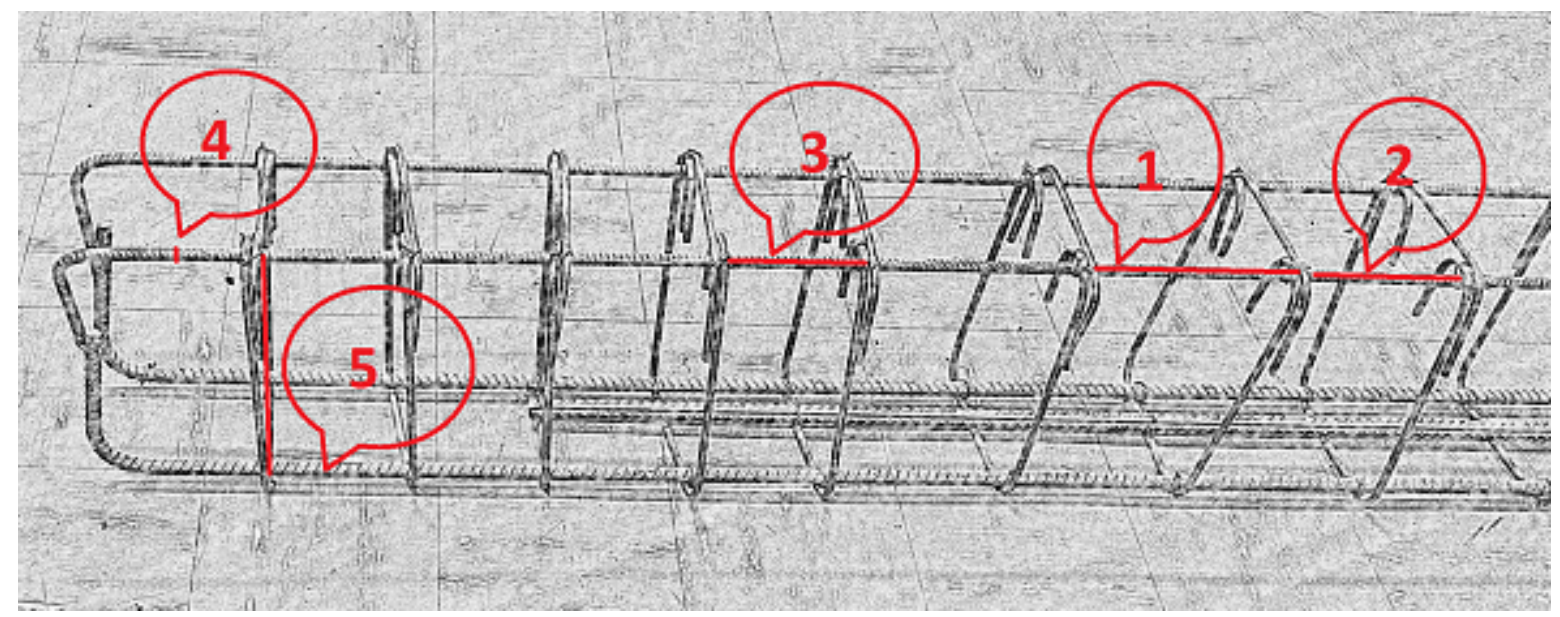

Figure 4.9: Locations measured on the reinforced beam-cage

Each of the photos were captured at a uniform distance from the beam-cage. Furthermore, the photos were taken at various angles to the vertical axis. Different angles were used to assess the accuracy as the angle varies from which the photograph is taken. It was assumed that during construction the user could capture the photo in any manner; therefore, it is important to assess the accuracy of the software under various conditions. In Figure 4.10, on a vertical plane, the photo is captured from point p2 and the object being captured in the photograph is at $\mathrm{p} 1$. The angle, theta $(\theta)$, represents the various angles that were used. 


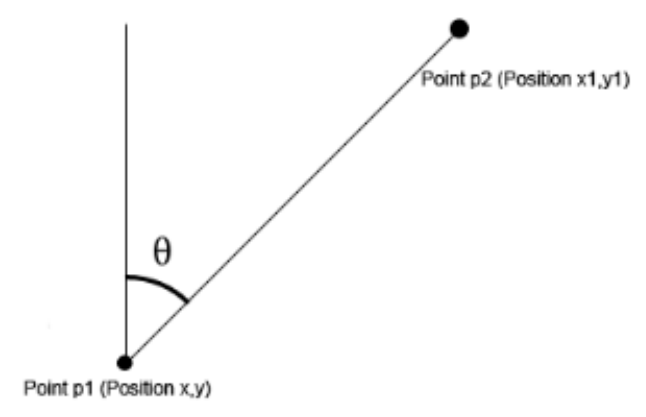

Figure 4.10: Illustration (in the vertical plane) of the vertical angle from which photos were taken.

The application software was tested by determining the accuracy of the dimensions for the stirrup spacing, reinforcement spacing and bar diameter. The accuracy of the software was determined by comparing the measured dimensions with the true dimensions of the reinforcement cage. The photographs were taken at the following angles with the vertical axis:

- $0^{\circ}$

- $30^{\circ}$

- $45^{\circ}$

- $60^{\circ}$.

Images were also captured at $90^{\circ}$ and $45^{\circ}$ with the horizontal axis to obtain dimensions of the side of the beam. Viewing Figure 4.11 in a horizontal plane, point p2 represents the position of the camera and p1 the object being captured.

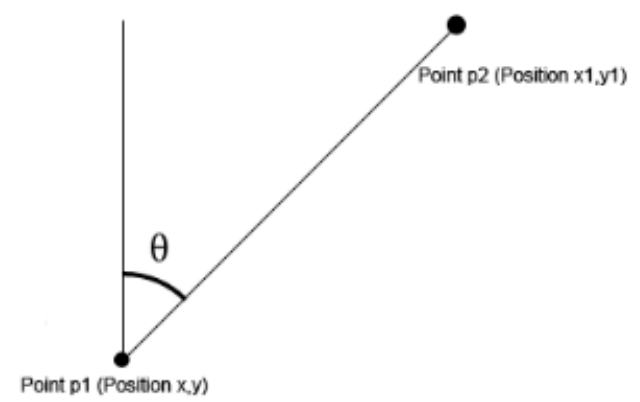

Figure 4.11: Illustration (horizontal plane) of the horizontal angle from which photos were taken. 
The test results showed that the software provided accurate dimensions for most of the measurements made. Table 4.10 shows the deviation percentages for the software dimension and the true dimension. The deviation percentages were low, except for when measuring the bar diameters. The deviations obtained for the bar diameter reached a maximum of $10 \%$.

No clear relationship was observed between the accuracy of the dimensions and the angle at which an image was captured. However, the accuracy decreased when dimensions were measured further away from the calibration reference. Table 4.10 shows the results that were obtained for the various tests.

Table 4.10: Test results for the image dimensioning software through use of a mobile phone.

\begin{tabular}{|c|c|c|c|}
\hline $\begin{array}{l}\text { Dimension } \\
\text { location }\end{array}$ & $\begin{array}{c}\text { Measured } \\
\text { distance }(\mathrm{mm})\end{array}$ & $\begin{array}{c}\text { True } \\
\text { Dimension } \\
(\mathrm{mm})\end{array}$ & $\begin{array}{c}\text { Percentage } \\
\text { deviation (\%) }\end{array}$ \\
\hline \multicolumn{4}{|c|}{0 degrees with vertical } \\
\hline 1 & 199.1 & 200.0 & 0.5 \\
\hline 2 & 159.0 & 160.0 & 0.6 \\
\hline 3 & 137.8 & 138.0 & 0.1 \\
\hline 4 & 12.6 & 12.0 & 5.0 \\
\hline \multicolumn{4}{|c|}{30 degrees with vertical } \\
\hline 1 & 199.2 & 200.0 & 0.4 \\
\hline 2 & 160.8 & 160.0 & 0.5 \\
\hline 3 & 140.3 & 138.0 & 1.7 \\
\hline 4 & 13.2 & 12.0 & 10.0 \\
\hline \multicolumn{4}{|c|}{45 degrees with vertical } \\
\hline 1 & 198.6 & 200.0 & 0.7 \\
\hline 2 & 149.9 & 160.0 & 6.3 \\
\hline 3 & 138.4 & 138.0 & 0.3 \\
\hline 4 & 12.7 & 12.0 & 5.8 \\
\hline \multicolumn{4}{|c|}{60 degrees with vertical } \\
\hline 1 & 200.3 & 200.0 & 0.2 \\
\hline 2 & 166.1 & 160.0 & 3.8 \\
\hline 3 & 134.3 & 138.0 & 2.7 \\
\hline 4 & 12.0 & 12.0 & 0.0 \\
\hline \multicolumn{4}{|c|}{ Side of beam with image taken orthogonally } \\
\hline 5 & 319.0 & 315.0 & 1.3 \\
\hline \multicolumn{4}{|c|}{ Side of beam with image taken at a 45 -degree angle } \\
\hline 5 & 317.0 & 315.0 & 0.6 \\
\hline
\end{tabular}

The author evaluated the software was according to its ease of use, accuracy, functionality, data usage and operation time consumed.

The software application was easy to use and operate since it is user friendly and has a 
logical interface layout. Furthermore, the results of the tests were accurate. The functionality of the program was considered as average since it was troublesome to include the calibration reference in each photo. Therefore, the process of obtaining images for the software can become difficult for complex structures. This decreases the functionality.

Such an application is useful to measure the rebar spacing and the concrete cover. Furthermore, it is useful to measure slump and the lap-lengths of the rebar. The photos can easily be exported to logging software to maintain a record of the quality checks. Table 4.11 summarises the evaluation with assigned ratings by the author.

Table 4.11: Evaluation of the software that is used to take measurements on photos.

\begin{tabular}{|c|c|c|c|c|c|c|c|}
\hline Criteria & $\begin{array}{l}\text { Very poor } \\
(1 / 5)\end{array}$ & Poor $(2 / 5)$ & Fair $(3 / 5)$ & Good (4/5) & $\begin{array}{l}\text { Excellent } \\
(5 / 5)\end{array}$ & Rating & Comments \\
\hline Ease of use & $\begin{array}{l}\text { Very difficult; } \\
\text { complex. }\end{array}$ & $\begin{array}{l}\text { Very difficult; } \\
\text { requires } \\
\text { training. }\end{array}$ & $\begin{array}{l}\text { Difficult; gets } \\
\text { better with } \\
\text { training. }\end{array}$ & $\begin{array}{l}\text { Easy to use; } \\
\text { interface layout } \\
\text { is logical. }\end{array}$ & $\begin{array}{l}\text { Simple user } \\
\text { interface; easy } \\
\text { to operate. }\end{array}$ & 3 & $\begin{array}{l}\text { Program is } \\
\text { user-friendly } \\
\text { and provides } \\
\text { helpful tips } \\
\text { during use, but } \\
\text { calibration is } \\
\text { troublesome. }\end{array}$ \\
\hline Accuracy & $<4 \%$ & $<3 \%$ & $<2 \%$ & $<1 \%$ & $<0.5 \%$ & 3 & $\begin{array}{l}\text { Measurements } \\
\text { accurate } \\
\text { within } 2 \% \text { for } \\
\text { most } \\
\text { measurements. }\end{array}$ \\
\hline Functionality & $\begin{array}{l}\text { Not suited for } \\
\text { intended use. }\end{array}$ & $\begin{array}{l}\text { Can be used } \\
\text { for intended } \\
\text { purpose; not } \\
\text { preferred. }\end{array}$ & $\begin{array}{l}\text { Usable for } \\
\text { intended } \\
\text { purpose; not } \\
\text { simple enough } \\
\text { to use. }\end{array}$ & $\begin{array}{l}\text { Good for } \\
\text { intended } \\
\text { purpose; most } \\
\text { aspects } \\
\text { justified. }\end{array}$ & $\begin{array}{l}\text { Program very } \\
\text { suitable for } \\
\text { intended } \\
\text { purpose; all } \\
\text { aspects } \\
\text { justified. }\end{array}$ & 3 & $\begin{array}{l}\text { Program can } \\
\text { determine } \\
\text { dimensions } \\
\text { only under } \\
\text { certain } \\
\text { conditions; will } \\
\text { not be able to } \\
\text { determine all } \\
\text { necessary } \\
\text { specifications } \\
\text { for inspection. }\end{array}$ \\
\hline $\begin{array}{l}\text { Data usage } \\
\text { and computer } \\
\text { requirements }\end{array}$ & $\begin{array}{l}\text { Large data files } \\
\text { to process; } \\
\text { requires fast } \\
\text { internet. }\end{array}$ & $\begin{array}{l}\text { Medium sized } \\
\text { data files; } \\
\text { requires fast } \\
\text { internet. }\end{array}$ & $\begin{array}{l}\text { Data files are } \\
\text { small and easy } \\
\text { to transfer; } \\
\text { requires } \\
\text { internet. }\end{array}$ & $\begin{array}{l}\text { Data } \\
\text { processing does } \\
\text { not require } \\
\text { internet; still } \\
\text { need high } \\
\text { computation } \\
\text { power. }\end{array}$ & $\begin{array}{l}\text { Program } \\
\text { requires no } \\
\text { internet } \\
\text { connection. }\end{array}$ & 4 & $\begin{array}{l}\text { Program uses } \\
\text { no internet to } \\
\text { operate; files } \\
\text { generated use } \\
\text { little hard disk } \\
\text { space. }\end{array}$ \\
\hline $\begin{array}{l}\text { Time usage for } \\
\text { complete } \\
\text { monitoring } \\
\text { process }\end{array}$ & $\begin{array}{l}\text { More than } 1.5 \\
\text { hours }\end{array}$ & $\begin{array}{l}\text { More than } 1 \\
\text { hour }\end{array}$ & $\begin{array}{l}\text { More than } 45 \\
\text { minutes }\end{array}$ & $\begin{array}{l}\text { Less than } 45 \\
\text { minutes }\end{array}$ & $\begin{array}{l}\text { Less than } 20 \\
\text { minutes }\end{array}$ & 5 & $\begin{array}{l}\text { Program } \\
\text { processing } \\
\text { speed is fast. }\end{array}$ \\
\hline
\end{tabular}

\subsubsection{Field photo-log software}

Software was identified that is used to keep a daily photo-log of tasks and inspections on site. The software is useful to prioritize and log the activities monitored on site. Furthermore it simplifies situations where the project is situated at a great distance from the stakeholders. The logging of quality checks, with images and videos as proof, assists to maintain an organised project record which improves overall quality management. 
Using the field software on its own was not considered as a technology for reducing construction errors. However, the software is considered in conjunction with other systems, such as the IP camera and image dimensioning software. The photo-log software enables users to complete schedules of quality checks that require regular maintenance. For example, a maintenance schedule for curing would include the regular checking of the wrapping condition or applying water to concrete at recurring intervals.

Considering the aforementioned, the software was not assessed by means of an evaluation form (step 1) nor by tests in the laboratory (

\subsubsection{IP camera system}

In Table 3 of Appendix B, the capability of the Internet Protocol (IP) camera system was considered and reviewed from the technical information presented in Appendix D.

Physical tests were not conducted with the technology since the IP camera is not used to dimension objects, but only monitor processes. The IP camera is used in a lessons-learned approach where an expert monitors the construction processes and suggests improvements to the process. Chapter 5 provides more information on the lessons learned approach as well as how it improves the quality control during construction.

The most important attribute of the IP camera is its ability to record processes and display it in real-time with sufficient detail. The completion of Table 3 (Appendix B) shows that the use of an IP camera system potentially enables improved quality control when monitoring the following construction activities:

- Use of the correct formwork

- Vibration of concrete

- Actions engaged in to prevent grout loss

- Monitoring the power floating process

- Measures taken to prevent the formwork from kicking

- Cleanliness of the formwork, shutter boards and equipment.

- Curing process.

\subsubsection{Remote identification technologies}

The use of Radio Frequency Identification (RFID) and QR code systems improve the material-handling on site. By placing RFID tags or QR codes on reinforcement bundles, a supervisor can easily scan the tag/code to identify the correct location of it on site. These systems were not assessed in the same manner as the other technologies. Therefore, the evaluation does not follow the same structure as the other technologies identified. It is assumed that the RFID tags and QR codes are capable of functioning according to its intended purpose (scanning tags/codes to obtain or register information). 
The motivation for including the use of these systems was determined after receiving feedback comments during a site visit and reading a study by Elbeltagi (1987) that identified various problems that occur when considering the site layout. Elbeltagi (1987) identified that materials that arrive on site is unloaded at the incorrect locations. This impedes the smooth flow of work on site. The use of these systems are useful when the communication, administration and supervision on site are poor.

The use of RFID tags and QR code systems aid in the process of distributing the reinforcement to its correct location on site. During a site visit, it was noticed that all the reinforcing was delivered at a single location and then distributed to various locations on the construction site. This led to difficulties for the supervisor in finding the correct reinforcement for different parts of the construction works. This results in the use of incorrect reinforcement and causes delays in construction.

Ultimately, such a system improves the work flow to save time on site. It can prevent rework by ensuring that the correct reinforcement is used at each point of construction. If poor performance is expected from management then such a system can be useful. It is difficult to quantify the benefits of using these systems for the aforementioned purpose since little information was available of cases where it was used as such.

\subsubsection{Concrete curing sensors}

The concrete sensors were not evaluated. The sensors are only used during the curing of concrete. Therefore, it was reasoned that it is not necessary to complete an evaluation form that covers most of the construction activities. Furthermore, it is assumed that the concrete sensors are capable of functioning according to its intended purpose and that the accuracy of the device, as provided by the developers of the product, is correct.

The sensors provide information on the moisture condition and temperatures of the concrete during curing. Adequate curing is important to ensure that a durable and high strength concrete is obtained. Curing is important to increase the strength of the microstructure of concrete. General curing procedures do not enable the contractor to know what the immediate moisture condition of the concrete is.

\subsection{Summary of technology evaluation}

In this Chapter, important construction activities were identified from which errors regularly occur. Preventative measures were identified that are useful to reduce the construction errors.

An evaluation criteria was used to determine the capability of the technologies to monitor the various construction activities. The evaluation criteria assisted in identifying which technologies could be used to monitor certain construction activities.

The laser scanner, point cloud software and image dimensioning software were assessed more extensively through tests in the SU laboratory. The accuracy and time usage of these 
technologies are important to ensure that dimensions are measured accurately and that time consumption is not long while implementing the technology. It was found that these technologies adhere to the accuracy requirements provided in SANS 2001-CC1 (2007). The time consumption of the point cloud software was very long and was eliminated as a possible technology for quality monitoring.

Each of the remote identification- and the concrete sensor technologies were considered for one quality check. However, the other technologies were identified as being useful for various quality checks.

The information gathered in this Chapter is useful in Chapter 5 to establish how effective the technologies could be used prevent errors resulting from the identified construction activities. The experimental results in this Chapter is subsequently used to calculate the effectiveness of the laser scanner and image dimensioning software to reduce rework. 


\section{Chapter 5}

\section{Impact of technology on rework}

Figure 5.1 shows the scope of this Chapter compared to other Chapters in this study.

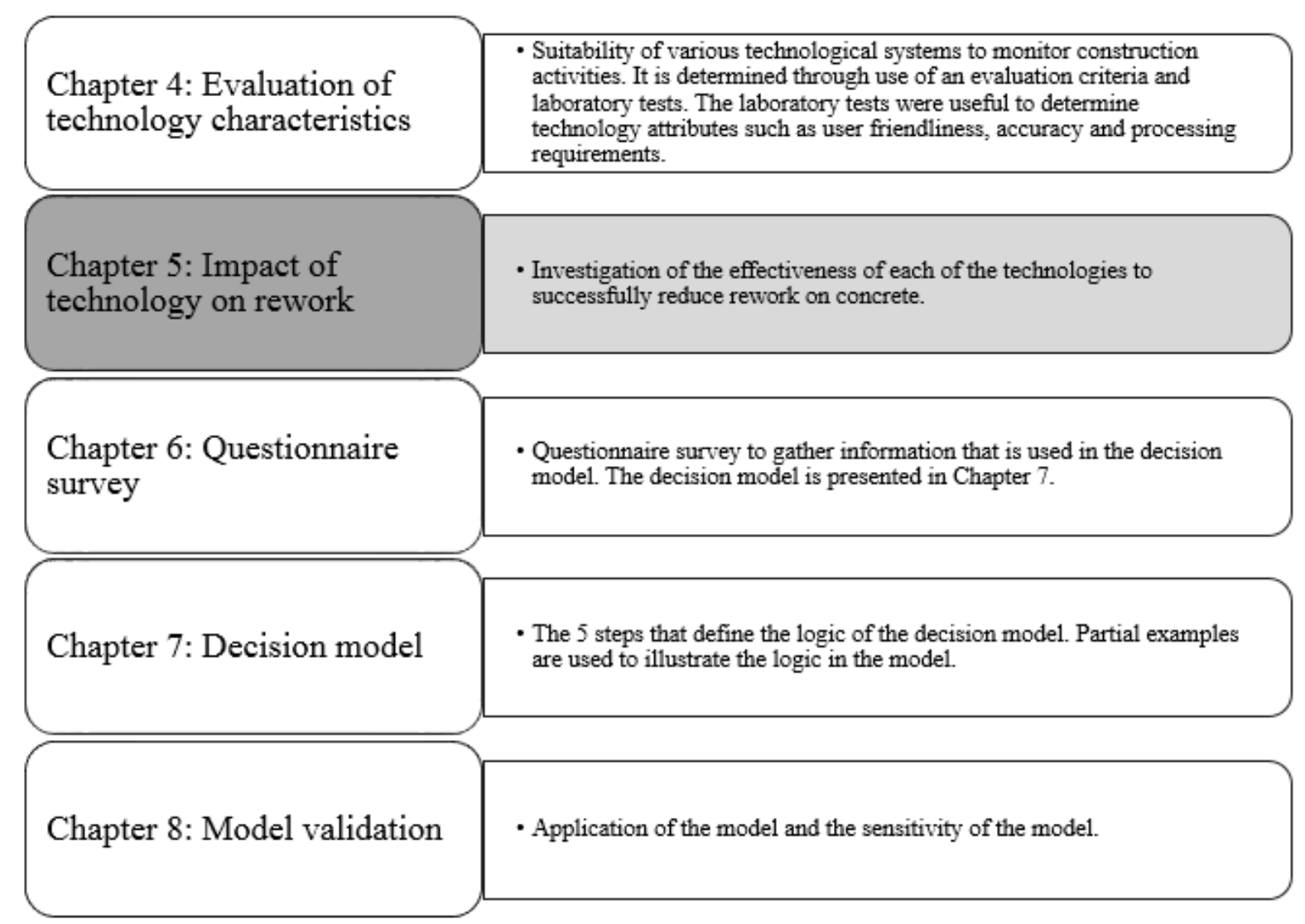

Figure 5.1: Scope of Chapter 5

In this Chapter, the effectiveness of the technological systems to monitor the identified construction activities is investigated. The effectiveness is determined since it is assumed that the technological systems are not 100 percent capable of monitoring the construction activities in such a manner that all the construction errors are prevented. The effectiveness is determined through use of histograms with data from previous case studies and laboratory test results (Chapter 4) as input. 
The concept of a lessons-learned approach and the effectiveness thereof is also described. The lessons-learned approach is used as a method to improve quality control. It was used where the effectiveness of technology could not be quantified easily and where the construction quality relies primarily on the construction process.

Each of the technological systems are considered separately with the construction activities that it can monitor. For each technological system the author states how it would be used to monitor the construction activity.

The effectiveness of each technological system, to reduce rework, is used in the technology investment model developed in Chapter 7 .

A diagram is presented in Figure 5.2 to show the content of this Chapter. For each of the technological systems an effectiveness is derived according to the its capability to successfully reduce construction errors. Figure 5.2 shows the input information and method used to derive the effectiveness for each technology system. Furthermore, it shows the construction errors that are reduced through use of the technology.

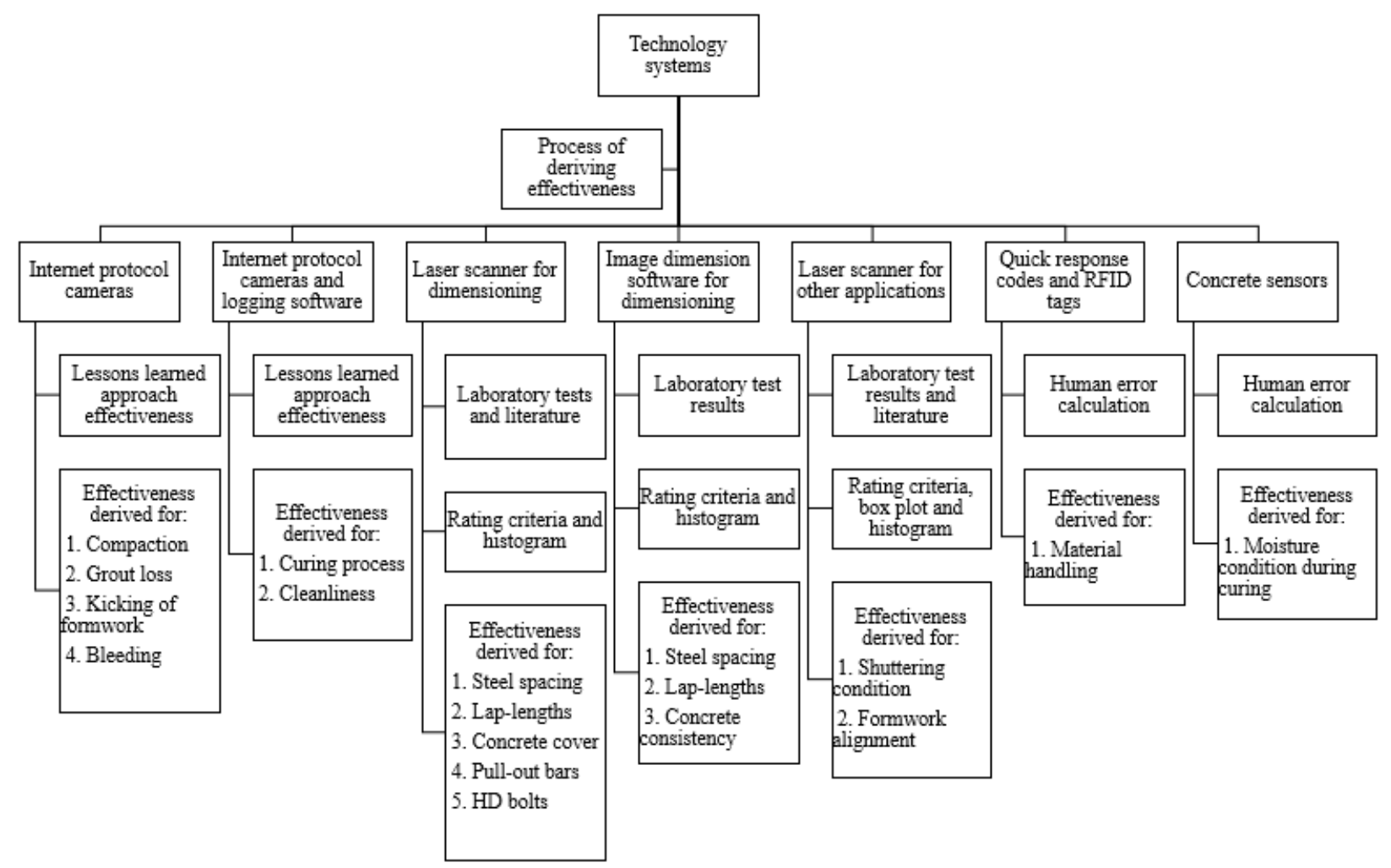

Figure 5.2: Structure of content in Chapter 5

\subsection{Risks to mitigate through use of technology}

The following risks were identified that could lead to rework during the construction of structural concrete works. These risks are considered for mitigation through use of technological systems. 
Risk: Incorrect steel spacing leads to rework.

Risk: Incorrect compaction of concrete leads to rework.

Risk: Occurrence of grout loss leads to rework.

Risk: Incorrect concrete cover leads to rework.

Risk: Kicking of formwork leads to rework.

Risk: Occurrence of bleeding leads to rework.

Risk: Use of concrete with incorrect consistency (slump) leads to rework.

Risk: Incorrect reinforcement distribution on site lead to incorrect bars being used and time delays.

Risk: Incorrect curing processes of concrete lead to rework.

Risk: Incorrect lap-lengths of reinforcement leads to rework.

Risk: Incorrect position of HD bolts lead to rework.

Risk: Pull-out bars at the incorrect level and location leads to rework.

Risk: Poor condition- and deformed shutter boards lead to rework.

Risk: Sub-standard formwork influences dimensions and alignment and lead to rework.

Risk: Poor maintenance of equipment lead to breakages and subsequently to delays during critical processes.

\subsection{Effectiveness of IP cameras}

It was identified that IP cameras, by means of a lessons learned approach, can be useful to monitor the following construction activities:

- Compaction

- Grout loss

- Kicking of formwork

- Bleeding.

Figure 5.3 shows the outline for determining the effectiveness of IP cameras to reduce the construction errors under consideration. 


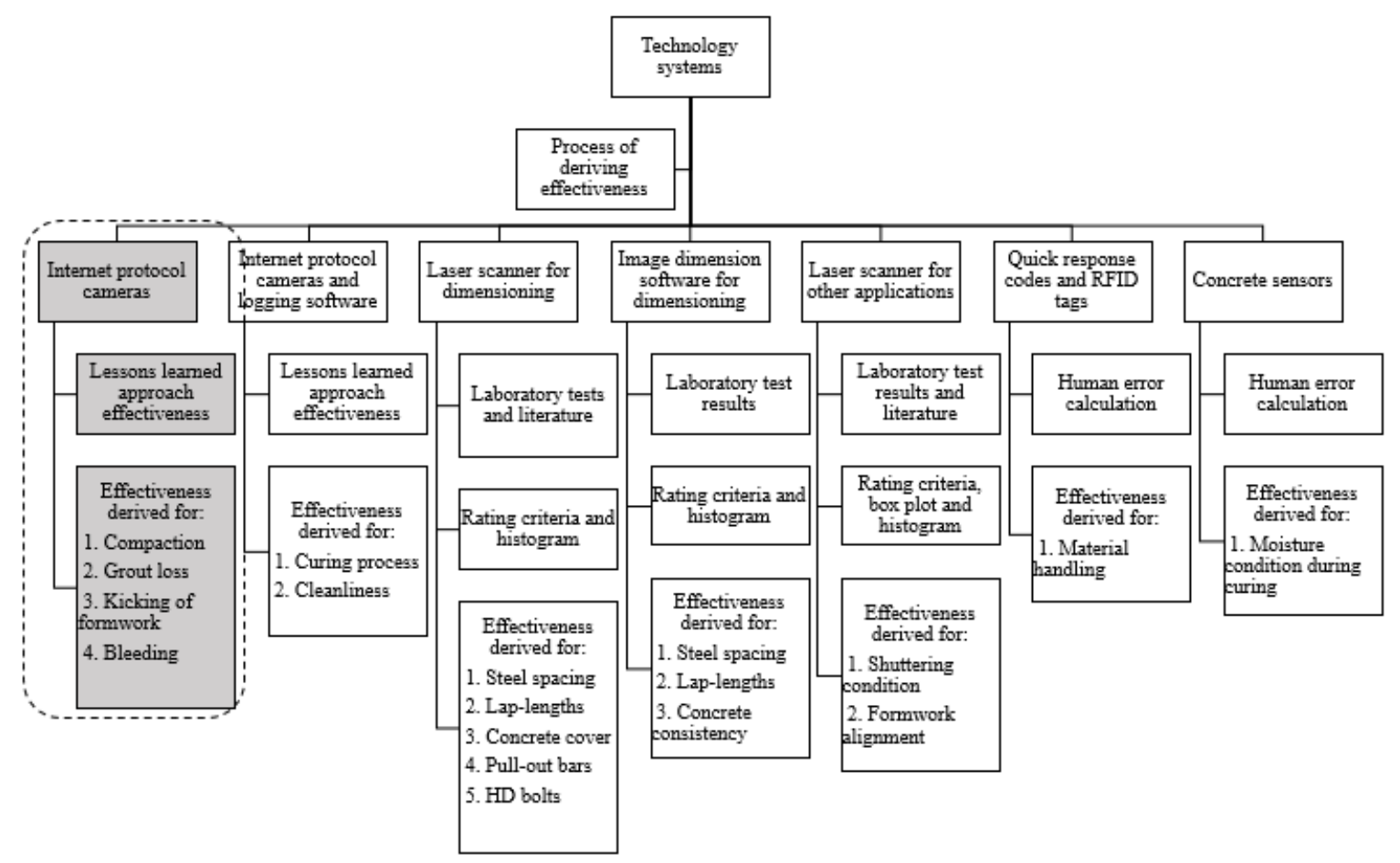

Figure 5.3: Process of deriving effectiveness of IP camera

\subsubsection{Using a lessons learned approach to improve quality con- trol}

Lessons learned is an effective way of transferring knowledge. Knowledge is shared regarding the elements that went according to plan and parts that could be improved, before moving to the next phase of a project. A lack of communication of crucial knowledge contribute to increased project cost, extended schedules and considerable rework (Jugdev, 2012).

The Project Management Body of Knowledge (PMBOK) defines lessons learned as "the learning gained from the process of performing the project. Lessons learned may be identified at any point. It is also considered a project record, to be included in the lessons learned knowledge base" (PMI, 2010).

It is said that lessons learned is quality improvement oriented and help correct process efficiency and effectiveness problems in a timely manner (Schindler, 2003). It helps deliver more successful projects, improve customer satisfaction (Kotnour, 1999) and helps participants learn about successful and unsuccessful practices (Busby, 1999). The Construction Industry Institute (2007) states that lessons learned elements are for the purpose of improving future performance.

Currently, lessons learned is applied from project to project. Technologies could provide the link between workers' point of activity and experts so that lessons learned are captured immediately as the project progresses. This provides the opportunity for real-time knowl- 
edge to be incorporated in future phases of the current project (Bowden, Dorr, Thorpe and Anumba, 2006). Survey results by Ferrada, Núñez, Neyem, Serpell and Sepúlveda (2016) showed that $81 \%$ of respondents trust that the use of mobile technology in the field would facilitate the use of a lessons learned system. Ferrada et al. (2016) developed a lessons learned system that was cloud based. Surveys showed that participants to the study provided a mean rating of $83 \%$ that such a system will improve process decision making. The participants also provided a mean rating of $77 \%$ that the system is suitable for use in everyday work.

A study in Dubai, by Yuan and Skaik (2008), used surveys to determine the benefits of introducing lessons learned. The respondents "strongly agreed" that introducing lessons learned would improve the following:

- Project cost, time and quality

- Minimize project risk

- Reduce variation orders

- Improve coordination

- Encourage project learning

- Increase company's competitive edge

- Enhance company's reputation.

However, in a study by Williams (2008) surveys showed that a lessons learned system helped only $67 \%$ of participants to identify clear issues. Moreover, the survey identified that only $55 \%$ of participants agreed that projects are more successful when using lessons learned.

A case study by Love et al. (2016) examined how rework can be reduced on a project in Australia. The study revealed that rework can be reduced by more than $50 \%$ by cultivating an error management system, focussing on continuous improvement and having an authentic leadership style in place.

The information obtained for lessons learned was used by the author to determine the effectiveness of an IP camera system to monitor quality. The cameras that were examined, show adequate technical capability to monitor the works. The effectiveness of the cameras were assumed from the lessons learned success rate in the study by Williams (2008).

It was reasoned that certain quality checks can be monitored by an expert with the assistance of an IP camera. The expert then follows a lessons learned approach by providing feedback to the workers on how to improve the processes during construction. Since only $55 \%$ of participants in the study by Williams (2008) agreed that a lessons learnt system works successfully, this was used as the effectiveness percentage for quality checks monitored by an IP camera in this study. 
The attribute requirements for a technological system, to assist in the lessons learned approach, was defined by the author and is shown in Table 5.1. From interviews, as discussed in Section 4.1, various factors were identified that should be monitored to prevent poor quality. These factors are discussed under each of the construction activities.

It is assumed that if the technology adheres to the requirements in Table 5.1, the technology should adequately monitor the details of the process to reduce rework. The effectiveness of the IP camera system, used in a lessons learned approach, is assumed as $55 \%$ for this study. This was derived from the study by Williams (2008).

Table 5.1: Requirements of technology to assist in a lessons learned approach

\begin{tabular}{ll}
\hline Requirements & Description \\
\hline & Excellent quality live video \\
Attributes to assist in lessons & feed; all necessary details seen; \\
learned approach & communication possible both \\
& ways \\
\hline
\end{tabular}

\subsubsection{Compaction}

Incorrect compaction leads to the poor quality of structural concrete. From the interviews it was identified that poor quality occurs due to over- or under compaction. During over compaction, segregation occurs and during under compaction, honeycombing occurs. To improve the quality control over the vibration process, a system is required that can monitor the following:

- Duration of compaction at each location

- Visibility of air bubbles as vibrator is slowly withdrawn from mix

- Quality of the concrete after compaction.

The referral to "all necessary details", in the attribute requirements of Table 5.1 , include the aforementioned points. An expert monitors the process through use of an IP camera. The expert can monitor the first occurrence of the compaction process and then provide suggestions to the team of how to improve the process.

\subsubsection{Grout loss}

Grout loss leads to poor concrete quality. A technological system, such as the IP camera, is required where the preparation of the formwork and the pouring process is monitored in real time. Through live streaming, the IP camera monitors the construction activity so that the required measures to prevent grout loss can be identified. The process requires monitoring from an experienced person.

When grout loss occurs, the expert changes the methodology to prevent grout loss from occurring on the work that follows. A change in methodology could include suggestions, such as the use of sealants and bolting the shutters. Table 5.1 shows the requirements for technological systems, such as the IP camera, to assist in the reduction of grout loss errors. 


\subsubsection{Kicking of formwork}

The kicking of formwork leads to the poor quality of structural concrete. It is prevented by bracing the shutters and reducing the pouring rate of concrete. Technology, such as the IP camera, can be used to monitor the processes of installing the bracing and pouring the concrete. By monitoring the processes, suggestions could be made to ensure that the process execution is improved the following time. During construction, these processes are repetitively executed. Quality control of the construction is improved by minimizing the mistakes through the lessons learned process.

Table 5.1 shows the technology attributes required for monitoring the kicking of formwork.

\subsubsection{Bleeding}

From the interviews (Chapter 4) conducted with industry professionals, it was found that bleeding occurs due to excessive power floating and vibration. The vibration of concrete has been covered in Section 5.2.2. An IP camera, through a lessons learned approach, can monitor the power floating process. A method change is suggested as soon as the power float process is incorrect. Table 5.1 shows the required technology capability to monitor construction activities that could lead to bleeding.

\subsubsection{IP camera system to monitor the identified processes}

An IP camera system has the capability to capture fine details and provides a manner of communication. There are IP cameras available that can rotate 360 degrees horizontally and that has a speaker through which the user can communicate to the staff on site. It is assumed that the available cameras provide adequate video quality to capture the required details. The use of an IP camera ensures that a lessons-learned technique is applied continually as the project progresses. It is not necessary for the expert to monitor each process as it is repeated, but rather to be involved at the start of construction for each type of element (slab, beam, column). Thus, errors are expected to reduce as the project progresses.

As decided under Section 5.2.1, an effectiveness value of 55\% was assumed for the use of IP cameras together with a lessons learned approach.

\subsection{Effectiveness of IP cameras with photo-log soft- ware}

The diagram in Figure 5.4 shows the content of this Section relative to the rest of the content in this Chapter. 


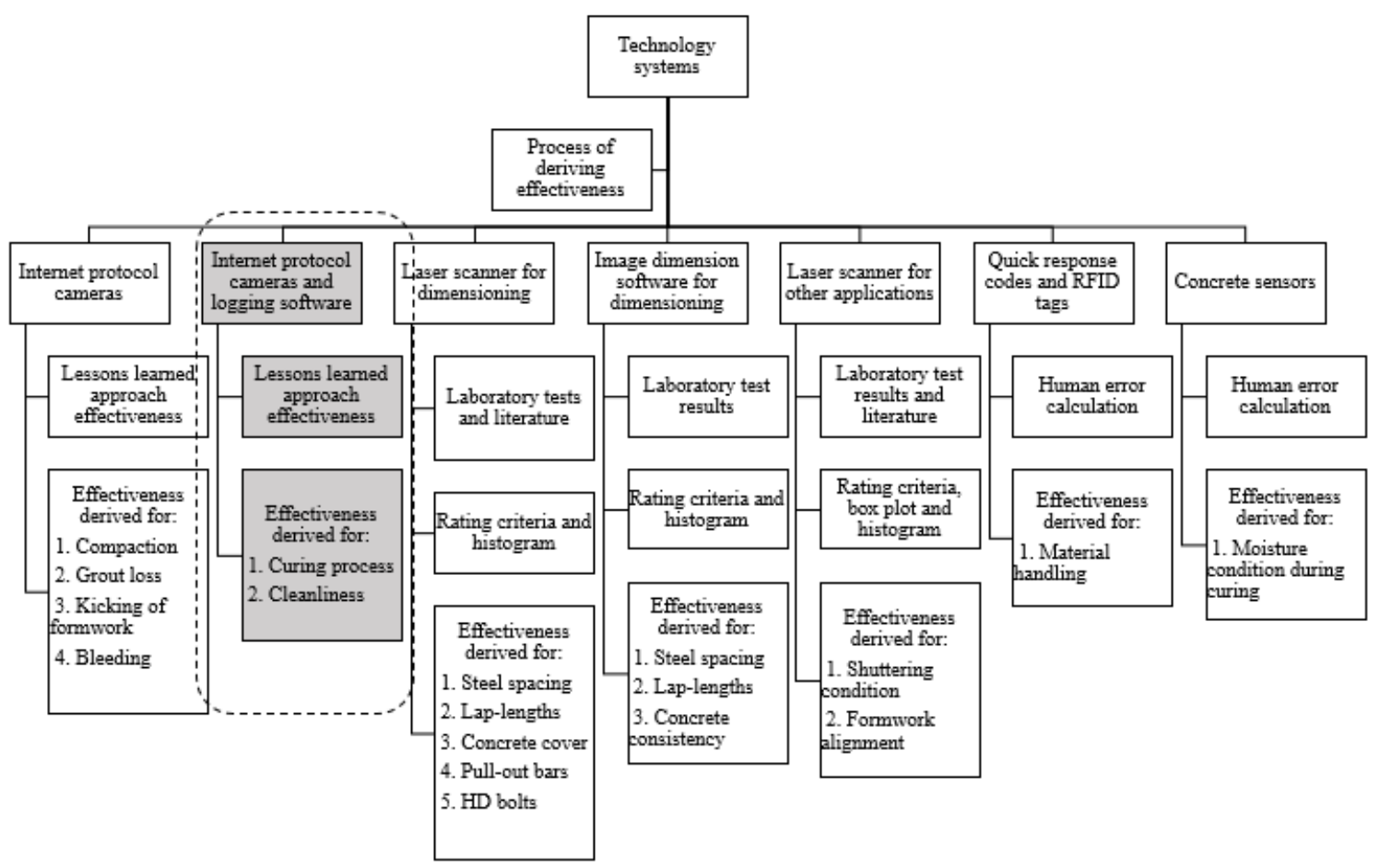

Figure 5.4: Content of this Section relative to other Sections

From interviews with industry experts, shown in Section 4.1, various factors were identified that should be monitored to prevent poor quality during construction. It was reasoned that IP cameras and logging software, through a lessons learned approach, can be useful to monitor the following construction activities:

- External curing processes

- Cleanliness of equipment and formwork.

The construction activities above require a technological system that can monitor the process and log the maintenance activities. The general requirements of a technological system to assist in the lessons learned approach are shown in Table 5.2. The term "necessary details" in Table 5.2 refers the specific details that should be monitored during the construction activities mentioned above.

Table 5.2: Technology attributes requirements to assist in a lessons learned approach

\begin{tabular}{ll}
\hline Requirements & Description \\
\hline & Excellent quality live video \\
Attributes to assist in lessons & feed; all necessary details seen; \\
learned approach & communication possible both \\
& ways; logging software to \\
& provide schedules \\
\hline
\end{tabular}

\subsubsection{External practices for the curing process}

Various problems regarding curing were identified in the interviews. The aspects of curing that require monitoring are as follows: 
- Ensure wrapping is applied

- If curing compound is used, ensure that it is applied to all the units and the entire surface area

- Monitor the maintenance of the curing process (ensure wrapping is continuously applied and undamaged).

Table 5.2 shows the technology attributes required when monitoring the curing process.

\subsubsection{Cleanliness of equipment and formwork}

The cleanliness state of equipment and formwork is difficult to monitor with technology. However, the processes engaged in to clean the equipment and formwork can be monitored through use of an IP camera. Furthermore, logging a schedule is useful to track the frequency of cleaning.

The quality check under consideration also includes the maintenance of equipment so that breakages are reduced during critical times in construction. The lessons learned approach, through use of an IP camera, is used to monitor the cleaning process and to suggest improvements to the methodology. Table 5.2 shows the attributes required from technology to monitor the cleanliness of equipment and formwork.

\subsubsection{Application of IP camera and field photo-log software}

An IP camera can be used to monitor the curing process. It is used to monitor the curing process so that a lessons learned approach is applied in the event that the expert is dissatisfied with the methodology of the process. Furthermore, the logging software keeps track of which elements have received the curing compound or wrapping. A maintenance schedule is important to ensure that the wrapping is regularly checked for damage.

The technological combination of an IP camera and logging software provides the necessary requirements to ensure that the cleanliness of equipment and formwork is monitored adequately. It is difficult to quantify the effectiveness of such a system for the construction activities under consideration; therefore, the effectiveness of the lessons learned approach was assumed. The effectiveness of the lessons learned approach was assumed from a study by Williams (2008) as 55\%. Thus, the assumed effectiveness of using an IP camera to prevent the identified construction errors is $55 \%$.

\subsection{Laser scanner and image dimensioning software for dimensioning checks}

Laser scanners and image dimensioning software (IDS) are useful for the monitoring of construction activities that require accurate dimensioning. It was identified that laser scanners and IDS can monitor the following construction activities:

- Laser scanner 
- Steel spacing

- Lap-lengths

- Concrete cover

- Pull-out bars at right level and correct location

- Position of holding down (HD) bolts

- Image dimensioning software

- Steel spacing

- Lap-lengths

The diagram in Figure 5.5 shows the content of this Section relative to the content of the other Sections in this Chapter.

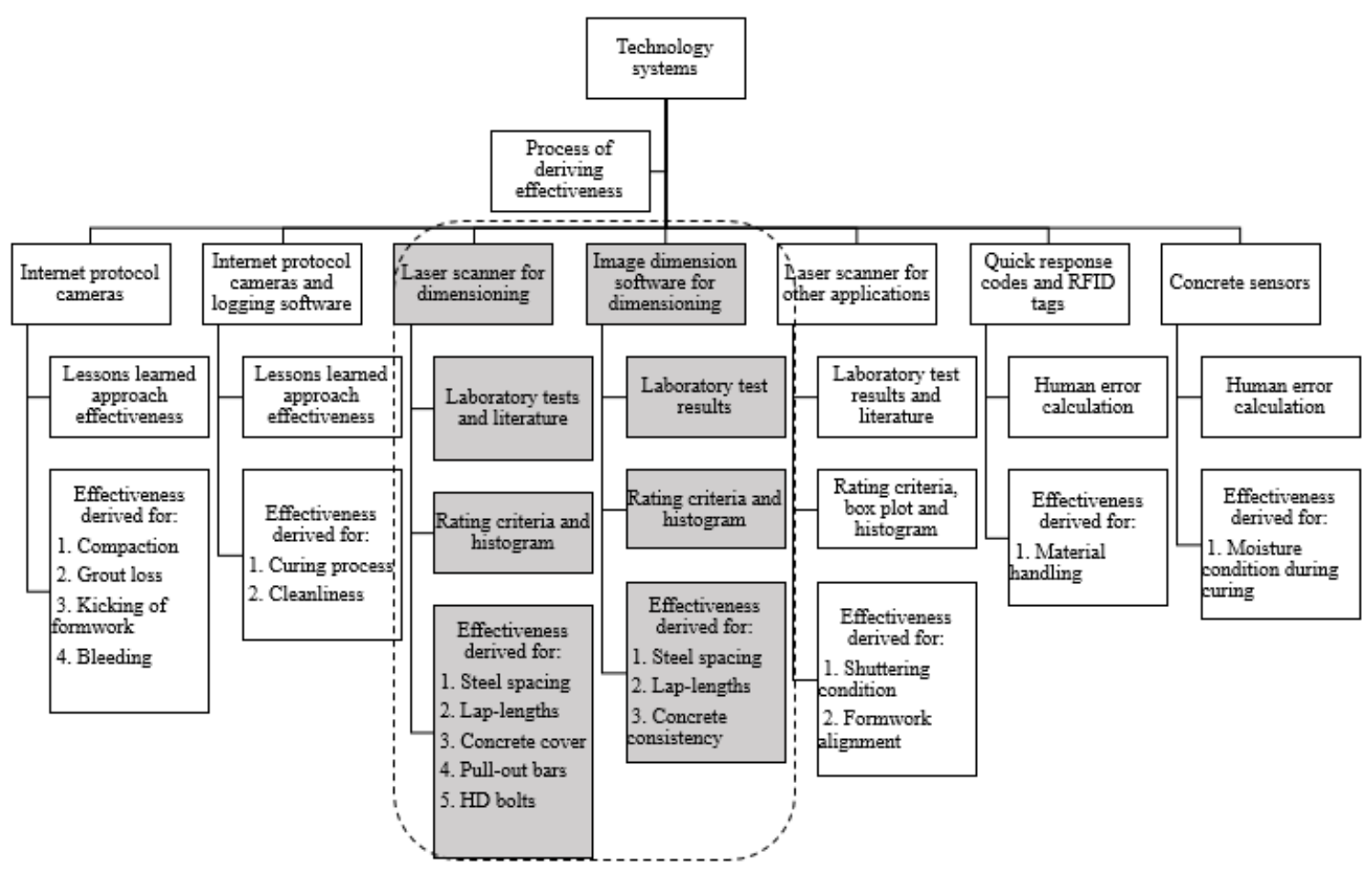

Figure 5.5: Content of this Section relative to other Sections in this Chapter

Table 5.3 shows the rating criteria established, by the author, for the monitoring of steel spacing and lap-lengths. The criteria was chosen specific to this study, but the approach can be different when determining the technology effectiveness for different scenarios. The author chose this approach so that the technology effectiveness is dependant on the accuracy and computation time of the technology. It was reasoned that dimensioning checks require high accuracy and should ideally be conducted with low computation time. Furthermore, recall that the aim of the study is to develop a decision model that assists in the investment decision of technology. Therefore, the scope was limited to develop a rating criteria that would perfectly represent conditions of technological effectiveness in field. 
Compared to the rating criteria established for concrete cover-, pull-out bar- and HD bolt checks; the steel spacing and lap-length rating criteria have less time allocated to conduct the check with technology. These are high frequency quality checks. Thus, if too much time is spent on monitoring the steel spacing and lap-lengths it would lead to excessive time being consumed during the monitoring process. Therefore, the effectiveness of the system is reduced if the technology computation time is long. Table 5.3 shows the criteria established for monitoring steel spacing and lap-lengths.

Table 5.3: Rating criteria defined for the monitoring of steel spacing and lap-lengths

\begin{tabular}{cl}
\hline Rating criteria & Description \\
\hline 1 & $\begin{array}{l}\text { Accuracy deviation }>3 \% \\
\text { and/or Processing time }>2 \\
\text { hours }\end{array}$ \\
\hline 2 & $\begin{array}{l}\text { Accuracy deviation }<3 \% \\
\text { and/or Processing time }<2 \\
\text { hour }\end{array}$ \\
\hline \multirow{2}{*}{3} & $\begin{array}{l}\text { Accuracy deviation }<2 \% \\
\text { and/or Processing time }<1 \\
\text { hour }\end{array}$ \\
\hline \multirow{2}{*}{4} & $\begin{array}{l}\text { Accuracy deviation }<1 \% \text { and } \\
\text { Processing time }<0.5 \text { hours }\end{array}$ \\
\hline & $\begin{array}{l}\text { Accuracy deviation }<0.5 \% \text { and } \\
\text { Processing time }<20 \text { minutes } \\
\text { and system captures all the } \\
\text { required detail }\end{array}$ \\
\hline
\end{tabular}

Table 5.4 shows the rating criteria established for technology to monitor concrete cover, pull-out bar level and location, and position of HD bolts. These checks have more time assigned in the rating criteria, as shown in Table 5.4. since they are less frequent checks compared to steel spacing and lap-lengths. These quality checks are important and were identified as holding points during construction. Thus, construction cannot continue if the concrete cover, pull-out bars and HD bolts have not been checked.

Table 5.4: Rating criteria defined for the monitoring of concrete cover, pull-out bars and position of HD bolts

\begin{tabular}{cl}
\hline Rating criteria & Description \\
\hline 1 & $\begin{array}{l}\text { Accuracy deviation }>3 \% \\
\text { and/or Processing time }>2 \\
\text { hours }\end{array}$ \\
\hline 2 & $\begin{array}{l}\text { Accuracy deviation }<3 \% \\
\text { and/or Processing time }<1.5 \\
\text { hours }\end{array}$ \\
\hline \multirow{2}{*}{3} & $\begin{array}{l}\text { Accuracy deviation }<2 \% \\
\text { and/or Processing time }<1 \\
\text { hour }\end{array}$ \\
\hline \multirow{2}{*}{4} & $\begin{array}{l}\text { Accuracy deviation }<1 \% \text { and } \\
\text { Processing time }<45 \text { minutes }\end{array}$ \\
\hline & $\begin{array}{l}\text { Accuracy deviation }<0.5 \% \text { and } \\
\text { Processing time }<30 \text { minutes } \\
\text { and system captures all the } \\
\text { required detail }\end{array}$ \\
\hline
\end{tabular}




\subsubsection{Effectiveness of laser scanner to monitor steel spacing and lap-lengths}

To successfully monitor steel spacing and lap-lengths, a laser scanner is required that could accurately measure dimensions with low computation time. The rating criteria for the lap-length quality check corresponds to that of the steel spacing quality check. The reason therefore is that the nature of the quality checks are similar. Table 5.3 shows the rating criteria.

The effectiveness of the laser scanner was determined from experimental results by Kim, Sohn and Chang (2014), Golparvar-fard et al. (2011) and the author (Chapter 4) in the laboratory at SU.

A study by Kim et al. (2014) determined the accuracy of a laser scanning system when measuring the dimensions of pre-cast units. The study by Kim et al. (2014) included 30 data measurements with accuracy deviations.

A study by Golparvar-fard et al. (2011) investigated the accuracy of point cloud models generated by laser scanners. Golparvar-fard et al. (2011) did the same experiments for generating point cloud models from photographs as shown in Chapter 4. Eight experiments were conducted. Four were of column dimensions and the other four of cuboidal masonry block dimensions. Of the four column tests, two were conducted on site and the other two in a laboratory. Of the four masonry block tests, two were conducted outside and the other two inside in a laboratory.

From the laser scanning demonstration presented at Stellenbosch University (2017), it was found that the duration of a single scan was 5 minutes. The processing time for a single scan was more or less 10 minutes (depending on the quality of the scan). A single scan would be adequate to determine dimensions on flat surfaces of reinforcement cages (slabs and one plane view of columns or beams). To determine dimensions on all the surfaces of a beam or a column cage, at least four scans are required. More than one scan would lead to excessive computation time and reduce the effectiveness of the technology.

\subsubsection{Developing the histogram for the laser scanner}

The histogram was developed for dimensioning on a single surface only (assume 2 scans are adequate to include enough detail for the top level of steel). The total processing time would be more or less 30 minutes, which prevents the scanner from receiving a rating that is higher than 4 out of 5 (Refer to Table 5.3).

The histogram was developed from 47 data points. Each of the accuracy deviations found in the study by Kim et al. (2014) and Golparvar-fard et al. (2011) as well as the laboratory test results were assigned a rating according to Table 5.3. The estimated probability of success, to reduce rework, was found by dividing the frequency of achieving a rating of 4 and higher by the total number of data points. See Equation (5.1).

$$
\text { Probability success } \%=\frac{\sum \text { Frequency } \geq 4}{\text { Total }}
$$




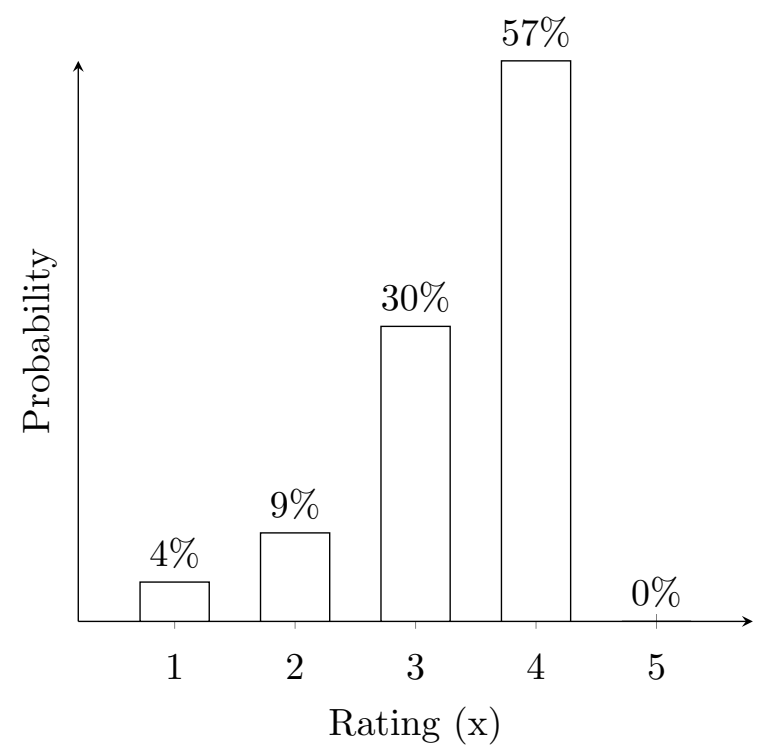

Figure 5.6: Histogram for laser scanner capability to monitor steel spacing and lap-lengths

The effectiveness of the laser scanner to successfully monitor steel spacing and lap-lengths is determined from the histogram. From the rating criteria in Table 5.3 it is suggested that the laser scanning system has a 4 out of 5 rating for it to work effectively on site. An assigned rating lower than 4 out of 5 would mean that the system takes longer than 30 minutes to complete the check and that the accuracy deviation is greater than $2 \%$.

From the histogram, shown in Figure 5.6, it was calculated that there is a 57\% probability of the laser scanner obtaining a rating of 4 or higher. Therefore, the effectiveness of the laser scanner to monitor steel spacing and lap-lengths is assumed to be $57 \%$.

\subsubsection{Image dimensioning software to monitor steel spacing and lap-lengths}

The same rating criteria (as obtained previously for the laser scanner) is used when considering the use of IDS for the monitoring of:

- Steel spacing

- Lap-lengths.

The requirements from technology to monitor steel spacing and lap-lengths are similar; therefore, the effectiveness value determined for each is the same.

In Chapter 4, experimental tests were conducted with the image dimensioning software. The rating criteria in Table 5.3 was used to assign ratings for the accuracy deviations determined from the experimental results in Chapter 4 (Table 4.10). Table 5.5 shows the accuracy deviations together with their assigned rating. The time consumed when using the software is less than 20 minutes. Therefore, a rating of 5 is assigned if the accuracy deviation is within $0.5 \%$. 
Table 5.5: Experimental values obtained from tests in the laboratory

\begin{tabular}{ccc}
\hline Data point & $\begin{array}{c}\text { Accuracy deviation } \\
\text { percentage }\end{array}$ & Rating \\
\hline 1 & 0.50 & 4 \\
\hline 2 & 0.60 & 4 \\
\hline 3 & 0.10 & 5 \\
\hline 4 & 0.40 & 5 \\
\hline 5 & 0.50 & 4 \\
\hline 6 & 1.70 & 3 \\
\hline 7 & 0.70 & 4 \\
\hline 8 & 6.30 & 1 \\
\hline 9 & 0.30 & 5 \\
\hline 10 & 0.20 & 5 \\
\hline 11 & 3.80 & 1 \\
\hline 12 & 2.70 & 2 \\
\hline 13 & 1.30 & 3 \\
\hline 14 & 0.60 & 4 \\
\hline
\end{tabular}

A histogram was developed for the assigned ratings in Table 5.5. The sample size consist of 14 different data measurements.

Figure 5.7 shows the histogram for the assigned ratings. For this study it is assumed that an assigned rating $\geq 4$ represents an occurrence where the technology successfully prevents the construction error. The frequency of the ratings that are $\geq 4$ make up $72 \%$ of the entire test sample. Therefore, the estimated effectiveness of the image dimensioning software to successfully reduce rework from incorrect steel spacing and lap-lengths is $72 \%$.

The image dimensioning software is not considered for the monitoring of pull-out bars since it requires the survey levels of the work to be checked. The software under consideration cannot link survey data.

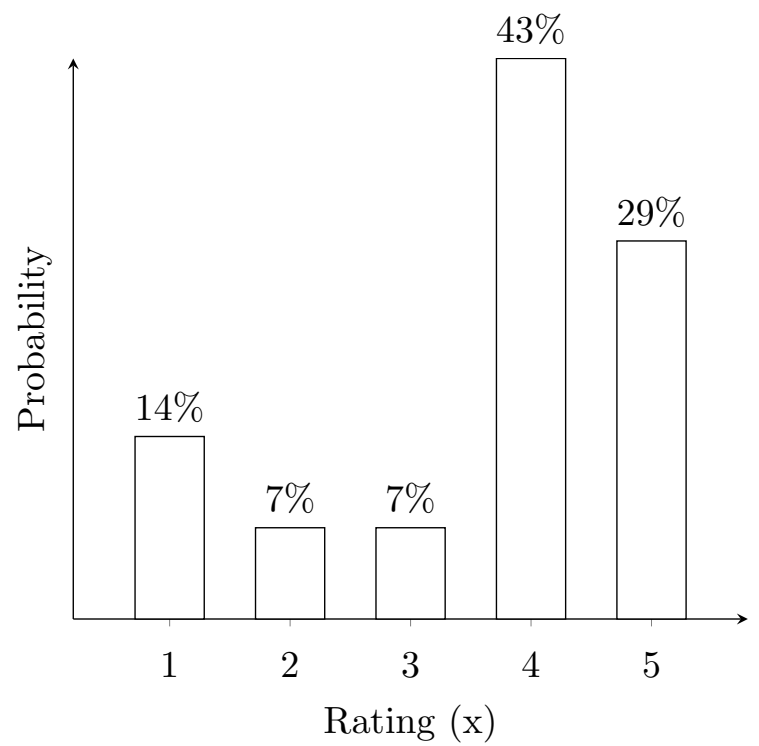

Figure 5.7: Histogram for IDS capability to monitor steel spacing and lap-lengths 


\subsubsection{Laser scanner to monitor concrete cover}

A laser scanner can be used to monitor the following with regards to concrete cover:

- Dimensions of cover blocks

- Count the number of cover blocks.

Incorrect concrete cover has a negative influence on the durability of structural concrete. Therefore, the quality of the concrete cover is a critical part in the construction process. The rating criteria established for a technological system to monitor concrete cover is shown in Table 5.4. Since the cover quality check is such a critical part in the construction process, additional time to conduct the check was added to the rating criteria.

A laser scanner can be used to scan the reinforcement cages and measure dimensions of cover blocks. Furthermore, the detailed model enables the counting of the number of blocks inserted. At least three scans are required to generate a model with enough detail for the cover quality check. The processing duration would be more or less 45 minutes. This prevents the system from obtaining an assigned rating that is greater than 4 out of 5. The same data points from the studies by Kim et al. (2014) and Golparvar-fard et al. (2011) as well as the experimental results were used to generate a histogram for the concrete cover check.

Figure 5.8 presents the histogram. It is assumed that the laser scanner should achieve a rating of at least 4 out of 5 to effectively prevent construction errors. The effectiveness of the laser scanner was estimated as $57 \%$ to monitor concrete cover in such a manner that rework is prevented.

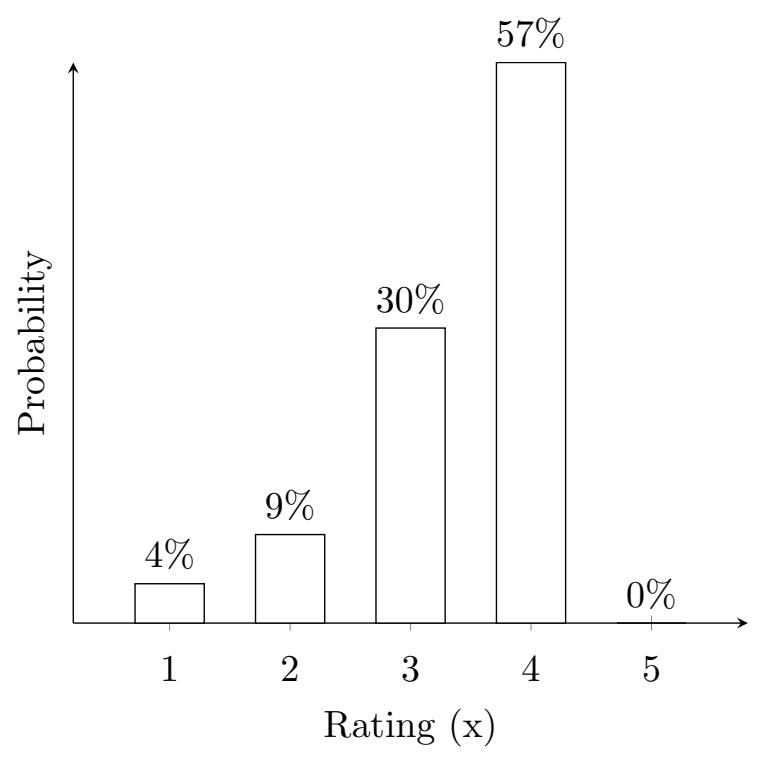

Figure 5.8: Histogram for laser scanner capability to monitor concrete cover 


\subsubsection{Laser scanner to monitor pull-out bars and HD bolts}

From the interviews it was found that problems occur when the levels of the pull-out bars are incorrect. Incorrect levels of pull-out bars lead to incorrect cover in the adjacent structural element. Currently, this is a holding point in construction and surveyors need to check the levels of the bars before the pouring of concrete commences. Since the correctness of pull-out bars was identified as a critical step in the quality monitoring process, the rating criteria was adjusted to allow more time for the monitoring process. The rating criteria is shown in Table 5.4 .

The positioning of holding down (HD) bolts is a less frequent, but important quality check. SANS 2001-CC1 (2007) specifies tolerances for HD bolts that are less than 3mm. The same rating criteria was used for the pull-out bar check and the HD bolt check. The quality checks required for HD bolt positioning are less frequent compared to e.g. steel spacing; therefore, more time was allocated to conduct the quality check. Table 5.4 shows the rating criteria used.

The histogram, in Figure 5.9, shows the assigned ratings for the laser scanner to monitor the pull-out bar and HD bolt quality checks. From the histogram, it is estimated that there is a $57 \%$ probability that the laser scanner would effectively prevent construction errors by monitoring the pull-out bars and the positioning of the HD bolts.

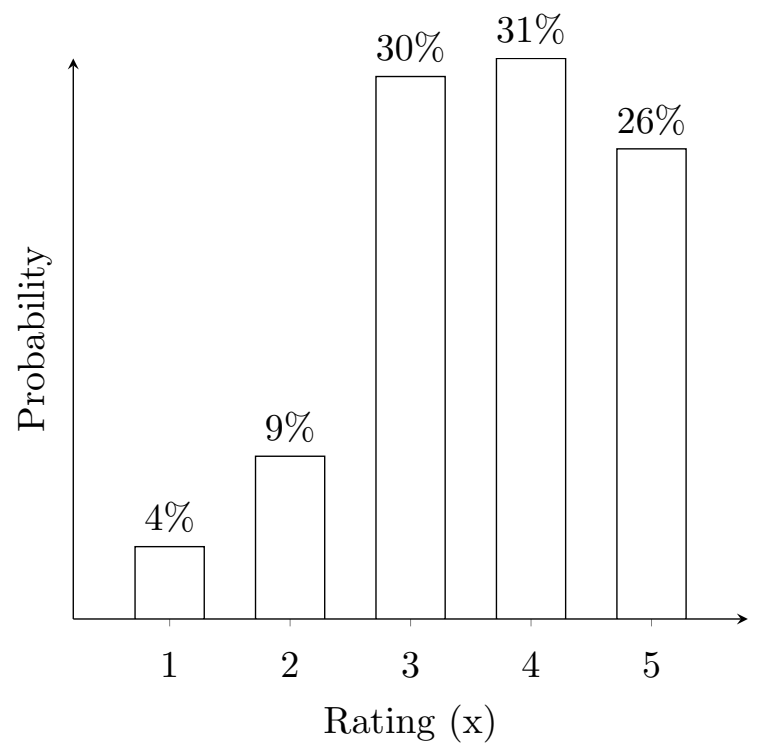

Figure 5.9: Histogram for the laser scanner system's effectiveness to monitor the pull-out bars and HD bolts

\subsubsection{Image dimensioning software with photo-log software to monitor concrete mix consistency}

The use of IDS in conjunction with logging software is proposed by the author to monitor the slump of concrete mixes. 
The consistency of the mix is monitored through the slump measurement of the concrete. A system is required that:

- Shows the slump being measured

- Logs data of the age of the concrete

- Shows the dimensions of the slump measurement.

Table 5.6 shows the rating criteria established by the author, for the requirements of technology to monitor the consistency of the mix.

Table 5.6: Rating criteria defined for the monitoring of concrete consistency

\begin{tabular}{|c|c|}
\hline Rating criteria & Description \\
\hline 1 & $\begin{array}{l}\text { Poor visual; Accuracy } \\
\text { deviation }>3 \%\end{array}$ \\
\hline 2 & $\begin{array}{l}\text { Poor visual; only footage } \\
\text { available to see testing of } \\
\text { slump; footage not available } \\
\text { early enough; Accuracy } \\
\text { deviation }<3 \%\end{array}$ \\
\hline 3 & $\begin{array}{l}\text { Video feed shows the testing of } \\
\text { slump; Accuracy deviation } \\
<2 \%\end{array}$ \\
\hline 4 & $\begin{array}{l}\text { Slump measurement visible on } \\
\text { image or video footage; age of } \\
\text { concrete known; Accuracy } \\
\text { deviation }<1 \%\end{array}$ \\
\hline 5 & $\begin{array}{l}\text { Excellent quality image or } \\
\text { video of slump test; exact age } \\
\text { known by linking delivery note } \\
\text { electronically; Accuracy } \\
\text { deviation }<0.5 \%\end{array}$ \\
\hline
\end{tabular}

The image dimensioning software (IDS) can be combined with logging software. The IDS is used to measure the slump and the image is then uploaded to the logging software. Furthermore, information regarding the age of the mix and the mix number is linked with the image in the logging software.

The accuracies specified in the rating criteria for the steel spacing and lap-lengths were similar; therefore, a similar histogram is obtained for the slump check. Thus, the same effectiveness value of $72 \%$ is estimated for the IDS to prevent construction errors from incorrect mix consistency. Figure 5.10 shows the histogram. 


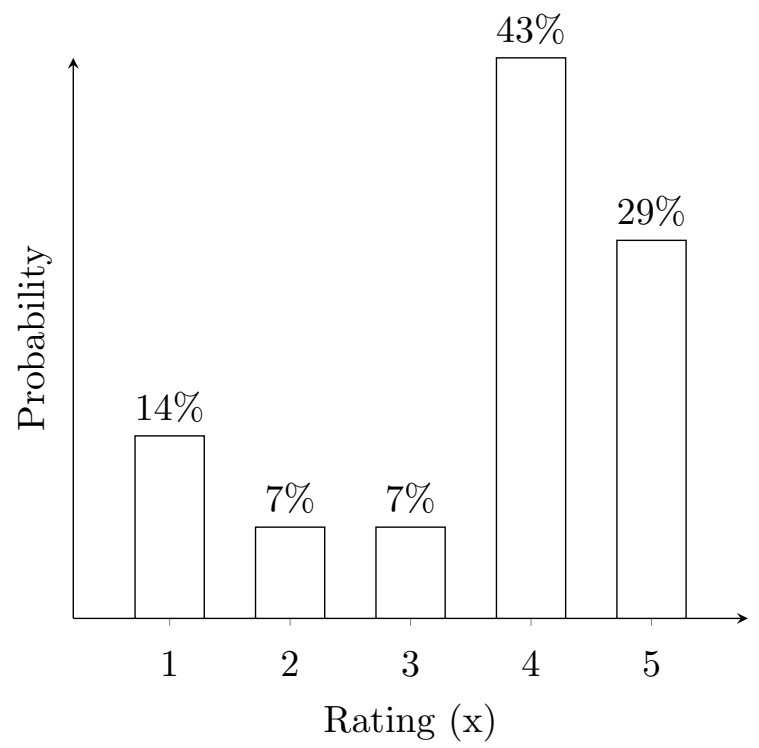

Figure 5.10: Histogram for IDS capability to monitor concrete consistency

\subsection{Laser scanner for applications other than dimen- sioning}

The aforementioned construction activities (monitored with laser scanner) rely on dimensioning accuracy during monitoring. However, in this Section a laser scanner is considered for quality checks that are not primarily reliable on dimensioning accuracy. These checks are as follows:

- Identification of formwork and shuttering condition

- Alignment of formwork.

The diagram in Figure 5.11 shows the content of this Section relative to the other sections in this Chapter. 


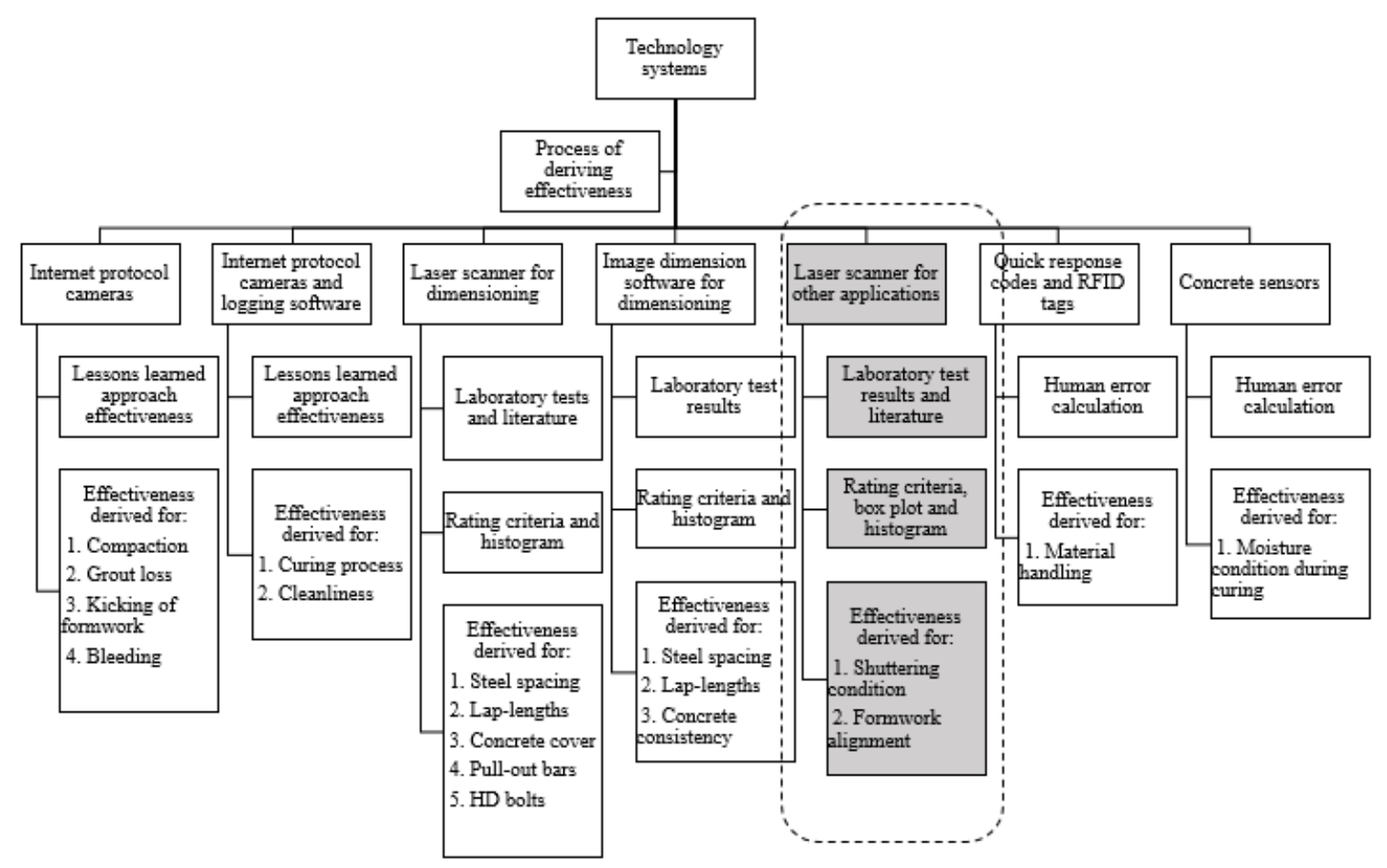

Figure 5.11: Content of this section relative to other Sections in this Chapter

\subsubsection{Identification of formwork and shuttering condition}

Shutter boards get damaged on site and deform with a change in temperature. Ensuring that the shutter surface is without deformations is an important quality check. The deformation of the boards lead to poor finishing and inadequate concrete cover. A laser scanner can identify deformed surfaces on the shuttering through use of heat maps.

Table 5.7 shows the rating criteria for the quality check under consideration. However, the rating criteria is only presented as an example of what could be expected from technology. It was not used to derive the effectiveness of the laser scanner to prevent errors from surface deformations. 
Table 5.7: Rating criteria defined for the monitoring of shutter surface deformations

\begin{tabular}{cl}
\hline Rating criteria & Description \\
\hline 1 & Poor visual available \\
\hline 2 & $\begin{array}{l}\text { Average visual available; } \\
\text { processing time less than } 2 \\
\text { hours }\end{array}$ \\
\hline \multirow{2}{*}{3} & $\begin{array}{l}\text { Clear visual available; } \\
\text { processing time less than 1 } \\
\text { hour }\end{array}$ \\
\hline & $\begin{array}{l}\text { Clear visual available; } \\
\text { processing time less than } 45 \\
\text { minutes }\end{array}$ \\
\hline & $\begin{array}{l}\text { Excellent visual available; heat } \\
\text { maps clearly show } \\
\text { deformations; processing time } \\
\text { less than 30 minutes }\end{array}$ \\
\hline
\end{tabular}

\subsubsection{Laser scanner heat map function to identify surface deformation}

In a study by Kim et al. (2014), a laser scanner was used to identify surface deformations on concrete elements. Kim et al. (2014) identified the surface deformation of 24 surface samples and then determined the accuracy of the identified locations. Most of the test results were accurate within $1 \%$ when determining the position of the deformations.

Through use of a box plot in "Statistica", the author determined a range of deviation percentages from which outliers are excluded. Four out of the 24 results were identified as outliers. Figure 5.12 shows these values on a box plot.

When calculating the effectiveness of the scanner to identify deformations, the outliers are used as an indication that the scanner failed to determine the deformation locations successfully. Since four outliers were identified, it is assumed that the scanner failed 4 out of 24 times to effectively determine the location of the deformations. This provides an assumed effectiveness of $83 \%(20 / 24)$ when using the laser scanner to identify surface deformations.

The non-outlier range includes deviation percentages between $0.006 \%$ to $2.69 \%$. This represents surface defects that were successfully identified. It is reasoned by the author that the quality check is not reliant on the measurement of dimensions, but on the identification of the correct locations. Therefore, accuracy deviations for surface deformations that are within $3 \%$ are assumed to be acceptable. 


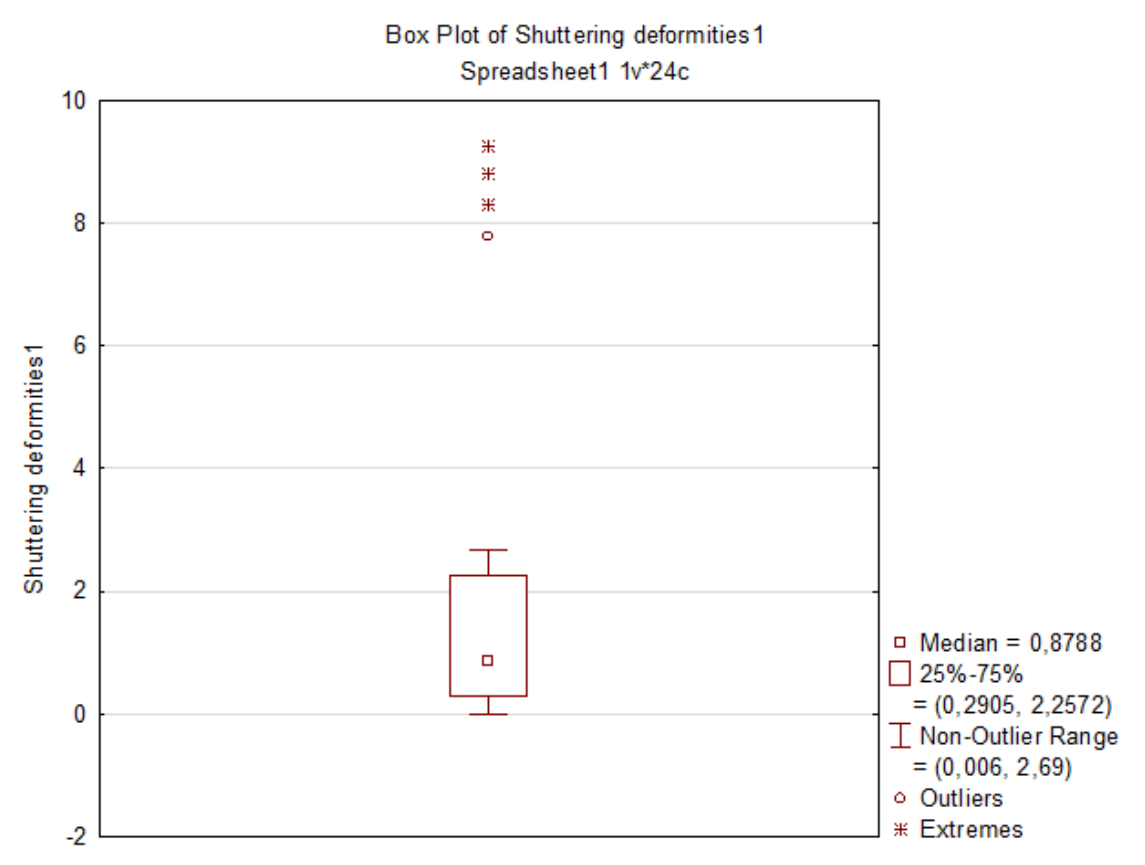

Figure 5.12: Box plot, as determined by the author, to identify outliers and then calculate technology effectiveness

\subsubsection{Dimensions and alignment of formwork}

The dimensions and alignment of formwork is another critical activity in the construction process. Incorrect alignment and dimensions lead to excessive rework since in some cases it is required that elements be reconstructed entirely.

The rating criteria for dimensions and alignment is similar to that established for pullout bars. Both require good accuracy and are so critical that more time is allocated towards the monitoring process. However, support software is required for the alignment check. The support software enables the user to compare the alignment required by the design with the alignment of as-built construction. Table 5.8 shows the rating criteria for technology requirements to monitor the dimensions and alignment of formwork. 
Table 5.8: Rating criteria defined for the monitoring of dimensions and alignment of formwork

\begin{tabular}{|c|c|}
\hline Rating criteria & Description \\
\hline 1 & $\begin{array}{l}\text { Accuracy deviation }>3 \% \\
\text { and/or Processing time }>2 \\
\text { hours }\end{array}$ \\
\hline 2 & $\begin{array}{l}\text { Accuracy deviation }<3 \% \\
\text { and/or Processing time }<1.5 \\
\text { hours }\end{array}$ \\
\hline 3 & $\begin{array}{l}\text { Accuracy deviation }<2 \% \\
\text { and/or Processing time }<1 \\
\text { hour }\end{array}$ \\
\hline 4 & $\begin{array}{l}\text { Accuracy deviation }<1 \% \text { and } \\
\text { Processing time }<45 \text { minutes. } \\
\text { Support software compatible } \\
\text { with technological solution. }\end{array}$ \\
\hline 5 & $\begin{array}{l}\text { Accuracy deviation }<0.5 \% \text { and } \\
\text { Processing time }<30 \text { minutes } \\
\text { and system captures all the } \\
\text { required detail. Support } \\
\text { software compatible with } \\
\text { technological solution. }\end{array}$ \\
\hline
\end{tabular}

\subsubsection{Laser scanner to monitor dimensions and alignment}

A laser scanner can be used to monitor the dimensions and alignment of formwork. To compare the alignment of the as-built construction with the designs, building information modelling (BIM) software is used. Thus, it is recommended that a laser scanner be used for checking the alignment only if a BIM model is available of the design. The histogram produced for the other quality checks in Chapter 5.4 was used for the dimensions and alignment check since they have similar rating criteria. The histogram shows that the laser scanner has a $57 \%$ estimated probability of successfully monitoring the dimensions and alignment with a rating $\geq$ to 4 out of 5 .

\subsection{Quick response code and radio frequency identi- fication effectiveness}

Quick response(QR) codes and radio frequency identification (RFID) tags are proposed to assist in the material handling process. The diagram in Figure 5.13 shows the content of this Section relative to the other Sections in this Chapter. 


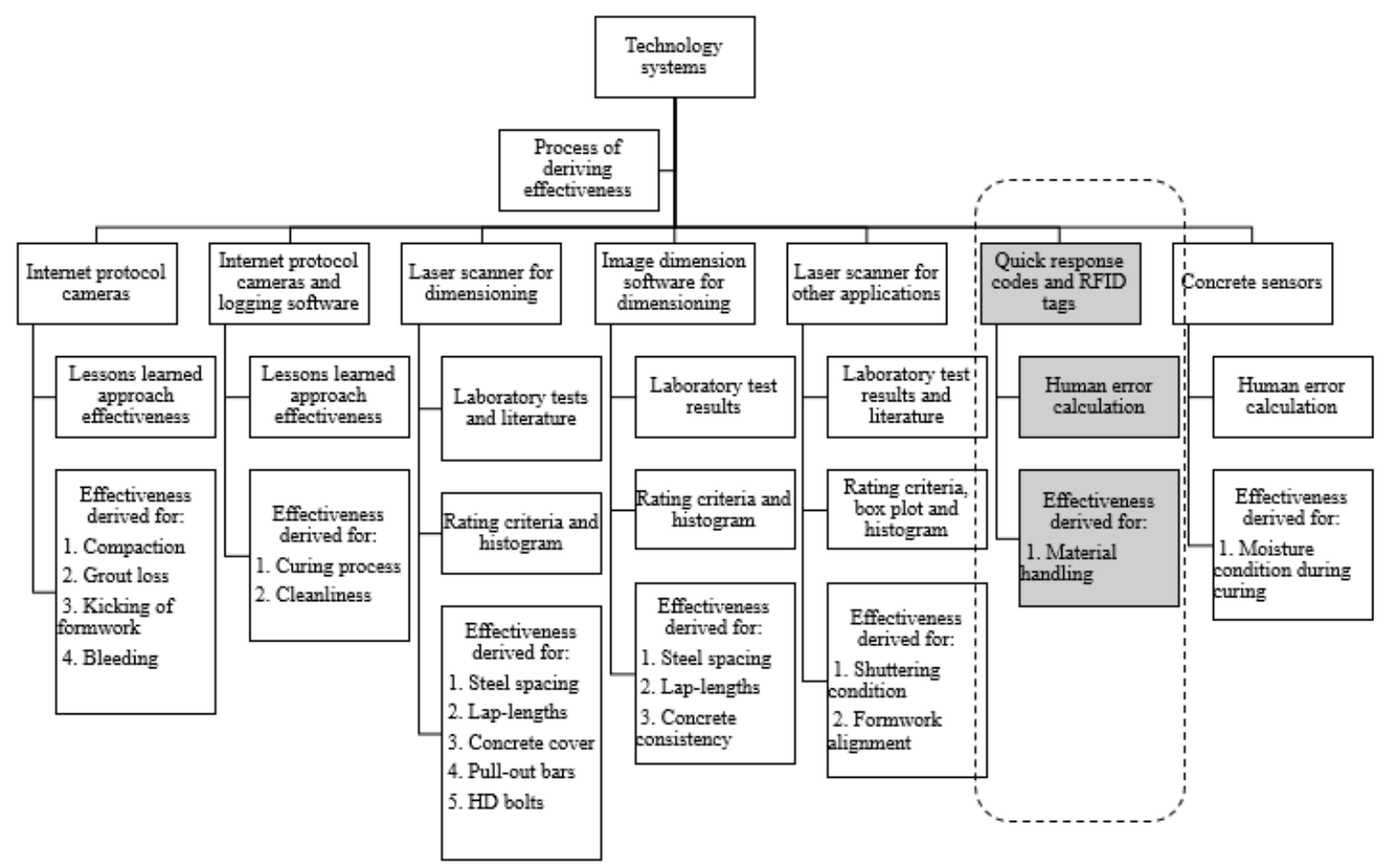

Figure 5.13: Content of this Section relative to content of other Sections in this Chapter

\subsubsection{Material handling}

The use of QR codes and RFID tags in material handling was proposed as an incentive to increase the quality control over material handling on a construction site. The technology assists in reducing the confusion about the location of reinforcement on site. By adopting such a system it is assumed that all the reinforcement would be distributed successfully to its required locations and that system failure is unlikely. However, the system relies on human input to be executed successfully. If the technology is used incorrectly because of misinterpretation or a lack of understanding, the technology usage would be unsuccessful. Therefore, it is reasoned that the incorrect usage of this technology could be as a result of human error.

A factor of human error is calculated according to the Human Error Assessment and Reduction Technique (HEART) that was developed by Williams (1988). This technique is predominantly used in the United Kingdom Nuclear Power and Reprocessing industries. A study by Kirwan, Kennedy and Taylor-adams (1997) compared real human error probabilities with results from the HEART test to validate the technique. The results showed significant correlation, supporting the predicative accuracy of the technique. The result lends support to the empirical validity of the approach.

It is assumed that the other technological systems that were considered, already include human error since the effectiveness was derived from pre-determined data and case studies.

The HEART methodology consists of five steps to determine a probability of failure for a specific task: 
- Generic task unreliability probability

- Identify factors that increase unreliability

- Proportion of effect

- Assessed impact value

- Overall probability of failure due to human error.

This process was followed and is shown in the following steps below.

\subsubsection{Step 1: Generic task unreliability}

In the first step of the HEART technique, the generic task was identified from a list provided in the HEART test. The task was identified as a "Fairly simple task performed rapidly or given scant attention." The proposed nominal human unreliability for this task is equal to 0.09 .

\subsubsection{Step 2: Factors that increase unreliability}

Factors are provided by Williams (1988), that increase unreliability of a specific task. From the list of factors, a few was identified that could relate to this specific construction activity of material handling. These factors, together with the score of each, are shown in Table 5.9. Furthermore, Table 5.9 shows the proportion of effect, which is only applicable in step 3.

Table 5.9: Factors that increase unreliability and the proportion of the effect of each

\begin{tabular}{lcc}
\hline Error-producing condition & $\begin{array}{c}\text { Change in nominal } \\
\text { unreliability }\end{array}$ & Step 3: Proportion of effect \\
\hline 1. Operator inexperienced & 3 & 0.6 \\
\hline $\begin{array}{l}\text { 2. A mismatch between } \\
\text { educational achievement level } \\
\text { of an individual and the } \\
\text { requirements of the task }\end{array}$ & 2 & 0.3 \\
\hline 3. Low workforce morale & & 0.1 \\
\hline
\end{tabular}

\subsubsection{Step 3: Estimated assessed proportion of effect}

In step three it is required to provide a proportion of effect for each of the error producing conditions. The proportion of effect is used to assess the impact of each error-producing condition. The proportion of effect should be provided based on own judgement. The chosen proportions of effect are shown in Table 5.9 .

The author reasons that for the task of material handling, the inexperience of the operator should have the greatest influence on the task. The educational level also has an impact, but since the task under consideration is fairly simple, it does not have such a significant effect. The workforce morale obtained the lowest proportion of effect since morale does not significantly influence the skill level required. 


\subsubsection{Step 4: Calculate assessed effect}

In step four the assessed impact for each of the error-producing conditions are determined by means of equation $(5.2)$.

$$
\text { Effect }=((\text { Multiplier }-1) \times \text { Proportion of effect })+1
$$

The assessed effect is calculated for each of the error producing conditions. Table 5.10 shows the calculated assessed effect for each of the conditions.

Table 5.10: Factors that increase unreliability and the proportion of the effect of each

\begin{tabular}{lccc}
\hline $\begin{array}{l}\text { Error-producing } \\
\text { condition }\end{array}$ & $\begin{array}{c}\text { Change in nominal } \\
\text { unreliability } \\
\text { (Multiplier) }\end{array}$ & $\begin{array}{c}\text { Step 3: Proportion } \\
\text { of effect }\end{array}$ & $\begin{array}{c}\text { Step 4: Assessed } \\
\text { effect }\end{array}$ \\
\hline $\begin{array}{l}\text { 1. Operator } \\
\text { inexperienced }\end{array}$ & 3 & 0.6 & 2.2 \\
\hline $\begin{array}{l}\text { 2. A mismatch } \\
\text { between educational } \\
\text { achievement level of an } \\
\text { individual and the } \\
\begin{array}{l}\text { requirements of the } \\
\text { task }\end{array}\end{array}$ & 2 & 0.3 & 1.3 \\
\hline $\begin{array}{l}\text { 3. Low workforce } \\
\text { morale }\end{array}$ & 1.6 & & \\
\hline
\end{tabular}

\subsubsection{Step 5: Human error probability}

The overall human error probability for the specific task is then calculated by means of Equation (5.3).

$$
\text { Nominal unreliability } \times \text { Assessed impact } 1 \times \ldots \times \text { Assessed impact } n
$$

The $\mathrm{n}$ in equation 5.3 refers to the number of assessed impacts. From Step 1 it was determined that the nominal human unreliability is 0.09 and the assessed impacts are shown in Table 5.10 ,

The calculated human error probability is 0.2728 .

It is assumed that this represents a $27.28 \%$ chance of human error leading to the unsuccessful distribution of reinforcement when using technology such as RFID tags and QR codes. The probability that the task is successfully executed is $72.72 \%$. This will be used as the probability that the technological system would successfully be used to mitigate the risk of using the incorrect reinforcement.

\subsection{Concrete curing sensor effectiveness}

The use of concrete curing sensors was proposed as a method to monitor the moisture condition of concrete during curing. The diagram in Figure 5.14 shows the content of this Section relative to the other Sections in this Chapter. 


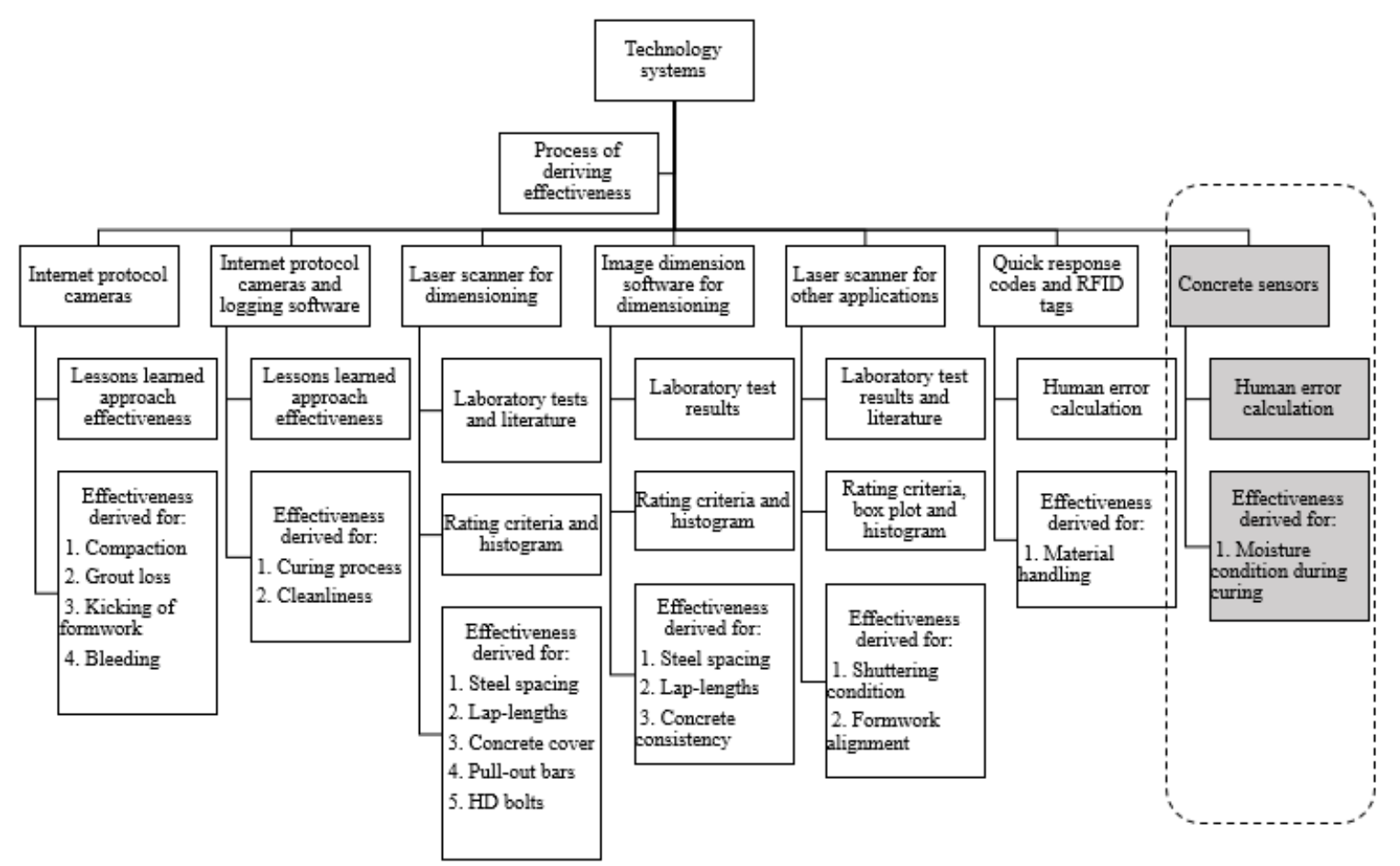

Figure 5.14: Content of this Section relative to content of other Sections in this Chapter

\subsubsection{Moisture condition during curing}

It is beneficial to monitor the relative humidity and expected strength of the concrete during curing. Profit potential is created by providing the user with curing data that will improve decision-making and therefore saves time and money (Sangregario, 2012). The only technological system identified for this purpose is the concrete curing sensors. Little information was found where the sensors have been used and evaluated. The sensors are used during curing to provide an indication of the concrete curing condition. It is reasoned that if information on the curing process assists the contractor to improve quality control then rework caused by poor curing is reduced.

A study by Sangregario (2012) showed that the sensors successfully tracked temperatures and moisture content while embedded. For this study it was assumed that the technical specifications of the technology is consistent and accurate.

To determine the effectiveness of the system to improve quality control, human error is considered. Human error could occur during the installation and operation of the system. The human error calculation, that was previously completed for material handling, was used for the concrete sensors. The human error probability previously calculated was $27.28 \%$. Therefore, the estimated probability of the system preventing rework that results from curing errors is is $72.72 \%$. 


\subsection{Summary of technology effectiveness}

The author investigated the effectiveness of technology systems for rework reduction in construction. However, the effectiveness values were determined through approaches which potentially do not represent the true effectiveness of the technologies. This can only be determined after testing the technology on a number of real-life projects. The author could not test the technologies in such a manner because of time and financial constraints. Furthermore, new technologies and improvements to current technologies occur over short time periods. Thus, for this study the emphasis is placed on the logic of the decision model that provides investment suggestions of technology to users (Chapter 7).

The effectiveness values can be determined through other methods if desired. An example of this is shown in Appendix C, where the effectiveness of the laser scanner and IDS was derived from probability density functions(PDFs). The PDFs were developed through use of Kolmogorov-Smirnov and Anderson-Darling goodness-of-fit tests. Companies presenting its technological solutions could use its own testing methods and effectiveness criteria to determine the capability of their technology. The effectiveness values would then be used in the decision model.

Table 5.11 shows a summary of the effectiveness determined for each of the technologies to prevent the identified construction errors. The effectiveness of the technological systems are used in the investment decision model (presented in Chapter 7). It is used to reduce the potential savings that could realise when using these technologies to mitigate construction risks.

Table 5.11: Summary of the effectiveness determined for each of the technological solutions to monitor the identified construction errors that could occur

\begin{tabular}{llc}
\hline Construction error & Technological system & $\begin{array}{c}\text { Estimated probability } \\
\text { of success (\%) }\end{array}$ \\
\hline Steel spacing & $\begin{array}{l}\text { Laser scanner } \\
\text { Image dimensioning } \\
\text { software (IDS) }\end{array}$ & 57 \\
\hline Compaction & IP camera & 72 \\
\hline Grout loss & IP camera & 55 \\
\hline Concrete cover & Laser scanner & 55 \\
\hline Kicking of formwork & IP camera & 57 \\
\hline Bleeding & IP camera & 55 \\
\hline Consistency of mix & IDS & 55 \\
\hline Material handling & QR code/RFID & 72 \\
\hline Moisture condition during & Concrete sensors & 73 \\
curing & IP camera & 73 \\
\hline Curing process & Laser scanner & 55 \\
\hline Lap-lengths & IDS & 57 \\
\hline Pull-out bars & Laser scanner & 72 \\
\hline
\end{tabular}




\begin{tabular}{llc}
\hline $\begin{array}{l}\text { Identification of formwork } \\
\text { and shuttering condition }\end{array}$ & Laser scanner & 83 \\
\hline $\begin{array}{l}\text { Cleanliness of equipment } \\
\text { and formwork }\end{array}$ & IP camera & 55 \\
\hline $\begin{array}{l}\text { Dimensions and alignment } \\
\text { of formwork }\end{array}$ & Laser scanner & 57 \\
\hline Position of HD bolts & Laser scanner & 57 \\
\hline
\end{tabular}




\section{Chapter 6}

\section{Questionnaire Survey}

Figure 6.1 shows the scope of this Chapter relative to the other Chapters.

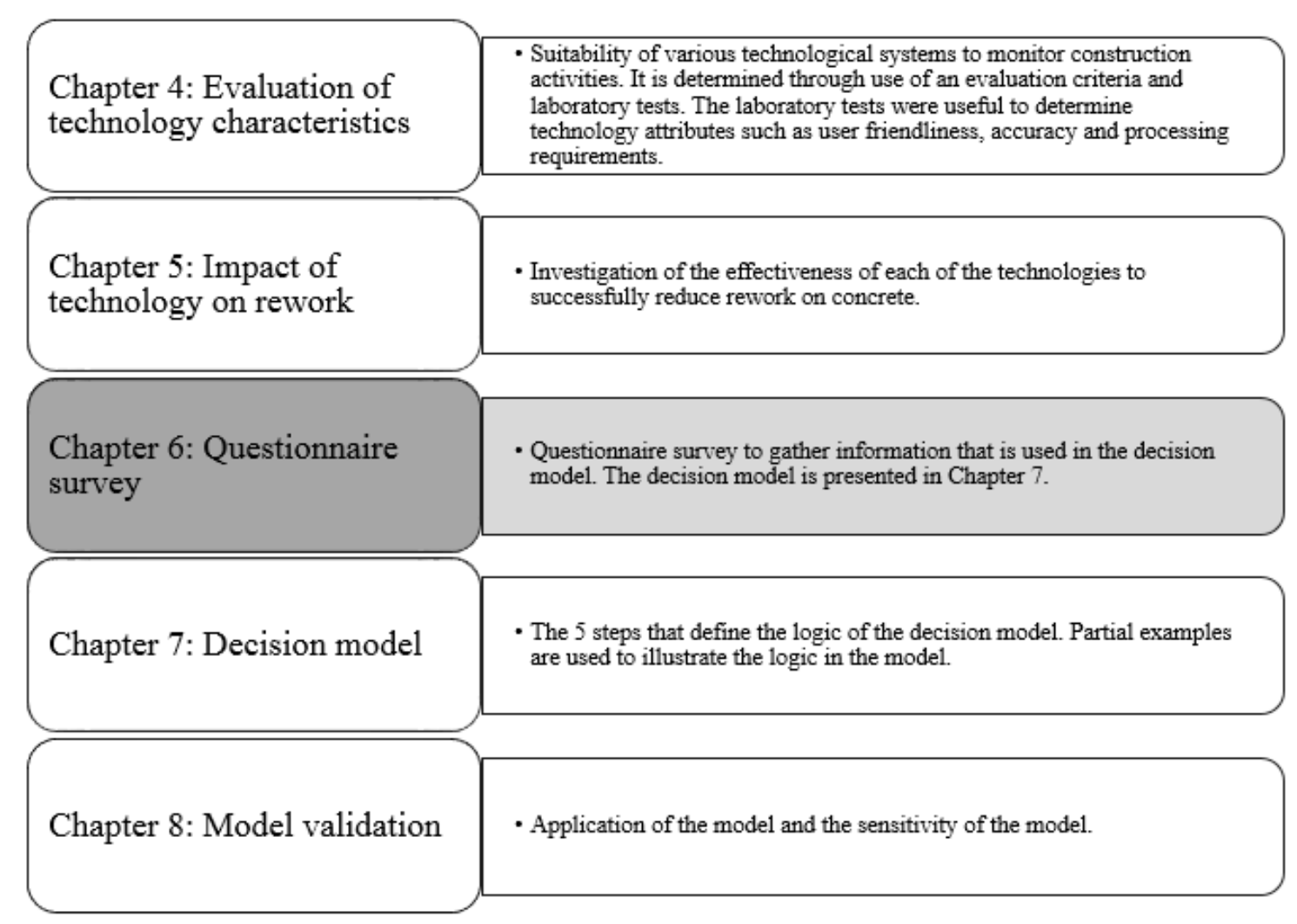

Figure 6.1: Scope of Chapter 6

This Chapter presents the data that was obtained through a questionnaire survey which was designed on "Survey monkey." The aim of the survey is to obtain information that is used in the development of the decision model in Chapter 7. The information is used in the model to quantify the risk magnitude of construction errors that lead to rework. Participants were approached through a personal e-mail which included the link to the questionnaire survey. 


\subsection{Participant information}

The questionnaire was registered for Continuing Professional Development (CPD) points, with the Engineering Council of South Africa (ECSA), by presenting it as a short online lecture. The reason for CPD registration was to motivate industry experts to complete the survey so that more responses can be collected. The participants were provided with a brief background to the study to gain a better understanding of the questions. The short lecture and questions are shown in Appendix II. A total of 41 industry participants responded to the questionnaire. Table 6.1 shows a summary of the participant information.

Participants were identified from a list of industry experts that previously attended courses at Stellenbosch University. Participants that are currently in the engineering contracting and -consulting industry were approached by means of e-mail. The e-mail invited the individuals to participate in the survey and provided them with a brief explanation of how they can earn CPD points by completing the survey. In the e-mail, participants had to follow a link to an online questionnaire survey. The survey complied with the requirements of the Stellenbosch University Ethics Approval Committee (SU-HSD-003014); therefore, participants had the right to withdraw from the survey at any time they wished to.

Table 6.1: Participant information

\begin{tabular}{lccc}
\hline Discipline & Number of participants & $\begin{array}{c}\text { Average Contracting } \\
\text { experience (years) }\end{array}$ & $\begin{array}{c}\text { Average Consulting } \\
\text { experience (years) }\end{array}$ \\
\hline Only Contracting & 14 & 18.57 & 0.00 \\
\hline Only Consulting & 8 & 0.00 & 18.63 \\
\hline $\begin{array}{l}\text { Contracting and } \\
\text { Consulting }\end{array}$ & 17 & 15.85 & 9.65 \\
\hline Total Contracting & 31 & 17.00 & - \\
\hline Total Consulting & 25 & - & 12.50 \\
\hline
\end{tabular}

In total, 31 of the respondents have contracting experience. Out of the 31 participants with contracting experience, 14 have zero consulting experience. The other 17 participants have both contracting and consulting experience.

In total, 25 respondents have consulting experience. Out of the 25 participants with consulting experience, 17 have consulting and contracting experience. The other 8 participants have zero contracting experience.

Of the 31 respondents with contracting experience, the average contracting experience in years was 17 years. Of the 25 respondents with consulting experience, the average consulting experience was 12.5 years.

Two of the participants did not enter their relevant experience. Therefore, participant experience information was only available for $39(14+8+17)$ of the 41 participants. 


\subsection{Questionnaire results}

Tables 6.2 to 6.5 show the results obtained through Questions 1-4. Questions 1-4 obtained information regarding the influence of attributes of "Labour, Management, Subcontractors, and Plant and Equipment" on the quality of structural concrete. On a project, these attributes determine the performance expected from each of the categories. The results obtained through Questions 1-4 were used to determine a weight for each of the attributes in its relevant category. The weights ensure that the influence of each attribute is proportionately applied in the decision model.

An independent two sample t-test was used to determine the variability between the data obtained from contractors and consultants. The t-test is used to indicate whether there is a significant difference between the means of two samples. Sample 1 includes the results from consultants and sample 2 includes the results from contractors. For all the t-tests the null- and alternative hypotheses are as follows:

$H_{0}: \mu_{1}=\mu_{2}$ and $H_{a}: \mu_{1} \neq \mu_{2}$

The null hypothesis $\left(H_{0}\right)$ states that the mean of population 1 and 2 are equal $\left(\mu_{1}=\mu_{2}\right)$. The alternative hypothesis $\left(H_{a}\right)$ states that the mean of population 1 and 2 are not equal $\left(\mu_{1} \neq \mu_{2}\right)$.

The steps to consider in the t-test are shown in Chapter 3. A significance value $(\alpha)$ of 0.05 was used (this provides a $95 \%$ confidence interval). The p-value obtained from the t-test (through "Statistica") was used as the test statistic. Thus, if the p-value is $\geq 0.05$ the null hypothesis is not rejected. In other words, the test statistic lends support to the assumption that there is no significant differences in the mean of the answers provided by contractors and engineers.

Most of the t-tests conducted for the survey data showed there was not a significant difference $(\mathrm{p} \geq 0.05)$ between the data provided by contractors and consultants. Since most of the p-values exceeded a value of $\geq 0.05$ it is thus concluded that the data obtained through the questionnaire survey was reliable. Ultimately, the data obtained from contractors and consultants did not show a significant difference.

Each question is followed by their results, which are ranked according to its mean rating from highest to lowest. The result Tables also show the standard deviation of the results, weights determined from the results and the p-values obtained from the t-test. The weights of the results are used in the decision model which is presented in Chapter 7. The questions of the survey are shown below.

"1. Provide a rating between zero and ten (0-10) for the influence of each Labour attribute on the quality of structural concrete during construction. Zero means no influence and ten means it has an important influence on the quality of structural concrete." 
Table 6.2: Results from Question 1

\begin{tabular}{lcccc}
\hline Attributes & $\begin{array}{c}\text { Rating mean } \\
(/ \mathbf{1 0})\end{array}$ & Std Dev & Weight(w) & t-test p-value \\
\hline Skill level & 8.34 & 1.543 & 0.211 & 0.35 \\
\hline Training & 8.33 & 1.439 & 0.210 & 0.25 \\
\hline $\begin{array}{l}\text { High ratio of } \\
\text { casual labour } \\
\text { versus permanent } \\
\text { labour }\end{array}$ & 7.71 & 2.015 & 0.195 & 0.37 \\
\hline $\begin{array}{l}\text { Motivation of } \\
\text { labourers and } \\
\text { supervisor }\end{array}$ & 7.63 & 1.854 & 0.193 & 0.85 \\
\hline Self-discipline & 7.61 & 2.143 & 0.192 & 0.25 \\
\hline
\end{tabular}

From Table 6.2 it is seen that the skill level of the labour ranked the highest and the self-discipline ranked the lowest. However, there was not significant differences in the mean values of each.

"2. Provide a rating between zero and ten (0-10) for the influence of each Management attribute on the quality of structural concrete during construction. Zero means no influence and ten means it has an important influence on the quality of structural concrete."

Table 6.3: Results from Question 2

\begin{tabular}{lcccc}
\hline Attributes & $\begin{array}{c}\text { Rating mean } \\
(/ \mathbf{1 0})\end{array}$ & Std Dev & Weight(w) & t-test p-value \\
\hline Poor supervision & 8.98 & 1.332 & 0.159 & 0.70 \\
\hline $\begin{array}{l}\text { Poor } \\
\text { communication }\end{array}$ & 8.44 & 1.566 & 0.150 & 0.99 \\
\hline Coordination & 8.05 & 1.548 & 0.143 & 0.44 \\
\hline $\begin{array}{l}\text { Lack of a Quality } \\
\text { Management }\end{array}$ & 7.95 & 2.269 & 0.141 & 0.51 \\
$\begin{array}{l}\text { System } \\
\begin{array}{l}\text { Impractical } \\
\text { design }\end{array}\end{array}$ & 7.76 & 2.047 & 0.137 & 0.49 \\
\hline $\begin{array}{l}\text { Precision } \\
\text { planning }\end{array}$ & 7.63 & 1.894 & 0.135 & 0.85 \\
\hline $\begin{array}{l}\text { Lack of quality } \\
\text { management } \\
\text { expertise }\end{array}$ & 7.61 & 2.072 & 0.135 & \\
\hline
\end{tabular}

From Table 6.3 it is seen that poor supervision ranked the highest and that a lack of quality management expertise ranked the lowest. However, there was not significant differences in the mean ratings obtained through Question 2.

"3. Provide a rating between zero and ten (0-10) for the influence of Subcontractor attributes on the quality of structural concrete during construction. Zero means no influence and ten means it has an important influence on the quality of structural concrete." 
Table 6.4: Results from Question 3

\begin{tabular}{lcccc}
\hline Attributes & $\begin{array}{c}\text { Rating mean } \\
(/ \mathbf{1 0})\end{array}$ & Std Dev & Weight(w) & t-test p-value \\
\hline Experience & 8.45 & 1.554 & 0.267 & 0.36 \\
\hline $\begin{array}{l}\text { Knowledge of } \\
\text { operations }\end{array}$ & 8.03 & 1.568 & 0.253 & 0.36 \\
\hline $\begin{array}{l}\text { Construction } \\
\text { method }\end{array}$ & 7.76 & 2.059 & 0.245 & 0.21 \\
\hline $\begin{array}{l}\text { Communication } \\
\text { skills }\end{array}$ & 7.45 & 1.639 & 0.235 & 0.056 \\
\hline
\end{tabular}

From Table 6.4 it is seen that the experience of the subcontractor ranked the highest and the the communication skills ranked the lowest. However, there was not a significant difference in the mean values obtained through Question 3.

"4. Please provide a rating between zero and ten (0-10) for the influence of Plant and Equipment attributes on the quality of structural concrete during construction. Zero means no influence and ten means it has an important influence on the quality of structural concrete."

Table 6.5: Results from Question 4

\begin{tabular}{lcccc}
\hline Attributes & $\begin{array}{c}\text { Rating mean } \\
(/ \mathbf{1 0})\end{array}$ & Std Dev & Weight(w) & t-test p-value \\
\hline $\begin{array}{l}\text { Cleanliness of } \\
\text { formwork and } \\
\text { shutter boards }\end{array}$ & 8.44 & 1.747 & 0.348 & 0.85 \\
\hline $\begin{array}{l}\text { Equipment that } \\
\text { is sub-standard }\end{array}$ & 8.02 & 1.710 & 0.331 & 0.03 \\
\hline $\begin{array}{l}\text { Machinery used is } \\
\text { old and poorly } \\
\text { maintained }\end{array}$ & 7.8 & 1.792 & 0.322 & 0.08 \\
\hline
\end{tabular}

From Table 6.5 it is seen that the cleanliness of formwork and shutter boards ranked the highest and machinery that is old and poorly maintained ranked the lowest. However, there is not a significant difference in the magnitude of the means that were obtained through Question 4. Note that the p-value for sub-standard equipment is below the 0.05 significance value. Theoretically it cannot be assumed that the information provided by contractors and consultants are similar to provide reliable information. However, for this study it is assumed that the information is reliable.

Questions 5-8 were used to find the influence of "Labour, Management, Subcontractors and Plant and Equipment" on the identified construction activities for structural concrete works that mainly consist of large footings, such as bases for wind generators. The results were not used in this study since the scope was narrowed down to include only structural elements such as beams, columns, slabs and reinforced walls. In Question 13 it is shown the rework expectancy on projects that consist of large bases is much lower compared to projects that mainly consist of structural elements such as beams, columns, slabs and reinforced walls. 
Questions 9-12 were used to find the influence of "Labour, Management, Subcontractors and Plant and Equipment" on the identified construction activities for structural concrete works that mainly consist of structural elements, such as beams, columns and slabs.

The participants answered Questions 9-12 according to a fixed scale ranging from "Negligible" to "Severe." In order to use the results in the risk magnitude calculation, presented in Chapter 7, the qualitative categories were converted to numerical values. For example, if most of the participants answered "Severe(5)" for one of the questions then a score would be obtained that ranges between 4 and 5 . The reason therefore is that some of the participants would have chosen "Significant(4)" or "Moderate(3)" which produces a score lower than 5. Table 6.6 shows the qualitative descriptions with their scores as they are converted from the survey. Table 6.6 also shows the classification of the answers after calculating the mean value from all the participants. However, the classification does not have an influence on the risk magnitude calculations (Chapter 7 ) since only the numerical values are used.

Table 6.6: Risk impact criteria in numerical values

\begin{tabular}{lcc}
\hline $\begin{array}{l}\text { Impact } \\
\text { description }\end{array}$ & $\begin{array}{c}\text { Numerical } \\
\text { conversion }\end{array}$ & Mean range \\
\hline Negligible & 1 & $\mathrm{x}=1$ \\
\hline Minor & 2 & $1<\mathrm{x} \leq 2$ \\
\hline Moderate & 3 & $2<\mathrm{x} \leq 3$ \\
\hline Significant & 4 & $3<\mathrm{x} \leq 4$ \\
\hline Severe & 5 & $4<\mathrm{x} \leq 5$ \\
\hline
\end{tabular}

Tables 6.7 to 6.10 show the mean score obtained for the influence of labour, management, subcontractors, and plant and equipment on the various construction errors identified. The mean scores are ranked from highest to lowest and their matching qualitative description (found in Table 6.6) are shown. The p-value was determined for each of the questions through use of the t-test. Note that weights were not determined for these results since it is not required in the decision model.

Question 9-12 are shown below.

"9. Select (from own expert opinion) the influence of Labour on the identified quality aspects for the construction of other structural elements (for example: beams, columns, slabs)" 
Table 6.7: Results from Question 9

\begin{tabular}{lccc}
\hline $\begin{array}{l}\text { Construction } \\
\text { errors }\end{array}$ & Rating mean (/5) & Impact description & t-test p-value \\
\hline Compaction incorrect & 3.98 & Significant & 0.005 \\
\hline $\begin{array}{l}\text { Incorrect cover } \\
\text { obtained }\end{array}$ & 3.98 & Significant & 0.25 \\
\hline $\begin{array}{l}\text { Kicking of formwork } \\
\text { takes place }\end{array}$ & 3.90 & Significant & 0.38 \\
\hline Steel spacing incorrect & 3.80 & Significant & 0.007 \\
\hline $\begin{array}{l}\text { Grout loss during } \\
\text { pouring }\end{array}$ & 3.73 & Significant & 0.43 \\
\hline
\end{tabular}

From Table 6.7 it is seen that, of the identified construction errors, labour has the greatest impact on incorrect compaction and lowest impact on grout loss during pouring. However, the impact of labour on the identified construction errors are all classified as "Significant." Note that two of the p-values in Table 6.7 are below the significance value of 0.05 . However, it is assumed that the information is still reliable for this study since most of the p-values are above 0.05 .

"10. Select (from own expert opinion) the influence of Management on the identified quality aspects for the construction of other structural elements (for example: beams, columns, slabs)"

Table 6.8: Results from Question 10

\begin{tabular}{lclc}
\hline $\begin{array}{l}\text { Construction } \\
\text { errors }\end{array}$ & Rating mean $(/ \mathbf{5})$ & Impact description & t-test p-value \\
\hline Position of HD bolts & 4.32 & Severe & 0.64 \\
\hline $\begin{array}{l}\text { Incorrect curing } \\
\text { procedures }\end{array}$ & 4.07 & Severe & 0.34 \\
\hline Incorrect lap-lengths & 3.95 & Significant & 0.73 \\
\hline $\begin{array}{l}\text { Pull-out bars } \\
\text { incorrect }\end{array}$ & 3.85 & Significant & 0.17 \\
\hline $\begin{array}{l}\text { Incorrect slump and } \\
\text { consistency }\end{array}$ & 3.80 & Significant & 0.14 \\
\hline $\begin{array}{l}\text { Incorrect distribution } \\
\text { of reinforcement }\end{array}$ & 3.70 & Significant & 0.12 \\
\hline $\begin{array}{l}\text { Grout loss during } \\
\text { pouring }\end{array}$ & 3.49 & Significant & 0.64 \\
\hline Bleeding present & 3.37 & Significant & 0.28 \\
\hline
\end{tabular}

From Table 6.8 it is seen that management has the greatest impact on the position of HD bolts and lowest impact on bleeding of concrete. The impact of management on most of the construction errors under consideration were classified as "Significant."

"11. Select (from own expert opinion) the influence of Subcontractors on the identified quality aspects for the construction of other structural elements (for example: beams, columns, slabs)" 
Table 6.9: Results from Question 11

\begin{tabular}{lccc}
\hline $\begin{array}{l}\text { Construction } \\
\text { errors }\end{array}$ & Rating mean (/5) & Impact description & t-test p-value \\
\hline Incorrect steel spacing & 3.88 & Significant & 0.43 \\
\hline $\begin{array}{l}\text { Incorrect concrete } \\
\text { cover }\end{array}$ & 3.80 & Significant & 0.92 \\
\hline $\begin{array}{l}\text { Incorrect slump and } \\
\text { consistency }\end{array}$ & 3.55 & Significant & 0.13 \\
\hline
\end{tabular}

From Table 6.9 it is seen that, of the construction errors identified that are related to subcontractors, the greatest impact they have are on steel spacing being incorrect. The lowest impact identified is incorrect slump and consistency of the concrete mix. The impact of subcontractors on each of the identified construction errors were classified as "Significant."

"12. Select (from own expert opinion) the influence of Plant and equipment on the identified quality aspects for the construction of other structural elements (for example: beams, columns, slabs)"

Table 6.10: Results from Question 12

\begin{tabular}{lclc}
\hline $\begin{array}{l}\text { Construction } \\
\text { errors }\end{array}$ & Rating mean (/5) & Impact description & t-test p-value \\
\hline $\begin{array}{l}\text { Delays due to } \\
\text { breakages }\end{array}$ & 3.98 & Significant & 0.06 \\
\hline Poor finishing & 3.41 & Significant & 0.62 \\
\hline $\begin{array}{l}\text { Incorrect dimensions } \\
\text { and alignment }\end{array}$ & 3.23 & Significant & 0.66 \\
\hline
\end{tabular}

From Table 6.10 it is seen that plant and equipment has the greatest impact on delays due to breakages and the lowest impact on incorrect dimensions and alignment of the works. However, the mean impact magnitude of plant and equipment shows small differences between each of the construction errors identified.

Question 13 provides an indication of the total expected rework costs on structural concrete works as a percentage of the concrete works on a project. In Table6.11 it is seen that for structural elements the statistical mode is obtained for the $4-6 \%$ range category. The statistical mode represents the category that was chosen the most by the participants. For large footings a statistical mode was obtained for the $0.5-1 \%$ range category. Most of the data in Table 6.11 was determined from the participants' own estimations. From the results in question 13 it is clear that the severity of rework is worse for structural elements; therefore, the model in Chapter 7 is not developed for large footing/bases of structural concrete constructions.

"13. Estimate (from own experience or company information) the expected value of rework on structural concrete as a percentage of the total value of concrete works for the following projects." 
Table 6.11: Expected rework cost on the structural concrete works

\begin{tabular}{|c|c|c|c|c|c|c|c|c|c|c|}
\hline $\begin{array}{l}\text { Answer } \\
\text { options }\end{array}$ & $\begin{array}{l}0.0- \\
0.5 \%\end{array}$ & $\begin{array}{l}0.5- \\
1.0 \%\end{array}$ & $1-2 \%$ & $2-4 \%$ & $4-6 \%$ & $6-8 \%$ & $8-10 \%$ & $>10 \%$ & $\begin{array}{l}\text { Rating } \\
\text { Mode }\end{array}$ & $\begin{array}{l}\text { Mode } \\
\text { Count }\end{array}$ \\
\hline & \multicolumn{8}{|c|}{ Selected by number of participants } & & \\
\hline $\begin{array}{l}\text { Structural } \\
\text { elements }\end{array}$ & 4 & 6 & 4 & 5 & 10 & 7 & 3 & 1 & $4-6 \%$ & 10 \\
\hline $\begin{array}{l}\text { Large } \\
\text { footings } \\
\text { (wind } \\
\text { genera- } \\
\text { tors) }\end{array}$ & 11 & 12 & 4 & 4 & 3 & 1 & 3 & 2 & $0.5-1 \%$ & 12 \\
\hline
\end{tabular}

\subsection{Synthesis}

This Chapter presented the results that were obtained through the questionnaires. The results presented in Tables 6.2 to 6.5 show that the attributes identified under labour, management, subcontractors, and plant and equipment do have a significant impact on quality of concrete as a whole since high ratings were obtained.

From the results presented in Tables 6.7 to 6.10 it was found that labour, management, subcontractors, and plant and equipment have a significant influence on the construction activities that are important to ensure good concrete quality.

The weights obtained through Questions 1-4 are used in Chapter 7to proportionately adjust the user ratings of attributes under labour, management, subcontractors, and plant and equipment. In Chapter 7, the mean values determined from the results of Questions 9-12 are used in the risk magnitude calculation as risk impact values.

An estimation of the rework value on structural concrete work was obtained for works that consist mainly of structural elements and large footings. It was shown that the expected rework on projects that consist mainly of large footings, such as for wind generators, is much less than that of projects that consist mainly of structural elements. Therefore, the scope of the investigation is narrowed down to focus only on concrete works that consist of beams, columns, slabs and structural walls. More rework is potentially reduced on projects where higher rework values are expected. The results of Question 13 showed that the rework expectancy of projects that consist mainly of structural elements, such as beams and columns, are between 4-6\%. According to various authors in literature it was found that these values range between $2.5-20 \%$.

The following Chapter presents the logic of the risk decision model and how the survey results were used to populate the model. 


\section{Chapter 7}

\section{Decision model logic}

Figure 7.1 shows the scope of Chapter 7 relative to the other Chapter in the study.

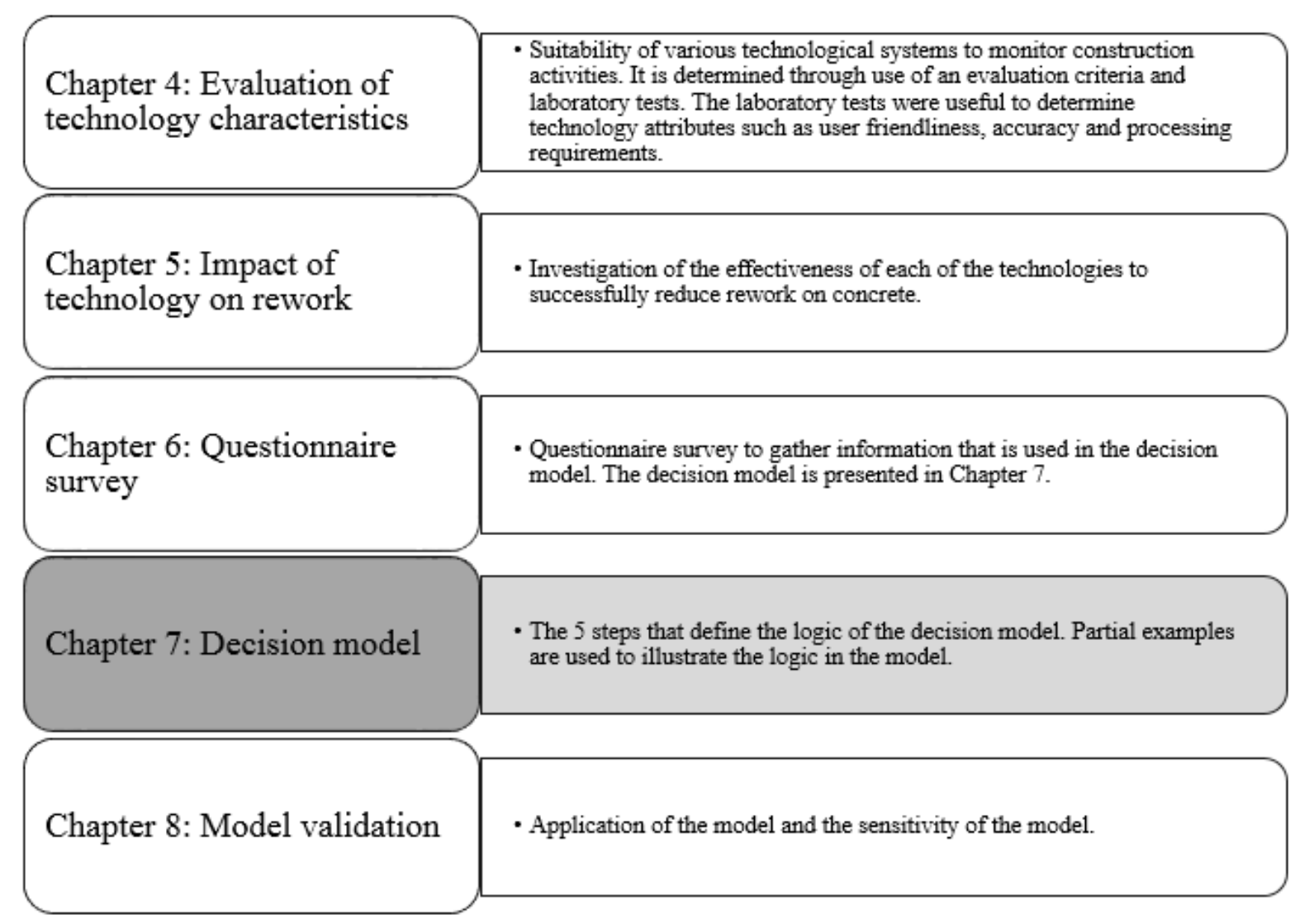

Figure 7.1: Scope of Chapter 7

In this Chapter a decision model is developed based on risk and cost. First, an overview of the model is provided and then the logic of each step of the model is discussed. Various examples are used throughout Chapter 7 to illustrate the use of the model. 


\subsection{Model overview}

The purpose of this model is to assist the contractor with the decision to invest in technological systems that mitigate the risk of construction errors.

The model is based on the principles of risk management. There are processes in the model that use the results from the questionnaire survey (Chapter 6) and processes that require input from the user.

The model comprises of 5 steps. Table 7.1 provides a brief overview of the model. When referring to "category" or "categories" in Table 7.1, it refers to "Labour, Management, Subcontractors or Plant and Equipment." Previously identified attributes, that have an influence on construction quality, are sub-divided between these categories. The steps in the model are described in the order as shown in Table 7.1. Throughout this chapter partial examples are used to illustrate the logic that makes up the model.

Table 7.1: Overview of the risk management model

\begin{tabular}{|c|c|c|c|c|}
\hline Step number & Name of step & Brief summary & $\begin{array}{l}\text { Data from User } \\
\text { input/Model } \\
\text { background }\end{array}$ & Example \\
\hline 1 & Attribute ratings & $\begin{array}{l}\text { User rates various } \\
\text { attributes of } \\
\text { categories to } \\
\text { determine expected } \\
\text { performance on } \\
\text { project. }\end{array}$ & User input & Table 7.4 on $\mathrm{p} 124$ \\
\hline 2 & Category scores & $\begin{array}{l}\text { Weighted average of } \\
\text { each category } \\
\text { determined. }\end{array}$ & Background & Table 7.3 on $\mathrm{p} 127$ \\
\hline 3 & $\begin{array}{l}\text { Determine risk } \\
\text { magnitude }\end{array}$ & $\begin{array}{l}\text { Risk magnitude } \\
\text { determined from } \\
\text { user input and } \\
\text { questionnaire data. }\end{array}$ & Background & $\begin{array}{l}\text { Table } 7.6 \text { on p } 131 \text { to } \\
\text { identify risk } \\
\text { magnitude }\end{array}$ \\
\hline 4 & Risk mitigation & $\begin{array}{l}\text { User chooses which } \\
\text { risks to mitigate. }\end{array}$ & User input & $\begin{array}{l}\text { Examples shown in } \\
\text { Figure } 8.6 \text { on } \mathrm{p} 150\end{array}$ \\
\hline 5 & $\begin{array}{l}\text { Investment } \\
\text { suggestion }\end{array}$ & $\begin{array}{l}\text { Model calculates the } \\
\text { funds to be invested. }\end{array}$ & Background & Section 7.6 on $\mathrm{p} 135$ \\
\hline
\end{tabular}

\subsection{Step 1: Attribute ratings}

Figure 7.2 shows the brief outline of Step 1 relative to the other steps in the model. 


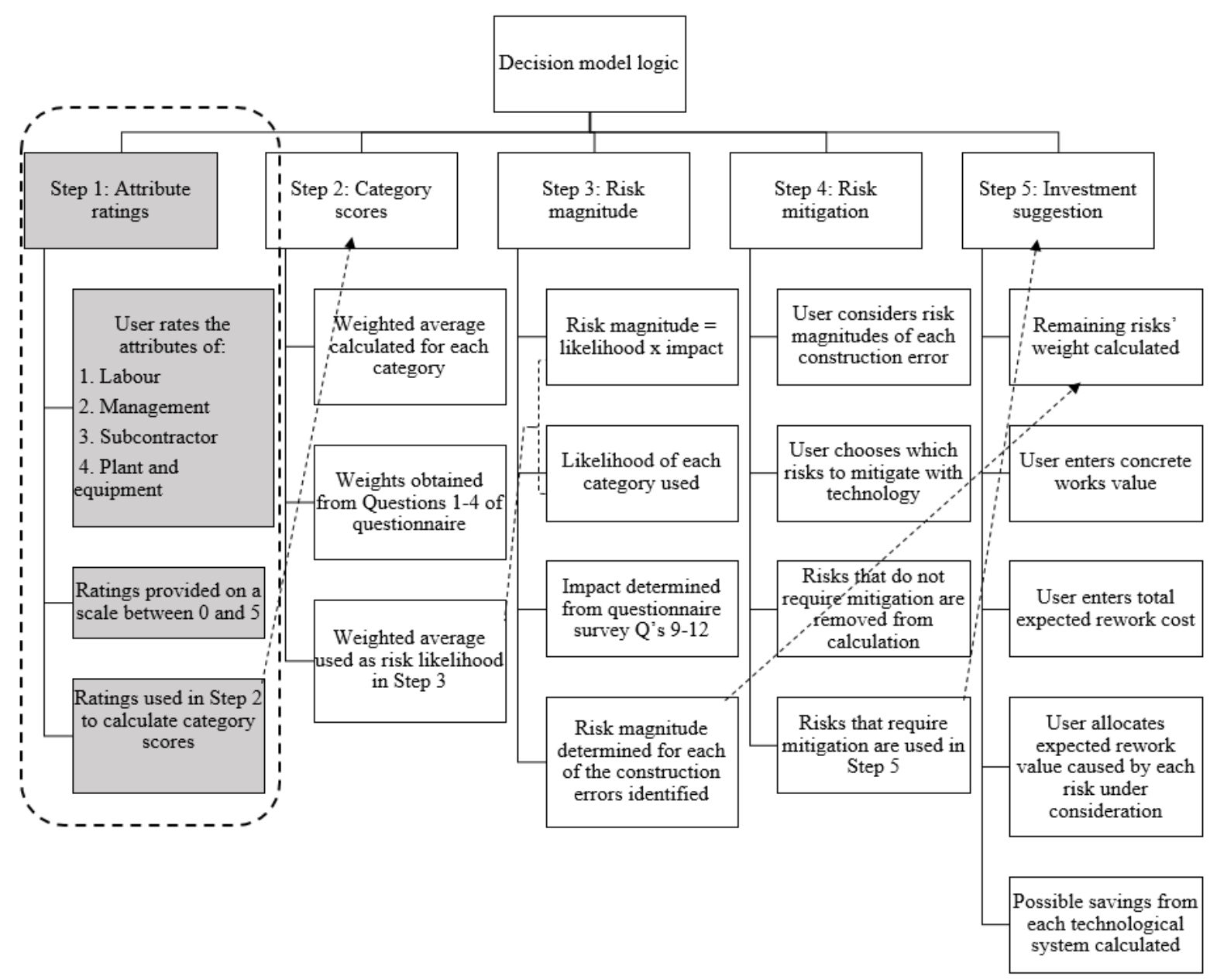

Figure 7.2: Overview of Step 1 in the decision model

The diagram in Figure 7.3 shows the logic of the first step in the model. The ellipse indicates the start or end of a step; the hexagon indicates that user input is required and the rectangle indicates that an action is completed in the background of the model. 


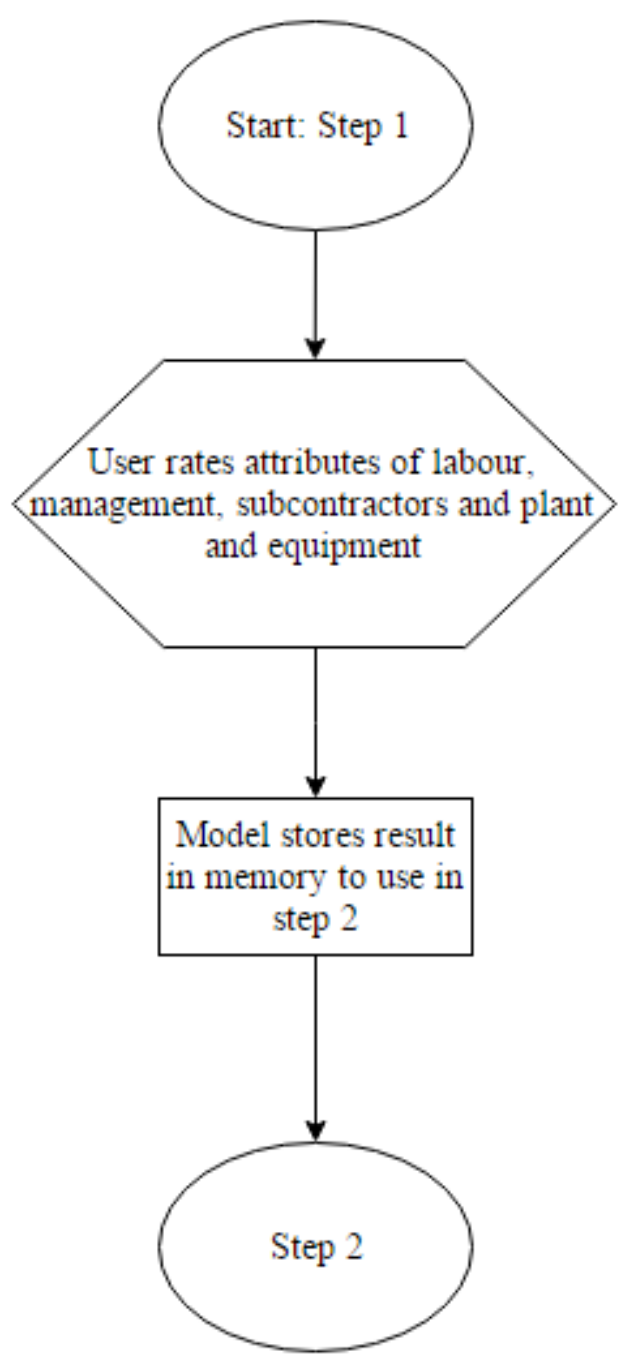

Figure 7.3: Step 1 model logic

From literature, various attributes of "Labour, Management, Subcontractors and Plant and Equipment" have been identified that influence the quality of structural concrete. These are presented in Tables 6.2 to 6.5 in Chapter 6.

In Step 1 of the model, these attributes are rated (0-5) by the contractor for the project under consideration. The input is used to calculate the expected performance of "Labour, Management, Subcontractors and Plant and Equipment." See the partial example for "Labour" in Figure 7.4 where attributes are rated by the contractor.

Table 7.2 provides a list of all the attributes that require input from a user to be able to determine the expected performance of "Labour, Management, Subcontractors and Plant and Equipment."

The user is required to provide a rating between zero and five for all the attributes in Table 7.2. The weights that are shown in Table 7.2 are used in Step 2 of the model. The weights were determined from survey results and are not determined from user input. 
Table 7.2: Performance indicators for each category

\begin{tabular}{|c|c|c|c|c|c|c|c|}
\hline Labour & $\begin{array}{l}\text { Weight(w) } \\
\text { (Step 2) }\end{array}$ & Management & $\begin{array}{c}\text { Weight(w) } \\
\text { (Step 2) }\end{array}$ & Subcontractors & $\begin{array}{l}\text { Weight(w) } \\
\text { (Step 2) }\end{array}$ & $\begin{array}{l}\text { Plant and } \\
\text { equipment }\end{array}$ & $\begin{array}{c}\text { Weight(w) } \\
\text { (Step 2) }\end{array}$ \\
\hline $\begin{array}{l}\text { Ratio of } \\
\text { casual labour }\end{array}$ & 0.195 & Coordination & 0.143 & Communication & 0.235 & $\begin{array}{l}\text { Equipment } \\
\text { standard }\end{array}$ & 0.331 \\
\hline Training & 0.210 & $\begin{array}{l}\text { Precision } \\
\text { planning }\end{array}$ & 0.135 & $\begin{array}{l}\text { Construction } \\
\text { methods }\end{array}$ & 0.245 & $\begin{array}{l}\text { Cleanliness of } \\
\text { formwork, } \\
\text { shutter boards } \\
\text { and } \\
\text { equipment }\end{array}$ & 0.348 \\
\hline Motivation & 0.193 & $\begin{array}{l}\text { Impractical } \\
\text { design }\end{array}$ & 0.137 & $\begin{array}{l}\text { Knowledge on } \\
\text { operations }\end{array}$ & 0.253 & $\begin{array}{l}\text { Machinery } \\
\text { maintenance } \\
\text { and condition }\end{array}$ & 0.322 \\
\hline Skill level & 0.211 & $\begin{array}{l}\text { Quality } \\
\text { management } \\
\text { expertise }\end{array}$ & 0.135 & Experience & 0.267 & - & - \\
\hline Self-discipline & 0.192 & $\begin{array}{l}\text { Quality } \\
\text { management } \\
\text { system }\end{array}$ & 0.141 & - & - & - & - \\
\hline- & - & Supervision & 0.159 & - & - & - & - \\
\hline- & - & Communication & 0.150 & - & - & - & - \\
\hline & $\sum=1.0$ & & $\sum=1.0$ & & $\sum=1.0$ & & $\sum=1.0$ \\
\hline
\end{tabular}

In Figure 7.4 an example is shown where the attributes of labour are given a rating from user input. A rating of zero indicates that extremely poor performance is expected for an attribute. Furthermore, a rating of five indicates that extremely good performance is expected for an attribute.

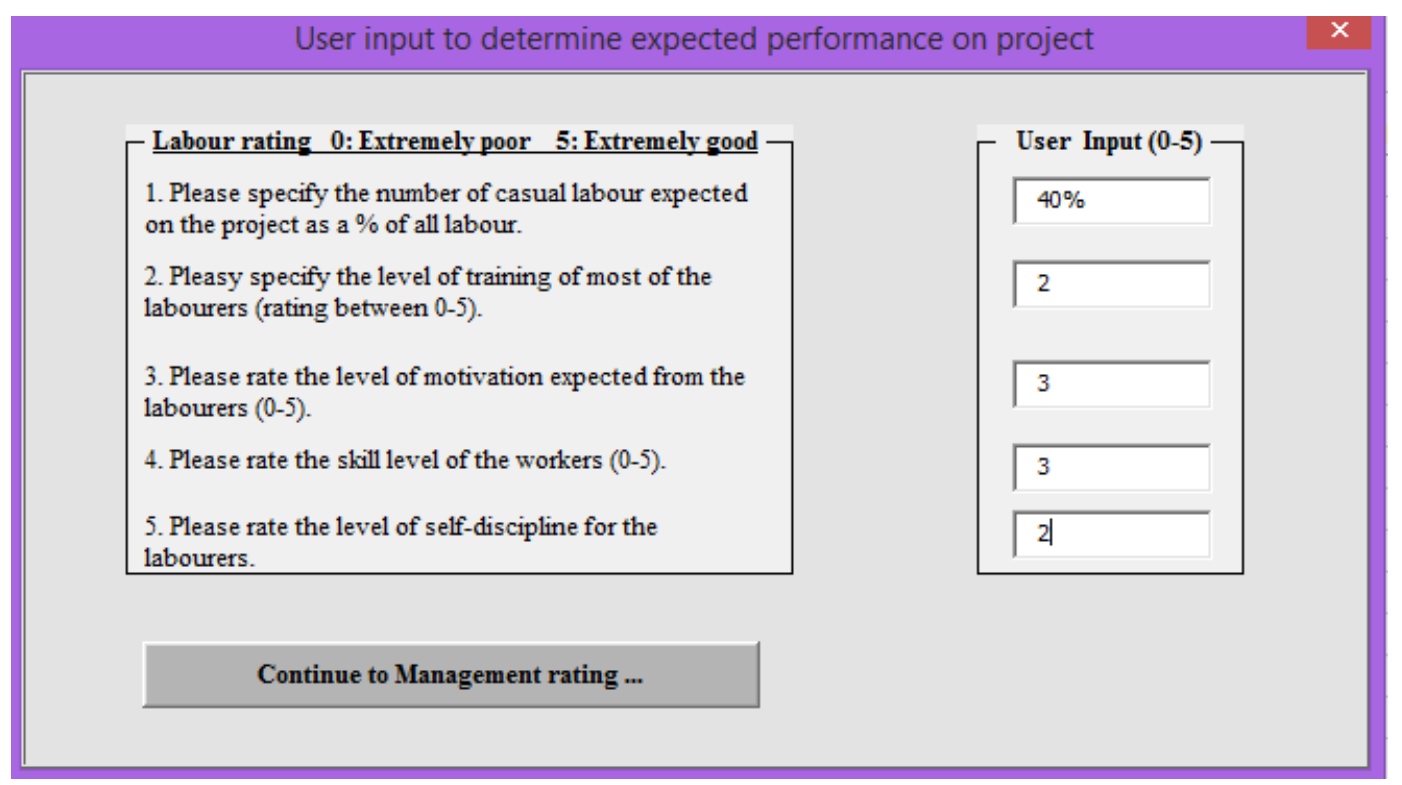

Figure 7.4: Graphical user interface with expected performance from labour as user input

The subsequent step entails the calculation of the category (labour, management, subcontractors and plant and equipment) scores which is done in the background of the model. 


\subsection{Step 2: Category scores}

The diagram in Figure 7.5 shows a brief outline of Step 2 relative to the other steps in the model.

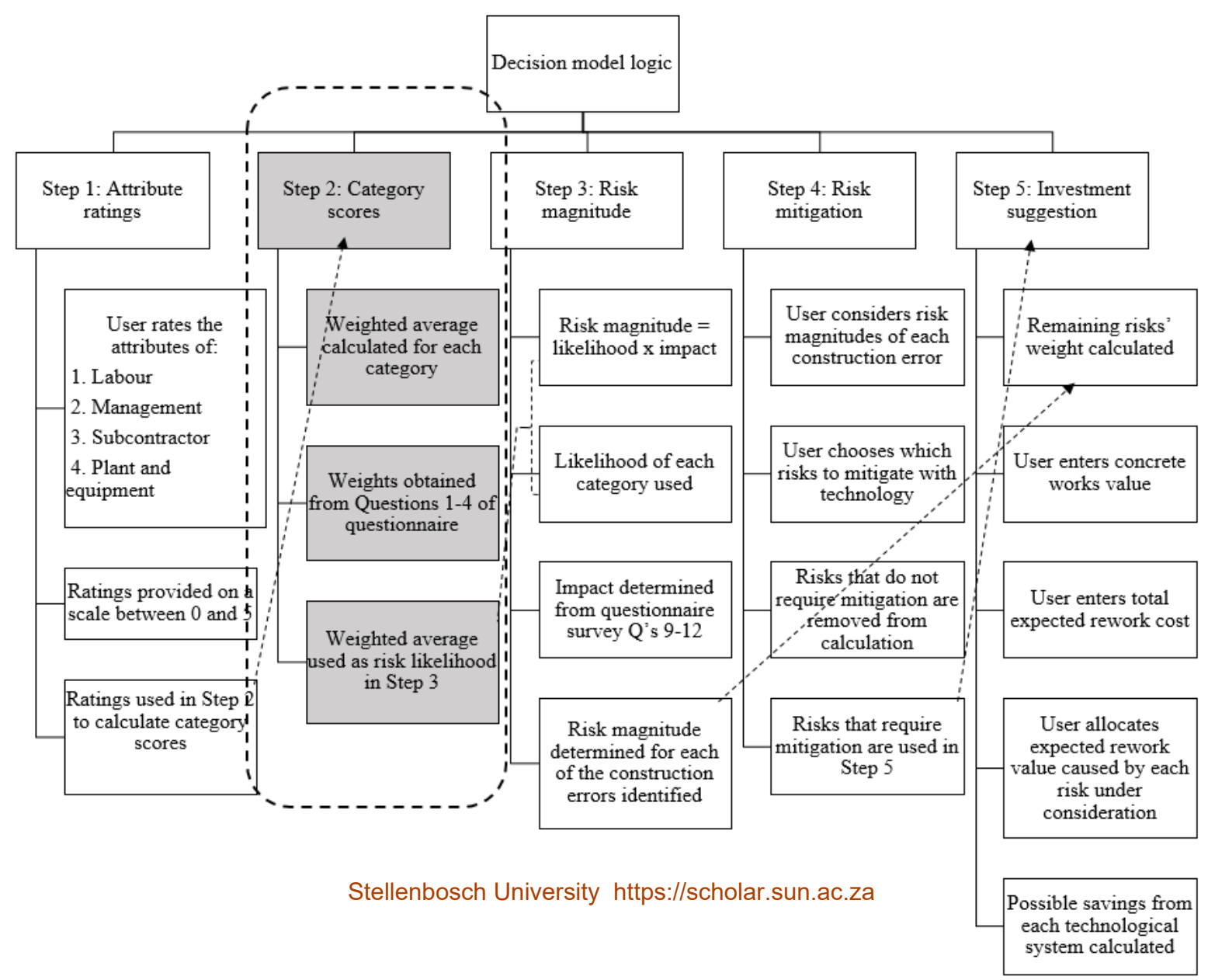

Figure 7.5: Overview of Step 2 in the decision model

The diagram in Figure 7.6 shows the logic of the model for Step 2. Recall that the ellipse indicates a start and end node, while the rectangle indicates an action executed by the model. Step 2 requires no user input to proceed to Step 3. 


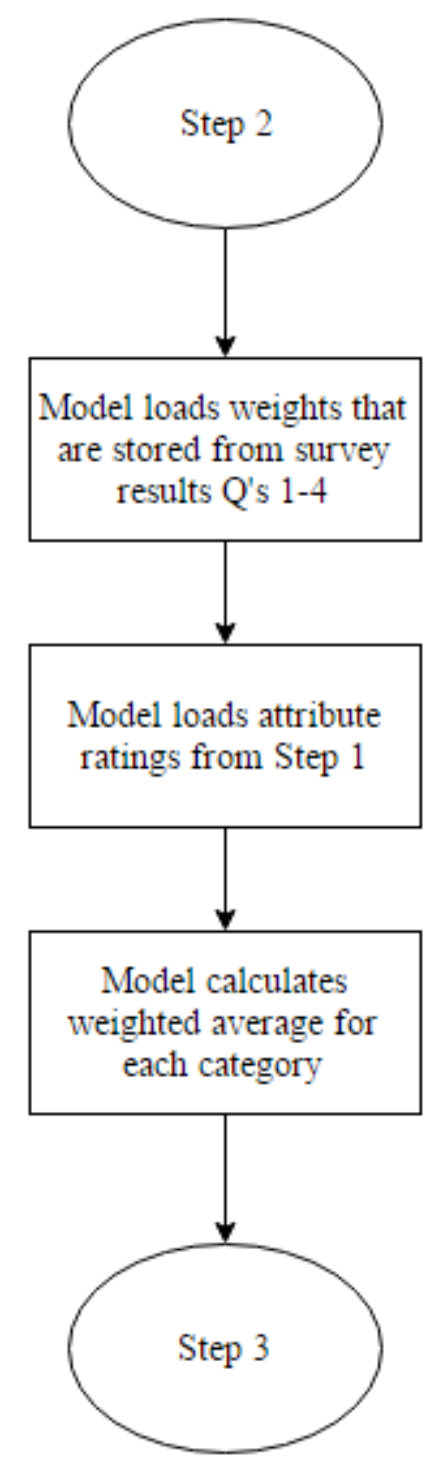

Figure 7.6: Step 2 model logic

The user input obtained from Step 1 is stored in the model. The model then calculates the weighted average of the expected performance for each category(labour, management, subcontractor, plant and equipment) by summing the weighted contribution of each attribute in a category. Each of the attributes that are rated by a user, has a pre-determined weight(shown in results of Questions 1-4 of questionnaire survey). The weights ensure that the model unevenly allocates the influence of each of the attributes on quality. The weights are shown in Table 7.2 .

The weighted average for each category (labour, management, subcontractors and plant and equipment) from Step 2 is used as the expected performance from each category. The likelihood value for each category, in the risk magnitude calculation (Step 3), is derived from the expected performance (Step 2).

The calculation of category scores is illustrated by means of the "Labour" example in Table 7.3. The attribute ratings are obtained through user input in Step 1. The assessed 
proportion represents the contribution of an attribute in a category. The sum of all the assessed proportions give the category average (Equation (7.1)). Note that the user input (Step 1) requires a percentage input for the ratio of casual labour. In Table 7.3 the conversion from percentage to a rating out of 5 is shown.

Table 7.3: Example of Step 2 in the model for the labour category

\begin{tabular}{|c|c|c|c|}
\hline Labour attributes & Weight (Survey) & User input Step 1 & Assessed proportion \\
\hline (a) & (b) & (c) & $(\mathrm{d}=\mathrm{b} \times \mathrm{c})$ \\
\hline Ratio of casual labour & 0.195 & $3(5-40 \% \times 5)$ & 0.585 \\
\hline Training & 0.210 & 2 & 0.420 \\
\hline Motivation & 0.193 & 3 & 0.579 \\
\hline Skill level & 0.211 & 3 & 0.633 \\
\hline Self-discipline & 0.192 & 2 & 0.384 \\
\hline $\begin{array}{l}\text { Weighted average (Category } \\
\text { performance) }\end{array}$ & & & $\sum=2.6$ \\
\hline
\end{tabular}

In Table 7.3 the weight of each attribute is multiplied by the rating obtained from user input. This provides the assessed proportion of each attribute. To calculate the "Category" expected performance, Equation (7.1) is used to aggregate the proportion of each attribute in a category.

$$
\text { Category performance }=\sum \text { Assessed proportions }
$$

For the example under consideration, the expected "Labour" category performance aggregates to 2.6 out of 5 . In the model, the "Category performance" is calculated for each of "Labour, Management, Subcontractors, and Plant and Equipment."

\subsection{Step 3: Risk magnitude}

The diagram in Figure 7.7 shows a brief outline of Step 3 relative to the other steps in the model. 


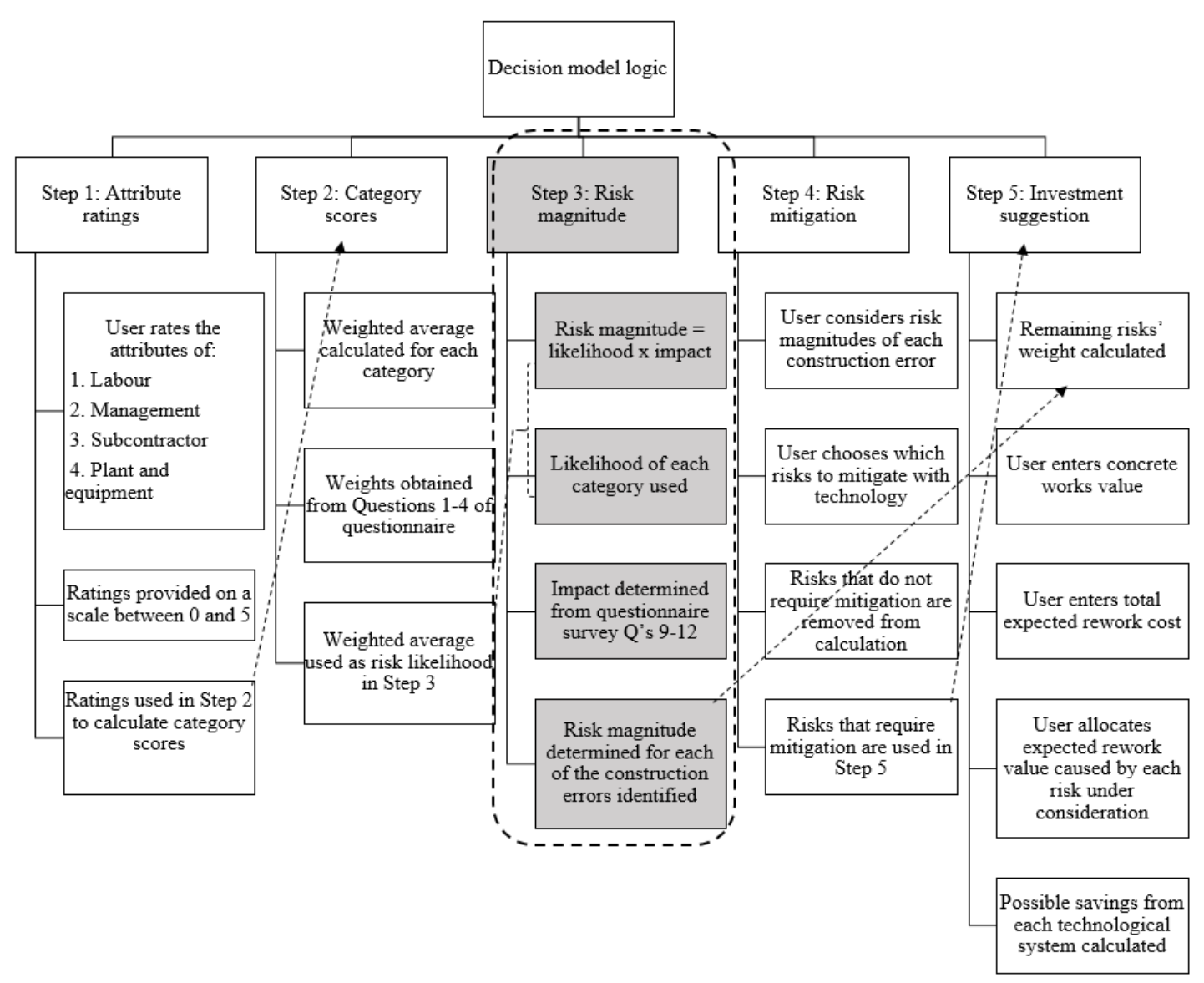

Figure 7.7: Overview of Step 3 in the decision model

The diagram in Figure 7.8 shows the logic of the model for Step 3. Recall that the ellipse indicates a start and end node, while the rectangle indicates an action executed by the model. The process in Figure 7.8 is carried out for each of the risks identified in this study. 


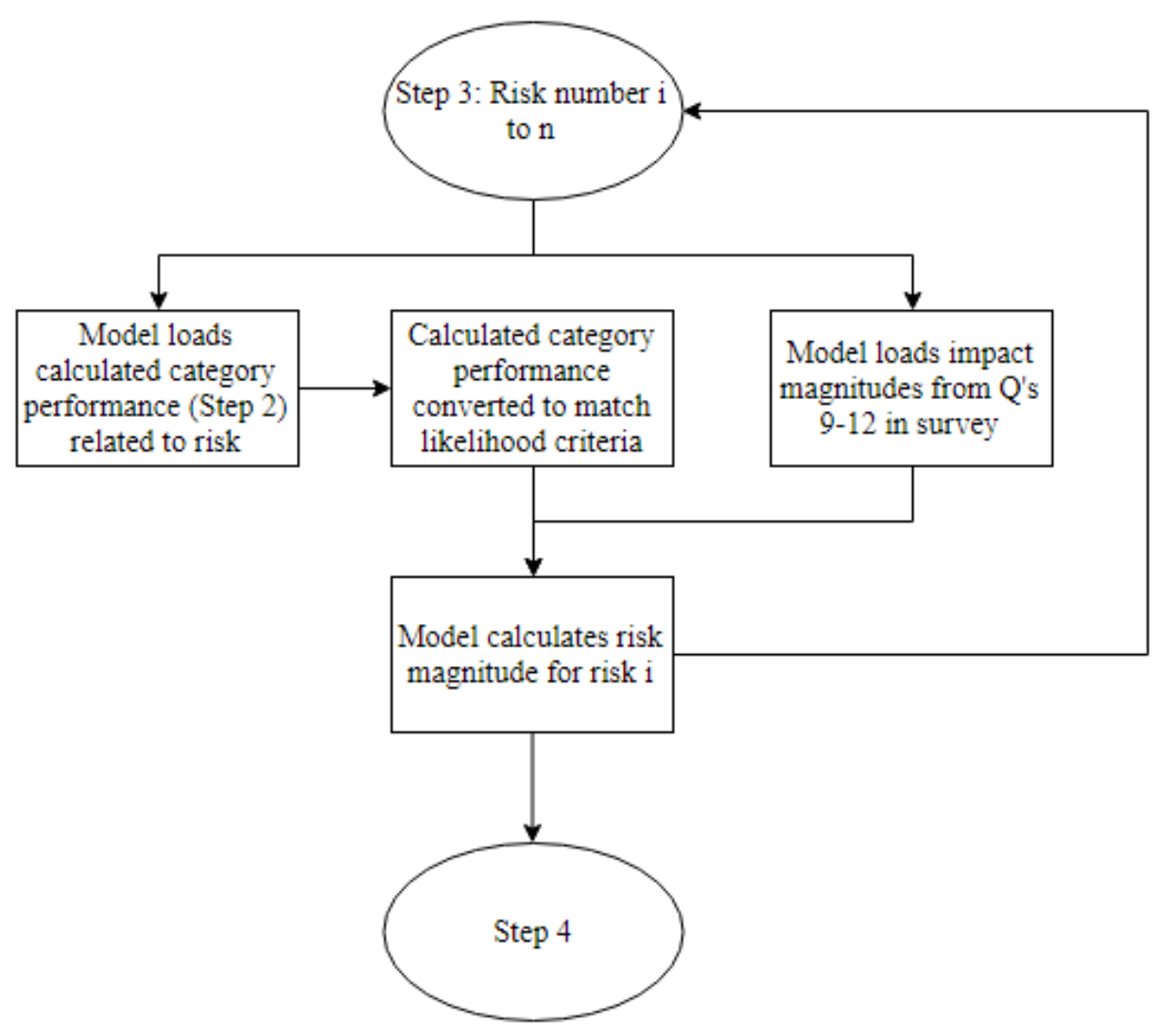

Figure 7.8: Step 3 model logic

A risk matrix is used to determine the magnitude of the risks under consideration. The risks represent the construction errors that lead to rework (see Section 5.1 on $\mathrm{p}$.84). The risk magnitude is determined by means of Equation (7.2).

$$
\text { Risk magnitude }=\text { Likelihood } \times \text { Impact }
$$

The category performance rating (eq. (7.1)) for labour, management, subcontractors, and plant and equipment is stored in the model from Step 2. The rating, with a value out of 5 , represents the expected performance from each category. Since the attributes under each category were determined from literature as factors leading to poor quality, an assumption was made as follows:

- It is assumed that the performance expected from a category is an indication of an associated degree of quality that is likely to result from that category.

The risk matrix contains a likelihood and an impact axis. The calculated category performance (Step 2) represents the input for the likelihood axis.

\subsubsection{Likelihood criteria}

The category performance ratings (Step 2) are converted to fit the likelihood criteria in Table 7.6. Equation (7.3) is used to convert the category rating. It is converted to simplify the manner in which the risk matrix is used in Table 7.6. 


\section{Converted rating $=5-$ Category performance rating}

The likelihood axis of the risk matrix is sub-divided into the categories shown in Table 7.4. Table 7.4 shows that when the expected performance of a category is "Excellent" then the likelihood of poor quality occurring, as a result of the attributes of that category, would be "Very Unlikely." However, the description does not influence the risk magnitude calculation since the numerical values are used.

Table 7.4: Risk likelihood criteria

\begin{tabular}{cll}
\hline Rating score(0-5) & $\begin{array}{l}\text { Expected } \\
\text { performance }\end{array}$ & $\begin{array}{l}\text { Likelihood category } \\
\text { description }\end{array}$ \\
\hline $\mathrm{x} \leq 1$ & Excellent & Very unlikely \\
\hline $1<\mathrm{x} \leq 2$ & Good & Unlikely \\
\hline $2<\mathrm{x} \leq 3$ & Fair & Possible \\
\hline $3<\mathrm{x} \leq 4$ & Poor & Likely \\
\hline $4<\mathrm{x} \leq 5$ & Very poor & Very likely \\
\hline
\end{tabular}

Each of the likelihood descriptions associated with the performance of labour, management, subcontractors, and plant and equipment stays constant throughout the model. Thus, for the various construction errors/risks connected to a single category (labour, management, subcontractors, or plant and equipment) the same likelihood value is used in the risk magnitude calculation. In other words, when considering the risk magnitude of the individual construction errors, the same likelihood description is used for risks that result from the same category (labour, management, subcontractors, and plant and equipment). For example, the likelihood derived from the attribute ratings of labour, is used as the likelihood that the steel spacing, compaction and measures to prevent kicking of formwork, could be incorrect (errors caused by labour).

An example using the "Labour" category is chosen to explain the model.

From Table 7.3 , the category performance rating for labour was 2.6 out of 5 . The rating is converted through Equation $(7.3)$ so that it corresponds to the criteria in Table 7.4. The adjusted rating is to 2.4 out of $5(=5-2.6)$. From Table 7.4 it is seen that the rating lies within the "Fair" expected performance rating. This corresponds to a "Possible" likelihood description in the risk magnitude calculation. The "Possible" likelihood description is used for all the identified construction errors/risks caused by labour.

\subsubsection{Impact criteria}

The impact of each category such as labour, management, subcontractors and, plant and equipment, on the identified construction errors, were determined by means of the questionnaire survey. Note that each category consists of attributes that were previously mentioned in Step 1. The results are shown in Tables 6.7 to 6.10 of Chapter 6. 
The impact ratings are used on the impact axis of the risk matrix. The impact axis of the risk matrix is sub-divided into the criteria as shown in Table 7.5. However, the description does not influence the risk magnitude calculation since the numerical values are used.

Table 7.5: Risk impact criteria

\begin{tabular}{lcc}
\hline $\begin{array}{l}\text { Impact } \\
\text { description }\end{array}$ & $\begin{array}{l}\text { Numerical } \\
\text { conversion }\end{array}$ & Mean range \\
\hline Negligible & 1 & $\mathrm{x}=1$ \\
\hline Minor & 2 & $1<\mathrm{x} \leq 2$ \\
\hline Moderate & 3 & $2<\mathrm{x} \leq 3$ \\
\hline Significant & 4 & $3<\mathrm{x} \leq 4$ \\
\hline Severe & 5 & $4<\mathrm{x} \leq 5$ \\
\hline
\end{tabular}

\subsubsection{Risk magnitude calculation}

The risk magnitude is determined through use of the risk matrix. The risk matrix consists of a likelihood and an impact axis. The product of the likelihood and impact provides the risk magnitude. The risks under consideration are that construction errors could occur due to inadequate performance from labour, management, subcontractors and, plant and equipment. The consequence of these risks is rework.

The risk magnitude is determined for each of the construction activities where construction errors could occur. It does not provide an indication of the severity of rework that could occur. The cost implication of rework caused by a risk will be decided by the user in Step 5 of the model. The risk magnitudes assist the user in identifying which construction activities require improved quality control. Table 7.6 shows the risk matrix.

Table 7.6: Risk matrix used to determine risk magnitude

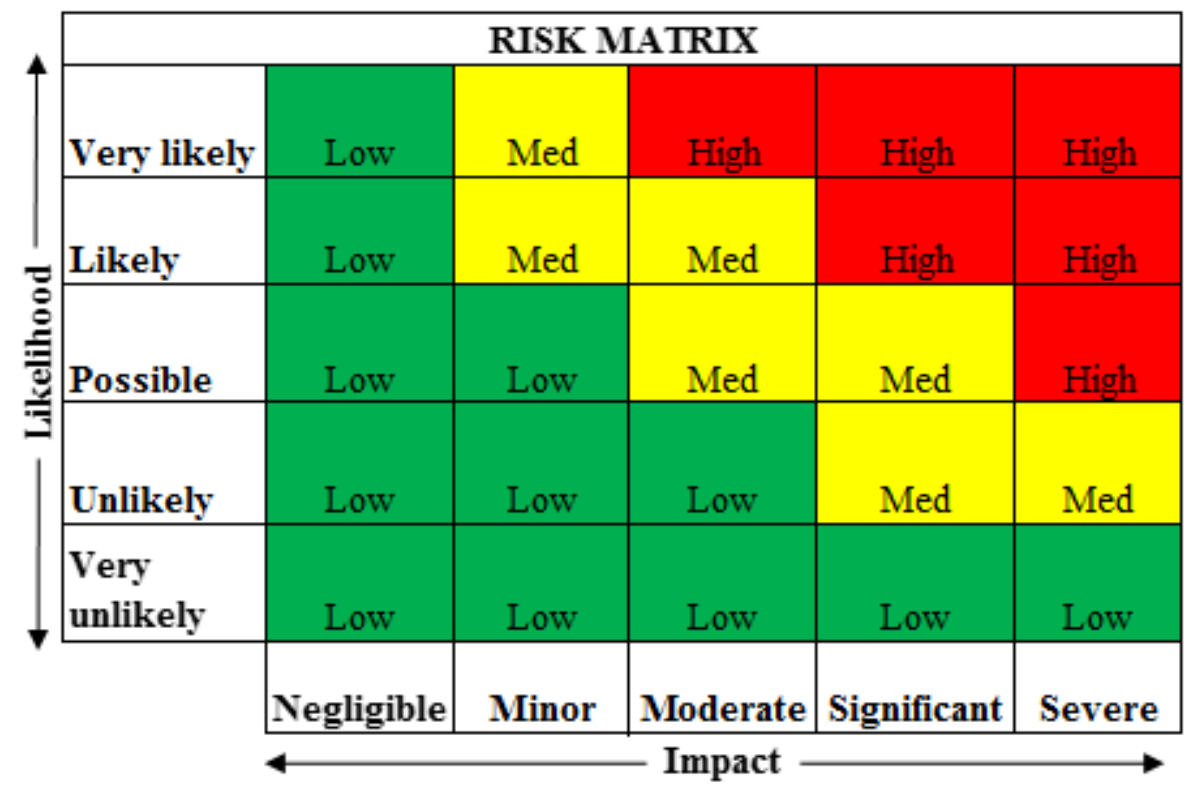


The risk magnitudes are allocated according to three levels of risk:

- Low

- Medium

- High

Table 7.7 shows the maximum underlying scores for the risk matrix combinations.

Table 7.7: Risk matrix used to determine risk magnitude

Very likely
Likely
Possible
Unlikely
Very unlikely

(5)

\begin{tabular}{|c|c|c|c|c|}
\hline 5 & 10 & 15 & 20 & 25 \\
\hline 4 & 8 & 12 & 16 & 20 \\
\hline 3 & 6 & 9 & 12 & 15 \\
\hline 2 & 4 & 6 & 8 & 10 \\
\hline 1 & 2 & 3 & 4 & 5 \\
\hline
\end{tabular}

(1)

(3)

(4)

(5)

Negligible Minor Moderate Significant Severe

The scores are determined by multiplying each likelihood value with the corresponding impact value. The lowest possible combination of likelihood and impact to provide a risk score is $\mathrm{x}=0$ and the highest possible risk score is $\mathrm{x}=25$. A score of $\mathrm{x}<1$ is possible for the likelihood axis in the event that the user provides a zero rating for a number of the attributes in Step 1 (since the attribute ratings from Step 1 determine the likelihood values).

The high risk criteria includes construction errors with a likelihood ranging from "Possible" to "Very likely" and impact ranging from "Moderate" to "Severe." The medium risk criteria includes construction errors with a likelihood ranging from "Very likely" to "Unlikely" and impact ranging from "Minor" to "Severe." It is advised that mitigation measures not be used for risks classified as "Low", but the user makes the ultimate decision. This will be shown in Chapter 8 through application of the model.

In Table 7.7 the axis values that are shown in brackets, represent the maximum rating for each criteria. For example, the likelihood axis has a criteria with description "Very Unlikely" and a score of 1 . The value of 1 represents the maximum rating for a likelihood to be classified as "Very Unlikely." Likelihood values that are smaller than 1 are also classified as "Very Unlikely." Table 7.8 shows the risk magnitude corresponding with a the risk criteria.

Table 7.8: Risk matrix underlying magnitude values

\begin{tabular}{ll}
\hline Risk criteria & Risk magnitude \\
\hline Low & $0 \leq \mathrm{x} \leq 6$ \\
\hline Medium & $6<\mathrm{x} \leq 12$ \\
\hline High & $12<\mathrm{x} \leq 25$ \\
\hline
\end{tabular}


In the "Labour" example a likelihood rating of 2.4 (=5-2.6 through eq. (7.3)) was obtained and classified as "Possible." When considering only the risk of steel spacing being incorrect, an impact magnitude of 3.80 (Table 6.7 Chapter 6) was obtained for structural elements. This corresponds to the "Significant" description (obtained from survey results). The risk magnitude is calculated as $(=2.4 \times 3.8) 9.12$. This falls within the "Medium" risk criteria; therefore, the risk of incorrect steel spacing occurring is medium for the example under consideration. Risk mitigation measures are thus proposed to improve the quality control over steel spacing. The next Section shows the process of identifying which risks require mitigation.

\subsection{Step 4: Risk mitigation}

The diagram in Figure 7.9 shows a brief outline of Step 4 relative to the other steps in the decision model.

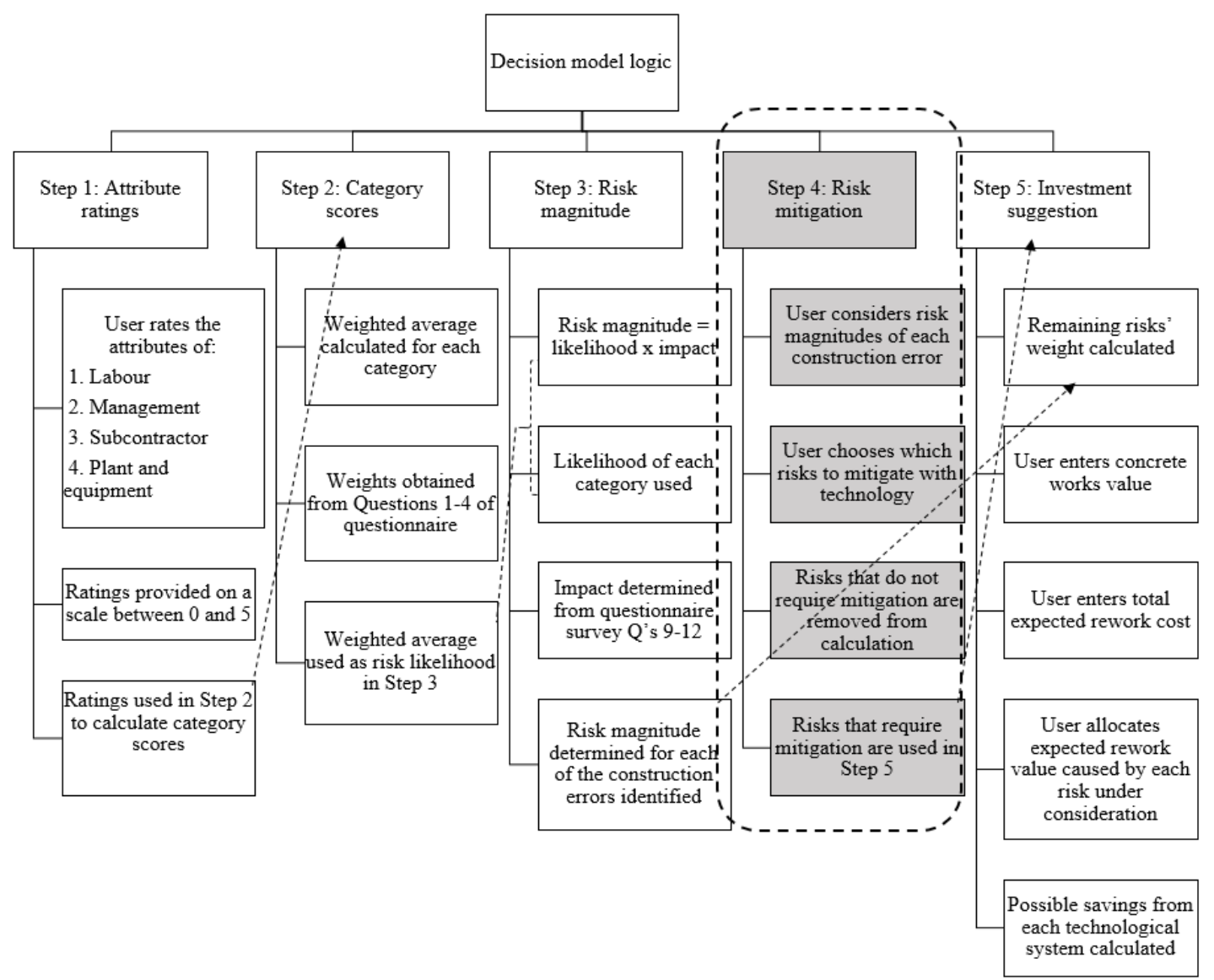

Figure 7.9: Overview of Step 4 in the decision model

The diagram in Figure 7.10 shows the logic of the model for Step 4. Recall that the ellipse indicates a start and end node, while the rectangle indicates an action executed by the model. The diamond shape represents a decision node where the user makes a decision. The process is completed for each of the risks identified in this study. 


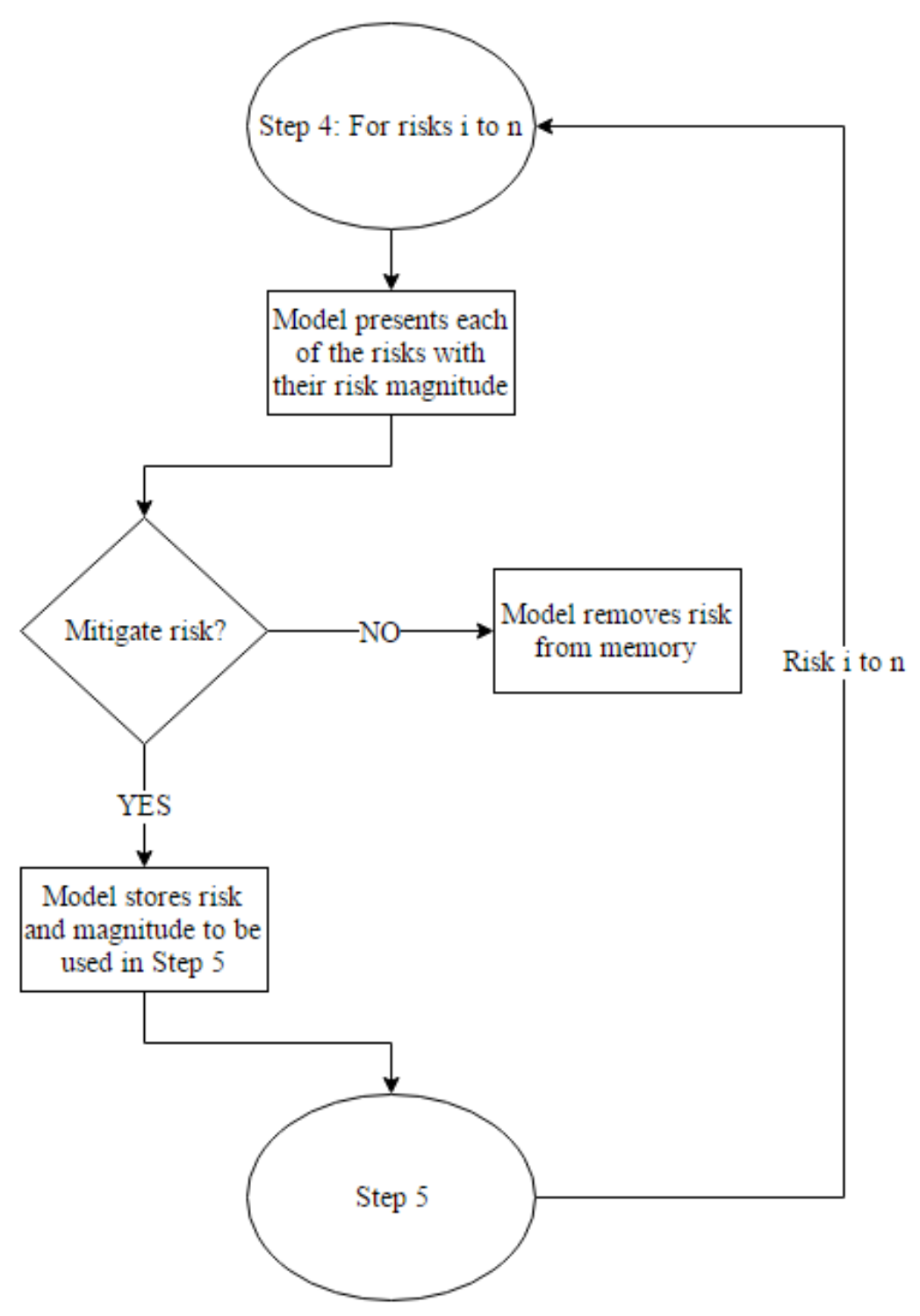

Figure 7.10: Step 4 model logic

In Step 4 the user chooses the risks that require mitigation. The risk magnitudes that were calculated in Step 3 provides the user with an indication of the risks that are likely to occur. However, the user has the opportunity to choose the risks that require mitigation. It is assumed that the user has adequate experience to determine which risks could lead to excessive rework costs.

In the previous example, the risk of incorrect steel spacing was calculated as "Medium." However, let's assume a situation where it was calculated to be "High." The user knows from personal experience that the rework resulting from incorrect steel spacing does not result in excessive rework costs; therefore, the user decides to rather accept the risk instead of mitigating it with technology.

In a different example, the risk of incorrect concrete vibration might be "Medium", but from personal experience the user expects that the risk could lead to excessive rework; therefore, the user decides to mitigate the risk instead of accepting it. The choice of 
mitigation measures (technology) and its effectiveness to mitigate the risks is addressed under Step 5.

\subsection{Step 5: Investment suggestion}

The diagram in Figure 7.11 shows a brief outline of Step 5 relative to the other steps in the decision model.

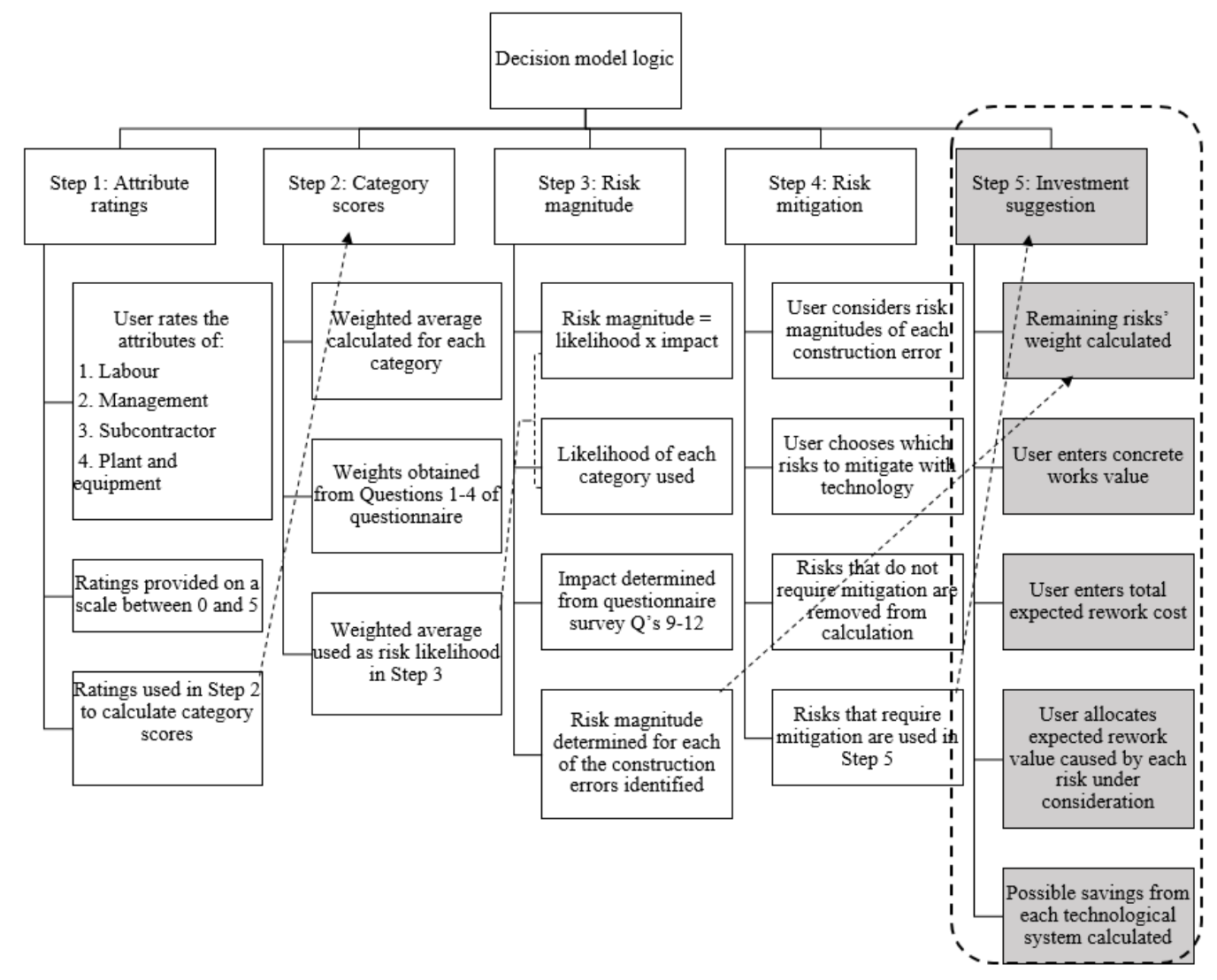

Figure 7.11: Overview of Step 5 in the decision model

The diagram in Figure 7.12 shows the logic of the model for Step 5. Recall that the ellipse indicates a start or end node, while the rectangle indicates an action executed by the model. The hexagon shape represents information required from the user. 


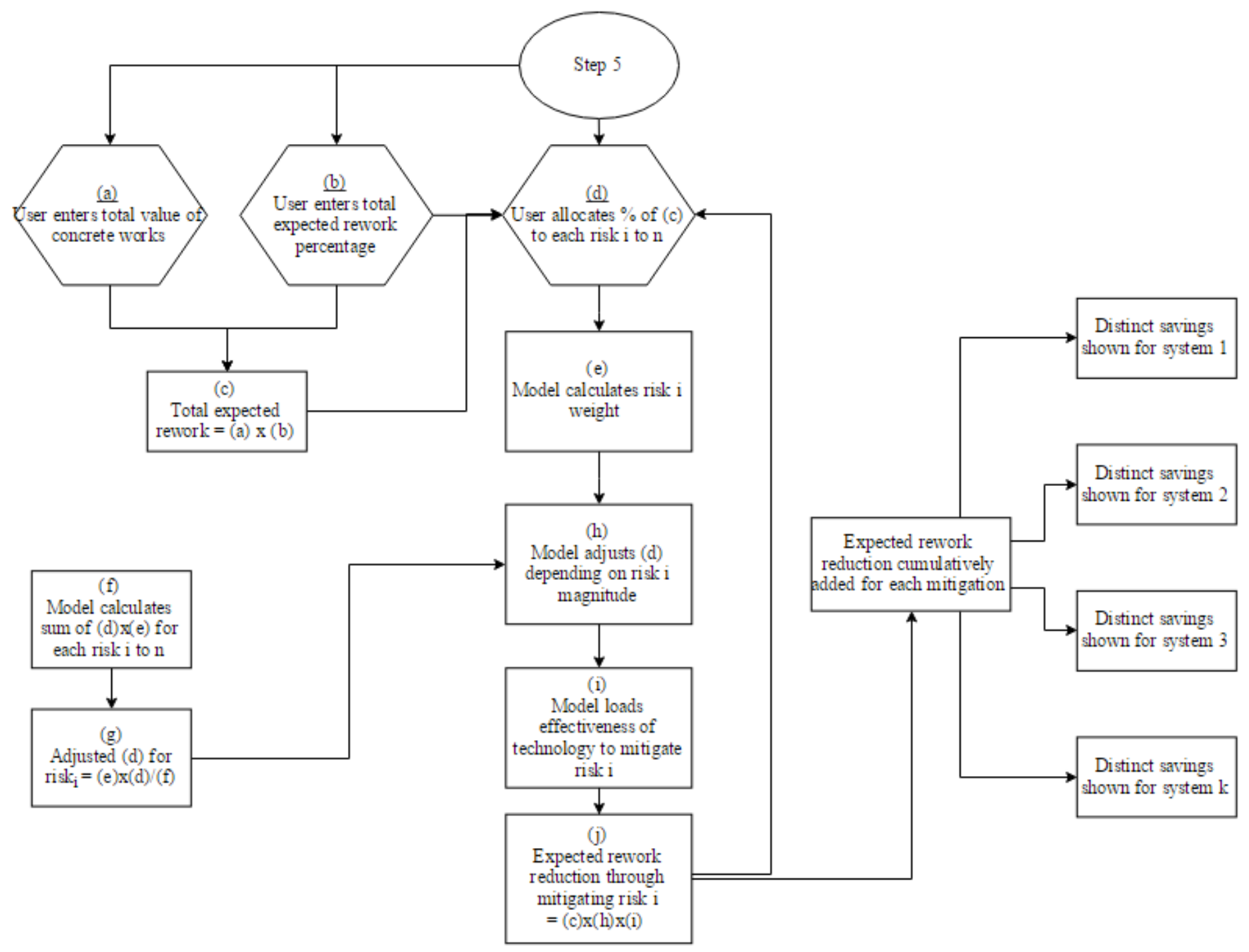

Figure 7.12: Step 5 model logic

The diagram in Figure 7.12 is now explained to provide an understanding of Step 5.

At node (a) the user enters the total value of concrete works on the project. In Step 4 the user chose the risks that require mitigation. At node (b) the user enters the expected value of rework caused by these risks, as a percentage of the total value of concrete works. At node (c) the model calculates the value of the total expected rework by multiplying the total value of concrete works (a) with the expected rework percentage(b).

At node (d) the user allocates a proportion of the total expected rework (c) to each of the risks that require mitigation. This is done since the rework expected from each risk is expected to be uneven; therefore, some risks have the potential to lead to higher rework costs than other. The user does the allocation according to personal experience.

The model previously calculated the risk magnitudes in Step 3 and stored it in the computer memory. At the node (e) the model calculates the weight of each of the remaining 
risks through a normalisation calculation presented in Table 7.9. The rework percentage allocation for each risk, obtained from the user at node (d), are adjusted to account for the risk magnitude of each risk. The adjustment is made through Equation (7.5). The adjustment automatically increases the rework percentage allocation for risks with greater magnitudes and reduces the rework percentage allocation for risks with lower magnitudes.

The effectiveness of the technological systems to mitigate each of the risks are presented in Chapter 5. These values are used to reduce the potential rework savings that could result from mitigating the risks. At node $(\mathrm{j})$ the potential rework saving is calculated for each of the risks by multiplying the total expected rework (c) with the adjusted rework percentage allocation $(\mathrm{h})$ and the effectiveness of the technology. Equation (7.6) also shows the formula. The potential rework saving for each risk is accumulated for each technological system. The model then calculates the savings from using a technological system and compares it to the cost of the system. If the savings exceed the cost of the system then the model proposes it as a feasible investment.

An example, separate from those previously shown in this Chapter, is presented below to demonstrate Step 5 of the model. In Table 7.9 it is assumed that the preceding steps of the model have been completed for the example. Fictitious values are deliberately used in Table 7.9 to demonstrate Step 5 of the model. Therefore, risk classes and risk weights are shown in Table 7.9. The risk weights are calculated as required by node (e) in Figure 7.12. It is assumed that the risks corresponding to the technology effectiveness stated as "N/A" have been chosen by the user in Step 4 as risks that do not require mitigation. Furthermore, the technological mitigating solution is shown for each of the construction risks that require mitigation.

Table 7.9: Construction errors and technological mitigation solutions for the identified risks

\begin{tabular}{|c|c|c|c|c|}
\hline $\begin{array}{l}\text { Construction } \\
\text { error to } \\
\text { monitor }\end{array}$ & $\begin{array}{l}\text { Risk class } \\
\text { (Table } 7.8\end{array}$ & $\begin{array}{c}\text { Risk weight } \\
\left(w_{i}=\right. \\
\left.r_{i} \div \sum r\right)\end{array}$ & $\begin{array}{l}\text { Technological } \\
\text { system } \\
\text { (Available for } \\
\text { mitigation) }\end{array}$ & $\begin{array}{c}\text { Technology } \\
\text { effective- } \\
\text { ness } \\
e_{i}(\%)\end{array}$ \\
\hline Steel spacing & Low & $\mathrm{N} / \mathrm{A}$ & Accept risk & $\mathrm{N} / \mathrm{A}$ \\
\hline Compaction & Medium & 0.05 & IP camera & 55 \\
\hline Grout loss & High & 0.10 & IP camera & 55 \\
\hline $\begin{array}{l}\text { Concrete } \\
\text { cover }\end{array}$ & Medium & 0.05 & Laser scanner & 57 \\
\hline $\begin{array}{l}\text { Kicking of } \\
\text { formwork }\end{array}$ & Low & $\mathrm{N} / \mathrm{A}$ & Accept risk & $\mathrm{N} / \mathrm{A}$ \\
\hline Bleeding & Low & $\mathrm{N} / \mathrm{A}$ & Accept risk & $\mathrm{N} / \mathrm{A}$ \\
\hline $\begin{array}{l}\text { Consistency } \\
\text { of mix }\end{array}$ & High & 0.1 & IDS & 72 \\
\hline $\begin{array}{l}\text { Moisture } \\
\text { condition } \\
\text { during curing }\end{array}$ & High & 0.2 & $\begin{array}{l}\text { Concrete } \\
\text { sensors }\end{array}$ & 73 \\
\hline
\end{tabular}




\begin{tabular}{lcclc}
\hline $\begin{array}{l}\text { Curing } \\
\text { process }\end{array}$ & Medium & 0.06 & IP camera & 55 \\
\hline Lap-lengths & Medium & 0.04 & IDS & 72 \\
\hline Pull-out bars & High & 0.25 & Laser scanner & 57 \\
\hline $\begin{array}{l}\text { Identification } \\
\text { of formwork } \\
\text { and } \\
\text { shuttering } \\
\text { condition }\end{array}$ & Low & N $/ \mathrm{A}$ & Accept risk & N/A \\
\hline $\begin{array}{l}\text { Cleanliness of } \\
\text { equipment } \\
\text { and } \\
\text { formwork }\end{array}$ & Medium & 0.04 & IP camera & 55 \\
\hline $\begin{array}{l}\text { Material } \\
\text { handling }\end{array}$ & Low & N $/ \mathrm{A}$ & Accept risk & N/A \\
\hline $\begin{array}{l}\text { Dimensions } \\
\text { and } \\
\text { alignment of } \\
\text { formwork }\end{array}$ & Medium & 0.05 & Laser scanner & 57 \\
\hline $\begin{array}{l}\text { Position of } \\
\text { HD bolts }\end{array}$ & Medium & 0.06 & Laser scanner & 57 \\
\hline
\end{tabular}

The example in Table 7.9 continues in Table 7.10. Table 7.10 shows the remaining risks after removing the risks that do not require mitigation. It also shows an example of the allocated rework to each risk as a percentage of the total expected rework cost. This would be allocated by the user in a real-life project. This is required at node (d) of the diagram in Figure 7.12 .

Since the user provides these values based on previous experiences, the risk magnitude does not influence the user's decision of the rework allocation. However, the rework allocation for each risk is adjusted using Equation (7.5) to include the effect of the risk magnitude.

Table 7.10: Example continued from Table 7.9 where user provides expected rework costs for each of the risks that require mitigation

\begin{tabular}{lcc}
\hline $\begin{array}{l}\text { Construction error to } \\
\text { monitor }\end{array}$ & $\begin{array}{c}\text { Risk class } \\
(\text { Table } 7.8)\end{array}$ & $\begin{array}{c}\text { Portion of expected } \\
\text { rework allocated } \\
(\%)\end{array}$ \\
\hline Compaction & Medium & 25 \\
\hline Grout loss & High & 3 \\
\hline Concrete cover & Medium & 15 \\
\hline Consistency of mix & High & 10 \\
\hline $\begin{array}{l}\text { Moisture condition during } \\
\text { curing }\end{array}$ & High & 10 \\
\hline Curing process & Medium & 5 \\
\hline Lap-lengths & Medium & 5 \\
\hline
\end{tabular}




\begin{tabular}{lcc}
\hline Pull-out bars & High & 5 \\
\hline $\begin{array}{l}\text { Cleanliness of equipment } \\
\text { and formwork }\end{array}$ & Medium & 2 \\
\hline $\begin{array}{l}\text { Dimensions and alignment } \\
\text { of formwork }\end{array}$ & Medium & 10 \\
\hline Position of HD bolts & Medium & 10 \\
\hline & & $\sum=100 \%$
\end{tabular}

The risk weights (calculated in Table 7.9 ) are used to adjust the rework allocation percentage of each risk in Table 7.10. This is done so that risks with higher magnitudes are adjusted to have higher rework costs. To achieve this for each risk, the portion of expected rework as allocated in Table 7.10 , is multiplied with the corresponding risk weight. These values are normalised by dividing each adjusted value by the total sum of the adjusted values. The adjustment is made through Equations (7.4) and (7.5). This corresponds with node (h) of Figure 7.12 .

$$
X=\sum_{i=1}^{n} \text { Allocated } \% \times w_{i}
$$

$n=$ number of risks that were chosen for mitigation in Step 4

$w_{i}=$ risk weight

$$
w_{j}=\text { Allocated } \% \times w_{i} \div X
$$

The formula to calculate the expected rework reduction for each risk is shown by equation (7.6). The weight $\left(w_{j}\right)$ from Equation (7.5), for each risk, is multiplied by the effectiveness value $\left(e_{i}\right)$ of the technology that is used to mitigate the risk. This value is then multiplied with the total expected rework value $(\mathrm{R})$ to estimate the rework reduced from using the mitigating technology. Equation (7.6) shows the calculation formula. This corresponds with node (j) of Figure 7.12. The example continues in Table 7.11 to demonstrate the process of adjusting the rework allocation and calculating the potential rework saved.

$$
\text { Expected rework reduction } \text { risk }_{i}=w_{j} \times e_{i} \times \text { Total rework expected }(R)
$$

Table 7.11 illustrates Equations $(7.5)$ and $(7.6)$ on the example continued from Table 7.9 on $\mathrm{p}$ 137. For the example it was assumed that the total value of concrete works is $\mathrm{R}$ 100-million. This is required at node (a) as shown in Figure 7.12, At node (b) it is assumed that the total expected rework percentage possibly caused by the risks that require mitigation is $1 \%$. This results in a total expected rework value of $\mathrm{R} 1$-million as required at node (c). 
Table 7.11: Example where the allocated\% of rework costs are adjusted to include effects of the risk magnitudes

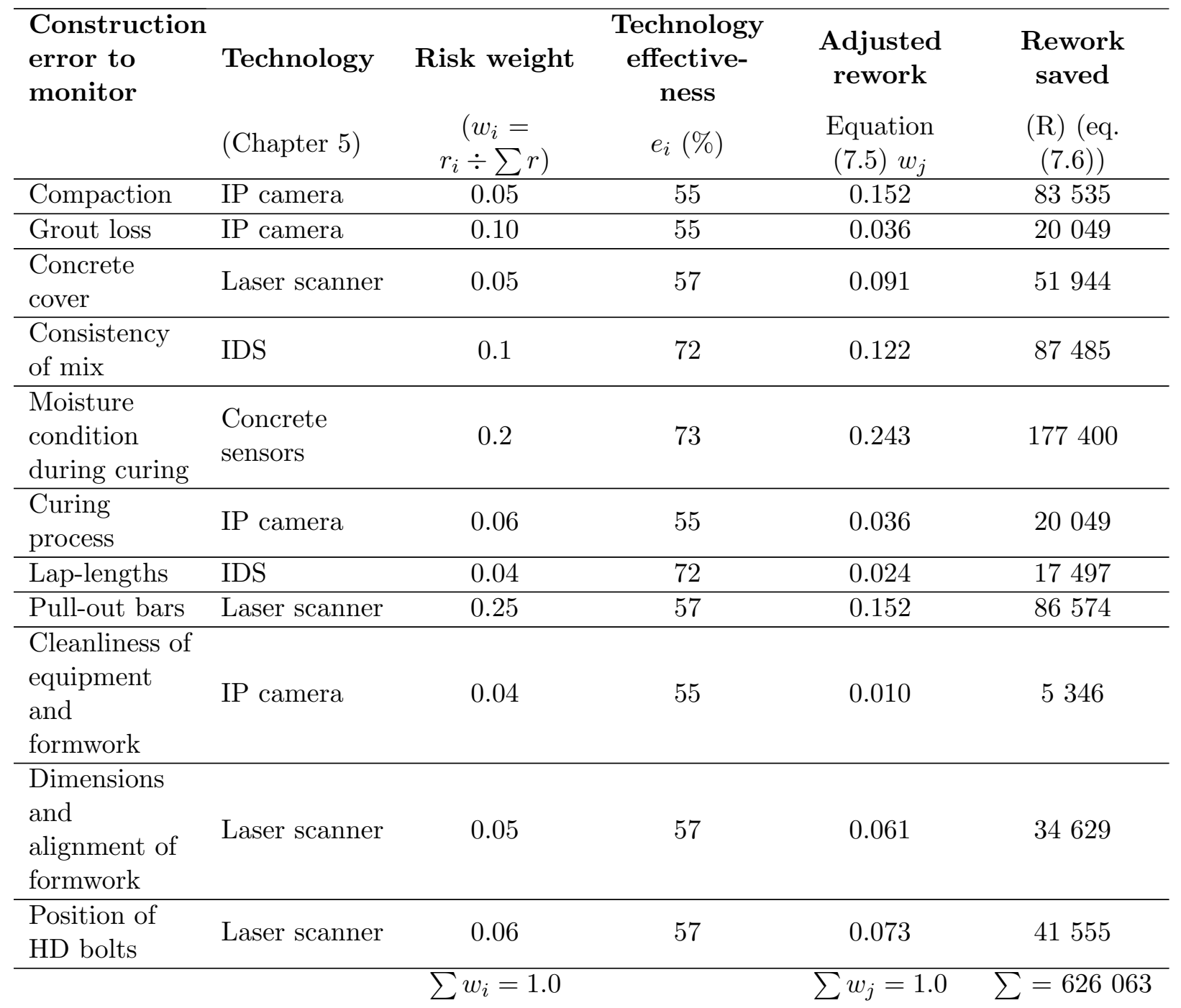

The next step is to determine the potential funds to be invested in each technological system. This is achieved by grouping the risks according to the technology that is used to mitigate them and summing the potential rework savings from that technological system. The cost of the technology is then subtracted from the potential rework savings amount to give the net saving.

Table 7.12 shows the expected net savings for the IP camera. The technological costs are presented in Appendix D. 
Table 7.12: Potential rework saved by using IP camera

\begin{tabular}{lccc}
\hline Construction risk & $\begin{array}{l}\text { Mitigating } \\
\text { technology }\end{array}$ & Rework saved (R) & Net savings (R) \\
\hline Incorrect compaction & IP camera & 83535 & - \\
\hline Grout loss & IP camera & 20049 & - \\
\hline $\begin{array}{l}\text { Incorrect curing } \\
\text { process }\end{array}$ & IP camera & 20049 & - \\
\hline $\begin{array}{l}\text { Inadequate } \\
\text { cleanliness of } \\
\begin{array}{l}\text { equipment and } \\
\text { formwork }\end{array}\end{array}$ & IP camera & 5346 & - \\
\hline$\sum T \operatorname{Total}(R)$ & & 128979 & 98979 \\
\hline \hline
\end{tabular}

In Table 7.12 it is shown that by using the IP camera to mitigate identified construction risks, net savings of R 98979.00 could be achieved. The same process is followed for the image dimensioning software (IDS). Table 7.13 shows the net savings when using IDS.

Table 7.13: Potential rework saved by using IDS in conjunction with logging software

\begin{tabular}{lccc}
\hline Construction risk & $\begin{array}{c}\text { Mitigating } \\
\text { technology }\end{array}$ & Rework saved (R) & Net savings (R) \\
\hline $\begin{array}{l}\text { Incorrect consistency } \\
\text { of mix }\end{array}$ & IDS & 87485 & - \\
\hline Incorrect lap-lengths & IDS & 17497 & - \\
\hline$\sum \operatorname{Total}(R)$ & $(46000)$ & 104982 & 58982 \\
\hline \hline
\end{tabular}

In Table 7.13 it is shown that a potential net saving of R 58982.00 is possible when using the IDS to monitor the identified construction activities. The same process is applied for the laser scanner. Table 7.14 shows the net savings potential when using the laser scanner. 
Table 7.14: Potential rework saved by using laser scanner

\begin{tabular}{lccc}
\hline Construction risk & $\begin{array}{c}\text { Mitigating } \\
\text { technology }\end{array}$ & Rework saved (R) & $\begin{array}{c}\text { Net } \\
\text { savings/(Loss) } \\
(\mathbf{R})\end{array}$ \\
\hline $\begin{array}{l}\text { Incorrect concrete } \\
\text { cover }\end{array}$ & Laser scanner & 51944 & - \\
\hline $\begin{array}{l}\text { Pull-out bars } \\
\text { incorrect }\end{array}$ & Laser scanner & 86574 & - \\
\hline $\begin{array}{l}\text { Incorrect dimensions } \\
\text { and alignment }\end{array}$ & Laser scanner & 34629 & - \\
\hline $\begin{array}{l}\text { Incorrect position of } \\
\text { HD bolts }\end{array}$ & Laser scanner & 41555 & $(620298)$ \\
\hline$\sum T \operatorname{Total}(R)$ & $(835000)$ & 214702 & \\
\hline \hline
\end{tabular}

From Table 7.14 it is shown that, for the example under consideration, a net loss of $\mathrm{R} 620$ 298.00 is possible when investing in the laser scanner. The laser scanner is very expensive and might only be worth acquiring if it will be used for a number of projects.

In Table 7.15 the same process is followed for the concrete curing sensors.

Table 7.15: Potential rework saved by using concrete sensors

\begin{tabular}{lccc}
\hline Construction risk & $\begin{array}{c}\text { Mitigating } \\
\text { technology }\end{array}$ & Rework saved (R) & $\begin{array}{c}\text { Net } \\
\text { savings/(Loss) } \\
(\mathbf{R})\end{array}$ \\
\hline $\begin{array}{l}\text { Moisture condition } \\
\text { during curing }\end{array}$ & Concrete sensors & 177400 & 132400 \\
\hline$\sum \operatorname{Total}(R)$ & $(45000)$ & 177400 & 132400 \\
\hline \hline
\end{tabular}

The potential rework reduction that can be realised by using the sensors are R 177400.00 . The cost of the sensor system is R 45 000.00. Therefore, net savings of R 132400.00 is possible when using the concrete curing sensors.

The total realisable savings is calculated as the sum of the net savings. For this example the total net savings are R 290 361.00. This excludes the use of the laser scanner, since the laser scanner will result in a net loss. Table 7.16 shows the calculation of the total net savings. 
Table 7.16: Total net savings from combination of technologies

\begin{tabular}{lccc}
\hline Technology & Technology cost & Rework saved (R) & $\begin{array}{c}\text { Net } \\
\text { savings/(Loss) } \\
(\mathbf{R})\end{array}$ \\
\hline IP camera & $(30000)$ & 128979 & 98979 \\
\hline IDS & $(46000)$ & 104982 & 58982 \\
\hline Laser scanner & - & - & - \\
\hline Concrete sensors & $(45000)$ & 177400 & 132400 \\
\hline$\sum \operatorname{Total}(R)$ & $(121000)$ & 411361 & 290361 \\
\hline \hline
\end{tabular}

Some of the construction activities can be monitored by more than one type of technology. The model will calculate the effect on savings for all the possible technology systems and then provide the user with the most economic proposal.

The advantage of using technology to reduce rework is that expenses are now transformed into assets. As a conservative approach, it is assumed that the technology only lasts for the duration of the project, but realistically it could be used for more than one project. For a subsequent project it would not be necessary for the capital expenditure and the system could be very advantageous towards increasing project profit by reducing rework. 


\section{Chapter 8}

\section{Model validation}

Figure 8.1 shows an overview of the scope of Chapter 8 relative to other Chapters in this study.

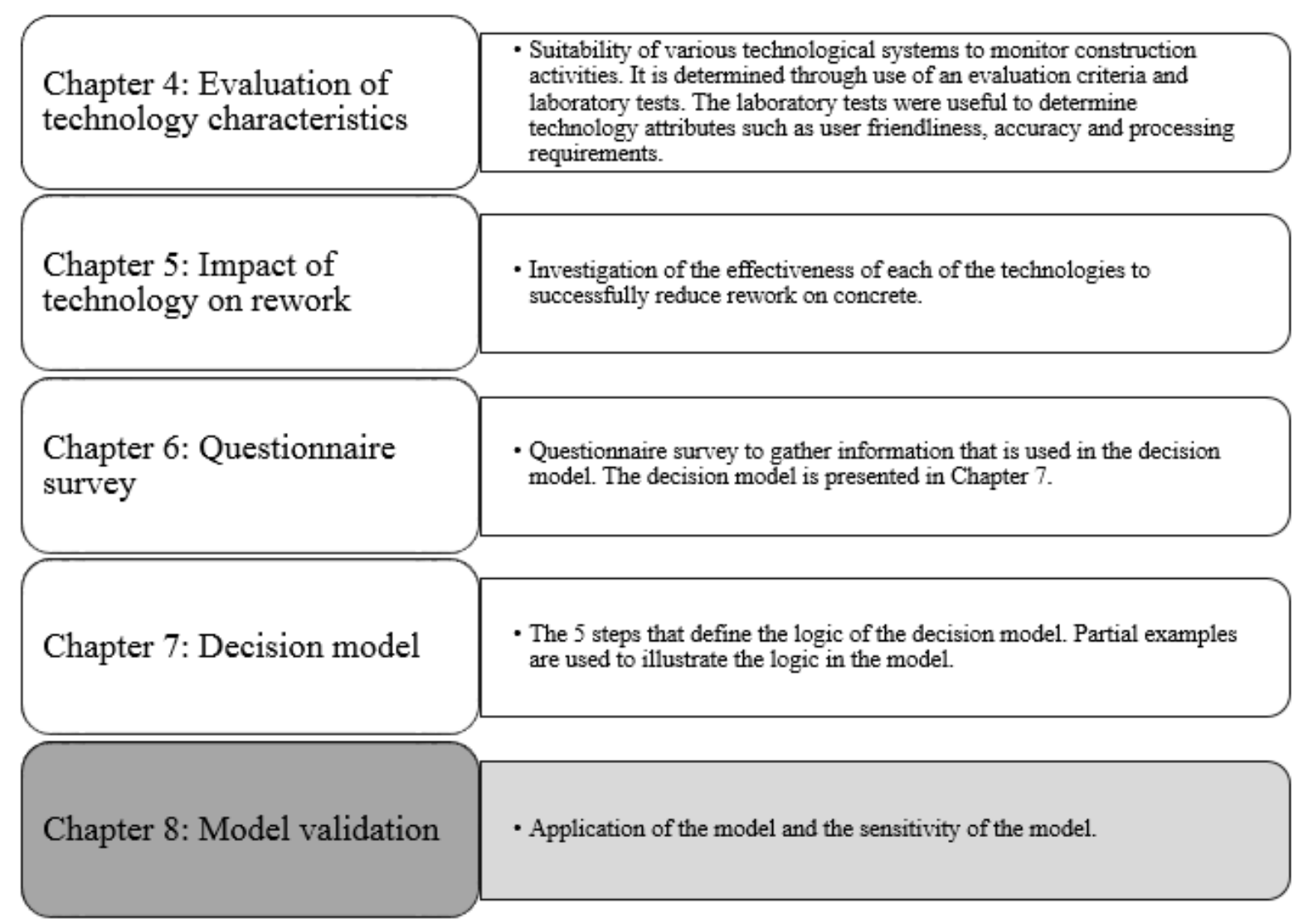

Figure 8.1: Scope of Chapter 8

In this Chapter, the decision model is presented in a usable software format after programming it in "Visual Basic for Applications" (VBA). The model is used to analyse the potential rework reduction for various scenarios of a fictitious project in the Eastern Cape. Furthermore, sensitivity analyses are conducted to determine the model robustness. The model is then presented to industry by means of interviews to gain opinions from industry experts. 


\subsection{Scenario analysis}

To test the model on a real-life project, it is required that the model be applied at the initiation stages of construction. The contractor then needs to invest in the technology and use it over the duration of the project.The accuracy of the model, to predict the potential rework reduction value( $\mathrm{R})$, can then only be determined at the end of the project. Therefore, the model was not tested on a real-life construction project in this study, since the author was limited to a given time span to complete the study.

The author assumed a fictitious project in the Eastern Cape. The project has the following specifications and requirements:

- Three storey building used as a school with $5000 \mathrm{~m}^{2}$ floor area

- Structural concrete to be used as building material for foundations, slabs, columns, beams and specified walls

- At least $50 \%$ of the labourers need to be appointed from the community to support the local economy and skills upliftment

- Value of concrete works is R 100-million

- The allowed construction period is 18 months.

By simulating five fictitious scenarios the author will compare the model output. In the five scenarios the value of concrete works stays constant. The output from the different scenario conditions aims to illustrate the decrease in potential rework savings as the project risk decreases. For the purposes of the simulations, it is assumed that all risks classified as "Low" do not require mitigation. In scenario 1 the author illustrates the use of the model in detail, while in the subsequent scenarios only a summary of the model output is presented.

\subsubsection{Scenario 1: Poor performance expectancy from all cate- gories}

In scenario 1 the performance expected from all the categories are deliberately chosen to be poor. Thus the following information suggests poor performance expectancy for demonstrative purposes.

Performance expectancy from labour:

- The contractor decides to use $70 \%$ casual labour

- The training of all the labourers consist of a one day training course

- The labourers show little motivation to be involved in the construction

- The labourers have very poor skill due to a lack of experience

- The self-discipline of the labourers is low. 
The labour attribute ratings are shown in Figure 8.2. Figures 8.2 to 8.5 represent Step 1 of the model logic as presented in Chapter 7 .

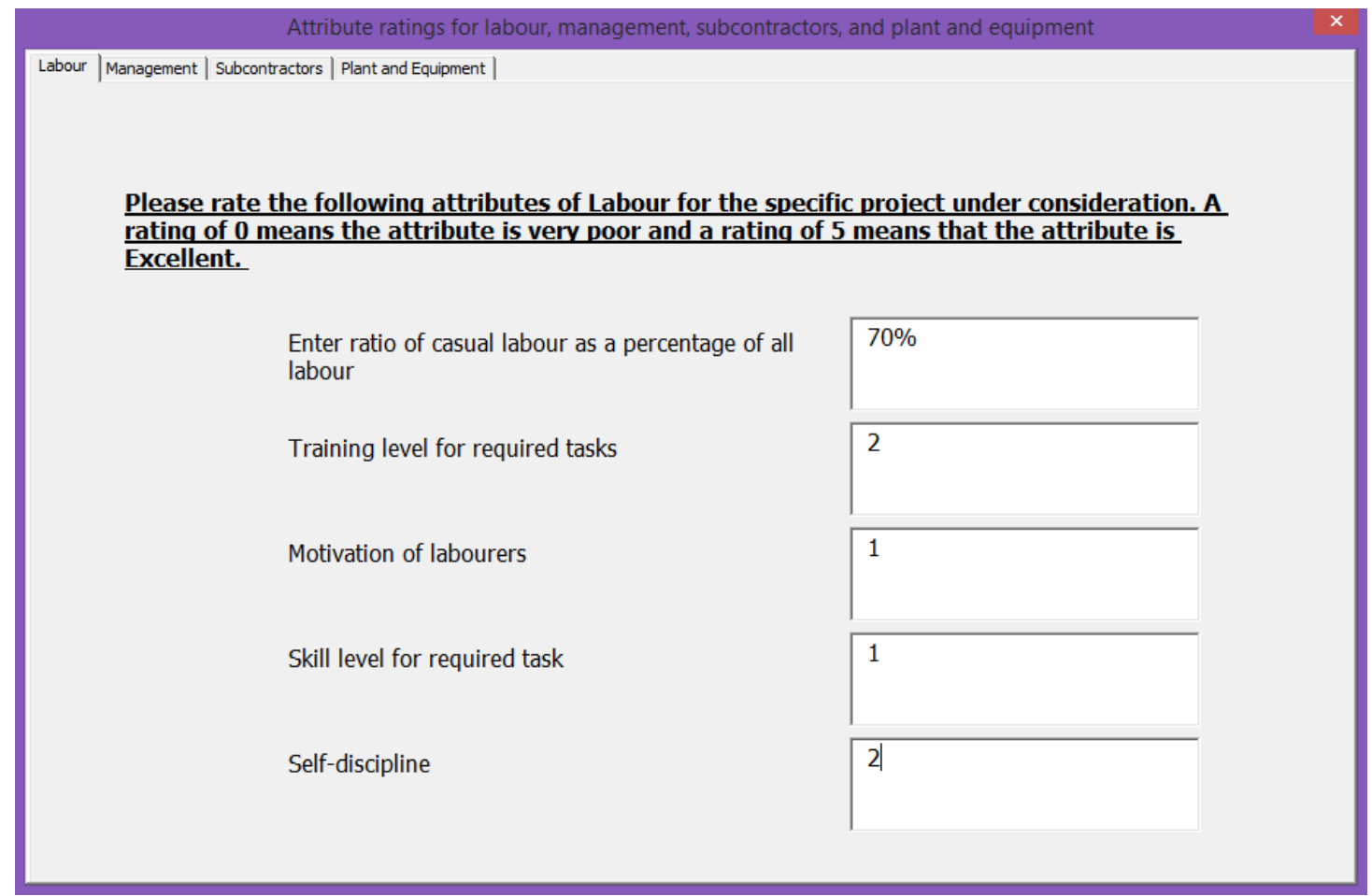

Figure 8.2: Labour attribute ratings for scenario 1

The management team consists of inexperienced site agents and foremen because of a lack of interest by the experienced staff to live in a remote location for 18 months. The performance expectancy from management is deliberately chosen as follows:

- Coordination is expected to be poor

- Project planning not conducted in much detail

- The design by the architect includes a few difficult sections which are impractically designed

- The management team does not have much quality management expertise

- There is no quality management system in place

- Supervision is expected to be poor by the inexperienced managers

- Communication is expected to be difficult because of language differences.

Figure 8.3 shows the input for the management attributes. 


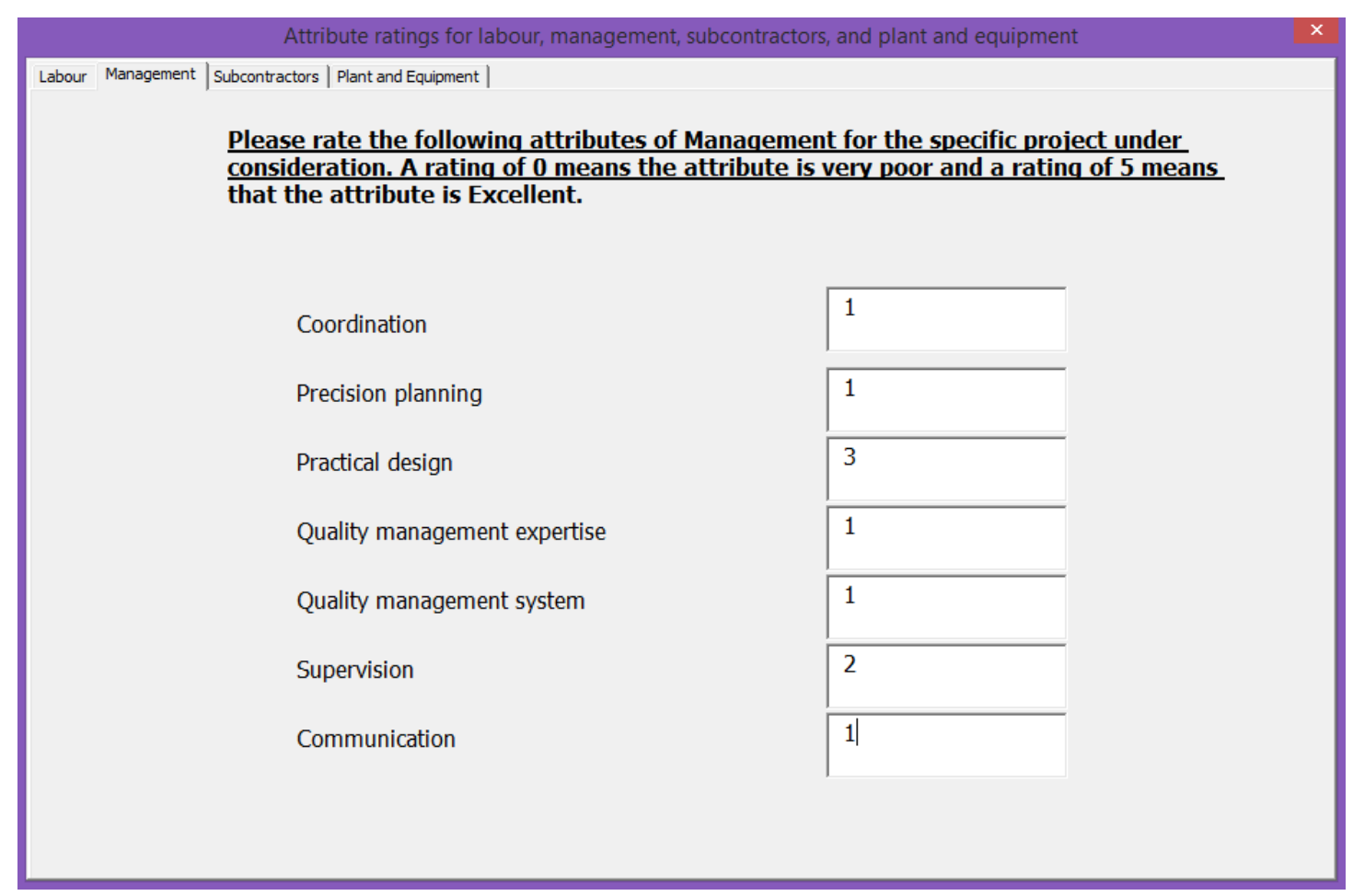

Figure 8.3: Management attribute ratings for scenario 1

Subcontractors from the community are appointed as an initiative to improve the local economy. The subcontractors are primitive; therefore, they do not invest funds toward better equipment and improvement of construction operations. Furthermore, they do not have much experience with triple storey concrete buildings. The performance expectancy of the subcontractors is deliberately chosen as follows:

- Communication is expected to be poor because of language barriers

- Old construction methods are used that are less effective

- The subcontractors have little knowledge of structural elements such as beams and columns

- The subcontractor has little experience with structural concrete works.

Figure 8.4 shows the attribute ratings for the subcontractors. 
Labour | Management Subcontractors | Plant and Equipment |

Please rate the following attributes of Subcontractors for the specific proiect under consideration. A rating of 0 means the attribute is very poor and a rating of 5 means that the attribute is Excellent.

Communication

Construction methods

Knowledge on operations

Experience

\section{1}
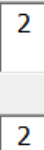

2

Figure 8.4: Subcontractor attribute ratings for scenario 1

The plant and equipment used by the contractor and subcontractors are deliberately defined as follows:

- Poor standard-, damaged- and old equipment

- Cleaning of equipment not conducted at regular intervals

- Machinery not maintained at recurring intervals.

Figure 8.5 shows the attribute ratings for plant and equipment. 


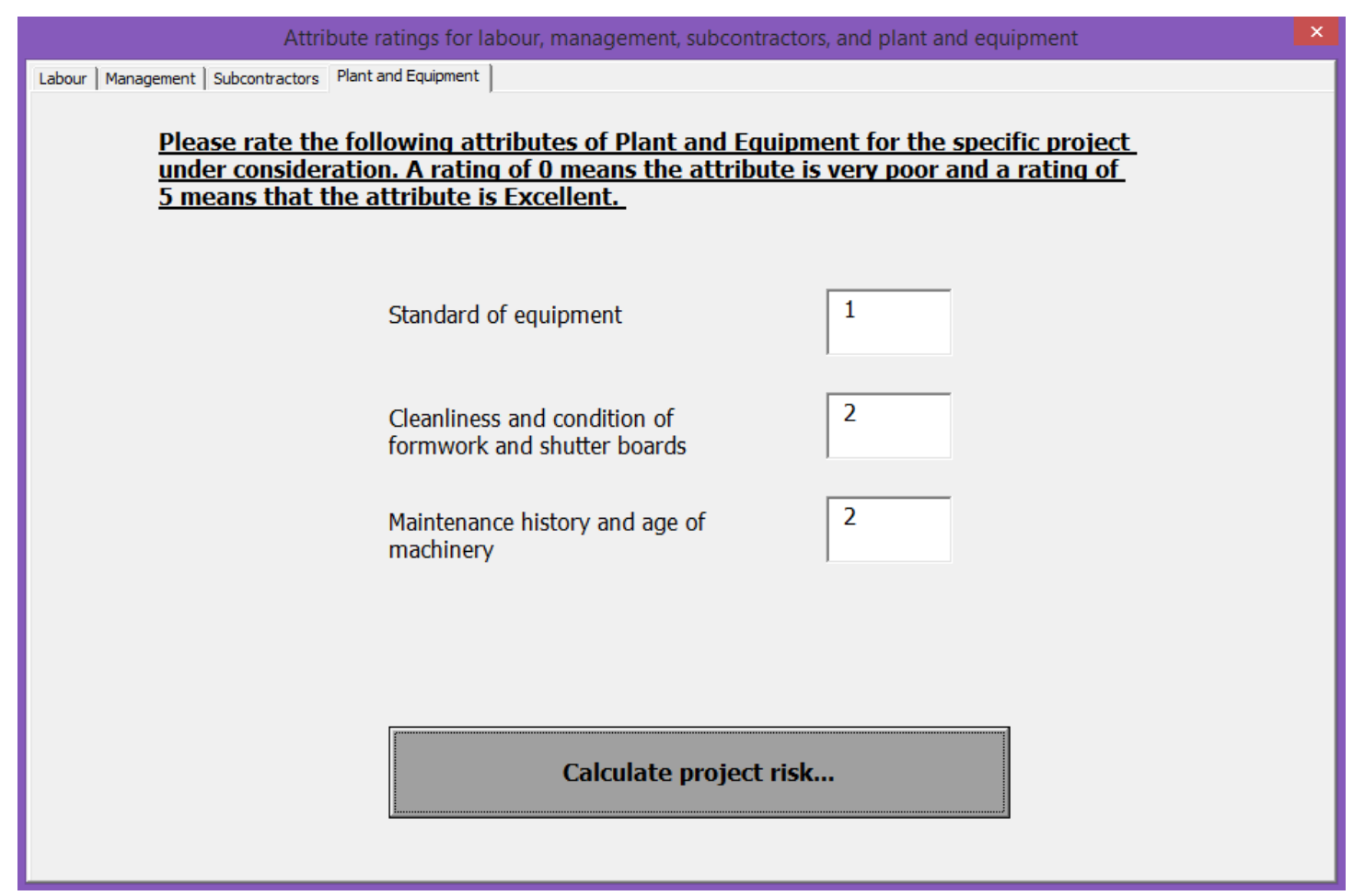

Figure 8.5: Plant and equipment attribute ratings for scenario 1

From the user input presented in Figures 8.2 to 8.5 , the weighted average of each category is calculated. The logic of this process is shown in Step 2 of the model as presented in Chapter 7. Recall that these values are used to determine the likelihood value of each category when calculating the risk magnitude. The impact values are already stored in the model as obtained through Questions 9-12 in the questionnaire survey. The model calculates each of the risk magnitudes, as presented in Step 3 of the model logic in Chapter 7.

The risk magnitudes and corresponding risk classes are presented in Figure 8.6. The user decides which risks require mitigation. For the simulation, the author deliberately chooses to mitigate the risks that are classified as "Medium" or "High." From Figure 8.6 it is shown that most of the risks were classified as "High" because of the poor attribute ratings that were assigned by the author. See Step 3 of Chapter 7 to recall how the risks are classified according to their magnitudes. 


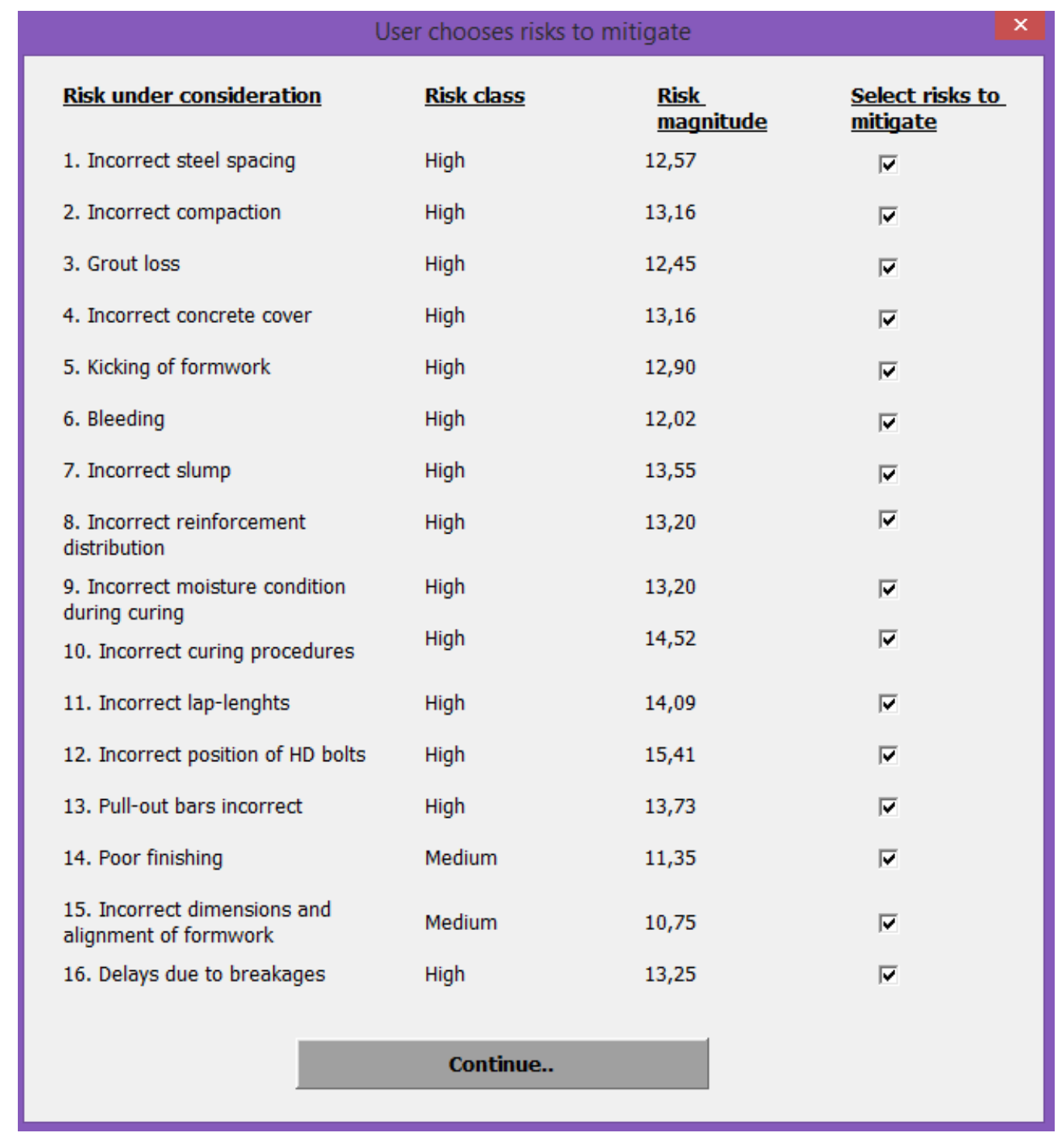

Figure 8.6: Risk magnitudes identified and user chooses which risks to mitigate

After choosing the risks that require mitigation (Step 4 of Chapter 7), the user enters the total value of concrete works on the project as well as the expected rework percentage as caused by the risks that require mitigation.

For the simulation the author chooses an expected total rework value of $3 \%$ on the total value of concrete works. Recall from the questionnaire survey that the expected rework ranges between $4-6 \%$. When applying the model on a real-life project, the user should estimate the expected rework percentage as the rework expected from the risks that require mitigation.

As shown in Figure 8.7, the user assigns a percentage of the expected rework costs to each of the risks. In a real-life project the user would provide these percentages based on previous experiences or company data. For the simulation the rework cost allocation is evenly distributed between all the risks that require mitigation. In Figure 8.7 the cost allocation for each risk is shown as $6.25 \%$ and the sum of all the allocations add up to 
$100 \%$. The $100 \%$ represents the full portion of the expected rework.

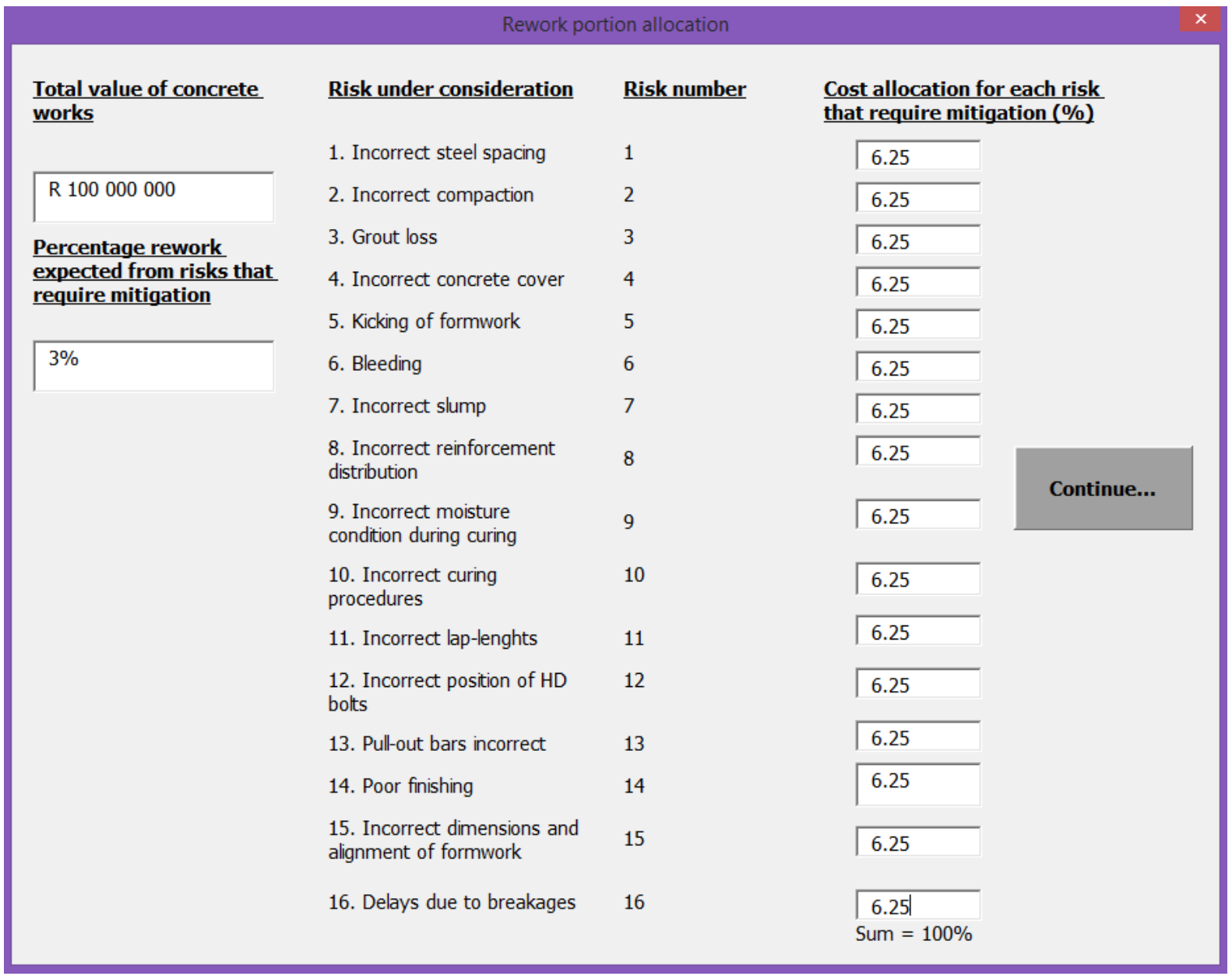

Figure 8.7: Cost allocation of rework expected from each risk that requires mitigation

The model chooses the technological systems (presented in Chapter 4) used to mitigate each risk and calculates the potential rework saving when using the technological systems to mitigate the risks. The rework saving is determined for each of the risks. There is a number of risks that can be mitigated by each technological system. The rework saved by mitigating risks through use of a system is summed for each system. The total rework saved from using each system individually is presented in Figure 8.8. The net profit resulting from the use of each system is determined by subtracting the cost of the system from total rework saved by the system. Figure 8.8 presents the values in the cost calculations. The logic of this step is presented as Step 5 in Chapter 7.

The model considers two cases when calculating the net savings from using the laser scanner and the image dimensioning software (IDS). Some of the risks can be mitigated by using either of these technologies. In case 1 the model calculates the savings from using only the laser scanner for risks that can be mitigated by both the laser scanner and the IDS. In case 2 the model calculates the net savings from using the IDS to mitigate the risks that can be mitigated by both the laser scanner and the IDS. The model then chooses the case where the net savings is a maximum. Figure 8.8 presents the technology 
systems available, rework reduction from using the technology, cost of each system, net savings from using each system and the total expected savings from all the systems.

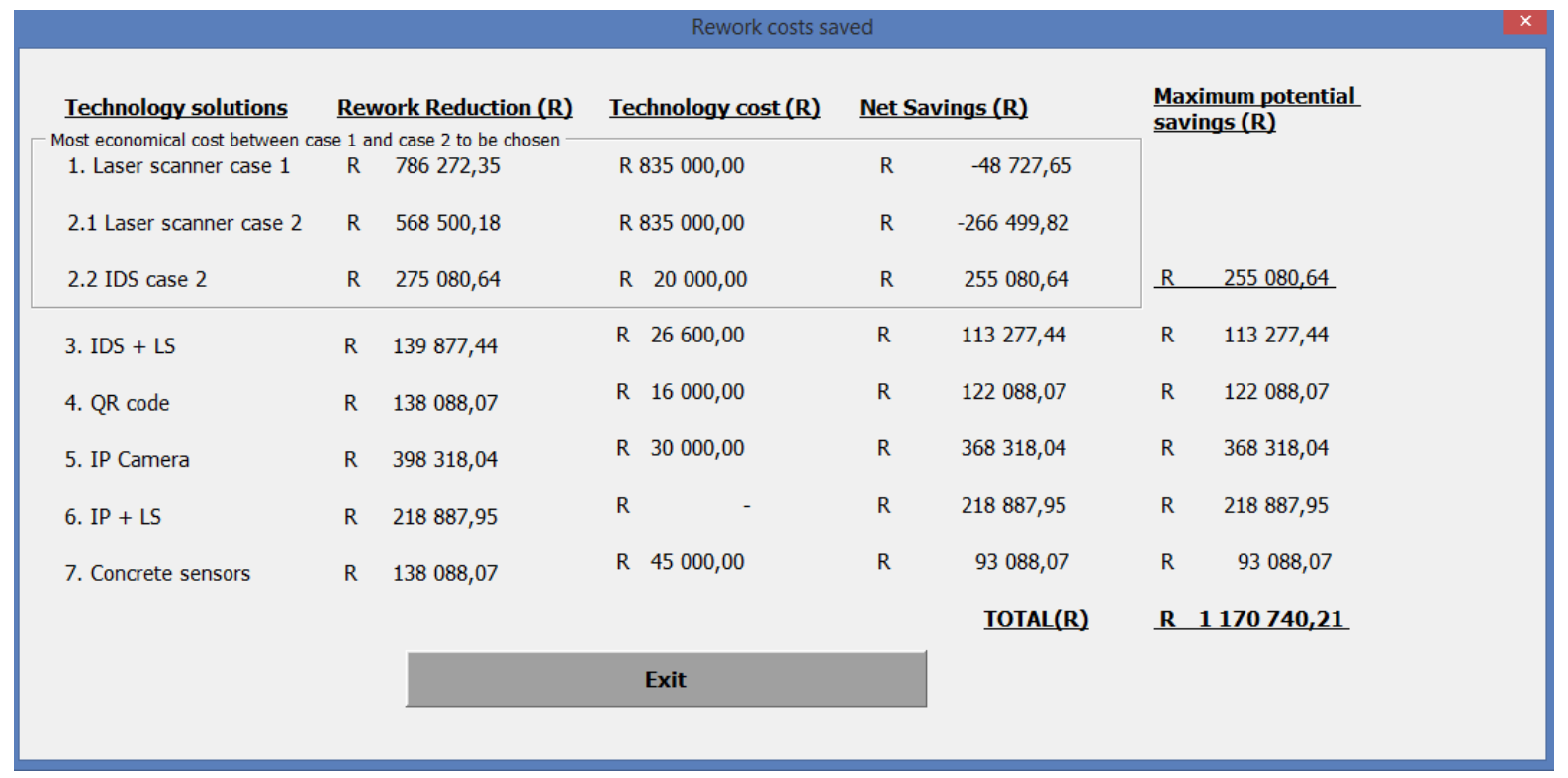

Figure 8.8: Rework savings expected from each technological system for scenario 1

In the example for scenario 1, the total expected net rework saving is R 1170740.21 . This is $39 \%$ of the total rework expected to occur (=1 $170740.21 / 3000000)$. Table 8.1 shows the risks that are mitigated by each technological system and their respective rework reduction. The risks that can be mitigated by the laser scanner are not included since the use of a laser scanner will result in a net loss instead of a net saving.

Table 8.1: Technology used to mitigate each risk and rework saved from mitigating each risk

\begin{tabular}{|c|c|c|c|c|c|}
\hline Technology system & $\begin{array}{l}\text { Acronym in Figure } \\
8.8\end{array}$ & $\begin{array}{l}\text { Risk nr (Figure } \\
8.6\end{array}$ & $\begin{array}{l}\text { Rework reduced } \\
(\mathrm{R})\end{array}$ & $\begin{array}{l}\text { Technology cost } \\
\text { (R) }\end{array}$ & Net savings ( $R$ ) \\
\hline $\begin{array}{l}\text { Image dimensioning } \\
\text { software }\end{array}$ & IDS & 1 & R 129681,72 & 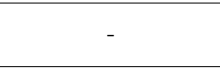 & - \\
\hline $\begin{array}{l}\text { Image dimensioning } \\
\text { software }\end{array}$ & IDS & 11 & R 145398,92 & R 45000,00 & R 255080,64 \\
\hline $\begin{array}{l}\text { Image dimensioning- } \\
\text { and logging software }\end{array}$ & IDS + LS & 7 & R 139877,44 & R 26600,00 & 113277,44 \\
\hline Quick response code & QR code & 8 & R 138088,07 & R 16000,00 & 122088,07 \\
\hline $\begin{array}{l}\text { Internet protocol } \\
\text { camera }\end{array}$ & IP camera & 2 & R 103754,86 & - & - \\
\hline $\begin{array}{l}\text { Internet protocol } \\
\text { camera }\end{array}$ & IP camera & 3 & R 98134,05 & - & - \\
\hline $\begin{array}{l}\text { Internet protocol } \\
\text { camera }\end{array}$ & IP camera & 5 & R 101669,33 & - & - \\
\hline $\begin{array}{l}\text { Internet protocol } \\
\text { camera }\end{array}$ & IP camera & 6 & R 94759,81 & R 30000,00 & R 368318,04 \\
\hline $\begin{array}{l}\text { Internet protocol } \\
\text { camera and logging } \\
\text { software }\end{array}$ & $\mathrm{IP}+\mathrm{LS}$ & 10 & R 114442,86 & - & - \\
\hline $\begin{array}{l}\text { Internet protocol } \\
\text { camera and logging } \\
\text { software }\end{array}$ & $\mathrm{IP}+\mathrm{LS}$ & 16 & R 104445,09 & - & R 218887,95 \\
\hline Concrete sensors & - & 9 & R 138088,07 & R 45000,00 & R 93088,07 \\
\hline Total $(\mathrm{R})$ & - & - & $\sum=\mathrm{R} 1308340,21$ & $\sum=\mathrm{R} 137600,00$ & $\sum=\mathrm{R} 1170740,21$ \\
\hline
\end{tabular}




\subsubsection{Scenario 2: Fairly good performance from management}

For scenario 2 it is deliberately assumed that the contractor decides to send a fairly experienced management team to compensate for poor expectancies from labour, subcontractors, and plant and equipment. The assumptions previously made for all the categories, except for management, remains the same in scenario 2. It is deliberately held the same to demonstrate the change in risk as the management attributes improve.

The following information is available on the site management team:

- Management team known for good coordination on previous projects

- Management meets on weekly time intervals to plan ahead

- Architect included some impractical elements to be constructed

- Management has some experience on quality management and checks to conduct

- There is a fairly well developed quality management system in place

- There are additional supervisors appointed for the project

- One of the supervisors speaks the local language as a second language.

Table 8.2 shows the changed attribute ratings chosen for the more experienced management category in scenario 2 . The other attribute ratings are the same as in scenario 1 . In scenario 1 the attribute ratings were shown in Figures 8.2 to 8.5 to show the application of the software. The same process was followed for scenario 2, but the input is summarised in Table 8.2 instead of showing images of the software input.

Table 8.2: Performance indicators for each category in scenario 2

\begin{tabular}{|c|c|c|c|c|c|c|c|}
\hline Labour & Rating & Management & Rating & Subcontractors & Rating & $\begin{array}{l}\text { Plant and } \\
\text { equipment }\end{array}$ & Rating \\
\hline $\begin{array}{l}\text { Ratio of } \\
\text { casual labour }\end{array}$ & $70 \%$ & Coordination & 4 & Communication & 1 & $\begin{array}{l}\text { Equipment } \\
\text { standard }\end{array}$ & 1 \\
\hline Training & 2 & $\begin{array}{l}\text { Precision } \\
\text { planning }\end{array}$ & 3 & $\begin{array}{l}\text { Construction } \\
\text { methods }\end{array}$ & 2 & $\begin{array}{l}\text { Cleanliness of } \\
\text { formwork, } \\
\text { shutter boards } \\
\text { and } \\
\text { equipment }\end{array}$ & 2 \\
\hline Motivation & 1 & $\begin{array}{l}\text { Practical } \\
\text { design }\end{array}$ & 3 & $\begin{array}{l}\text { Knowledge on } \\
\text { operations }\end{array}$ & 2 & $\begin{array}{l}\text { Machinery } \\
\text { maintenance } \\
\text { and condition }\end{array}$ & 2 \\
\hline Skill level & 1 & $\begin{array}{l}\text { Quality } \\
\text { management } \\
\text { expertise }\end{array}$ & 3 & Experience & 2 & - & - \\
\hline Self-discipline & 2 & $\begin{array}{l}\text { Quality } \\
\text { management } \\
\text { system }\end{array}$ & 3 & - & - & - & - \\
\hline- & - & Supervision & 4 & - & - & - & - \\
\hline- & - & Communication & 3 & - & - & - & - \\
\hline
\end{tabular}

Figure 8.9 shows the magnitude and class of the risks identified. Six of the risks were classified as high, nine risks were classified as medium and one risk was classified as low. 
For the simulation of scenario 2 the author chose not to mitigate the risk classified as low. This is applied for all the subsequent scenarios.

\begin{tabular}{|c|c|c|c|}
\hline \multicolumn{4}{|c|}{ User chooses risks to mitigate } \\
\hline Risk under consideration & Risk class & $\begin{array}{l}\text { Risk } \\
\text { magnitude }\end{array}$ & $\begin{array}{l}\text { Select risks to } \\
\text { mitigate }\end{array}$ \\
\hline 1. Incorrect steel spacing & High & 12,57 & $\sqrt{v}$ \\
\hline 2. Incorrect compaction & High & 13,16 & $\sqrt{v}$ \\
\hline 3. Grout loss & High & 12,34 & v \\
\hline 4. Incorrect concrete cover & High & 13,16 & V \\
\hline 5. Kicking of formwork & High & 12,90 & $\sqrt{v}$ \\
\hline 6. Bleeding & Low & 5,72 & $\Gamma$ \\
\hline 7. Incorrect slump & Medium & 11,48 & $\sqrt{v}$ \\
\hline $\begin{array}{l}\text { 8. Incorrect reinforcement } \\
\text { distribution }\end{array}$ & Medium & 6,28 & v \\
\hline $\begin{array}{l}\text { 9. Incorrect moisture condition } \\
\text { during curing }\end{array}$ & Medium & 6,28 & v \\
\hline 10. Incorrect curing procedures & Medium & 6,91 & $v_{v}$ \\
\hline 11. Incorrect lap-lenghts & Medium & 6,71 & V \\
\hline 12. Incorrect position of HD bolts & Medium & 7,34 & $\sqrt{v}$ \\
\hline 13. Pull-out bars incorrect & Medium & 6,54 & v \\
\hline 14. Poor finishing & Medium & 11,35 & $\sqrt{v}$ \\
\hline $\begin{array}{l}\text { 15. Incorrect dimensions and } \\
\text { alignment of formwork }\end{array}$ & Medium & 10,75 & $\sqrt{v}$ \\
\hline 16. Delays due to breakages & High & 13,25 & v \\
\hline & Continue & & \\
\hline
\end{tabular}

Figure 8.9: Risk magnitudes and classification for scenario 2

In the next step the author enters the concrete works value and estimates the expected rework due to the risks that require mitigation. Recall that in scenario 1 the author used an expected rework percentage of $3 \%$. In a real-life project the user would estimate this value based on the previous experience or company data and the risks that require mitigation.

To include the effect of the reduction in risks that require mitigation, the expected rework percentage is reduced accordingly. The total expected rework percentage was adjusted in line with the reduction in total risk magnitude from scenario 1 to scenario 2. The total sum of risk magnitudes for scenario 1 was 209 and for scenario 2 it was 151 . The risk score includes only the risks that require mitigation. That is a $28 \%$ reduction in the total risk magnitude. Therefore, the expected rework of $3 \%$ in scenario 1 was reduced to $2.17 \%(3 \times 0.72)$ for scenario 2 . This reduction is only determined for the purpose of the simulation.

In the next step the author allocated the expected rework cost evenly between the risks that require mitigation. Recall from scenario 1 that this is divided evenly for demonstrative purposes and in real-life examples the allocation would be based on previous experience or company data. Since scenario 1 presented the detailed steps for the application of the model, scenarios 2 to 5 only show the summary information obtained 
from the simulation. Table 8.3 shows the potential reduction in rework for each of the technological systems.

Table 8.3: Net rework reduction by each technological system for scenario 2

\begin{tabular}{lc}
\hline $\begin{array}{l}\text { Technological } \\
\text { solution }\end{array}$ & $\begin{array}{l}\text { Net rework } \\
\text { savings (R) }\end{array}$ \\
\hline $\begin{array}{l}\text { Image dimensioning } \\
\text { software }\end{array}$ & 179403.20 \\
\hline $\begin{array}{l}\text { Image dimensioning- } \\
\text { and logging software }\end{array}$ & 92214.56 \\
\hline Quick response code & 49901.73 \\
\hline IP camera & 273433.57 \\
\hline $\begin{array}{l}\text { IP camera and } \\
\text { logging software }\end{array}$ & 159328.61 \\
\hline Concrete sensors & 20901.73 \\
\hline Total net savings & 775183.40 \\
\hline \hline
\end{tabular}

The increase in management performance reduced some of the risks and the expected rework to occur. The net potential rework savings, after deducting technology expenses, were determined as R 775 183.40.

\subsubsection{Scenario 3: Fairly good performance from labour and management}

In scenario 3 the author demonstrates the effect of an increase in the performance of labour attributes. The management performance is the same as in scenario 2 since the author aims to demonstrate the reduction in risk when both labour and management are expected to be good. The following information describes the performance expectancy from labour:

- Reduce the casual labour to $50 \%$

- Provide better training to labourers for the project

- Motivate labourers to take pride in their work and community

- Employ labourers available with a higher skill level

- Conduct interviews to identify labourers with good self-discipline.

Table 8.4 shows the attribute ratings for scenario 3. 
Table 8.4: Performance indicators for each category in scenario 3

\begin{tabular}{|c|c|c|c|c|c|c|c|}
\hline Labour & Rating & Management & Rating & Subcontractors & Rating & $\begin{array}{l}\text { Plant and } \\
\text { equipment }\end{array}$ & Rating \\
\hline $\begin{array}{l}\text { Ratio of } \\
\text { casual labour }\end{array}$ & $50 \%$ & Coordination & 4 & Communication & 1 & $\begin{array}{l}\text { Equipment } \\
\text { standard }\end{array}$ & 1 \\
\hline Training & 4 & $\begin{array}{l}\text { Precision } \\
\text { planning }\end{array}$ & 3 & $\begin{array}{l}\text { Construction } \\
\text { methods }\end{array}$ & 2 & $\begin{array}{l}\text { Cleanliness of } \\
\text { formwork, } \\
\text { shutter boards } \\
\text { and } \\
\text { equipment }\end{array}$ & 2 \\
\hline Motivation & 4 & $\begin{array}{l}\text { Practical } \\
\text { design }\end{array}$ & 3 & $\begin{array}{l}\text { Knowledge on } \\
\text { operations }\end{array}$ & 2 & $\begin{array}{l}\text { Machinery } \\
\text { maintenance } \\
\text { and condition }\end{array}$ & 2 \\
\hline Skill level & 3 & $\begin{array}{l}\text { Quality } \\
\text { management } \\
\text { expertise }\end{array}$ & 3 & Experience & 2 & - & - \\
\hline Self-discipline & 4 & $\begin{array}{l}\text { Quality } \\
\text { management } \\
\text { system }\end{array}$ & 3 & - & - & - & - \\
\hline - & - & Supervision & 4 & - & - & - & - \\
\hline - & - & Communication & 3 & - & - & - & - \\
\hline
\end{tabular}

Figure 8.10 shows the risk magnitudes determined from the input in Table 8.4. Only three risks were now identified as high-, nine were identified as medium- and four were identified as low risk. Recall from the previous scenarios that low risks are not considered for mitigation. The total risk score is reduced to 111.74 after improving labour attributes. This represents a $26 \%$ reduction in total risk. Furthermore, was the total expected rework cost reduced accordingly to $1.60 \%(111.74 / 151 \times 2.17 \%)$. Thus, the total expected rework cost on the project is now $\mathrm{R} 1600000.00$. 


\begin{tabular}{|c|c|c|c|}
\hline \multicolumn{4}{|c|}{ User chooses risks to mitigate } \\
\hline Risk under consideration & Risk class & $\begin{array}{l}\text { Risk } \\
\text { magnitude }\end{array}$ & $\begin{array}{l}\text { Select risks to } \\
\text { mitigate }\end{array}$ \\
\hline 1. Incorrect steel spacing & High & 12,55 & $\nabla$ \\
\hline 2. Incorrect compaction & Low & 5,21 & $\Gamma$ \\
\hline 3. Grout loss & Low & 5,93 & г \\
\hline 4. Incorrect concrete cover & High & 12,29 & $\nabla$ \\
\hline 5. Kicking of formwork & Low & 5,10 & $\Gamma$ \\
\hline 6. Bleeding & Low & 5,72 & г \\
\hline 7. Incorrect slump & Medium & 11,48 & $\nabla$ \\
\hline $\begin{array}{l}\text { 8. Incorrect reinforcement } \\
\text { distribution }\end{array}$ & Medium & 6,28 & $\sqrt{v}$ \\
\hline $\begin{array}{l}\text { 9. Incorrect moisture condition } \\
\text { during curing }\end{array}$ & Medium & 6,28 & $\nabla$ \\
\hline 10. Incorrect curing procedures & Medium & 6,91 & $\bar{v}$ \\
\hline 11. Incorrect lap-lenghts & Medium & 6,71 & $\nabla$ \\
\hline 12. Incorrect position of HD bolts & Medium & 7,34 & $\nabla$ \\
\hline 13. Pull-out bars incorrect & Medium & 6,54 & $\nabla$ \\
\hline 14. Poor finishing & Medium & 11,35 & $\nabla$ \\
\hline $\begin{array}{l}\text { 15. Incorrect dimensions and } \\
\text { alignment of formwork }\end{array}$ & Medium & 10,75 & $\nabla$ \\
\hline 16. Delays due to breakages & High & 13,25 & $\nabla$ \\
\hline & Continue & & \\
\hline
\end{tabular}

Figure 8.10: Risk magnitudes and classification for scenario 3

The net savings were determined after considering the risk mitigation and allocating rework costs to the each of the remaining risks. The net savings on rework are shown in Table 8.5. Again it was found that a net loss would result through use of the laser scanner. Therefore, it is not included in Table 8.5. The total rework reduction results in $\mathrm{R} 442$ 870.05. This is much less than previously calculated because of the reduction in project risk. Lower risk leads to less rework expected; therefore, less rework savings (R) are expected through technology mitigation. 
Table 8.5: Net rework reduction by each technological system for scenario 3

\begin{tabular}{|c|c|}
\hline $\begin{array}{l}\text { Technological } \\
\text { solution }\end{array}$ & $\begin{array}{l}\text { Net rework } \\
\text { savings (R) }\end{array}$ \\
\hline $\begin{array}{l}\text { Image dimensioning } \\
\text { software }\end{array}$ & 178554.51 \\
\hline $\begin{array}{l}\text { Image dimensioning- } \\
\text { and logging software }\end{array}$ & 91799.79 \\
\hline Quick response code & 49671.67 \\
\hline $\begin{array}{l}\text { IP camera and } \\
\text { logging software }\end{array}$ & 102172.41 \\
\hline Concrete sensors & 20671.67 \\
\hline Total net savings & 442870.05 \\
\hline
\end{tabular}

\subsubsection{Scenario 4: Fairly good performance from labour, man- agement and subcontractors}

In scenario 4 it is assumed, for demonstrative purposes, that good performance can be expected from all the categories except for plant and equipment. The attribute ratings chosen in scenario 3 for labour, management, and plant and equipment remain the same. The following assumptions were made regarding the subcontractors:

- The subcontractor is known for communicating well with all stakeholders

- The subcontractor uses advanced construction methods which yield good results

- The subcontractor has got prior knowledge on the type of construction

- The subcontractor has at least 10 years experience.

The attribute ratings for scenario 4 is shown in Table 8.6 . 
Table 8.6: Attribute ratings for each category in scenario 4

\begin{tabular}{|c|c|c|c|c|c|c|c|}
\hline Labour & Rating & Management & Rating & Subcontractors & Rating & $\begin{array}{l}\text { Plant and } \\
\text { equipment }\end{array}$ & Rating \\
\hline $\begin{array}{l}\text { Ratio of } \\
\text { casual labour }\end{array}$ & $50 \%$ & Coordination & 4 & Communication & 4 & $\begin{array}{l}\text { Equipment } \\
\text { standard }\end{array}$ & 1 \\
\hline Training & 4 & $\begin{array}{l}\text { Precision } \\
\text { planning }\end{array}$ & 3 & $\begin{array}{l}\text { Construction } \\
\text { methods }\end{array}$ & 3 & $\begin{array}{l}\text { Cleanliness of } \\
\text { formwork, } \\
\text { shutter boards } \\
\text { and } \\
\text { equipment }\end{array}$ & 2 \\
\hline Motivation & 4 & $\begin{array}{l}\text { Practical } \\
\text { design }\end{array}$ & 3 & $\begin{array}{l}\text { Knowledge on } \\
\text { operations }\end{array}$ & 4 & $\begin{array}{l}\text { Machinery } \\
\text { maintenance } \\
\text { and condition }\end{array}$ & 2 \\
\hline Skill level & 3 & $\begin{array}{l}\text { Quality } \\
\text { management } \\
\text { expertise }\end{array}$ & 3 & Experience & 5 & - & - \\
\hline Self-discipline & 4 & $\begin{array}{l}\text { Quality } \\
\text { management } \\
\text { system }\end{array}$ & 3 & - & - & - & - \\
\hline - & - & Supervision & 4 & - & - & - & - \\
\hline - & - & Communication & 3 & - & - & - & - \\
\hline
\end{tabular}

The model shows that there was now only one risk identified as high-, nine identified as medium- and six identified as low risk. The total risk magnitude for risks that require mitigation is now reduced to 81.86 . This represents a reduction of $27 \%$ in risk compared to scenario 3. The total rework expectancy is accordingly reduced to $1.17 \%$ $\left(81.86 / 111.74^{*} 1.60\right)$. There still is a number of medium classified risks that increase the total risk magnitude. The reason therefore is that the attributes were not given very high ratings since the performance expectancy is good, but not excellent. Figure 8.11 shows the risk identification for scenario 4 . 


\begin{tabular}{|c|c|c|c|}
\hline \multicolumn{4}{|c|}{ User chooses risks to mitigate } \\
\hline Risk under consideration & Risk class & $\underline{\text { Risk }}$ & $\begin{array}{l}\text { Select risks to } \\
\text { mitigate }\end{array}$ \\
\hline 1. Incorrect steel spacing & LOW & 4,97 & $\Gamma$ \\
\hline 2. Incorrect compaction & LOW & 5,21 & $\Gamma$ \\
\hline 3. Grout loss & LOW & 5,93 & $\Gamma$ \\
\hline 4. Incorrect concrete cover & LOW & 5,21 & $\Gamma$ \\
\hline 5. Kicking of formwork & LOW & 5,10 & $\Gamma$ \\
\hline 6. Bleeding & LOW & 5,72 & $\Gamma$ \\
\hline 7. Incorrect slump & Medium & 6,45 & $\sqrt{\square}$ \\
\hline $\begin{array}{l}\text { 8. Incorrect reinforcement } \\
\text { distribution }\end{array}$ & Medium & 6,28 & $\sqrt{\nabla}$ \\
\hline $\begin{array}{l}\text { 9. Incorrect moisture condition } \\
\text { during curing }\end{array}$ & Medium & 6,28 & $\sqrt{\nabla}$ \\
\hline 10. Incorrect curing procedures & Medium & 6,91 & $\sqrt{\nabla}$ \\
\hline 11. Incorrect lap-lenghts & Medium & 6,71 & $\sqrt{v}$ \\
\hline 12. Incorrect position of HD bolts & Medium & 7,34 & $\sqrt{\nabla}$ \\
\hline 13. Pull-out bars incorrect & Medium & 6,54 & $\sqrt{\nabla}$ \\
\hline 14. Poor finishing & Medium & 11,35 & $\sqrt{\nabla}$ \\
\hline $\begin{array}{l}\text { 15. Incorrect dimensions and } \\
\text { alignment of formwork }\end{array}$ & Medium & 10,75 & $\sqrt{\nabla}$ \\
\hline \multirow[t]{2}{*}{ 16. Delays due to breakages } & High & 13,25 & $\sqrt{\nabla}$ \\
\hline & Continue & & \\
\hline
\end{tabular}

Figure 8.11: Risk magnitudes and classification for scenario 4

The net savings amount in scenario 4 is $\mathrm{R} 260$ 790.29. It is still profitable to make the technological investment to reduce rework. Table 8.7 shows the rework reduction from each of the technological solutions. The laser scanner is still not profitable to use.

Table 8.7: Net rework reduction by each technological system for scenario 4

\begin{tabular}{|c|c|}
\hline $\begin{array}{l}\text { Technological } \\
\text { solution }\end{array}$ & $\begin{array}{l}\text { Net rework } \\
\text { savings (R) }\end{array}$ \\
\hline $\begin{array}{l}\text { Image dimensioning } \\
\text { software }\end{array}$ & 49019.17 \\
\hline $\begin{array}{l}\text { Image dimensioning- } \\
\text { and logging software }\end{array}$ & 39798.19 \\
\hline Quick response code & 49548.80 \\
\hline $\begin{array}{l}\text { IP camera and } \\
\text { logging software }\end{array}$ & 101875.34 \\
\hline Concrete sensors & 20548.80 \\
\hline Total net savings & 260790.29 \\
\hline
\end{tabular}




\subsubsection{Scenario 5: Good performance from all categories and very good management performance}

In scenario 5 it is expected that all the categories show good performance. The plant and equipment and management attributes are improved in this scenario. The labour and subcontractor ratings remain the same as in scenario 4 . The attribute ratings are shown in Table 8.8.

Table 8.8: Attribute ratings for each category in scenario 5

\begin{tabular}{|c|c|c|c|c|c|c|c|}
\hline Labour & Rating & Management & Rating & Subcontractors & Rating & $\begin{array}{l}\text { Plant and } \\
\text { equipment }\end{array}$ & Rating \\
\hline $\begin{array}{l}\text { Ratio of } \\
\text { casual labour }\end{array}$ & $50 \%$ & Coordination & 4 & Communication & 4 & $\begin{array}{l}\text { Equipment } \\
\text { standard }\end{array}$ & 3 \\
\hline Training & 4 & $\begin{array}{l}\text { Precision } \\
\text { planning }\end{array}$ & 4 & $\begin{array}{l}\text { Construction } \\
\text { methods }\end{array}$ & 3 & $\begin{array}{l}\text { Cleanliness of } \\
\text { formwork, } \\
\text { shutter boards } \\
\text { and } \\
\text { equipment }\end{array}$ & 3 \\
\hline Motivation & 4 & $\begin{array}{l}\text { Practical } \\
\text { design }\end{array}$ & 3 & $\begin{array}{l}\text { Knowledge on } \\
\text { operations }\end{array}$ & 4 & $\begin{array}{l}\text { Machinery } \\
\text { maintenance } \\
\text { and condition }\end{array}$ & 4 \\
\hline Skill level & 3 & $\begin{array}{l}\text { Quality } \\
\text { management } \\
\text { expertise }\end{array}$ & 5 & Experience & 5 & - & - \\
\hline Self-discipline & 4 & $\begin{array}{l}\text { Quality } \\
\text { management } \\
\text { system }\end{array}$ & 4 & - & - & - & - \\
\hline - & - & Supervision & 5 & - & - & - & - \\
\hline - & - & Communication & 4 & - & - & - & - \\
\hline
\end{tabular}

The risks are shown in Figure 8.12, It is seen that only one risk requires mitigation. All the other risks are accepted since it was classified as being low. The total risk magnitude, for the risk that requires mitigation, was reduced to 6.67 . The total risk magnitude is used to reduce the total expected rework cost in the project. In real-life the user would make own estimations. The expected rework value is reduced accordingly to $0.096 \%$. The rework value expected from the risk is R96 000.00. 


\begin{tabular}{|c|c|c|c|}
\hline \multicolumn{4}{|c|}{ User chooses risks to mitigate } \\
\hline Risk under consideration & Risk class & $\frac{\text { Risk }}{\text { magnitude }}$ & $\begin{array}{l}\text { Select risks to } \\
\text { mitigate }\end{array}$ \\
\hline 1. Incorrect steel spacing & Low & 4,97 & $\Gamma$ \\
\hline 2. Incorrect compaction & LOW & 5,21 & $\Gamma$ \\
\hline 3. Grout loss & Low & 4,88 & $\Gamma$ \\
\hline 4. Incorrect concrete cover & LOW & 5,21 & $\Gamma$ \\
\hline 5. Kicking of formwork & Low & 5,10 & $\Gamma$ \\
\hline 6. Bleeding & Low & 2,84 & $\Gamma$ \\
\hline 7. Incorrect slump & Low & 3,47 & $\Gamma$ \\
\hline $\begin{array}{l}\text { 8. Incorrect reinforcement } \\
\text { distribution }\end{array}$ & LOW & 3,12 & $\Gamma$ \\
\hline $\begin{array}{l}\text { 9. Incorrect moisture condition } \\
\text { during curing }\end{array}$ & Low & 3,12 & $\Gamma$ \\
\hline 10. Incorrect curing procedures & Low & 3,43 & $\Gamma$ \\
\hline 11. Incorrect lap-lenghts & Low & 3,33 & $\Gamma$ \\
\hline 12. Incorrect position of $\mathrm{HD}$ bolts & Low & 3,64 & $\Gamma$ \\
\hline 13. Pull-out bars incorrect & Low & 3,25 & $\Gamma$ \\
\hline 14. Poor finishing & Low & 5,71 & $\Gamma$ \\
\hline $\begin{array}{l}\text { 15. Incorrect dimensions and } \\
\text { alignment of formwork }\end{array}$ & Low & 5,41 & $\Gamma$ \\
\hline 16. Delays due to breakages & Medium & 6,67 & V \\
\hline & & & \\
\hline
\end{tabular}

Figure 8.12: Risk magnitudes and classification for scenario 5

The model shows that IP camera with the logging software is the only system available to mitigate the risk. The total cost of the system is R 56600.00 , but only R 52800.00 worth of rework could be prevented by the system. Since the rework reduction is less than the cost of the system, no investment should be made. If the investment was made, a net loss of R 3800.00 is expected.

\subsubsection{Summary of various scenarios}

Five scenarios were simulated through use of the model. The first scenario assumes poor attributes for all the categories under consideration. The attributes of all the categories subsequently improve up to scenario 5 . It was shown that the net savings from investing in technology to reduce rework, reduces as the attributes improve. The improved attributes result in reduced risk on the project. A reduction in risk reduces the risks that require mitigation and subsequently reduces the possible savings from mitigating risks.

Figure 8.13 shows the reduction in net savings as the risk is reduced on a project. From Figure 8.13 it can be assumed that the technology mitigation measures are not useful for projects with low risk or projects with a low expected rework percentage. However, in the subsequent Section, the influence of project value and the sensitivity of rework percentage is analysed.

The results in this Section do not provide expected rework reduction values for real-life projects. However, it can be concluded that the feasibility of the technology investment 
increases together with project risk and expected rework. The simulations were conducted, while underwritten by various assumptions, to illustrate the influence of the attribute ratings on the risk magnitudes. Furthermore, it was also used to illustrate the influence of risk on rework values. For a real-life project the user would not evenly distribute the cost of rework between each risk since everyone has different previous experiences and company data.

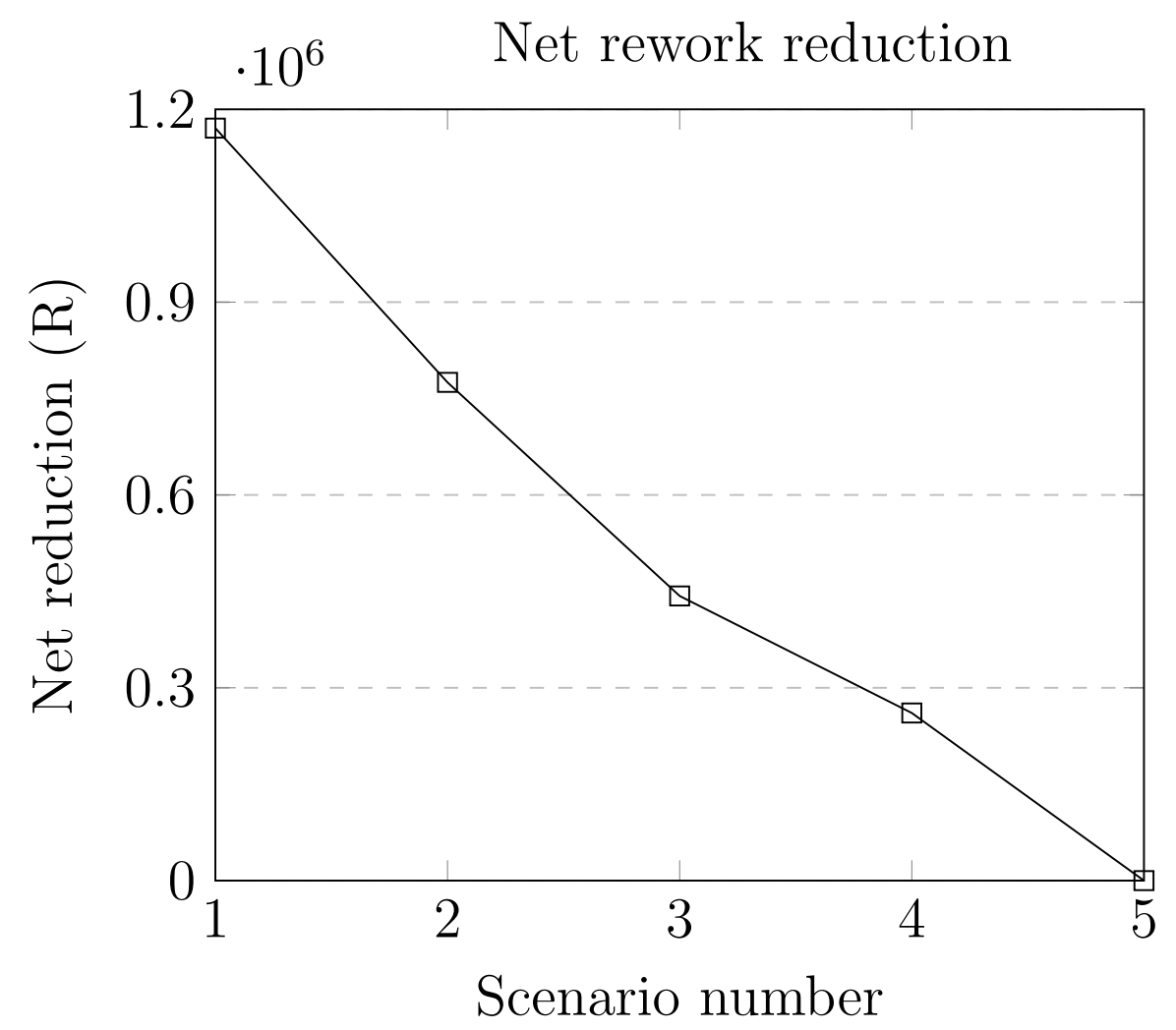

Figure 8.13: Reduction in rework for scenarios 1 to 5

\subsection{Sensitivity analysis}

In this Section, sensitivity analyses were conducted to determine the sensitivity of the output provided by the decision model. Sensitivity analyses were conducted to determine the sensitivity with regards to the following:

- The minimum project value required for net savings to be $>0$

- The net rework savings as the project concrete works value changes

- The net rework savings as the expected rework percentage changes.

The first objective of the sensitivity analyses is to determine the minimum value of concrete works required for the technology investment to be feasible. To achieve this, the value of concrete works was reduced iteratively until the net savings on the project due to risk mitigation was equal to zero, while keeping rework percentage constant. This was 
repeated for various rework percentages ranging from $0.2 \%$ to $4 \%$.

The second objective of the sensitivity analyses is to determine the variability of the net rework savings as the project value changes. To achieve this the concrete works value was deliberately changed for each analysis while keeping the rework percentage constant. This was repeated for rework percentages ranging from $0.2 \%$ to $4 \%$. From the survey results in Chapter 6 it was found that projects typically have rework on concrete that is between $4-6 \%$.

The third objective of the sensitivity analyses is to determine the variability of the net rework savings as the expected rework percentage changes. To achieve this the expected rework percentage was deliberately changed for each analysis while keeping the value of concrete works constant.

For the sensitivity analyses it is assumed that the technologies are used which would result in profitable savings. Therefore, the results that are presented from the analyses include the technology costs of those technologies that result in net profit. However, the technological costs are not shown separately for the analyses.

Figure 8.14 shows a brief outline of the analyses objectives. 


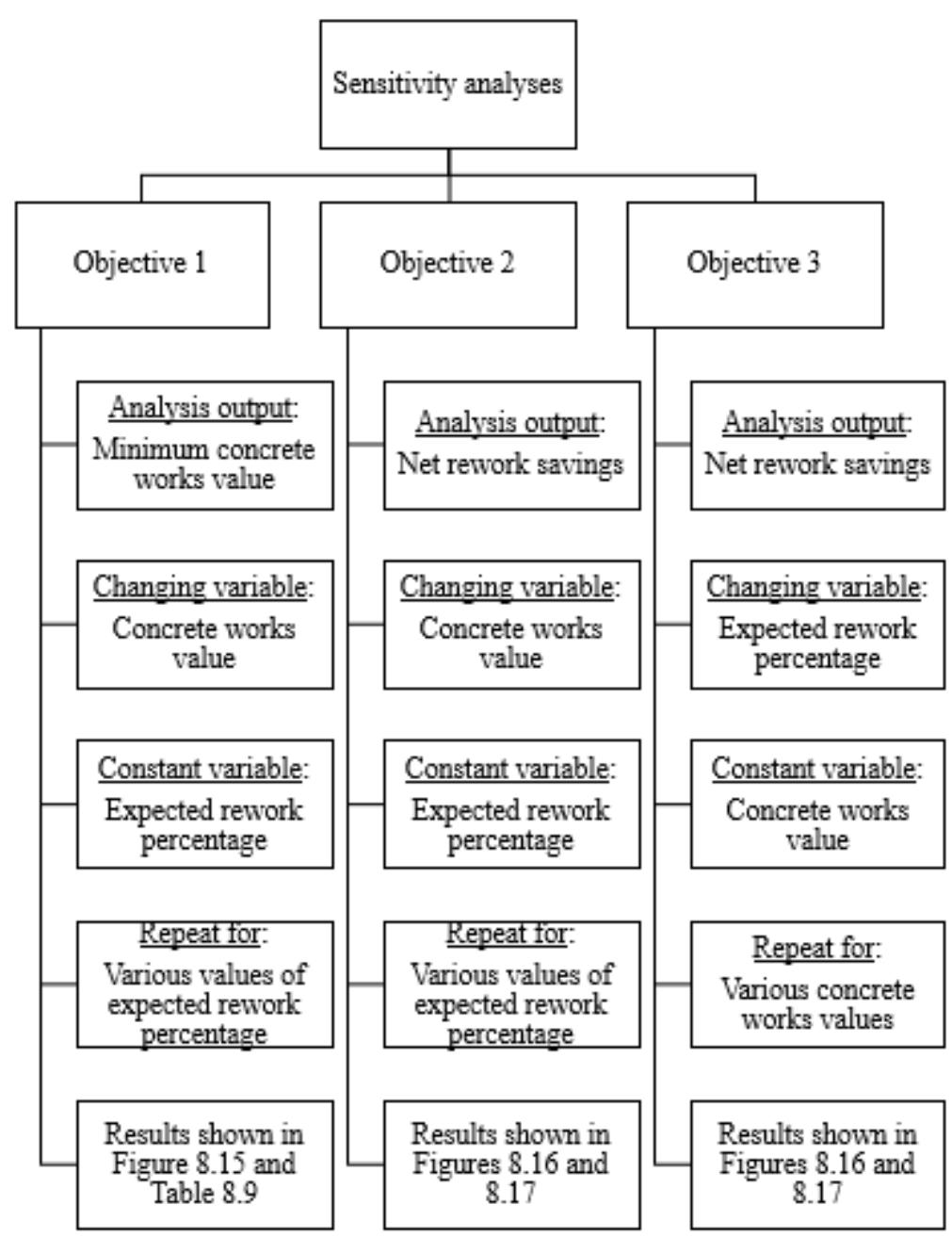

Figure 8.14: Brief outline of sensitivity analyses objectives

When conducting a sensitivity analysis it is required that all the variables in the model be held constant except for those that are deliberately changed to analyse the subsequent response of the model.

The following variables were changed in the model to measure the required sensitivity outcomes.

- Value of concrete works

- Expected rework percentage.

It is assumed that the use of technology for risk mitigation would be the most effective for projects where high risk is expected, since a higher rework value would occur. For demonstrative purposes, high project risk was assumed in the sensitivity analyses. Therefore, the attribute ratings that are required as input into the model (Step 1) were chosen in such a manner that the average risk on the project is classified as high risk.

In the analyses, the total risk magnitude was constantly held at high risk. The sum of the risk magnitudes for a project is classified as high when the total is >192 (16 risks at 
magnitude $>12$ ). This is found by multiplying the high risk requirement value (Risk $>12$ as presented in Table 7.8 of Chapter 7) with the number of risks(16) considered by the model (Total risk magnitude $=12^{*} 16$ ). The attribute inputs (Step 1) were deliberately chosen by the author in such a manner that the total risk magnitude was 193.50.

For the purpose of the analyses the author distributed the expected rework cost proportionately between all the risks. This is held constant for all of the sensitivity analyses. Since there are 16 risks that require mitigation, the proportion of rework cost allocated to each risk is $6.25 \%\left(100 / 16^{*} 100\right)$. In a real-life example the user would allocate the costs disproportionately between the risks according to previous experiences.

\subsubsection{Objective 1: Minimum concrete works value as expected rework percentage changes}

To achieve the first objective a total of 8 analyses were conducted. Table 8.9 shows the minimum feasible project value versus the expected rework value as determined through the analyses. The results in Table 8.9 are limited to the assumptions of the analyses. Therefore, it is specific to the conditions assumed for the analyses and do not represent results for other projects. However, it is useful to illustrate how the the minimum project value required for a feasible investment changes when the expected rework percentage changes. The minimum project values in Table 8.9 represent a break-even point where profit is realisable for various rework percentages. This only pertains to the assumed project conditions.

An example follows to illustrate the interpretation of the analysis results:

A contractor estimates a rework value of $4 \%$ of the concrete works value. According to the model it would be feasible to invest in technology if the concrete works exceed at least $\mathrm{R} 5.5$ million. If the contractor made an initial over estimation error so that the actual rework percentage is only $3 \%$ (instead of 4\%) then the real rework costs would have been $3 \%$ of the concrete works. Then the minimum value of concrete works should exceed at least R 7.3 million for the technology investment to be feasible. If the contractor made an even larger estimation error, where the actual rework value is $1 \%$ of the concrete works (instead of 4\%), the concrete works should exceed R 21 million for the technology investment to be feasible.

From Table 8.9 it is seen that the model is sensitive for rework percentages that are less than $1 \%$ of the concrete works value. The minimum value of concrete works vary between R21 million and R 100 million when considering expected rework values less than $1 \%$. If the contractor estimates a rework percentage of $1 \%$, but the actual rework percentage is $0.5 \%$ then the minimum value of concrete works is increased from $\mathrm{R} 21$ million to $\mathrm{R}$ 44 million. Thus, the concrete works value should exceed R44 million before a profit is realised.

The reason for the high sensitivity when rework percentages are low is caused by the initial cost of the technology. When the expected rework percentage is low then less savings realise from mitigating rework. There is a smaller difference in the technology 
cost and rework saved; therefore, larger concrete work values are required to increase the rework saved.

Table 8.9: Concrete works value required for technological investment to be feasible

\begin{tabular}{cc}
\hline $\begin{array}{c}\text { Rework cost as } \\
\text { percentage of } \\
\text { concrete works value }\end{array}$ & $\begin{array}{c}\text { Minimum concrete } \\
\text { works value before } \\
\text { profit realization } \\
\text { (R-million) }\end{array}$ \\
\hline $4 \%$ & 5.5 \\
\hline $3 \%$ & 7.3 \\
\hline $2 \%$ & 11 \\
\hline $1 \%$ & 21 \\
\hline $0.5 \%$ & 44 \\
\hline $0.4 \%$ & 55 \\
\hline $0.3 \%$ & 73 \\
\hline $0.2 \%$ & 100 \\
\hline
\end{tabular}

Figure 8.15 presents the results in Table 8.9. In Figure 8.15 it is seen that the required value of concrete works increase as the expected rework percentage decreases. A steep gradient is present in the lower expected rework percentage range. It is advised that such a model be used for projects where the expected rework percentage is above rework percentages where the gradient is steep. The area where steep gradients are present is referred to as the sensitive zone and is hatched in Figure 8.15.

Each project will yield a different sensitive zone depending on the model input. For the conditions under which the analyses had been conducted (as presented in Figure8.15), it is advised that the model not be used for rework estimations that are less than $1 \%$ of concrete works value, unless the value of concrete works exceed $\mathrm{R} 100$ million. The $\mathrm{R}$ 100 million value on the $y$-axis correspond with an expected rework cost of $0.20 \%$. Thus, if rework expectancy is only $0.20 \%$ then the concrete works value should exceed $\mathrm{R} 100$ million before net savings are $>0$. The formula in Figure 8.15 represents the minimum value of concrete works (y) required for an expected rework percentage (x) for the assumed conditions. 


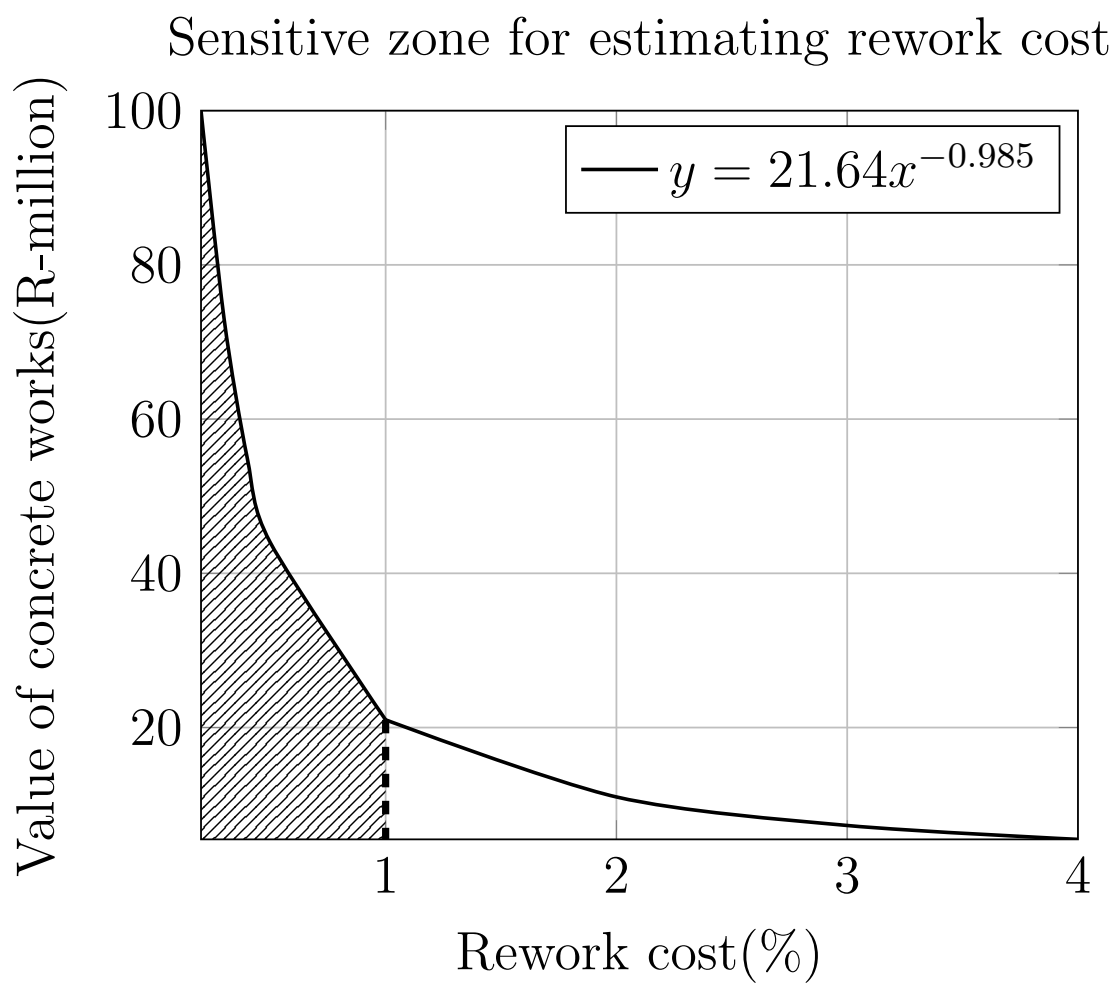

Figure 8.15: Sensitive zone for analyses that were conducted

\subsubsection{Objectives 2 and 3: Variability of net rework savings}

The analyses results for objectives 2 and 3 are combined in Figures 8.16 and 8.18, Figures 8.16 and 8.18 show the net savings (losses) expected, for the assumed project conditions, from using the technology to mitigate risk. The net savings (losses) are shown for a range of project values and expected rework percentages.

To achieve objective 2 , the concrete works value was deliberately changed for each analysis while keeping the rework percentage constant. This was repeated for rework percentages ranging from $0.2 \%$ to $4 \%$.

To achieve the third objective, the expected rework percentage was deliberately changed for each analysis while keeping the value of concrete works constant.

It is shown in Figure 8.16 that net savings do result for most cases where the project value exceeds R 20 million and where the expected rework cost is more than $2 \%$. Figure 8.18 shows the net loss that realises for investing in technology when low rework percentages are expected. The results presented in Figures 8.16 and 8.18 are shown in Table 8.10. It is seen that the technology investment is more applicable to projects where the value of concrete works is large and where the expected rework percentage is high. The legend at the bottom of Figures 8.16 and 8.18 represent the project value used in the analyses. 


\section{Rework savings versus expected rework value}

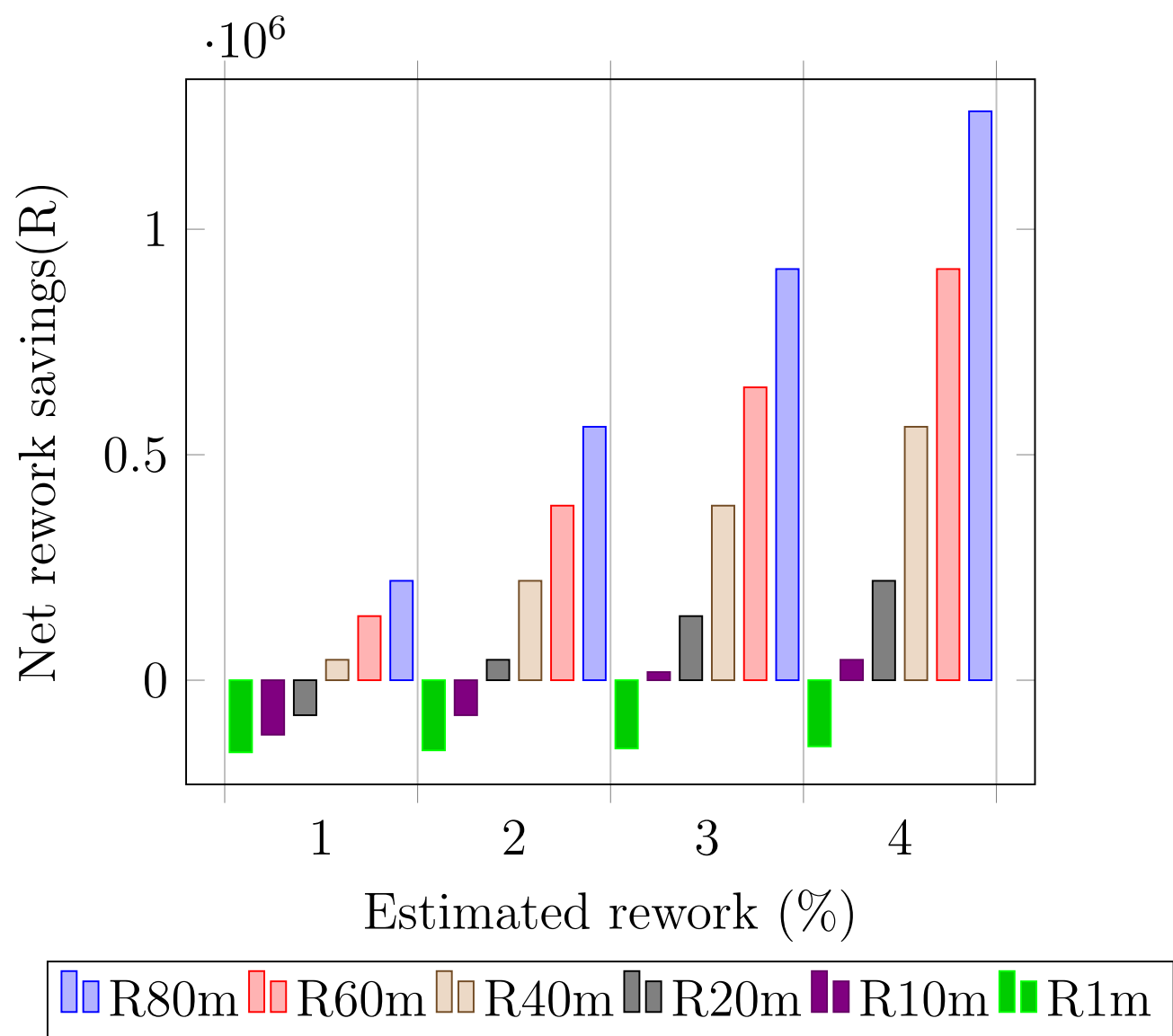

Figure 8.16: Graph showing expected rework reduction versus estimated rework values

Figure 8.17 also shows the data output for the sensitivity analyses presented in Figure 8.16. The net rework savings is shown for various concrete works values as the expected rework percentage changes. The legend in Figure 8.17 represents the rework percentages used in the analyses. 
Rework savings versus concrete works value

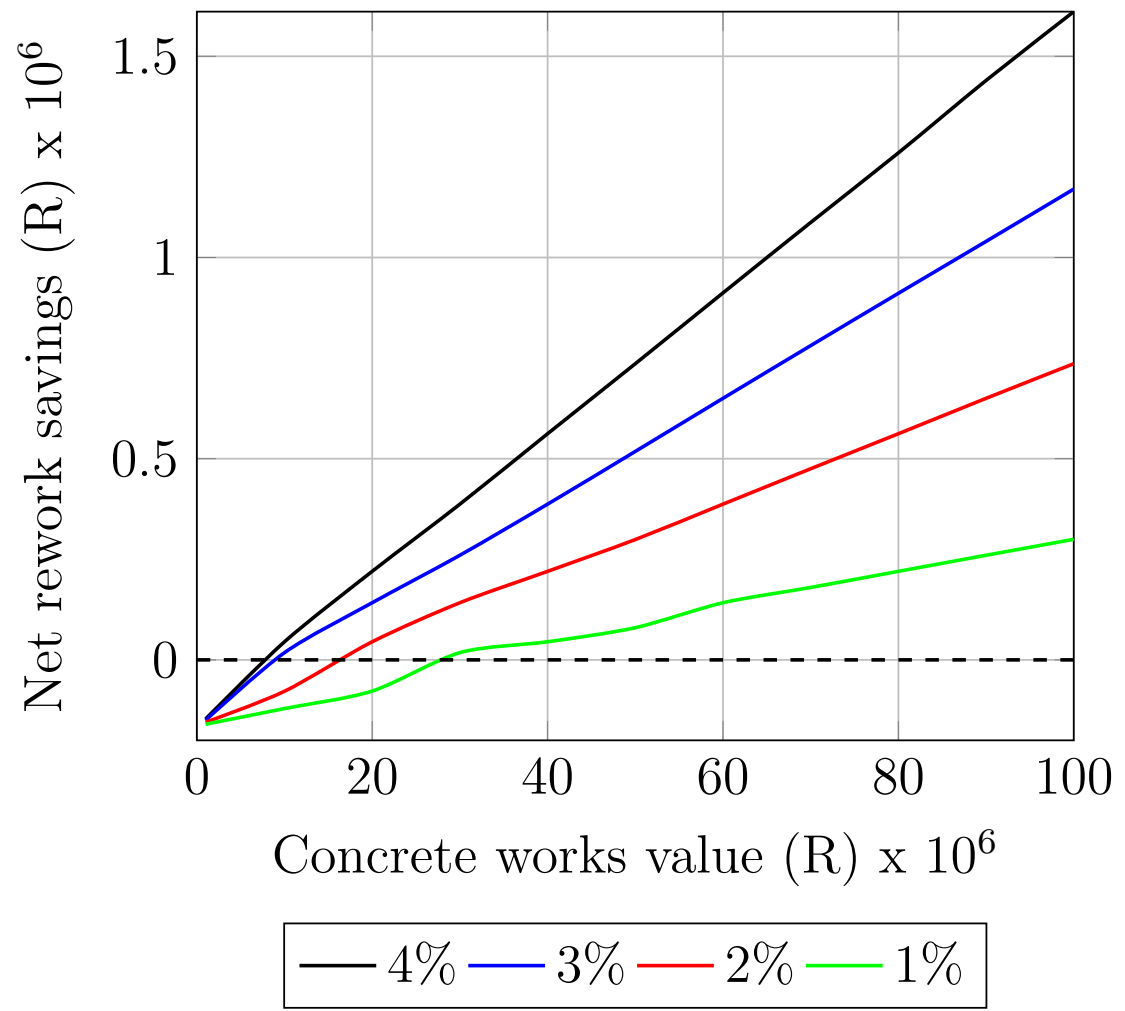

Figure 8.17: Rework savings versus concrete works value 


\section{Rework savings versus expected rework value}

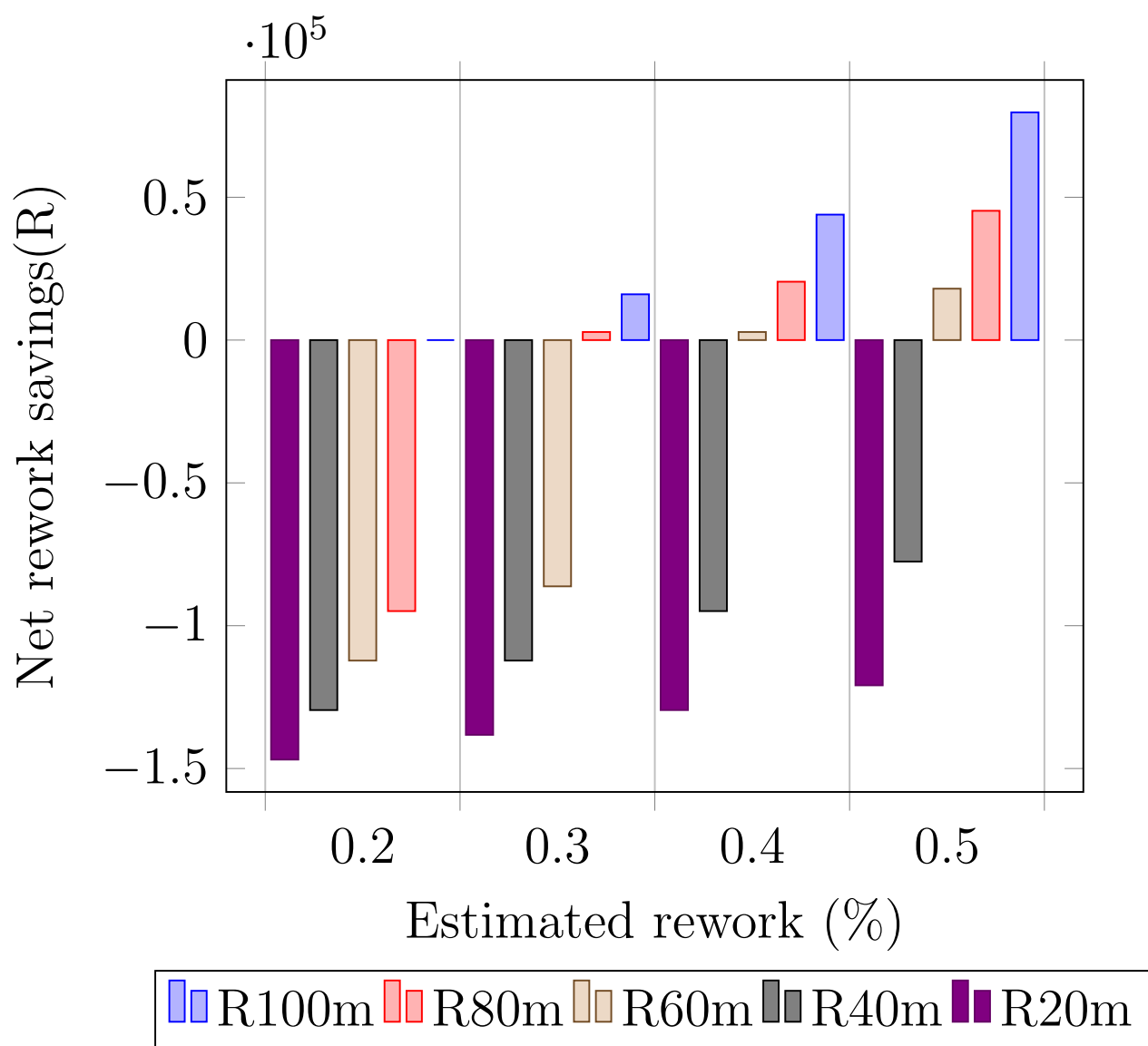

Figure 8.18: Expected savings by mitigating rework versus estimated rework values

Table 8.10: Net rework savings (losses) for various combinations of project value and expected rework percentages

\begin{tabular}{|c|c|c|c|c|c|c|c|c|}
\hline \multirow{2}{*}{$\begin{array}{c}\text { Concrete } \\
\text { works value } \\
(\mathrm{R})\end{array}$} & \multicolumn{8}{|c|}{ Expected rework percentage } \\
\hline & $4 \%$ & $3 \%$ & $2 \%$ & $1 \%$ & $0.5 \%$ & $0.4 \%$ & $0.3 \%$ & $0.2 \%$ \\
\hline \multicolumn{9}{|c|}{ Expected rework saving (loss) corresponding to concrete works value (R) } \\
\hline 100 million & 1611253 & 1174040 & 736826 & 299613 & 79729 & 43926 & 16034 & 0 \\
\hline 90 million & 1436368 & 1042876 & 649384 & 259511 & 62496 & 30273 & 9431 & (86 219) \\
\hline 80 million & 1261482 & 911712 & 561941 & 220387 & 45263 & 20436 & 2827 & $(94884)$ \\
\hline 70 million & 1086597 & 780548 & 474498 & 181264 & 29423 & 11632 & $(73222)$ & (103 548) \\
\hline 60 million & 911712 & 649384 & 387056 & 142140 & 17998 & 2827 & $(86$ 219) & (112 213) \\
\hline 50 million & 736826 & 518220 & 299613 & 79729 & 6665 & $(77555)$ & (99 216) & (120 877) \\
\hline 40 million & 561941 & 387056 & 220387 & 45263 & $(77555)$ & (94 884) & (112 213) & (129 542) \\
\hline 30 million & 387056 & 259511 & 142140 & 17998 & (99 216) & (112 213) & (125 209) & (138 206) \\
\hline 20 million & 220387 & 142140 & 45263 & $(77555)$ & (120 877) & (129 542) & (138 206) & (146 871) \\
\hline 10 million & 45263 & 17998 & $(77555)$ & (120 877) & (142 538) & (146 871) & (151 203) & (155 535) \\
\hline 1 million & 146687 & (151 203) & (155 535) & (159 867) & (162 033$)$ & (162 467) & (162 900) & (163 333) \\
\hline
\end{tabular}




\subsection{Validation interviews}

Interviews were held with participants from the construction industry. The purpose of the interviews were to gain participants' opinion regarding the following aspects of the study:

- Attributes identified under labour, management, subcontractors, and plant and equipment that lead to poor quality

- Construction errors identified as risks

- Availability of data for the cost allocation toward each risk

- Current methods used in companies for making technology investment decisions

- The usefulness of the decision model in industry

- Implementation of identified technologies on site

- Current views of contractors toward the implementation of technology

Semi-structured interviews were conducted with 5 participants from the construction industry. The participants provided their opinion on the model after the author explained the logic of the model and asked questions on the model. Table 8.11 shows the experience of the participants:

Table 8.11: Experience of the participants in the validation interviews

\begin{tabular}{cc}
\hline $\begin{array}{c}\text { Participant } \\
\text { number }\end{array}$ & $\begin{array}{c}\text { Construction } \\
\text { experience } \\
\text { (Years) }\end{array}$ \\
\hline Participant 1 & 8 \\
\hline Participant 2 & 14 \\
\hline Participant 3 & 20 \\
\hline Participant 4 & 35 \\
\hline Participant 5 & 9 \\
\hline
\end{tabular}

The participants were asked to provide their opinion on 7 open-ended questions. Table 8.12 shows the questions that were asked whilst presenting the model to the participants. 
Table 8.12: Validation questions answered by participants

\begin{tabular}{cl}
\hline Question number & \multicolumn{1}{c}{ Description } \\
\hline 1 & $\begin{array}{l}\text { Are there other attributes under labour, management, subcon- } \\
\text { tractor, and plant and equipment that are not included in the } \\
\text { model, that would also have a significant influence on concrete } \\
\text { quality? }\end{array}$ \\
\hline $\begin{array}{l}\text { After viewing the risks identified in the model, are there other } \\
\text { construction errors that also lead to concrete rework, but are not } \\
\text { included in the model computations? }\end{array}$ \\
\hline $\begin{array}{l}\text { After viewing the procedure of cost allocation for risk rework, } \\
\text { is there company data available to assist in the cost allocation } \\
\text { required in the model? }\end{array}$ \\
\hline $\begin{array}{l}\text { What methods are currently used at your company to assist in } \\
\text { technology investment decision-making? }\end{array}$ \\
$\begin{array}{l}\text { Do you think such a model could work in industry to assist in the } \\
\text { decision-making of technological investments to improve quality } \\
\text { control? }\end{array}$ \\
\hline $\begin{array}{l}\text { After explaining the current technologies and how they are used } \\
\text { to reduce rework, do you think these technologies could reduce } \\
\text { rework and be successfully implemented on site? }\end{array}$ \\
\hline $\begin{array}{l}\text { What is the general attitude of contractors toward implementing } \\
\text { new technologies in construction? }\end{array}$ \\
\hline 7
\end{tabular}

The opinions from each of the participants are discussed below. The individual participant answers are shown in Tables 9 to 13 in Appendix J.

\subsubsection{Summary of interviews}

From the interviews it was found that the participants were satisfied with the range of attributes that were identified under labour, management, subcontractors, and plant and equipment. According to the participants, most of the critical construction errors were identified. It was mentioned that cold-joints were omitted in the study as well as poor planning with regards to material volumes and equipment usage. A concern regarding the quality control of batch plants were raised. However, in this study the scope was directed towards the specific construction procedures on site. It could be reasoned that a batch plant is on site and directly involved in the construction process. In that case, further research is required to monitor the batching plant processes.

It was found that companies do not process data in a format that allow the cost of various causes of rework to be obtained. However, it could be done by processing the non-conformance slips and categorising the data. Most of the companies follow a qualitative approach when investing in technology. The use, cost and benefits of the technology is discussed and based on the findings a decision is made. One of the participants mentioned that spreadsheets with estimations are created to justify the technological investments.

All the participants agreed that the proposed system could be used in industry to assist in risk identification and technology investment decisions. Three of the participants were confident in the use of such a system. The other two agreed that it could be used, but that it would be more useful in the tendering phase of a project to assist in the calculation 
of project cost.

All the participants agreed that the identified technologies can be implemented on site to improve quality control. However, the technology should be easy to use, not very time consuming and someone should be appointed to carry the responsibilities of such a system.

Most of the participants were of the opinion that older contractors are reluctant to change and do not easily welcome technologies in construction. However, the younger generation is more innovative and keen to implement technological systems. Furthermore, barriers such as unskilled labour and the need of a body in the company to manage such a system was said to make the implementation of technology difficult. 


\section{Chapter 9}

\section{Conclusions and recommendations}

This Chapter provides a summary of the study, conclusions made regarding the study, recommendations for future research and the research limitations of the study.

\subsection{Summary}

The focus of this study was on the investment of technology to reduce rework on structural concrete elements, such as beams, columns, slabs and reinforced walls. A decision model based on risk and cost was considered to assist with the investment decision. The study was conducted at the University of Stellenbosch in South Africa. The research period spanned between January 2016 and December 2017.

Both qualitative and quantitative research methods were used in the study. As qualitative method, interviews were conducted to gain experts' knowledge on critical construction errors that lead to rework together with measures to prevent these errors. Furthermore, interviews were conducted with experts to gain their opinion on the use of the decision model in the construction industry. As quantitative method, a questionnaire survey was used to establish weights for the influence of labour, management, subcontractors, and plant and equipment attributes on the quality of construction. Furthermore, the questionnaire results assisted to quantify the impact of labour, management, subcontractors, and plant and equipment on the construction errors that were identified. Experimental tests were conducted in the Stellenbosch University laboratory to assess the laser scanner, point cloud software and image dimensioning software.

The participants to the interviews were identified through references by the supervisor of this study. They were identified as experts in the construction industry, owed to their experience on large structural concrete projects. Questionnaire participants were identified from a list of candidates that had previously attended courses presented by the Construction- and Engineering Management research department.

Descriptive and inferential statistical tools were used in this study. Descriptive statistics were used to calculate the mean and standard deviation of the questionnaire results. Furthermore, as part of descriptive statistics, histograms were generated from experimental results to estimate technology effectiveness. As part of inferential statistics, an independent two sample t-test was used to compare the means of results by contractors and 
consultants.

The information gathered from the qualitative and quantitative research methods were used to develop the decision model. The decision model uses risk and cost in the decisionmaking process.

\subsubsection{Aims and objectives}

The aim of this study was to develop a decision model to assist a contractor in the investment decision of acquiring technology to reduce rework on concrete.

To achieve the aim of this study, the following objectives were defined:

1. Interview industry experts to identify causes of concrete rework

2. Interview industry experts to identify mitigation measures to prevent rework

3. Evaluate available technologies' capability and effectiveness to improve quality control through implementation of mitigation measures

4. Quantify the risk magnitude of construction errors.

The critical construction errors that lead to rework on structural concrete were identified through literature and interviews. During the validation process, participants agreed that the important errors were addressed by the study. The critical construction errors were identified as the risks that are potentially mitigated through use of the technology systems.

Measures to mitigate the construction errors were identified through interviews. Mitigation measures provided by the interview participants that were similar, were used in the study. Identification of the mitigation measures enabled the author to identify manners in which the technology can be used to reduce rework. However, the measures presented are not the only methods of preventing rework.

Currently available technologies were evaluated through experimental tests and secondary information. The information gathered from the tests and secondary information was used to estimate the technologies' effectiveness to reduce rework. Through the validation process, participants agreed that the identified technologies could successfully be implemented on site to reduce concrete rework.

The risk magnitude of construction errors was quantified for the decision model. This was achieved through the use of questionnaire data and user input into the model.

By achieving the objectives of the study, the necessary information was obtained to develop the decision model. The model was developed based on risk and cost. From the sensitivity analyses it was concluded that the use of technology to reduce rework results in net rework savings. The technologies play an important role in the prevention of construction errors. By using the technology, quality control is automatically improved on the project due to increased quality monitoring. Net rework savings result for projects 
where the concrete works value is large (preferably $>\mathrm{R} 30$-million) with an expected rework percentage greater than $1 \%$.

During the validation interviews, participants agreed that the model could be used in the initiation stages of a project to assist management with the planning process. Furthermore, participants agreed that the model can serve as a useful tool to assist with investment decisions.

\subsection{Conclusions}

Conclusions are made regarding the decision model that was developed in the study. The primary aim of this study was to develop a decision model to assist with technology investment decisions. Therefore, the primary focus of the study is on the logic of the decision model and not on the effectiveness of the technology to reduce rework. However, the estimated technology effectiveness values are used in the model, but it is advised that effectiveness values that are representative of field conditions are obtained. This would require the testing of the technology on various real-life construction projects.

\subsubsection{Results conclusion}

Conclusions are made regarding the following aspects of the study:

- Experimental tests and effectiveness of technology

- Construction errors and category attributes

- Risk management method

- Numerical output from the decision model

- Short-term implications

- Long term implications.

This study was conducted with the expectation that technology can be used to reduce construction rework. However, various technologies had to be investigated to determine its role in reducing concrete rework. The experimental tests showed that the laser scanner and image dimensioning software are useful to improve quality control where dimension accuracy has a crucial part in the quality of the works. Technologies such as the IP camera, QR codes and concrete sensors were not tested by means of experiments. Information gathered on these technologies, together with methods such as the lessons-learned approach and human error calculations, were used to derive the technology effectiveness. It is concluded from the available information that the technologies identified in this study are useful to reduce concrete rework. This was confirmed by interview participants. However, these technologies need to be investigated under field conditions. The technology effectiveness was not the primary focus in this study; therefore, the author did not conduct thorough research on the technologies' effectiveness to reduce rework. 
By means of a questionnaire survey, information was obtained regarding the influence that the attributes of labour, management, subcontractors, and plant and equipment have on concrete quality. It was found that all the attributes identified do have an important influence on the construction quality of concrete. The labour attributes that showed the most significant impact on quality was skill level and training. The management attributes that showed the most significant influence on construction quality was supervision and communication. The subcontractor attributes that showed the most significant impact on construction quality was experience and knowledge of operations. The attributes of plant and equipment that showed the most significant influence on construction quality was the cleanliness of formwork and shutter boards.

Information regarding the impact of each category (labour, management, subcontractors, and plant and equipment) on the identified construction errors were also obtained by means of a questionnaire. It was found that each category has a significant impact on the identified construction errors. Labour has the most influential impact on concrete compaction and concrete cover. Management has the most influential impact on the position of HD bolts and concrete curing. Subcontractors have the most influential impact on steel spacing. Plant and equipment has an influential impact on delays due to breakages and poor finishing.

The model was presented to experts from the construction industry by means of an interview session. Industry experts agreed that the method used to develop the decision model could be useful in the construction industry. The model could be adopted by a company which would use the logic of the model together with their company data and expertise to adjust the model parameters. Emphasis was placed on the usefulness of the decision model to be used as a risk management tool. Participants opined that the risk identification process could be useful during the initiation stages of a project.

Scenario- and sensitivity-analyses were conducted to determine the outcomes of the model under various conditions. The results from the analyses were subject to certain conditions; therefore, it is not a representation of a specific project. However, the results assist to identify trends in the output of the model. It was concluded that the investment in technology is feasible for projects with high risk and high rework expectations. The technology investment is feasible for projects where the concrete works value exceeds $\mathrm{R}$ 30-million and the expected rework percentage is more than $1 \%$. For projects with concrete work values less than $\mathrm{R} 30$-million, the monetary value of potential rework prevented is too low to motivate the investment in technology. It was also found that the model output is sensitive for rework expectancies that are lower than $1 \%$. As the rework percentage reduces from one to zero percent, the variance for the minimum concrete works value required for the investment to be feasible increases. The reason for this is that the smaller rework percentage provides less potential rework to be prevented. Thus, less capital available to invest in the technology.

The short-term implication of the findings in this study is not yet advantageous to the construction industry. The reason therefore is that more research is required before an official decision model is established. Over the short-term, companies should be identified that are willing to implement the model and technologies on their projects. Only after 
the implementation on a number of projects, adjustments can be made to better quantify the current parameters of the model. The parameters of the model that require improved quantification are: (1) the effectiveness of technology to reduce rework and (2) the inputs used to calculate the risk magnitude. The inputs used to calculate risk magnitude can be improved through more extensive research and comparing the risk magnitudes provided by the model to that of industry expert's expectations.

The long-term implication of the findings in this study is advantageous to the construction industry. Considering the credit risk status of South Africa to sovereign debt, the author reasons that infrastructure development costs for the government are going to increase. The reason is that the government needs to borrow money from other countries at a higher risk return interest rate. Therefore, less funds are budgeted for infrastructure development. Engineering- and construction companies will have to be more competitive during the tendering phase, which means that profit margins need to be reduced. Profit margins are influenced by the cost of production and the tender price. Since the tendering prices are already competitive, construction companies need to reduce their production costs to remain competitive. The use of technology would reduce production costs by saving rework. In the long-run the model parameters would already be adjusted to provide the user with a reliable investment decision. Therefore, the user gets a reliable estimation of money that can be saved from preventing rework by means of technology. Furthermore, the use of technology in construction will motivate the development of more innovative technologies in construction.

\subsection{Recommendations}

In this Section the author discusses various recommendations from the study. The recommendations pertain toward the decision model that was developed. It aims to propose actions that need to be taken by future researchers to ensure the success of the decision model. Furthermore, a recommendation is made regarding an alternative that is best supported by the study.

\subsubsection{Decision model}

Various recommendations were identified to improve the reliability of the decision model.

The decision model relies on the input of one user to rate the attributes of labour, management, subcontractors, and plant and equipment. The user might incorrectly estimate the ratings for these attributes, which would result in the incorrect calculation of risk magnitudes. As a recommendation, the user should be enabled to repeat the attribute ratings based on different expected scenarios. The model should then calculate the average risk magnitude of each risk based on the different scenarios. Furthermore, various users with varying experiences should be enabled to rate the attributes of the project so that the average attribute ratings are used in the risk calculation.

The model currently relies on the assumption that the performance expected from labour, management, subcontractors, and plant and equipment is related to some likelihood that 
construction errors would occur. Future research is required to determine the relationship between the expected performance and likelihood. This can be achieved by interviews with experts, analysing previous projects or by observing the cause of construction error occurrences on a project.

The model currently assigns a likelihood value to each risk. The value was derived from the weighted average of each category's attribute ratings. For example, the likelihood assigned to incorrect concrete cover and compaction are equal since they are caused by labour. It is recommended that the different attributes that increase the likelihood of each risk be investigated. Furthermore, the risks can be a result of more than one category. Therefore, the risks can be quantified as a function of more than one category.

The model relies on the user to allocate the cost effect of each of the risks if rework was caused by the risk. It is suggested that the user simulate different scenarios of the cost allocation process. This could also be achieved by developing probability density functions (PDFs) for the cost of each risk. Through use of a simulation method, such as the Monte Carlo analysis, the model then estimates the most probable scenario. It is recommended that PDFs for the cost effect of each risk in concrete construction.

In this study, the technologies were not extensively evaluated to determine its effectiveness to reduce rework. The rework reduction effectiveness that was determined in this study is not based on field conditions. It is recommended that companies implement these technologies on several projects to assess its effectiveness to reduce construction rework. The methods used in this study (laboratory tests, human error calculations and lessons learned effectiveness) might not support the effectiveness of the technologies on site. The author was limited to a given time span; therefore, the effectiveness on real-life projects was not determined.

During the interviews that form part of the validation process of the study, concerns were raised regarding the omission of quality control on batch plants that are on site. It can be reasoned that batch plants are directly involved in the construction process. However, the quality control at the batch plant was not considered for this study. It is recommended that the methodology followed to develop the decision model be applied to that of a batch plant environment. Other technologies should be investigated which could be useful during the batch plant processes.

\subsection{Research limitations}

The author recommends that future researchers who aim to investigate the various construction errors, should spend enough time on a construction site to improve the understanding of construction site dynamics. An improved understanding would be obtained of the rate at which processes occur on site, how the quality is controlled and how technology could be implemented during this process.

From personal experience it was found that a construction site is very dynamic and there is little time to implement incentives that are beyond the scope of works. Therefore, 
only technology that does not intervene with the dynamic flow of works would be easy to implement on site. In other words, technology should not be used if it is difficult to implement and use on site.

This study made use of interviews and questionnaires. Interviews provide valuable information that are normally gained through years of experience. Therefore, it is recommended that interviews are conducted with more participants to gain an in-depth understanding of the investigation. However, interviews require a fair amount of effort. It is difficult to find participants with enough experience that are willing to participate in interviews.

The technologies in this study were evaluated from information available on the technologies and results from experimental tests. It is recommended that the technologies are implemented on a construction site to evaluate its effectiveness to reduce rework. In this study the author was limited by time and money to implement the technologies on a construction site.

An alternative recommendation is made to reduce construction rework without the use of technology. The attribute ratings of labour, management, subcontractors, and plant and equipment are used in this study to predict the likelihood of risks on a project. Therefore, it is reasoned that by improving the performance of these attributes, the construction rework could be reduced.

For example, this could be achieved for labour as follows:

- Give labourers adequate training to improve their skills

- Appoint labourers who already have a good skill level

- Give labour performance bonuses to improve their motivation

- Use less casual labour by constantly using the same workforce (Possible difficulties due to tender requirements).

Considering the aforementioned, an investigation is required to determine the costs and benefits of improving labour, management, subcontractors, and plant and equipment on a project. The net benefit (loss) should then be compared that of using technology to reduce construction risk. 


\section{Bibliography}

3D Laser mapping (2016). 3-IN-1 MOBILE MAPPING SYSTEM [Online]. Retrieved August 28, 2017.

URL: $\quad$ http://www.3dlasermapping.com/wp-content/uploads/2016/05/ROBINdatasheet-May-2016

3D Systems Inc. (n.d.). 3D scanners - A guide to 3D scanner technology [Online]. Retrieved August 28, 2017.

URL: http://www.rapidform.com/3d-scanners

Addis, B. (2005). Fundamentals of Concrete, Midrand: Cement and Concrete Institution

Akinci, B., Boukamp, F., Gordon, C., Huber, D., Lyons, C. and Park, K. (2006). A formalism for utilization of sensor systems and integrated project models for active construction quality control, 15: 124-138.

Alexander, M. G., Ballim, Y. and Stanish, K. (2008). A framework for use of durability indexes in performance-based design and specifications for reinforced concrete structures, Materials and Structures 41(5): 921-936.

Alexander, M. G., Santhanam, M. and Ballim, Y. (2011). Durability design and specification for concrete structures, International Journal of Advances in Engineering Sciences and Applied Mathematics 2(3): 95-105.

URL: http://www.springerlink.com/content/l43787667435063p/

Angelucci, M. (2016). Site visit to Stellenbosch WWTW.

Aurvis R\&D (2014). PixelScanner [Online]. Retrieved August 28, 2017.

URL: http://www.aurvis.com/pixelscanner.html

Ayandibu, O. G. (2010). SOCIO-ECONOMIC OBJECTIVES IN THE CONSTRUCTION OF THE.

Ballard, G., Howell, G. and Member, . (1998). Shielding Production: Essential Step in Production Control, 124(February): 11-17.

Basheer, L., Kropp, J. and Cleland, D. J. (2001). Assessment of the durability of concrete from its permeation properties: A review, Construction and Building Materials 15(23): $93-103$.

Bell, J. (2005). Doing Your Research Project, pp. 152-164. 
Best, J. (1981). Research in Education, Prentice Hall: New Delhi 4.

Boukamp, F. and Akinci, B. (2007). Automated processing of construction specifications to support inspection and quality control, Automation in Construction 17(1): 90-106.

Bowden, S., Dorr, A., Thorpe, T. and Anumba, C. (2006). Mobile ICT support for construction process improvement, Automation in Construction 15(5): 664-676.

Brick House Security (2016). What is an internet camera [Online]. Retrieved August 28, 2017.

URL: http://www.brickhousesecurity.com/category/video+surveillance+security+cameras

Burke, R. (2007). Project Management Techniques, Burke Publishing .

Busby, J. (1999). An assessment of post-project reviews, Project Management Journal (30): 23-29.

Business Dictionary (2013). Quality [Online]. Retrieved August 28, 2017.

URL: www.businessdictionary.com/definition/quality.html

Cheng, T. and Teizer, J. (2013). Real-time resource location data collection and visualization technology for construction safety and activity monitoring applications, $A u$ tomation in Construction 34: 3-15.

URL: http://dx.doi.org/10.1016/j.autcon.2012.10.017

CIDB: South Africa (2016). CIDB Mandate [Online]. Retrieved August 28, 2017.

URL: http://www.cidb.org.za/Pages/default.aspx

Construction Industry Institute (2007). Implementation of Lessons Learned Programs.

Department of Labour (2002). Investigation report into the Injaka Bridge Collapse of 6 July 1998 [Online]. Retrieved August 28, 2017.

URL: http://www.projectpro.co.za/ProjectParade/InjakaLessonLearned/

Direct Dimensions (2016). Rapid solutions to 3D problems [Online]. Retrieved August 28, 2017.

URL: http://www.dirdim.com/lm_everything.htm

Du, J. C. and Teng, H. C. (2007). 3D laser scanning and GPS technology for landslide earthwork volume estimation, Automation in Construction 16(5): 657-663.

Duncan, J. M., Thorpe, B. and Sunmer, P. (1990). Quality assurance in Construction, Gower Publishing Company Limited.

Elbeltagi, E. (1987). Construction Site Layout Planning, Journal of Computing in Civil Engineering pp. 1-18.

URL: $\quad$ http://drahmedelyamany.weebly.com/uploads/7/0/1/0/7010103/sitelayout_dremad.pdf

Emailitis (2012). IP Camera Network Setup [Online]. Retrieved August 28, 2017.

URL: http://www.ip-security.co.uk/further-info/ip-camera-network-setup/ 
Faro (2016). Faro Focus 3D [Online]. Retrieved August 28, 2017.

URL: http://www.faro.com/en-us/products/3d-surveying/faro-focus3d/overview

Farrington, J. J. and Ledbetter, W. B. (1992). Causes of quality deviations in design and construction, 118(1): 34-49.

Fellows, R. and Liu, A. (2008). Research methods for construction, Blackwell Science: United Kingdom 3rd.

Ferrada, X., Núñez, D., Neyem, A., Serpell, A. and Sepúlveda, M. (2016). A Lessonslearned System for Construction Project Management: A Preliminary Application, Procedia - Social and Behavioral Sciences 226(October 2015): 302-309.

URL: http://dx.doi.org/10.1016/j.sbspro.2016.06.192

FIDIC (n.d.). Quality of Construction [Online]. Retrieved August 28, 2017.

URL: http://www1.fidic.org

Ghani, U., Shabbir, F. and Khan, K. M. (2006). Change in concrete properties due to lack of curing.

Golparvar-fard, M., Bohn, J., Teizer, J., Savarese, S. and Peña-mora, F. (2011). Automation in Construction Evaluation of image-based modeling and laser scanning accuracy for emerging automated performance monitoring techniques, Automation in Construction 20(8): 1143-1155.

URL: http://dx.doi.org/10.1016/j.autcon.2011.04.016

Gomm, R. (2008). Social Research Methodology: A critical introduction, Palgrave Macmillan 2.

Hallermann, N. (2015). Vision-based monitoring of heritage monuments by Unmanned Aerial Systems ( UAS ) for detailed inspection and high-accurate survey of structures.

Harris, F. and McCaffer, R. (1995). Modern Construction Management, Cambridge University Press (4th Edition).

Harris, F., McCaffer, R. and Edum-Fotwe, F. (2006). Modern Construction Management, Blackwell Publishing.

Harvey, A. and Kagan (1990). Practical Quality - Controlled Construction, ASCE .

Harwin, S. and Lucieer, A. (2012). Assessing the accuracy of georeferenced point clouds produced via multi-view stereopsis from Unmanned Aerial Vehicle (UAV) imagery, Remote Sensing 4(6): 1573-1599.

Impinj, I. (2016). About RFID [Online]. Retrieved August 28, 2017.

URL: http://www.impinj.com/resources/about-rfid/rfid-standards

ISO 9000 (1994). International Organisation for Standardization, Technical report.

IVC (n.d.). Remote Construction Site Monitoring [Online]. Retrieved August 28, 2017.

URL: http://www.ivcco.com/application/App Note - Remote Construction Site Monitoring.pdf 
Jaselskis, E., Anderson, M. R., Jahren, C. T., Rodriguez, Y. and Njos, S. (1995). Radiofrequency identification applications in construction industry, Journal of Construction Engineering and Management 121(2): 189- 196.

Jergeas, G. F. and Ruwanpura, J. (2010). Why Cost and Schedule Overruns on Mega Oil Sands Projects?, Practice Periodical on Structural Design and Construction 15(1): 4043.

Jugdev, K. (2012). Learning from Lessons Learned: Project Management Research Program, American Journal of Economics and Business Administration 4(1): 13-22.

Kaplan Inc. (2015). CFA level 1 Quantitative Methods.

Kim, M., Sohn, H. and Chang, C. (2014). Automated dimensional quality assessment of precast concrete panels using terrestrial laser scanning, Automation in Construction 45: $163-177$.

Kirwan, B., Kennedy, R. and Taylor-adams, S. (1997). The validation of three Human Reliability Quantification techniques - THERP , HEART and JHEDI : Part II - Results of validation exercise, $2(\mathrm{I})$.

Kothari, C. (2004). Research Methodolgy: Methods and Techniques, New Delhi, New Age .

Kotnour, T. (1999). A learning framework for project management, Project Management Journal 30: 32-38.

Laerd Statistics (2013). Independant t-test for two samples [Online]. Retrieved August 28, 2017.

URL: https://statistics.laerd.com/statistical-guides/independent-t-test-statisticalguide.php

Laser Design (2016). 3D scanning - Hard work that looks like magic [Online]. Retrieved August 28, 2017.

URL: http://www.laserdesign.com/what-is-3d-scanning

Leedy, P. and Ormrod, J. (2010). Practical Research: Planning and design, Macmillan Publishing Company: New York .

Lombard, A. (2011). Decision making between hybrid and in-situ concrete construction in South Africa.pdf, SUNScholar Research Repository .

Long, N. D., Ogunlana, S., Quang, T. and Lam, K. C. (2004). Large construction projects in developing countries: A case study from Vietnam, International Journal of Project Management 22(7): 553-561.

Love, P., Ackermann, F., Carey, B., Morrison, J., Ward, M. and Park, A. (2016). The praxis of rework mitigation in construction, Journal of Management in Engineering 32: $0-10$.

Love, P. and Li, H. (2000). Quantifying the causes and costs of rework in construction, Construction Management and Economics 18. 
Mackechnie, J. R. and Alexander, M. G. (2002). Durability Predictions Using Early Age Durability Index Testing, (1993): 1-9.

Mani, G. F., Feniosky, P. M. and Savarese, S. (2009). D4AR-A 4-dimensional augmented reality model for automating construction progress monitoring data collection, processing and communication, Electronic Journal of Information Technology in Construction 14(June): 129-153.

Martin, W. J. (n.d.). Chapter 8 - Concrete.

URL: http://www.nra.co.za/content/Chapter_8.pdf

Minitab Inc. (2015). Understanding Hypothesis Tests: Significance Levels (Alpha) and $\mathrm{P}$ values in Statistics [Online]. Retrieved August 28, 2017.

URL: $\quad$ http://blog.minitab.com/blog/adventures-in-statistics-2/understandinghypothesis-tests:-significance-levels-alpha-and-p-values-in-statistics

Mitropoulos, P. and Memarian, B. (2012). A framework of teamwork attributes affecting worker safety, Construction Research Congress 2012 ASCE.

Muigai, R., Moyo, P. and Alexander, M. (2012). Durability design of reinforced concrete structures: a comparison of the use of durability indexes in the deemed-to-satisfy approach and the full-probabilistic approach, Materials and Structures 45(8): 1233-1244. URL: http://www.springerlink.com/index/10.1617/s11527-012-9829-y

Nganga, G., Alexander, M. and Beushausen, H. (2013). Practical implementation of the durability index performance-based design approach, Construction and Building Materials 45: 251-261.

URL: http://dx.doi.org/10.1016/j.conbuildmat.2013.03.069

Parvin, R. (2013). Benefits of implementing RFID in Supply Chain Management, RFID Arena .

URL: http://www.rfidarena.com/2013/11/14/benefits-of-implementing-rfid-in-supplychain-management.aspx

Pauley Creative (2017). 10 Ways construction companies can use QR codes [Online]. Retrieved August 28, 2017.

URL: http://www.pauleycreative.co.uk/2011/04/10-ways-construction-companies-canuse-qr-codes/

PMI (2010). A Guide to Project Management Body of Knowledge, 4th edn.

Price Check (2017). Price check IP cameras [Online]. Retrieved August 28, 2017.

URL: https://www.pricecheck.co.za/categories/8819/IP+Cameras

Raken (2016). The \#1 Construction App for daily reporting [Online]. Retrieved August 28, 2017.

URL: https://www.rakenapp.com/

Ronne, P. D. (2006). Variation in Cover to Reinforcing, Local and International Trends .

Ropraz, F. (2008). Using RFID for Supply Chain Management, University of Freiburg Schweiz . 
Rwelamila, P. D. and Wiseman, G. T. (1995). Concrete quality management: A research study of the general contractor in South Africa., Construction and Building Materials .

Sabbaghi, A. and Vaidyanathan, G. (2008). Effectiveness and efficiency of RFID technology in supply chain management: Strategic values and challenges, Journal of Theoretical and Applied Electronic Commerce Research 3(2): 71-81.

Sangregario, J. (2012). Wireless sensing system for intelligent concrete curing, Michigan State University .

SANS 1200 (1981). SANS 1200: South African National Standards 1200.

SANS 2001-CC1 (2007). SANS 2001-CC1 : 2007 SOUTH AFRICAN NATIONAL STANDARD Construction works Part CC1 : Concrete works ( structural ).

Schindler, M. (2003). Harvesting project knowledge: A review of project learning methods and success factors, Int. J. Proj. Manage. 21: 219-228.

Scott, A. N. (2004). PhD Thesis, 1(University of Cape Town).

Shammas-Toma, M., Seymour, D. E. and Clark, L. (1996). The effectiveness of formal quality management systems in achieving the required cover in reinforced concrete, Construction Management and Economics 14(February 2015): 353-364.

Smallwood, J. (2000). A Study of the Relationship between Occupational Health and Safety, Labour Productivity and Quality in the South African Construction Industry, Unpublished PhD (Construction Management) Thesis, Department of Construction Management, University of Port Elizabeth $\mathbf{1}$.

Smallwood, J. (2012). Construction, Engineering and Public works inspection 2012 Conference, number January, pp. 20-21.

Smith, W. (2010). Distribution of Basic Variables in Reinforcing Concrete, Stellenbosch: University of Stellenbosch.

Solomons, W. J. (2014). Investigating the impact of site activities and conditions on concrete quality of in-situ and precast construction methods, (December).

Sood, A. (2006). Some pages of this thesis may have been removed for copyright restrictions ., Aston University p. 333.

Structural Health Systems, I. (2015). Concrete Sensors [Online]. Retrieved August 28, 2017.

URL: http://www.concretesensors.com/

Surface and Edge (2011). How the Laser Scanner Works [Online]. Retrieved August 28, 2017.

URL: http://www.surfaceandedge.com/technology/how-the-laser-scanner-works

Tabassi, A., Ramli, M. and Baker, A. (2011). Effect of training and motivation practices on teamwork improvement and task efficiency, International Journal of Project Management $\mathbf{3 0 .}$ 
TechTarget (2016). What is IP camera? [Online]. Retrieved August 28, 2017.

URL: http://whatis.techtarget.com/definition/IP-camera

The Transtec Group, I. (2015). Command center concrete temperature and maturity meters [Online]. Retrieved August 28, 2017.

URL: http://www.maturitycentral.com/

Toor, S. u. R. and Ogunlana, S. O. (2008). Critical COMs of success in large-scale construction projects: Evidence from Thailand construction industry, International Journal of Project Management 26(4): 420-430.

United Parcel Service of America (2005). Solutions in the Supply Chain System: An Overview of the Promises and Pitfalls, Solutions in the Supply chain .

Walliman, N. (2005). Your research project, Sage Publications London 2nd.

Wang, L.-C. (2008). Enhancing construction quality inspection and management using RFID technology, Automation in Construction 17(4): 467-479.

Wang, Q., Cheng, J. C. P. and Sohn, H. (2015). Automated Quality Inspection of Precast Concrete Elements with Irregular Shapes Using Terrestrial Laser Scanner and BIM Technology, Proceedings of the International Symposium on Automation and Robotics in Construction 32.

Williams, J. (1988). A data-based method for assessing and reducing human error to improve operational performance, In Human Factors and Power Plants .

Williams, T. (2008). How do organizations learn lessons from projects - and do they?, IEEE Transactions on Engineering Management 55(2): 248-266.

Yuan, J. and Skaik, S. (2008). Lessons learned practices in the UAE construction industry, $C I B$.

Zairi, M. (1991). Total Quality Management for Engineers, Woodhead publishers .

Zhang, D., Haas, C., Goodrum, P., Caldas, C. and Granger, R. (2012). Construction small-projects rework reduction for capital facilities., American Society of Civil Engineers. 


\section{Appendices}




\section{A The importance of curing}

A study by Ghani, Shabbir and Khan (2006) analysed the strength of concrete when cured at 3,7 and 28 days and then followed the same procedure when curing the concrete for 2, 5 and 20 days. Figure 1 shows the reduction in the strength of concrete samples that were not cured sufficiently.

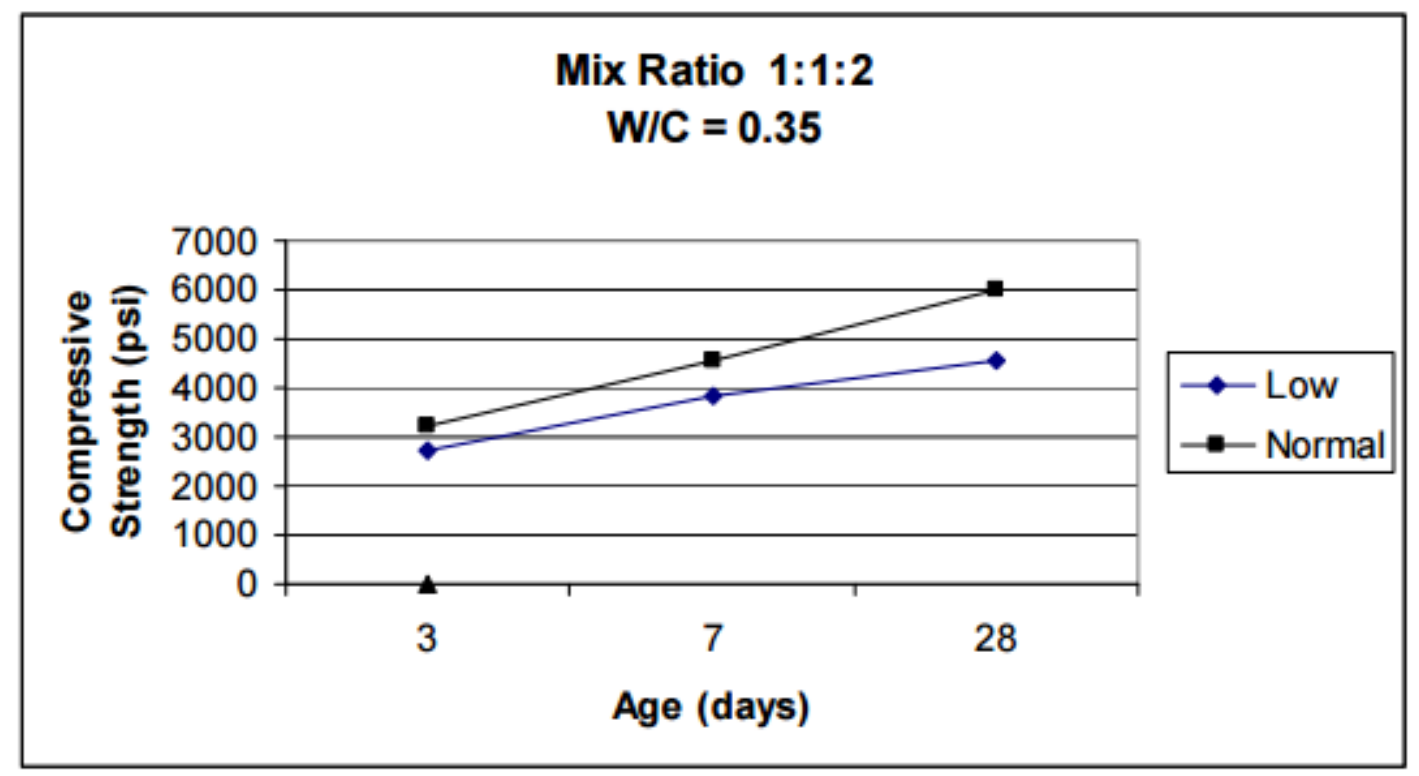

Figure 1: Graph showing the compressive strength of concrete over a given time period

Poor curing results in a permanently defective micro-structure. The size of pores and the total porosity have a significant influence on the durability of concrete since they affect the rate of ingress of aggressive agents. Experiments by Sood (2006) showed that the penetration volume of moisture into concrete increases significantly as the pore diameter increases.

When curing concrete, it is also important to monitor the carbonation rate. At a high Relative Humidity $(\mathrm{RH})$ most pores are full of water and the diffusion of $\mathrm{CO}_{2}$ into the paste becomes restricted. For a low $\mathrm{RH}$ there is insufficient water in the pores for $\mathrm{CO}_{2}$ to form carbonic acid. Carbonation is described by Sood (2006) as the neutralisation of porous cementitious materials by acidic gases such as $\mathrm{CO}_{2}$, which diffuse into the structure. The rate of carbonation depends on the moisture content and the $\mathrm{RH}$ of the ambient medium. Carbonation results in an increase of strength achieved by the concrete. The problem identified with carbonation is that it reduces the $\mathrm{pH}$ of the cement. This has an undesirable influence on the protection of the embedded steel in the presence of oxygen and water. It is also important to monitor the temperature of the concrete. Changes in temperature can lead to thermal stress and thermal shrinkage cracks (Sood, 2006). Figure 2 shows the carbonation rate for a relative humidity (\%). 


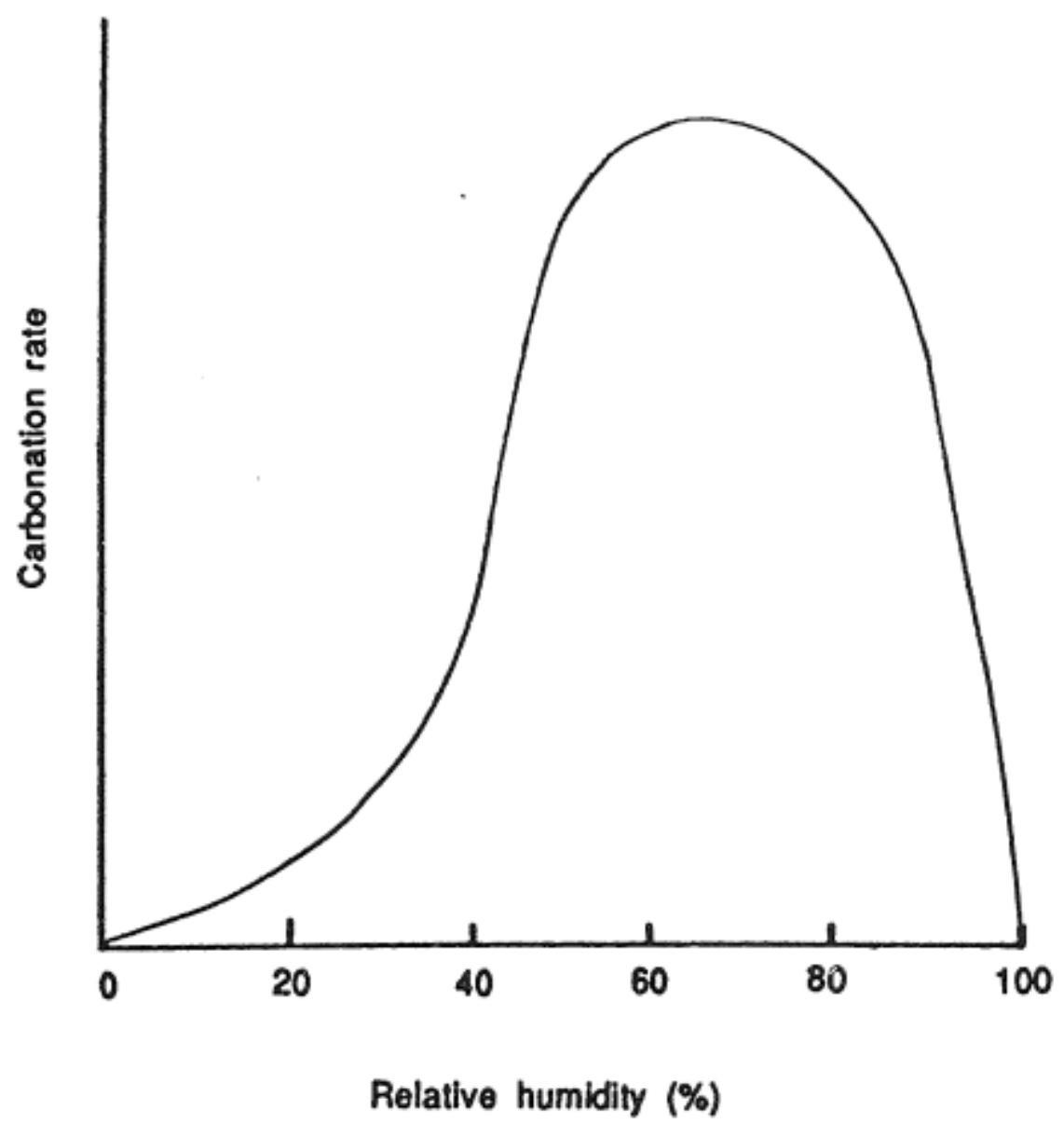

Figure 2: Graph showing the carbonation rate as the relative humidity changes

\section{B Evaluation forms for technologies}

Table 1: Evaluation criterion used to assess the capability of a technological system

\begin{tabular}{l|l|l}
\hline $\begin{array}{l}\text { Criteria to evaluate } \\
\text { capability }\end{array}$ & Yes/no & Motivation \\
\hline $\begin{array}{l}\text { Can the technological } \\
\text { system be used to } \\
\text { measure the spacing } \\
\text { between reinforcing? }\end{array}$ & Yes & $\begin{array}{l}\text { Point cloud data } \\
\text { makes it possible to } \\
\text { dimension objects. }\end{array}$ \\
\hline $\begin{array}{l}\text { Can the system be } \\
\text { used to monitor the } \\
\text { vibration process? }\end{array}$ & No & $\begin{array}{l}\text { No real time feed nor } \\
\text { video/imagery footage. }\end{array}$ \\
$\begin{array}{l}\text { Can the system be } \\
\text { used to determine } \\
\text { whether the vibration } \\
\text { is adequate and that } \\
\text { the correct compaction } \\
\text { is obtained? }\end{array}$ & No & $\begin{array}{l}\text { No real time feed nor } \\
\text { video/imagery footage. }\end{array}$ \\
\hline
\end{tabular}




\begin{tabular}{|c|c|c|}
\hline $\begin{array}{l}\text { Can the system be } \\
\text { used to monitor the } \\
\text { measures taken to } \\
\text { prevent grout loss? }\end{array}$ & No & $\begin{array}{l}\text { No real time feed nor } \\
\text { video/imagery footage. }\end{array}$ \\
\hline $\begin{array}{l}\text { Can the system be } \\
\text { used to check whether } \\
\text { cover blocks have been } \\
\text { inserted? }\end{array}$ & Yes & $\begin{array}{l}\text { Good quality scans } \\
\text { make it possible to see } \\
\text { the cover blocks. }\end{array}$ \\
\hline $\begin{array}{l}\text { Can the system be } \\
\text { used to measure the } \\
\text { dimensions of the cover } \\
\text { blocks? }\end{array}$ & Yes & $\begin{array}{l}\text { Good quality scans } \\
\text { make it possible to } \\
\text { measure the cover } \\
\text { block dimensions. }\end{array}$ \\
\hline $\begin{array}{l}\text { Can the system be } \\
\text { used to check whether } \\
\text { actions have been } \\
\text { engaged in to prevent } \\
\text { the kicking of } \\
\text { formwork? }\end{array}$ & No & $\begin{array}{l}\text { Scans will show the } \\
\text { presence of blocks and } \\
\text { bracing that prevent } \\
\text { the kicking of } \\
\text { formwork. System will } \\
\text { not be as effective as a } \\
\text { camera. }\end{array}$ \\
\hline $\begin{array}{l}\text { Can the system be } \\
\text { used to check whether } \\
\text { bleeding occurs after } \\
\text { power floating the } \\
\text { concrete? }\end{array}$ & No & $\begin{array}{l}\text { No real time feed nor } \\
\text { video/imagery footage. }\end{array}$ \\
\hline $\begin{array}{l}\text { Can the system be } \\
\text { used to measure the } \\
\text { slump and check the } \\
\text { consistency of the } \\
\text { concrete mix? }\end{array}$ & No & $\begin{array}{l}\text { No real time feed nor } \\
\text { video/imagery footage. }\end{array}$ \\
\hline $\begin{array}{l}\text { Can the technology be } \\
\text { used to inspect the } \\
\text { cleanliness of the } \\
\text { equipment or to } \\
\text { monitor the cleaning of } \\
\text { equipment on site? }\end{array}$ & No & $\begin{array}{l}\text { No real time feed nor } \\
\text { video/imagery footage. }\end{array}$ \\
\hline $\begin{array}{l}\text { Can the system be } \\
\text { used to determine } \\
\text { when the curing } \\
\text { process is adequate to } \\
\text { ensure a durable } \\
\text { concrete? }\end{array}$ & No & $\begin{array}{l}\text { System cannot provide } \\
\text { information regarding } \\
\text { the curing process. }\end{array}$ \\
\hline $\begin{array}{l}\text { Can the system be } \\
\text { used to monitor the } \\
\text { process of applying } \\
\text { curing principles? }\end{array}$ & No & $\begin{array}{l}\text { Could possibly check } \\
\text { that curing has } \\
\text { commenced, but will } \\
\text { not be effective. }\end{array}$ \\
\hline $\begin{array}{l}\text { Can the system be } \\
\text { used to determine the } \\
\text { lap-lengths of } \\
\text { reinforcing? }\end{array}$ & No & $\begin{array}{l}\text { Good quality scans } \\
\text { make it possible to } \\
\text { measure the } \\
\text { dimensions. }\end{array}$ \\
\hline $\begin{array}{l}\text { Can the system be } \\
\text { used to check whether } \\
\text { the pull-out bars are } \\
\text { correct? }\end{array}$ & Yes & $\begin{array}{l}\text { Pull-out bar levels } \\
\text { determined from scan. }\end{array}$ \\
\hline
\end{tabular}




\begin{tabular}{l|l|l}
\hline $\begin{array}{l}\text { Does the technological } \\
\text { option provide footage } \\
\text { to identify surface } \\
\text { deformations on } \\
\text { shutter boards? }\end{array}$ & Yes & $\begin{array}{l}\text { Good quality scan will } \\
\text { enable user to identify } \\
\text { the correct formwork. }\end{array}$ \\
\hline $\begin{array}{l}\text { Can the system be } \\
\text { used to ensure that the } \\
\text { equipment and } \\
\text { machinery is } \\
\text { adequately cleaned } \\
\text { before use? }\end{array}$ & No & $\begin{array}{l}\text { No real time feed nor } \\
\text { video/imagery footage. }\end{array}$ \\
\hline $\begin{array}{l}\text { Can the system be } \\
\text { used to determine the } \\
\text { correct location of the } \\
\begin{array}{l}\text { Holding down (HD) } \\
\text { bolts? }\end{array}\end{array}$ & Yes & $\begin{array}{l}\text { Accurate dimensioning } \\
\text { enables system to } \\
\text { determine position of }\end{array}$ \\
\hline $\begin{array}{l}\text { Can the system be } \\
\text { used for material } \\
\text { handling on-site? }\end{array}$ & No bolts. \\
\hline $\begin{array}{l}\text { Can the system be } \\
\text { used to check the } \\
\text { dimensions and }\end{array}$ & Yes \\
$\begin{array}{l}\text { alignment of } \\
\text { formwork? }\end{array}$ & & $\begin{array}{l}\text { Provides no } \\
\text { information regarding } \\
\text { material. }\end{array}$ \\
\hline
\end{tabular}

Table 2: Evaluation criterion used to assess the capability of image dimensioning software

\begin{tabular}{l|l|l}
\hline $\begin{array}{l}\text { Criteria to evaluate } \\
\text { capability }\end{array}$ & Yes/no & Motivation \\
\hline $\begin{array}{l}\text { Can the technological } \\
\text { system be used to } \\
\text { measure the spacing } \\
\text { between reinforcing? }\end{array}$ & Yes & $\begin{array}{l}\text { Accurate dimensioning } \\
\text { when used correctly. }\end{array}$ \\
\hline $\begin{array}{l}\text { Can the system be } \\
\text { used to monitor the } \\
\text { vibration process? }\end{array}$ & No & $\begin{array}{l}\text { Only used for } \\
\text { dimensioning purposes. }\end{array}$ \\
\hline $\begin{array}{l}\text { Can the system be } \\
\text { used to determine } \\
\text { whether the vibration } \\
\text { is adequate and that } \\
\text { the correct compaction } \\
\text { is obtained? }\end{array}$ & No & $\begin{array}{l}\text { Only used for } \\
\text { dimensioning purposes. }\end{array}$ \\
\hline $\begin{array}{l}\text { Can the system be } \\
\text { used to monitor the } \\
\text { measures taken to } \\
\text { prevent grout loss? }\end{array}$ & No & $\begin{array}{l}\text { Only used for } \\
\text { dimensioning purposes. }\end{array}$ \\
\hline $\begin{array}{l}\text { Can the system be } \\
\text { used to check whether } \\
\text { cover blocks have been } \\
\text { inserted? }\end{array}$ & No & $\begin{array}{l}\text { Only used for } \\
\text { dimensioning purposes. }\end{array}$ \\
\hline
\end{tabular}




\begin{tabular}{|c|c|c|}
\hline $\begin{array}{l}\text { Can the system be } \\
\text { used to measure the } \\
\text { dimensions of the cover } \\
\text { blocks? }\end{array}$ & Yes & $\begin{array}{l}\text { Software can measure } \\
\text { the dimensions of cover } \\
\text { blocks, but it would be } \\
\text { difficult to do the } \\
\text { calibration. }\end{array}$ \\
\hline $\begin{array}{l}\text { Can the system be } \\
\text { used to check whether } \\
\text { actions have been } \\
\text { engaged in to prevent } \\
\text { the kicking of } \\
\text { formwork? }\end{array}$ & No & $\begin{array}{l}\text { Only used for } \\
\text { dimensioning purposes. }\end{array}$ \\
\hline $\begin{array}{l}\text { Can the system be } \\
\text { used to check whether } \\
\text { bleeding occurs after } \\
\text { power floating the } \\
\text { concrete? }\end{array}$ & No & $\begin{array}{l}\text { Only used for } \\
\text { dimensioning purposes. }\end{array}$ \\
\hline $\begin{array}{l}\text { Can the system be } \\
\text { used to measure the } \\
\text { slump and check the } \\
\text { consistency of the } \\
\text { concrete mix? }\end{array}$ & Yes & $\begin{array}{l}\text { Software will be able } \\
\text { to measure the slump } \\
\text { accurately. }\end{array}$ \\
\hline $\begin{array}{l}\text { Can the technology be } \\
\text { used to inspect the } \\
\text { cleanliness of the } \\
\text { equipment or to } \\
\text { monitor the cleaning of } \\
\text { equipment on site? }\end{array}$ & No & $\begin{array}{l}\text { Only used for } \\
\text { dimensioning purposes. }\end{array}$ \\
\hline $\begin{array}{l}\text { Can the system be } \\
\text { used to determine } \\
\text { when the curing } \\
\text { process is adequate to } \\
\text { ensure a durable } \\
\text { concrete? }\end{array}$ & No & $\begin{array}{l}\text { Only used for } \\
\text { dimensioning purposes. }\end{array}$ \\
\hline $\begin{array}{l}\text { Can the system be } \\
\text { used to monitor the } \\
\text { process of applying } \\
\text { curing principles? }\end{array}$ & No & $\begin{array}{l}\text { Only used for } \\
\text { dimensioning purposes. }\end{array}$ \\
\hline $\begin{array}{l}\text { Can the system be } \\
\text { used to determine the } \\
\text { lap-lengths of } \\
\text { reinforcing? }\end{array}$ & Yes & $\begin{array}{l}\text { Only used for } \\
\text { dimensioning purposes. }\end{array}$ \\
\hline $\begin{array}{l}\text { Can the system be } \\
\text { used to check whether } \\
\text { the pull-out bars are } \\
\text { correct? }\end{array}$ & No & $\begin{array}{l}\text { Only used for } \\
\text { dimensioning purposes. }\end{array}$ \\
\hline $\begin{array}{l}\text { Does the technological } \\
\text { option provide footage } \\
\text { to identify surface } \\
\text { deformations on } \\
\text { shutter boards? }\end{array}$ & No & $\begin{array}{l}\text { Only used for } \\
\text { dimensioning purposes. }\end{array}$ \\
\hline
\end{tabular}




\begin{tabular}{l|l|l}
\hline $\begin{array}{l}\text { Can the system be } \\
\text { used to ensure that the } \\
\text { equipment and } \\
\text { machinery is } \\
\text { adequately cleaned } \\
\text { before use? }\end{array}$ & No & $\begin{array}{l}\text { Only used for } \\
\text { dimensioning purposes. }\end{array}$ \\
\hline $\begin{array}{l}\text { Can the system be } \\
\text { used to determine the } \\
\text { correct location of the }\end{array}$ & Yes & $\begin{array}{l}\text { Accurate dimensioning } \\
\text { enables system to } \\
\text { detding down (HD) } \\
\text { bolts? }\end{array}$ \\
$\begin{array}{l}\text { Can the system be } \\
\text { used for material } \\
\text { handling on-site? }\end{array}$ & No bolts. \\
\hline $\begin{array}{l}\text { Can the system be } \\
\text { used to check the } \\
\text { dimensions and } \\
\text { alignment of } \\
\text { formwork? }\end{array}$ & No & $\begin{array}{l}\text { Provides no } \\
\text { information regarding } \\
\text { material. }\end{array}$ \\
\hline
\end{tabular}

Table 3: Evaluation criterion used to assess the capability of the internet protocol cameras

\begin{tabular}{l|l|l}
\hline $\begin{array}{l}\text { Criteria to evaluate } \\
\text { capability }\end{array}$ & Yes/no & Motivation \\
\hline $\begin{array}{l}\text { Can the technological } \\
\text { system be used to } \\
\text { measure the spacing } \\
\text { between reinforcing? }\end{array}$ & No & $\begin{array}{l}\text { Only live video feed } \\
\text { available, no } \\
\text { dimensioning software. }\end{array}$ \\
\hline $\begin{array}{l}\text { Can the system be } \\
\text { used to monitor the } \\
\text { vibration process? }\end{array}$ & Yes & $\begin{array}{l}\text { Live video feed shows } \\
\text { the action being } \\
\text { completed. }\end{array}$ \\
\hline $\begin{array}{l}\text { Can the system be } \\
\text { used to determine } \\
\text { whether the vibration } \\
\text { is adequate and that } \\
\text { the correct compaction } \\
\text { is obtained? }\end{array}$ & No & $\begin{array}{l}\text { Difficult to know } \\
\text { exactly when vibration } \\
\text { is adequate. }\end{array}$ \\
\hline $\begin{array}{l}\text { Can the system be } \\
\text { used to monitor the } \\
\text { measures taken to } \\
\text { prevent grout loss? }\end{array}$ & Yes & $\begin{array}{l}\text { Live video feed makes } \\
\text { it possible. }\end{array}$ \\
$\begin{array}{l}\text { Can the system be } \\
\text { used to check whether } \\
\text { cover blocks have been } \\
\text { inserted? }\end{array}$ & Yes & $\begin{array}{l}\text { Live video feed shows } \\
\text { the completion of the } \\
\text { action. }\end{array}$ \\
\hline $\begin{array}{l}\text { Can the system be } \\
\text { used to measure the } \\
\text { dimensions of the cover } \\
\text { blocks? }\end{array}$ & No & $\begin{array}{l}\text { Only live video feed } \\
\text { available, no } \\
\text { dimensioning software. }\end{array}$ \\
\hline
\end{tabular}




\begin{tabular}{|c|c|c|}
\hline $\begin{array}{l}\text { Can the system be } \\
\text { used to check whether } \\
\text { actions have been } \\
\text { engaged in to prevent } \\
\text { the kicking of } \\
\text { formwork? }\end{array}$ & Yes & $\begin{array}{l}\text { Live video feed shows } \\
\text { the action being } \\
\text { completed. }\end{array}$ \\
\hline $\begin{array}{l}\text { Can the system be } \\
\text { used to check whether } \\
\text { bleeding occurs after } \\
\text { power floating the } \\
\text { concrete? }\end{array}$ & Yes & $\begin{array}{l}\text { Live video feed shows } \\
\text { the presence of } \\
\text { bleeding. }\end{array}$ \\
\hline $\begin{array}{l}\text { Can the system be } \\
\text { used to measure the } \\
\text { slump and check the } \\
\text { consistency of the } \\
\text { concrete mix? }\end{array}$ & No & $\begin{array}{l}\text { Live video feed shows } \\
\text { the action being } \\
\text { completed, but cannot } \\
\text { measure the slump. }\end{array}$ \\
\hline $\begin{array}{l}\text { Can the technology be } \\
\text { used to inspect the } \\
\text { cleanliness of the } \\
\text { equipment or to } \\
\text { monitor the cleaning of } \\
\text { equipment on site? }\end{array}$ & Yes & $\begin{array}{l}\text { Live video feed shows } \\
\text { the action being } \\
\text { completed. }\end{array}$ \\
\hline $\begin{array}{l}\text { Can the system be } \\
\text { used to determine } \\
\text { when the curing } \\
\text { process is adequate to } \\
\text { ensure a durable } \\
\text { concrete? }\end{array}$ & No & $\begin{array}{l}\text { System cannot provide } \\
\text { information regarding } \\
\text { relative humidity and } \\
\text { moisture conditions. }\end{array}$ \\
\hline $\begin{array}{l}\text { Can the system be } \\
\text { used to monitor the } \\
\text { process of applying } \\
\text { curing principles? }\end{array}$ & Yes & $\begin{array}{l}\text { Live video feed shows } \\
\text { the action being } \\
\text { completed. }\end{array}$ \\
\hline $\begin{array}{l}\text { Can the system be } \\
\text { used to determine the } \\
\text { lap-lengths of } \\
\text { reinforcing? }\end{array}$ & No & $\begin{array}{l}\text { Only live video feed } \\
\text { available, no } \\
\text { dimensioning software. }\end{array}$ \\
\hline $\begin{array}{l}\text { Can the system be } \\
\text { used to check whether } \\
\text { the pull-out bars are } \\
\text { correct? }\end{array}$ & No & $\begin{array}{l}\text { Cannot determine level } \\
\text { of pull-out bars. }\end{array}$ \\
\hline $\begin{array}{l}\text { Does the technological } \\
\text { option provide footage } \\
\text { to identify surface } \\
\text { deformations on } \\
\text { shutter boards? }\end{array}$ & Yes & $\begin{array}{l}\text { Live video feed enables } \\
\text { the user to see } \\
\text { formwork condition. }\end{array}$ \\
\hline $\begin{array}{l}\text { Can the system be } \\
\text { used to ensure that the } \\
\text { equipment and } \\
\text { machinery is } \\
\text { adequately cleaned } \\
\text { before use? }\end{array}$ & Yes & $\begin{array}{l}\text { Live video feed shows } \\
\text { the action being } \\
\text { completed. }\end{array}$ \\
\hline
\end{tabular}




\begin{tabular}{l|l|l}
\hline $\begin{array}{l}\text { Can the system be } \\
\text { used to determine the } \\
\text { correct location of the } \\
\begin{array}{l}\text { Holding down (HD) } \\
\text { bolts? }\end{array}\end{array}$ & No & $\begin{array}{l}\text { Live video feed shows } \\
\text { the action being } \\
\text { completed. }\end{array}$ \\
\hline $\begin{array}{l}\text { Can the system be } \\
\text { used for material } \\
\text { handling on-site? }\end{array}$ & No & $\begin{array}{l}\text { Provides no } \\
\text { information regarding } \\
\text { material. }\end{array}$ \\
\hline $\begin{array}{l}\text { Can the system be } \\
\text { used to check the } \\
\text { dimensions and } \\
\text { alignment of } \\
\text { formwork? }\end{array}$ & No & $\begin{array}{l}\text { Require BIM and } \\
\text { dimensioning software. }\end{array}$ \\
\hline
\end{tabular}

\section{Probability density functions}

An approach, to determine the effectiveness of the laser scanner and IDS for variable accuracy requirements, were developed. This approach is additional to the study and is only used in the event that the user desires a different accuracy in the rating criteria. This approach is only applicable where the laser scanner and IDS are used for dimensioning. This approach is limited in the manner that it only considers variable accuracy requirements and not variations in duration to monitor checks.

\section{C.1 Approach for the laser scanner}

A probability density function (PDF) was developed for a range of accuracy deviation percentages. A probability density function is used to describe a continuous probability distribution. Goodness-of-fit tests were conducted to determine the correct density function that relates to the data. A goodness-of-fit test is used to determine the best population fit of a data sample.

The Kolmogorov-Smirnov (K-S) and the Anderson-Darling (A-D) tests were used to determine a distribution that best fit the data. A significance level of $\alpha=0.05$ was chosen for the test, since that is the most common value used for the K-S test. A significance level of 0.05 indicates a $5 \%$ risk of concluding that a difference exists when there is no actual difference. In the hypothesis test it would be required that a $\mathrm{P}$-value greater than the significance level $(\alpha=0.05)$ be obtained to accept the null hypothesis (Minitab Inc. 2015).

The test was conducted as follows:

$H_{0}=$ The data follows a normal distribution

$H_{1}=$ The data does not follow a normal distribution

Significance level $(\alpha)=0.05$

The raw data, consisting of accuracy deviation percentages, were imported into statistical software to compute the goodness-of-fit to a normal distribution. The sample size was $n=44$, after removing the outliers.

The p-values for the K-S and A-D tests were computed in the software and are shown in Table 4 .

Table 4: P-values obtained for the K-S and A-D goodness-of-fit tests.

\begin{tabular}{ll}
\hline Test & P-value \\
\hline Kolmogorov-Smirnov & 0.796 \\
\hline Anderson-Darling & 0.707 \\
\hline
\end{tabular}




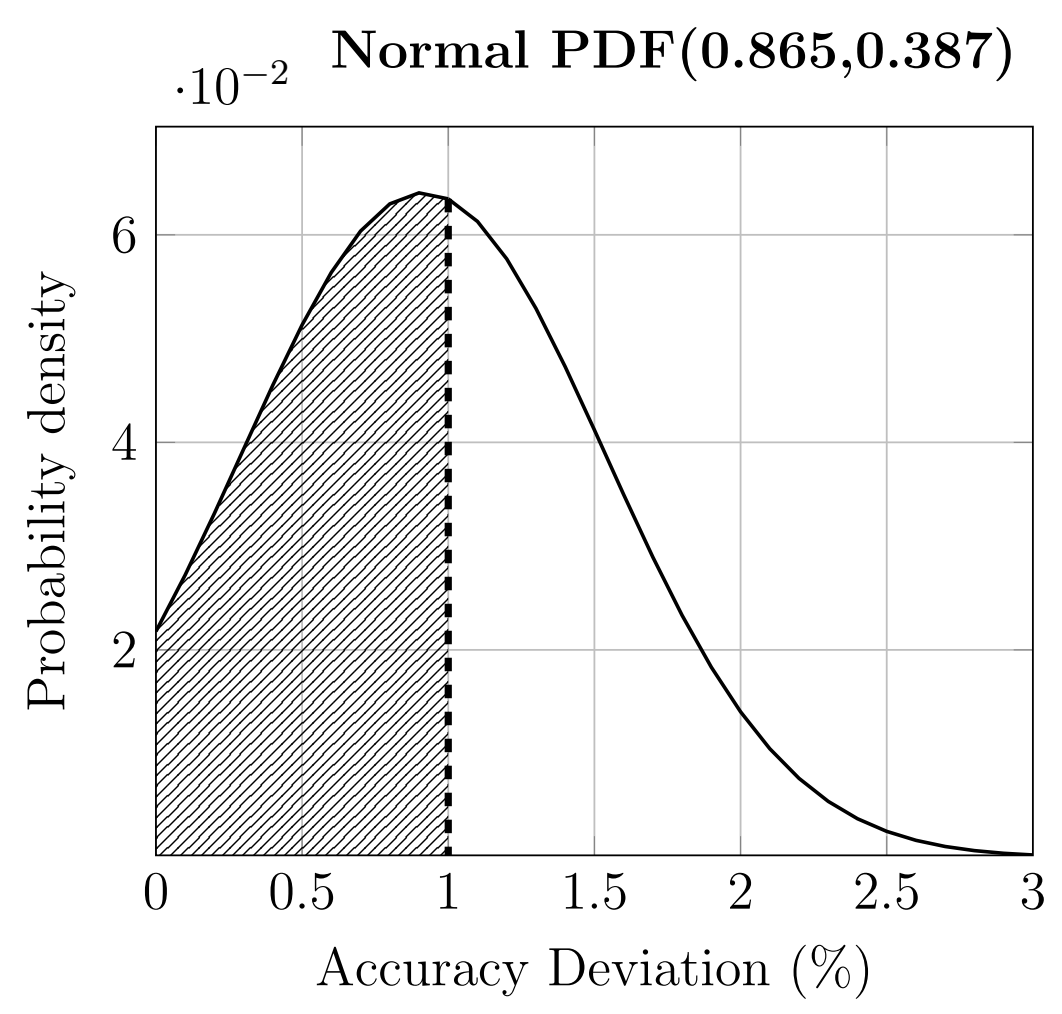

Figure 3: PDF, with mean of $0.865 \%$ and variance of 0.387 , for laser scanner software monitor steel spacing

The PDF in Figure 3 is used by choosing an accepted accuracy deviation that is required from the technology and then calculating the corresponding probability of success. As an example, the user requires an accuracy deviation which is less than $1 \%$ for the technology to be successful. Calculating the probability of success then results in a $58 \%$ effectiveness. For a different project the user might require an accuracy deviation which is less than $1.5 \%$ and then the technology has an estimated effectiveness of $83 \%$.

In both cases the P-value is greater than the significance level of 0.05 , therefore we fail to reject the null hypotheses. The probability distribution is assumed to be normal. Figure 3 shows the normal distribution determined from the data. The data is represented by a normal distribution with a mean deviation percentage of $0.865 \%$ and standard deviation of $0.662 \%$.

The effectiveness result from the PDF method is compared that of the histogram method. The effectiveness is calculated by determining the probability $\mathrm{P} \leq 1 \%$ since that corresponds to a rating of $\geq 4$ out of 5 as specified in Table 5.3 . The effectiveness, from the PDF method, was calculated as $58 \%$. This corresponds very closely to the effectiveness $(57 \%)$ determined through the histogram method.

The PDF method was only based on the accuracy deviation of the laser scanner. The time allowed for each rating criteria will influence the ultimate effectiveness of the technology. As a simplification, only the accuracy was considered as a variable that influence the effectiveness of the technology.

\section{C.2 Approach for IDS effectiveness}

A probability density function (PDF) was developed to determine the effectiveness of the technology for various accuracy requirements. The required accuracy of the technology can vary depending on the use of the technology. The accuracy deviation percentages, obtained through laboratory tests, were used to develop the PDF. The user can determine the expected effectiveness of the technology by calculating the probability of the technology obtaining the desired accuracy. 
The goodness-of-fit test was conducted as follows:

$H_{0}=$ The data follows a log-normal distribution

$H_{1}=$ The data does not follow a log-normal distribution

Significance level $(\alpha)=0.05$

The ratings, shown in Table 5.5, were imported into statistical software to compute the goodness-of-fit to a log-normal distribution. The sample size was $n=13$, after removing an extreme outlier. For a more accurate estimation, a larger sample size is required.

The p-values for the K-S and A-D tests were computed in the software and are shown in Table 5 ;

Table 5: P-values obtained for the K-S and A-D goodness-of-fit tests.

\begin{tabular}{ll}
\hline Test & P-value \\
\hline Kolmogorov-Smirnov & 0.773 \\
\hline Anderson-Darling & 0.878 \\
\hline
\end{tabular}

In both cases the P-value is greater than the significance level of 0.05 , therefore we fail to reject the null hypothesis. The data sample is assumed to be from a log-normal distribution.

The log-normal distribution, in Figure 4, represents the expected probability for obtaining a required accuracy deviation percentage. The data is represented by a normal distribution with a mean deviation of $0.923 \%$, standard deviation of $1.102 \%$ and skewness of 2.024 .

The technology effectiveness obtained through the histogram method and the PDF method was compared. The effectiveness of the technology, through the PDF method, is calculated by determining the probability $\mathrm{P}(\mathrm{x} \leq 1)$, since that corresponds to a rating of $\geq 4$ out of 5 as specified in Table 5.3 The effectiveness was calculated as $71.5 \%$. This corresponds very closely to the effectiveness $(72 \%)$ determined through the histogram method. 


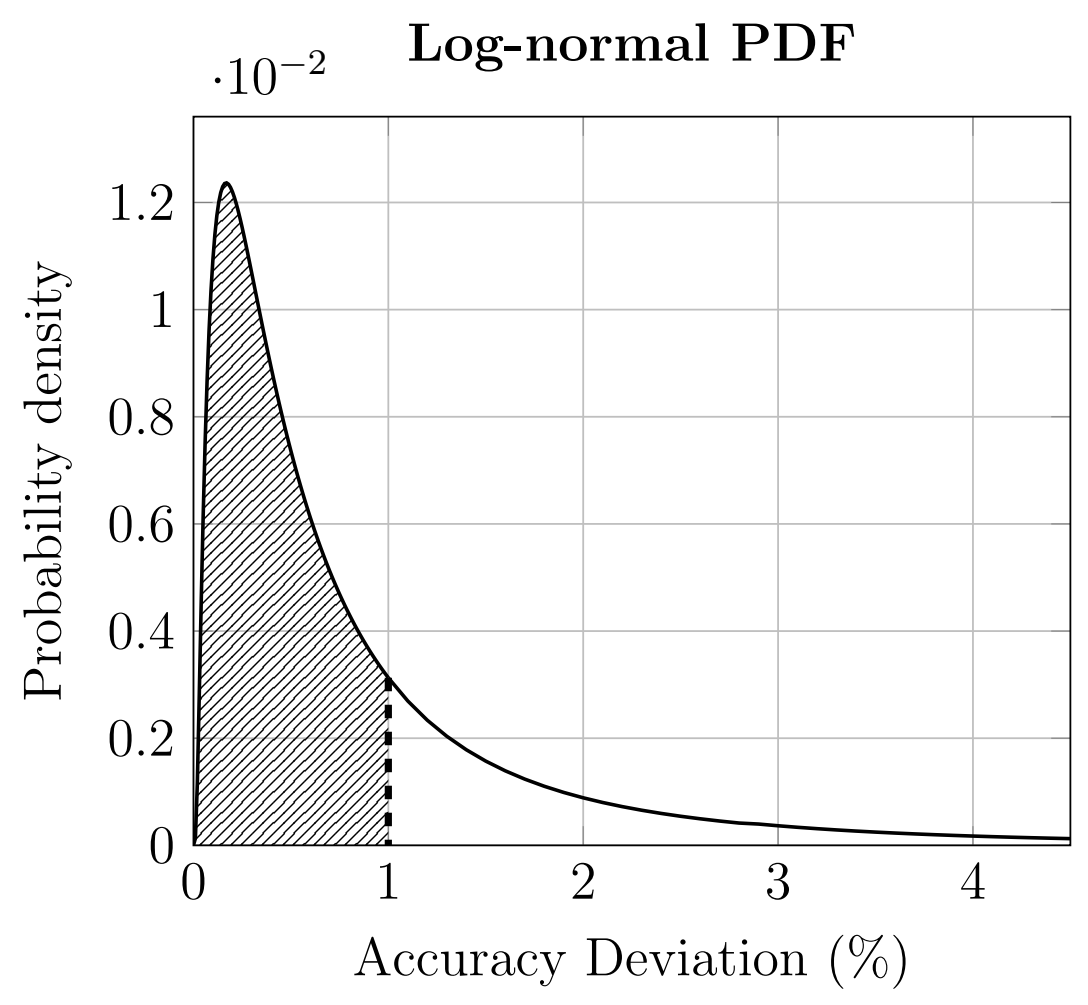

Figure 4: Log-normal PDF with mean of $0.923 \%$ and variance of 1.214 , for laser scanner software monitor steel spacing

\section{Technological specifications and cost}

In this Section, various technological solutions are investigated to monitor construction processes. The investigated technologies include the following:

- Laser scanning

- Photography and video monitoring

- Software for daily reporting and logging

- Image dimensioning software

- Radio frequency identification

- Quick response codes

- Concrete sensors

The advantages and disadvantages of the systems were investigated together with the cost to implement such systems. Other technical specifications of the technologies were determined as well.

\section{D.1 Laser scanning technology}

Three types of systems for laser scanning were identified and investigated. System 1 can be used during walking, flying or driving. System 2 is a handheld scanner and is more applicable to use when scanning objects with low volume. System 3 is used in the form of a tripod scanner and scans the objects around it. 


\section{D.1.1 System 1: Walk, Drive and Fly}

A laser scanner captures data across a variety of applications. It is useful in collecting data when walking, driving and flying. The system hardware specifications are as follows (3D Laser mapping, 2016):

- High accuracy laser scanner (3mm-5mm)

- $12 \mathrm{MP}$ camera for driving

- $18.4 \mathrm{MP}$ camera for walking and flying

- GIS and inertial measurement unit (IMU)

- Touch screen control unit

- 3 mounting systems

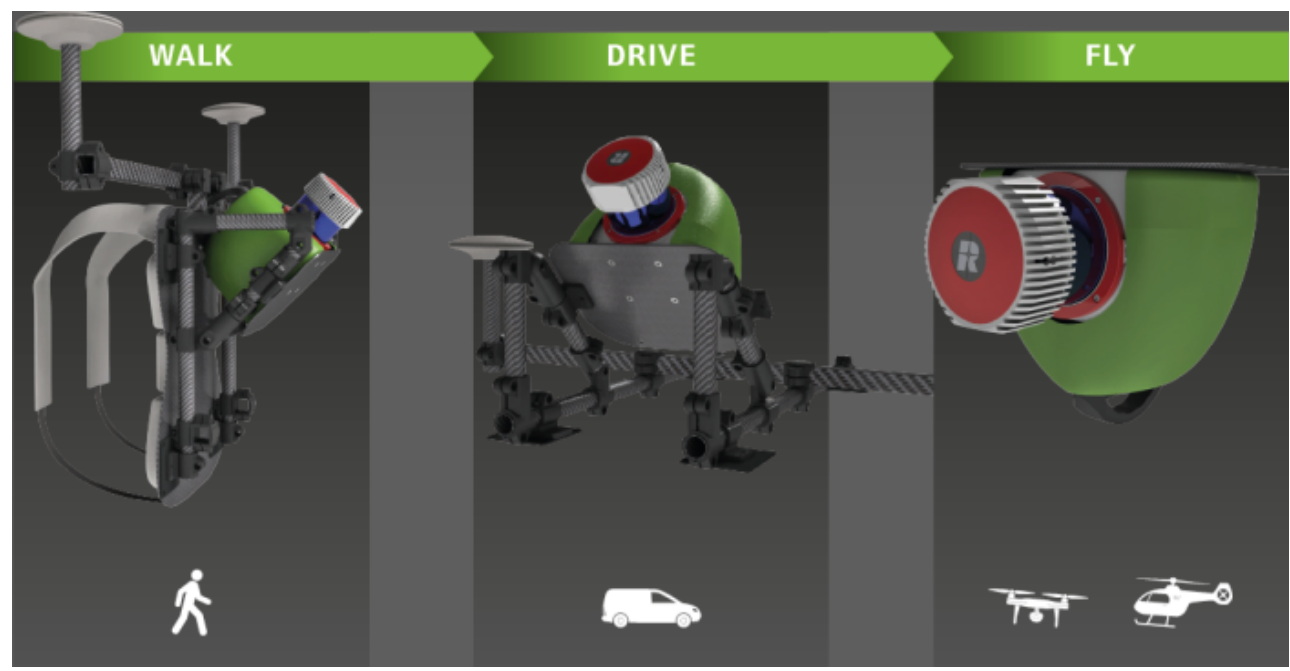

Figure 5: System 1: Can be used to generate scans while walking, flying and driving (3D Laser mapping, 2016)

The system includes the software necessary for the capturing and post-processing of data. When purchasing the system, it includes 1-year software maintenance and support together with a 1-year hardware warranty and support. Training to assist in the operation of the technology is also included in the cost of the system. The system provides a 330-degree field of view with a maximum scan range of $920 \mathrm{~m}$. The maximum effective measurement rate of the scanner is 1 million points per second (3D Laser mapping, 2016).

The advantage of the system is that it contains an IMU that ensures accurate measurements during walking, driving and flying. After conversations with the Regional General Manager of 3D Laser Mapping it was determined that the scanners are capable of scanning dark objects, which have previously been a concern, and that various projects have been completed successfully where the scanning of black coal faces have been conducted. The cost of the system, which includes the software and training, was estimated at R 4000000 (four million Rand).

\section{D.1.2 System 2: Handheld scanner}

The handheld laser scanning system, as indicated in Figure 6, can capture almost any surface type in a wide range of environments. The system provides real-time point cloud visualisation for intuitive data acquisition. The device includes a tablet with the required software loaded on it. The system is easy to use and does not require an extra power supply during usage. It has a range of 0.5 to $3 \mathrm{~m}$ with a $3 \mathrm{D}$ point accuracy of less than $1.5 \mathrm{~mm}$. The device records 88,000 points per second. The scan volume is limited to $8.1 \mathrm{~m}^{3}$. The typical field of view at a $1 \mathrm{~m}$ distance is $930 \mathrm{~mm} \times 1,100 \mathrm{~mm}$ and increases further away from 
the object. The angular field of view is $45 \mathrm{x} 59$ degrees (height $\mathrm{x}$ width). The identified scanner weighs only $0.98 \mathrm{~kg}$ (Faro, 2016). The price of the device is estimated at R320,000 (excluding VAT). The training required to operate the device was estimated at R8000/day plus the travelling and accommodation cost for the trainers.

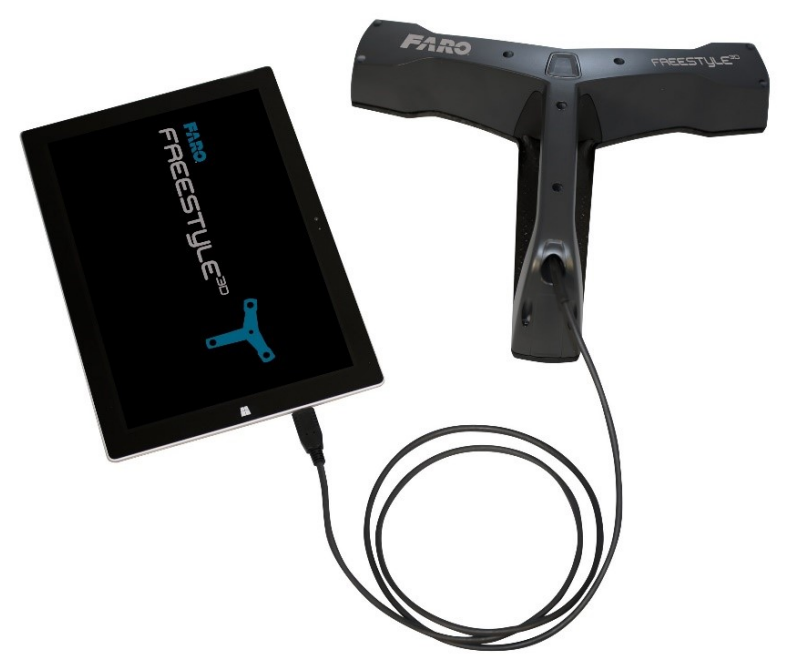

Figure 6: System 2: Handheld scanner that shows scanned objects while scanning (Faro, 2016)

\section{D.1.3 System 3: Scanner on a tripod}

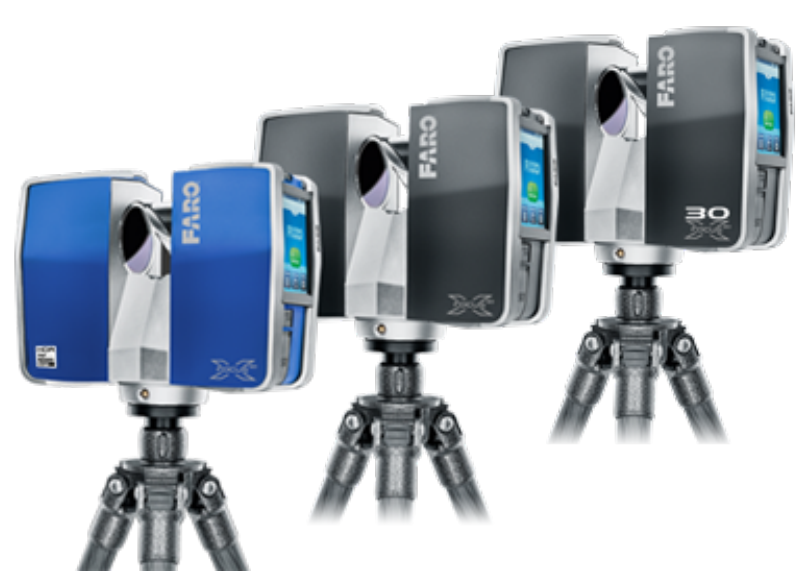

Figure 7: System 3: Tripod scanner (Faro, 2016)

The laser scanner of System 3 is a mid-range laser scanner with a scanning range of up to $130 \mathrm{~m}$. It is integrated with a GPS receiver to determine the position of the scanner. This aids to facilitate the registration process and to provide the exact time and location of the user's scans. The scanner has a battery run-time of 4.5 hours and makes use of a SD card to store the data. The scanner data is saved on the SD card, afterwards the SD card is placed in a PC and the data is processed by the software. The scanner scans up to 1-million points per second. The noise of the scanner is very low at $0.15 \mathrm{~mm}$ and 
scans with an accuracy of $+/-2 \mathrm{~mm}$. The scanner has a view of 300 degrees vertically and 360 degrees horizontally.

The cost of the scanner is R515,000 (excluding VAT). Additionally, it is required to purchase the software at R220,000 together with two boxes of the alignment spheres at R38,000 a box. It is also required to purchase the tripod at R9,000. At least 3 days of training is required to operate the scanner. The training costs are R8000/day plus the required travelling and accommodation costs of the training team.

The HDR (High Dynamic Range) system of the same type of scanner has High definition (HD) photo resolution which provides better photo quality. The cost of the HDR device is R780,000. Another device of the same type has an extended range of $330 \mathrm{~m}$. The cost for the scanner is $\mathrm{R} 650,000$. The additional costs for the training, tripod and spheres also need to be added.

The price for the HD scanner with $330 \mathrm{~m}$ range is R940,000 plus the additional costs as mentioned. The other option provided is to make use of the scanning services. The rates for scanning are estimated at R18,000 per day plus the travel and accommodation costs of the scanning team (iQLaser, 2016).

\section{D.1.4 Assessing the various laser scanner systems}

The capability of the various laser-scanning systems was assessed from the information presented in Chapter D. Chapter D shows the technical specifications of the various systems. It is important to ensure that the accuracy of a system is within the tolerance limits as specified by SANS 2001-CC1 (2007). The SANS 2001-CC1 (2007) applies to concrete works designed in accordance with the requirements of SANS 10100-1.

The three laser scanning systems under consideration are summarised as follows:

- System 1: Walk, drive and fly

- System 2: Handheld scanner

- System 3: Scanner on tri-pod

Table 4.2 shows the accuracy of the various laser scanners when compared to the permissible deviations for the required degrees of accuracy as specified by SANS 2001-CC1 (2007). A technological system used for dimensioning has to provide readings that are more accurate than the permissible deviations in Table 4.2. In Table 4.2, various quality checks have been identified together with the permissible deviation of each. Systems 1 to 3 provide data that are within the acceptable accuracies, therefore a system would be chosen based on the type of situation in which it is required. System 1 is more applicable to use where large volume scans are required and where access to the work is difficult or dangerous. System 2 , the handheld scanner, is more applicable to use where the scanning of single elements, with a volume of less than $8 \mathrm{~m}^{3}$, is required. System 3, the tripod scanner, is more applicable to use when scanning large volumes or single elements. From Table 4.2 on p 65, it is evident that the accuracy of the laser scanners enable it to be used for the three Degrees of Accuracy as specified in SANS 2001-CC1 (2007).

\section{D.2 Summary of the cost for the laser scanner systems}

Table 6 shows the total estimated cost for the various laser scanner systems. 
Table 6: Summary of the costs for the various systems identified

\begin{tabular}{|c|c|c|c|c|}
\hline $\begin{array}{l}\text { Laser scanner } \\
\text { system }\end{array}$ & Technical & Attributes & & Cost \\
\hline System 1 & $\begin{array}{c}\text { Accuracy } \\
\text { Range } \\
\text { Points/second } \\
\text { Setup }\end{array}$ & $\begin{array}{c}3 \text { to } 5 \mathrm{~mm} \\
920 \mathrm{~m} \\
\text { 1-million } \\
\text { Walk/drive/fly }\end{array}$ & $\begin{array}{c}\text { Device } \\
\text { Training } \\
\text { Software } \\
\text { Extras } \\
\text { Total }\end{array}$ & $\begin{array}{cc} & \mathrm{n} / \mathrm{a} \\
\mathrm{n} / \mathrm{a} \\
\mathrm{n} / \mathrm{a} \\
\mathrm{n} / \mathrm{a} \\
\mathbf{R} 4 \text { million }\end{array}$ \\
\hline $\begin{array}{l}\text { System 2 } \\
\left(8 m^{3} \text { limit }\right)\end{array}$ & $\begin{array}{c}\text { Accuracy } \\
\text { Range } \\
\text { Points/second } \\
\text { Setup }\end{array}$ & $\begin{array}{c}1.5 \mathrm{~mm} \\
920 \mathrm{~m} \\
\text { 1-million } \\
\text { Hand-held }\end{array}$ & $\begin{array}{c}\text { Device } \\
\text { Training } \\
\text { Software } \\
\text { Extras } \\
\text { Total }\end{array}$ & $\begin{array}{c}\mathrm{R} 320000 \\
\mathrm{R} 16000 \\
\mathrm{n} / \mathrm{a} \\
\mathrm{n} / \mathrm{a} \\
\text { R } \mathbf{3 3 6 \mathbf { 0 0 0 }}\end{array}$ \\
\hline $\begin{array}{l}\text { System } 3.1 \\
(130 \mathrm{~m})\end{array}$ & $\begin{array}{c}\text { Accuracy } \\
\text { Range } \\
\text { Points/second } \\
\text { Setup }\end{array}$ & $\begin{array}{c}2 \mathrm{~mm} \\
130 \mathrm{~m} \\
\text { 1-million } \\
\text { Tripod }\end{array}$ & $\begin{array}{c}\text { Device } \\
\text { Training } \\
\text { Software } \\
\text { Extras } \\
\text { Total }\end{array}$ & $\begin{array}{c}\text { R } 515000 \\
\text { R } 24000 \\
\text { R } 220000 \\
\text { R } 76000 \\
\text { R } 835 \mathbf{0 0 0}\end{array}$ \\
\hline $\begin{array}{l}\text { System 3.2 } \\
(\text { HD 130m) }\end{array}$ & $\begin{array}{c}\text { Accuracy } \\
\text { Range } \\
\text { Points/second } \\
\text { Setup }\end{array}$ & $\begin{array}{c}2 \mathrm{~mm} \\
130 \mathrm{~m} \\
\text { 1-million } \\
\text { Tripod }\end{array}$ & $\begin{array}{c}\text { Device } \\
\text { Training } \\
\text { Software } \\
\text { Extras } \\
\text { Total }\end{array}$ & $\begin{array}{c}\text { R } 780000 \\
\text { R } 24000 \\
\text { R } 220000 \\
\text { R } 76000 \\
\text { R } 1.1 \text { million }\end{array}$ \\
\hline $\begin{array}{l}\text { System 3.3 } \\
(330 \mathrm{~m})\end{array}$ & $\begin{array}{c}\text { Accuracy } \\
\text { Range } \\
\text { Points/second } \\
\text { Setup }\end{array}$ & $\begin{array}{c}2 \mathrm{~mm} \\
330 \mathrm{~m} \\
\text { 1-million } \\
\text { Tripod }\end{array}$ & $\begin{array}{c}\text { Device } \\
\text { Training } \\
\text { Software } \\
\text { Extras } \\
\text { Total }\end{array}$ & $\begin{array}{c}\text { R } 650000 \\
\text { R } 24000 \\
\text { R } 220000 \\
\text { R } 76000 \\
\text { R 970 000 }\end{array}$ \\
\hline $\begin{array}{l}\text { System 3.4 } \\
(\mathrm{HD} 330 \mathrm{~m})\end{array}$ & $\begin{array}{c}\text { Accuracy } \\
\text { Range } \\
\text { Points/second } \\
\text { Setup }\end{array}$ & $\begin{array}{c}2 \mathrm{~mm} \\
330 \mathrm{~m} \\
\text { 1-million } \\
\text { Tripod }\end{array}$ & $\begin{array}{c}\text { Device } \\
\text { Training } \\
\text { Software } \\
\text { Extras } \\
\text { Total }\end{array}$ & $\begin{array}{c}\text { R } 940000 \\
\text { R } 24000 \\
\text { R } 220000 \\
\text { R } 76000 \\
\text { R } 1.26 \text { million }\end{array}$ \\
\hline
\end{tabular}

It is seen that the System 2, the hand-held scanner, is the most cost effective. The only disadvantage is that the system can only scan $8.1 \mathrm{~m}^{3}$ at a time. The investor should consider the nature of the scans required before purchasing such a scanner. System 3.1 is the next most cost effective. This is the preferred system since it has a long scan range, good accuracy and and permits large scan volumes.

\section{E Photography and video monitoring}

For photography and video monitoring, the use of the following technologies are considered:

- Photo-log daily reporting

- Image dimensioning software

- IP cameras 


\section{E.1 Software application for photo-log daily reporting}

The cost for such an application was estimated at $\$ 37.00(\mathrm{R} 550.00)$ per month. The cost of a mobile phone/tablet with a good camera needs to be included as well. The cost of a phone/tablet was estimated at R10, 000.00. At least two mobile devices are required. The total cost of the software was estimated for a one-year subscription (Raken, 2016). The total cost of the system for one year is equal to R26, 600.00 .

\section{E.2 Mobile software that is used to measure dimensions on im- ages}

The total cost of the system would include only the use of hardware. The hardware includes at least two mobile phones or tablet devices that has a good camera. The cost of such a system was estimated at R20, 000.00 (Price Check, 2017).

\section{E.3 Internet Protocol Cameras}

The prices of various cameras were analysed and the specifications of a camera that meets minimum requirements of quality are shown in Table 7. Such a camera cost +/- R3, 000 (Price Check, 2017).

Table 7: Technical specifications of a proposed IP camera

\begin{tabular}{ll}
\hline \multicolumn{2}{c}{ Technical specification } \\
\hline Resolution & $1080 \mathrm{p}$ \\
Frames per second & $30 \mathrm{fps}$ \\
Field of view & 360 degrees \\
Digital zoom & $8 \mathrm{x}$ \\
Motion detection & Yes \\
Two-way audio & Yes \\
\hline
\end{tabular}

Prices were obtained for the other hardware components of the system as well. These prices are as follows (Price Check, 2017):

- Router cost: R500.00

- Uninterrupted Power Supply (UPS): R1,600.00

- Gigabit switch: R1,500.00

- NVR: R1,500.00

For a system consisting of four cameras the total cost (excluding the PC running the NVR) would be more or less R17, 100.00. Including the cost of a PC, the system cost was estimated at R30, 000.00. It was reasoned that at least four cameras are required to monitor a site adequately. 


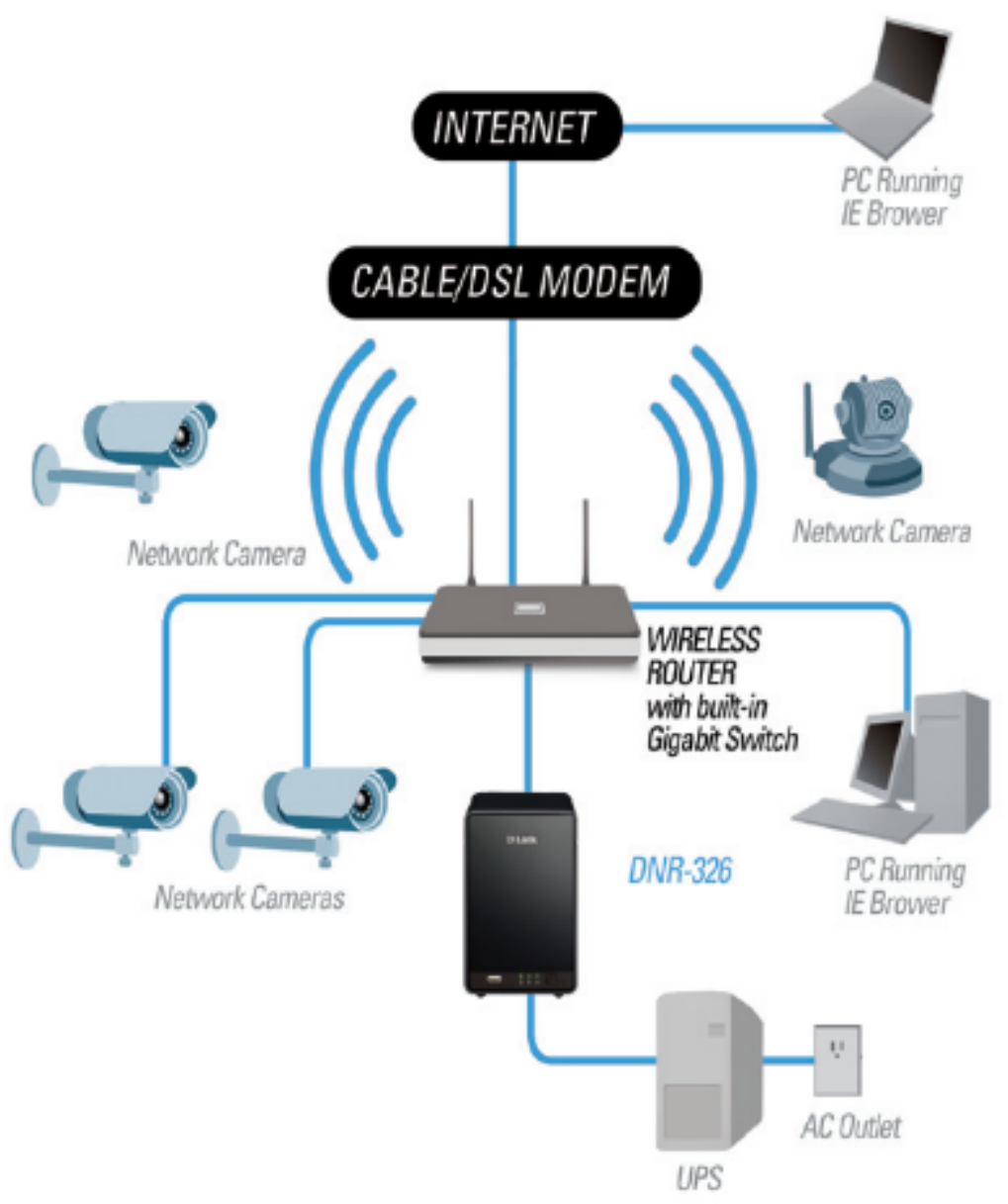

Figure 8: IP camera network setup

Figure 8 shows the setup for a typical IP camera system. Cameras are connected to the network by means of Ethernet or Wi-Fi. There is also a network video recorder, indicated as DNR-326, which allows for concurrent recording and viewing either in the premises or remotely via the internet. The Uninterrupted Power Supply (UPS) ensures that the recording continues during a loss in power supply. The wireless router in Figure 8 has a gigabit switch which allows the sending of power to each camera through the network cable (Emailitis, 2012).

\section{F Radio Frequency Identification tags (RFID)}

Reader antennas can operate in either a short range or a long range. The short range distance between a reader and a tag is less than $30 \mathrm{~cm}$. The advantage of short range readers is that they are not influenced by the presence of dielectrics such as water and metals. Far range readers can operate between $30 \mathrm{~cm}$ and tens of meters. The reader software is used to send commands to the reader to help connect the RFID readers with certain tag information (Impinj, 2016).

The hardware required to install such a system is the reader, tag chips, tag antennas, reader antennas and reader control software. The low frequency systems have a shorter read range, but has the ability to operate near metal and liquid surfaces. The higher frequency systems have longer read ranges, but are more sensitive radio interference caused by metals and liquids (Impinj, 2016).

The interaction of the system components required for the operation of a RFID system is shown in Figure 9. The system components required are as follows (Ropraz, 2008): 
- Tags

- Reader/Writer: Also known as transceivers

- Two or more antennas (One for the tag and one for the reader)

- Application software (middle-ware) and a host computer system.

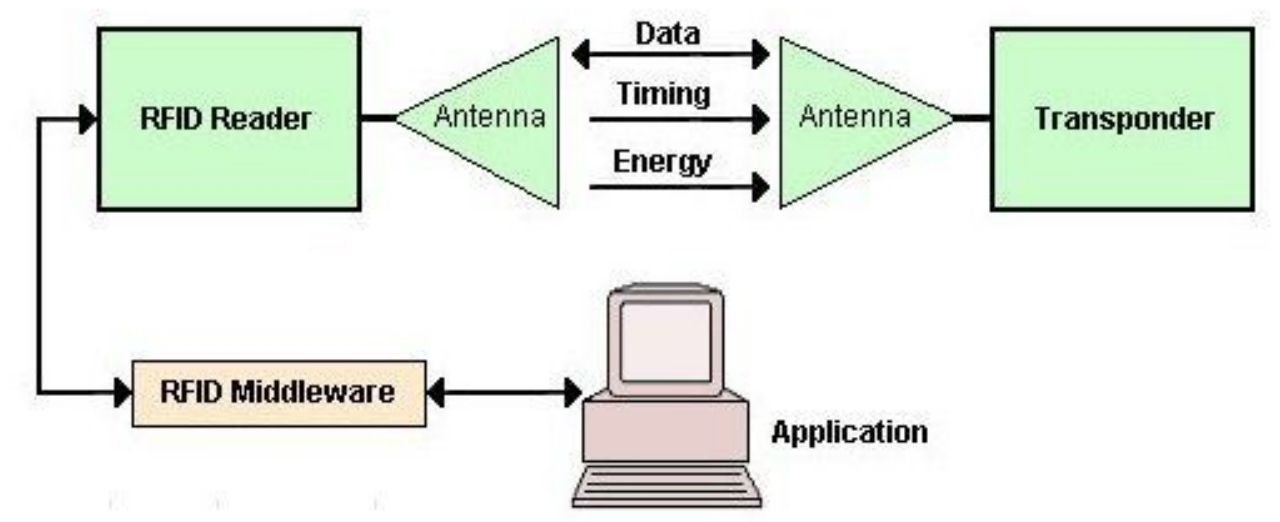

Figure 9: RFID system (Ropraz, 2008)

From a study by Jaselskis, Anderson, Jahren, Rodriguez and Njos (1995) it was found that the RFID tags were advantageous to use for the processing of concrete, handling of equipment and materials control. Jaselskis et al. (1995) identified that the RFID tags were useful to ensure efficient material handling when supplies are delivered at a single location on site. A study by Wang et al. (2015) in Taiwan also showed that a RFID system in a concrete specimen laboratory made the concrete testing process more efficient. It was more efficient, since it was not necessary for the laboratory staff to complete loads of paper work. It also reduced the time and space gap between the laboratory and project participants due to the records being updated on a central server.

\section{F.1 Technical requirements and cost of system}

A RFID system kit was identified and it consisted of a fixed reader and 100 tag transponders. The reader is connected directly to a computer to display the received codes. The reader system operates on a power supply of $110-120$ volts or 220-240 volts. The cost of the entire system was identified as R38, 850.00 (Price Check, 2017).

\section{F.2 Quick response (QR) codes}

The hardware required to operate such a system is a computer and a mobile phone. The disadvantage identified with such a system is that the administration behind the operation of the system could be time-consuming. The estimated cost of such a system is as follows (Price Check, 2017):

- Computer $=$ R10 000.00

- Mobile phone = R6 000.00

A QR code system can be used in a similar manner as the RFID system. The advantage of the QR code is that the hardware is lower in cost. 


\section{G Concrete curing sensors}

The sensors have the following features:

- Temperature sensor accuracy $=+/-0.40$ degrees Celsius

- $\mathrm{RH}$ sensor accurate within $+/-3$

- The sensor can be embedded up to $15 \mathrm{~cm}$ from surface of concrete

- Sensors are installed with a zip tie

- Data is interpretable via software on a smartphone

- The casing of the sensor is resistant to chemicals and has high strength

- Temperature range between -30 to 85 degrees Celsius

Other systems exist which are not wireless. The sensors of these systems are embedded in the concrete with a cable protruding from the surface of the concrete. The cable is then connected to a hand-held device which displays the information. Such a system costs $\$ 3,000.00$ (R 45, 000.00) (The Transtec Group, 2015). The device is shown in Figure 10.

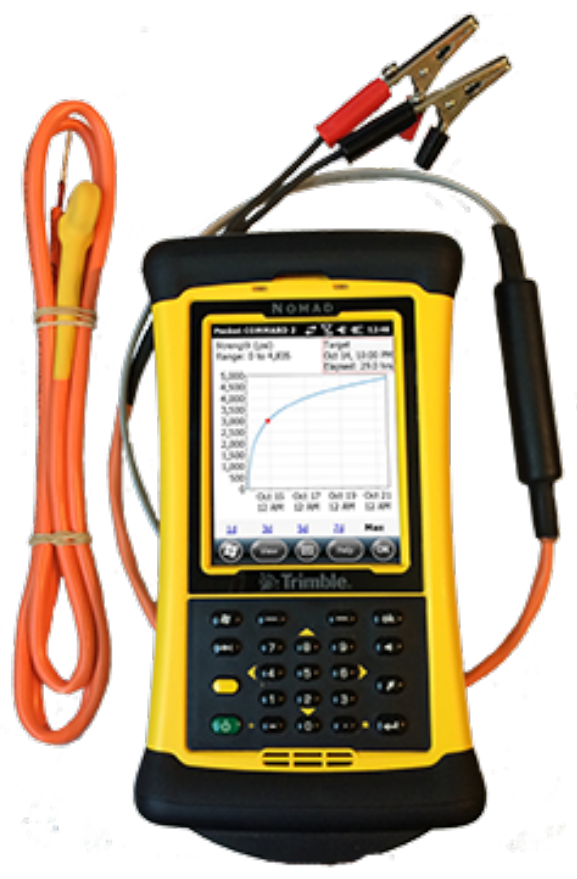

Figure 10: Concrete sensors that work with probes (The Transtec Group, 2015)

\section{H Summary of cost for the various systems}

The cost of the laser systems are available in Table 6 . Table 8 shows the estimated costs to implement the other technological systems. The cost of the hardware were determined for what the minimum requirements would be on a construction site. 
Table 8: Summary of the cost for the various technological systems identified

\begin{tabular}{lll}
\hline System & Cost (Rand) & Quantity \\
\hline Photo-log software & R 26 600.00 & 2x mobile devices + software (1-year subscription) \\
Dimensioning software & R 20000.00 & 2x mobile devices + software (1-year subscription) \\
IP Camera system & R 30000.00 & 4x cameras + other hardware \\
RFID System & R 38 850.00 & 100x tags + 1x reader + 1x pc \\
QR Code & R 16000.00 & 1x computer + 1x mobile phone \\
Curing sensor system & R 45000.00 & 1x system kit as proposed by The Transtec Group (2015) \\
\hline
\end{tabular}

From Table 8 it was shown that most of the systems require low implementation costs. The use of laser scanners is more expensive than the other systems. All the other systems, besides laser scanning, were relatively low in cost.

\section{Survey}




\section{Quality control of structural concrete}

1. Participant information

Years experience in

Contracting

Years experience in

Consulting

ECSA registration

number (Optional)

Email Address (Only for

CPD candidates)

\section{Introduction to CPD activity}

The name and number of the participant will be kept confidential and the individual response to the survey will not be shared with any party. The participant has the right to withdraw from the survey at any time, and is not obliged to either participate or complete the survey.

Participants in this survey can earn 0.1 CPD points from ECSA by completing this questionnaire (1) and a follow up feedback questionnaire (2). This is in line with the ECSA requirement that 1 CPD point can be obtained for 10 hours of activity participation.

The points can only be allocated upon completion of both questionnaires.

For purpose of CPD records, the ECSA registration number of the participant is requested.

CPD candidates have to read and complete the entire survey. Non-CPD point candidates only have to complete the survey sections, which are indicated in blue.

\section{CPD READING}

\section{Abstract}

The study under investigation aims to set up a framework that proposes various technological solutions to monitor quality aspects during the construction of structural concrete works. The framework is derived from user input data that specifies the expected performance of labour, management, subcontractors and equipment on a specific project. Each of the quality aspects are seen as a risk that could lead to rework on the project when not monitored adequately. The purpose of the technological solutions are to serve as a mitigation technique for the risks identified. 


\section{Content}

The Business Dictionary (2013)defines Quality as:

"A measure of excellence or state being free from defects, deficiencies and significant variations. It is brought about by strict and consistent commitment to certain standards that achieve uniformity of a product in order to satisfy specific customer or user requirements."

The ISO 9000 (1994) defines quality as the degree to which a set of inherent characteristics fulfils requirements. South Africa makes use of the SANS 1200 (1981) and SANS 2001-CC1 (2007)series of Construction Standards (Cidb \& CIDB: South Africa, 2011). Duncan, Thorpe and Sunmer (1990) state that quality refers to standards and the ways and means by which those standards are achieved, maintained and improved upon. Harris and McCaffer (1995) emphasize that quality is meeting the requirements of the customer. According to the International Organization for Standardization (1994) quality is the totality of characteristics of an entity that bear on its ability to meet the stated or implied needs.

Quality control (QC) was defined by Harvey \& Kagan (1990) as the mechanisms of control and the process by which these controls are carried through. Quality control entails checking that all the various stages of the process of serving the customer have been conducted correctly and any defects identified have been corrected (Ayandibu, 2010). According to Zairi (1991), quality control is the operational techniques and activities aimed at both monitoring processes and eliminating causes of unsatisfactory performance of relevant stages of the quality loop in order to result in economic effectiveness. Burke (2007) defines quality control as the method of inspection, in-process inspection and final inspection to confirm that the product has met the required condition.

A study by Farrington \& Ledbetter (1992) analysed the causes of deviations in construction quality. The deviation data was divided into five categories. The five categories are: (1) Design deviations, (2) Construction deviations, (3) Fabrication deviations, (4) Transportation deviations and (5) Operability deviations. Design deviations that were identified by Farrington \& Ledbetter (1992) included design changes in terms of improvements initiated in the design process, construction personnel request, field conditions and owner initiated. Construction deviations consisted of activities that take place at the project site. Deviations that were classified as construction errors are the result of erroneous construction methods and procedures.

\section{Survey starts}

From the investigation, it was determined that there are four categories that lead to poor construction and therefore have an influence on the quality of structural concrete. These four categories are as follows:

- Labour

- Management

- Subcontractors

- Plant and equipment. 


\section{Based on your experience, answer the following questions regarding the four categories that influence the quality of structural concrete}

2. Provide a rating between zero and ten (0-10) for the influence of each Labour attribute on the quality of structural concrete during construction. Zero means no influence and ten means it has an important influence on the quality of structural concrete.

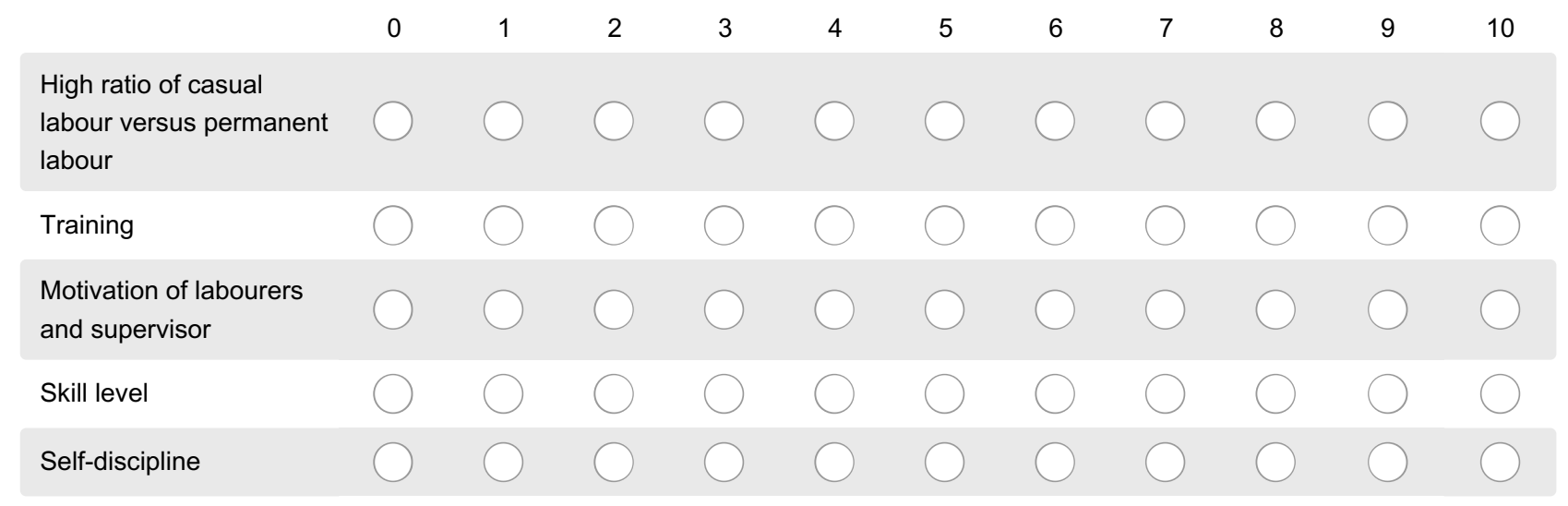

3. Provide a rating between zero and ten (0-10) for the influence of each Management attribute on the quality of structural concrete during construction. Zero means no influence and ten means it has an important influence on the quality of structural concrete. Management is referred to as on-site management.

Coordination
Precision planning
Impractical design
Lack of quality
management expertise
Lack of a Quality
Management System
Poor supervision
Poor communication


4. Provide a rating between zero and ten (0-10) for the influence of Subcontractor attributes on the quality of structural concrete during construction. Zero means no influence and ten means it has an important influence on the quality of structural concrete.

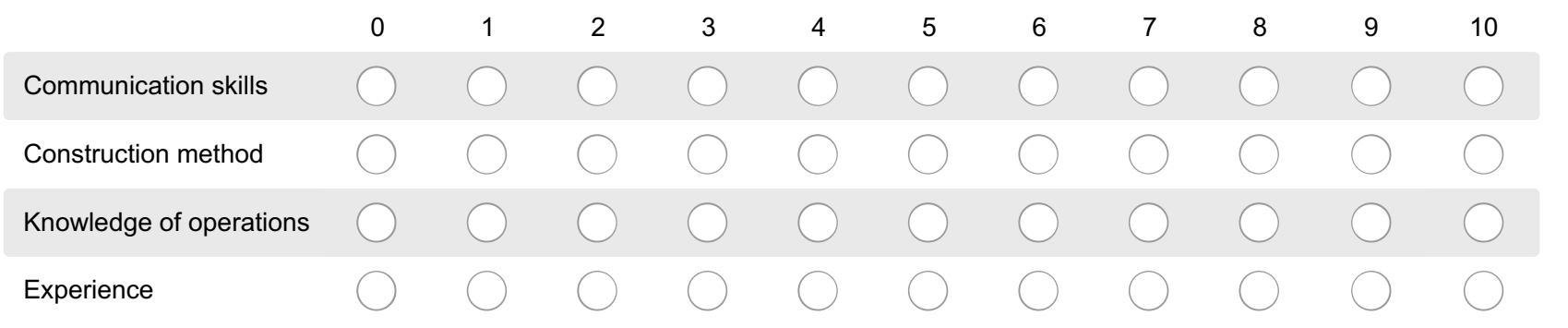

5. Please provide a rating between zero and ten (0-10) for the influence of Plant and Equipment attributes on the quality of structural concrete during construction. Zero means no influence and ten means it has an important influence on the quality of structural concrete.

Equipment that is sub-
standard
Cleanliness of formwork
and shutter boards
Machinery used is old
and poorly maintained

\section{CPD READING continues}

\section{Technological solutions towards monitoring quality aspects}

The purpose of the investigation is to identify a range of technological solutions that could be used to monitor quality inspections on site:

\section{Laser scanners:}

Laser scanners have been used in the industry for condition assessment, component tracking, progress monitoring and construction quality control. Laser scanners are useful to automate data collection. 3D laser scanners are less time-consuming and have higher cost savings compared to the single application of laser scanners (Mani, Feniosky, \& Savarese, 2009). Figure 1 shows an example where laser scanning was used to capture data. 
Use of laser scanners by Cheng and Teizer (2013)

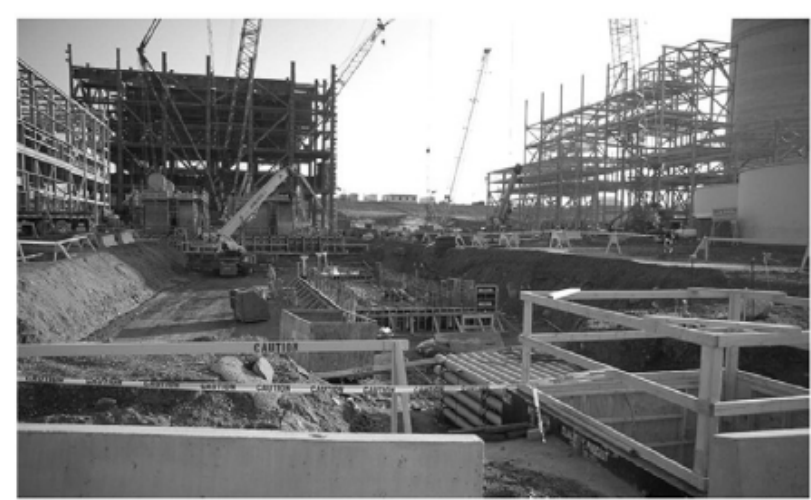

a) Live view of construction site

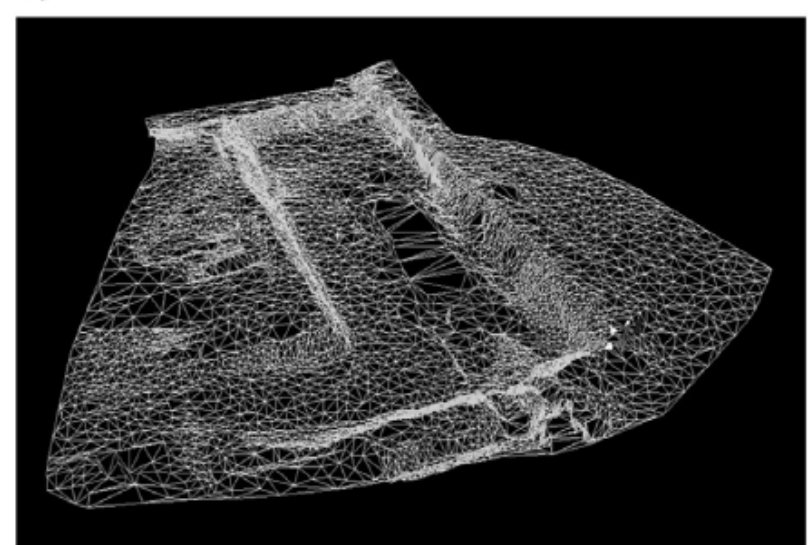

c) Mesh of the terrain

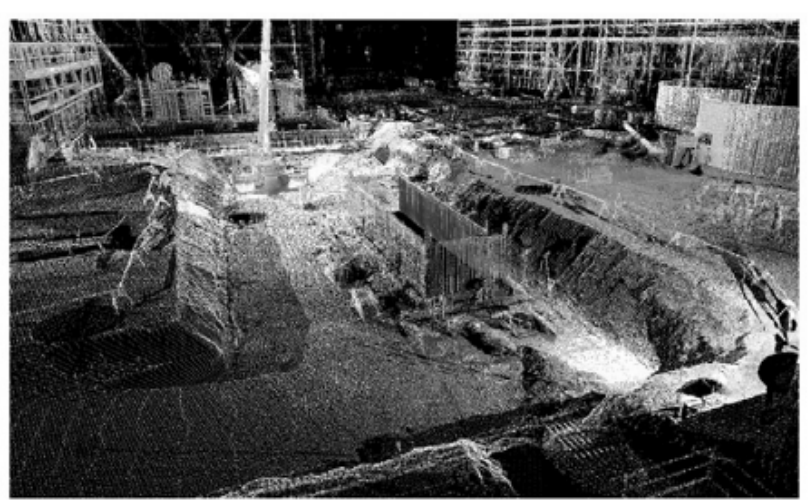

b) Registered point cloud of laser scans

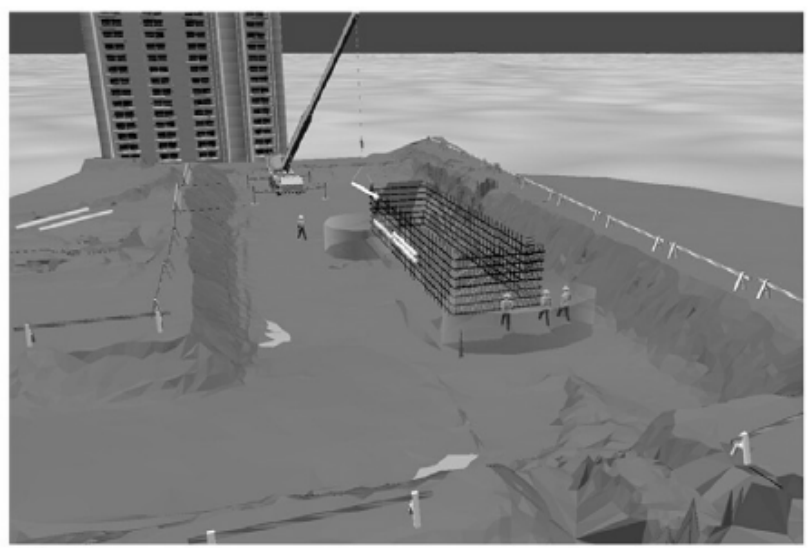

d) Virtual scene including resource models 


\section{Barcodes and RFID tags:}

Barcodes and RFID tags have been used to capture and transmit data from a tag attached to construction products. Jaselskis et al. (1995) identified various potential applications of RFID technology in the construction industry. Possible applications include concrete processing and handling, cost coding for labour and equipment and materials control. Material identification is to identify bulk or discrete solids, liquids, gaseous and primary metals that may or may not be packaged. Parts and containers can be efficiently identified by attaching a tag to each item. This can lead to more efficient material handling and bill-of-materials matching. For construction projects, material identification can reduce confusion regarding arrivals of ordered supplies and their whereabouts at a jobsite.

\section{Photographs on-site:}

Photographs on-site are becoming valuable sources of accurate project information. Digital photos are taken to create a progress photo-log and utilized for coordination and communication. Cameras and photologs have evolved into a significant part of project documentation and thus provide solid participations for their usage as visual and real-time technology which does not require any expertise (Mani et al., 2009). The availability of good quality imagery provides the opportunity for progress monitoring, study of workspace logistics, quality assurance and safety. The photographs enable 3D visualisation of the as-built scene as well as progress data collection. Visualisation models have been adopted into construction to visualize control data, visualize building products and facilitation of constructability.

\section{Other types of technology:}

Other types of technology under consideration include the use of dimensioning software, curing sensors embedded in concrete and quality management software.

The various types of technologies were evaluated by the investigator. It is important to notice that not all the types of quality aspects are influenced equally. Quality aspects have been identified that are influenced by the categories of labour, management, subcontractors and equipment.

\section{Survey continues}

\section{Based on your experience, answer the following questions regarding the influence of each category on the quality aspects:}


6. Please select (from own expert opinion) the influence ofLABOUR on the identified quality aspects for the construction of large footings (for example: footings of wind generators)

Steel spacing incorrect
Compaction incorrect
Grout loss during
pouring
Kncorrect cover obtained
takes place

7. Please select (from own expert opinion) the influence ofMANAGEMENT on the identified quality aspects for the construction of large footings (for example: footings of wind generators)

Bleeding present
Grout loss during
pouring
Incorrect slump and
consistency
Incorrect curing
procedures
Incorrect lap lengths
Correct position of $\mathrm{HD}$
bolts
Pull-out bars incorrect

8. Please select (from own expert opinion) the influence ofSUBCONTRACTORS on the identified quality aspects for the construction of large footings (for example: footings of wind generators)

Incorrect concrete cover
Incorrect steel spacing
$\begin{aligned} & \text { Incorrect slump and } \\ & \text { consistency }\end{aligned}$


9. Please select (from own expert opinion) the influence ofPLANT AND EQUIPMENT on the identified quality aspects for the construction of large footings (for example: footings of wind generators)

Poor finishing
$\begin{aligned} & \text { Incorrect dimensions } \\ & \text { and alignment }\end{aligned}$
$\begin{aligned} & \text { Poor durability } \\ & \begin{array}{l}\text { Delays due to } \\ \text { breakages }\end{array}\end{aligned}$

10. Select (from own expert opinion) the influence ofLABOUR on the identified quality aspects for the construction of other structural elements (for example: beams, columns, slabs)

\begin{tabular}{llll} 
Steel spacing incorrect & Negligible & Minor & Soderate \\
\hline Compaction incorrect & &
\end{tabular}

11. Select (from own expert opinion) the influence ofMANAGEMENT on the identified quality aspects for the construction of other structural elements (for example: beams, columns, slabs)

Bleeding present
Grout loss during
pouring
Incorrect slump and
consistency
Incorrect curing
procedures
Incorrect lap lengths
Position of HD bolts
Pull-out bars incorrect


12. Select (from own expert opinion) the influence ofSUBCONTRACTORS on the identified quality aspects for the construction of other structural elements (for example: beams, columns, slabs)

Incorrect concrete cover
Incorrect steel spacing
$\begin{aligned} & \text { Incorrect slump and } \\ & \text { consistency }\end{aligned}$

13. Select (from own expert opinion) the influence ofPLANT AND EQUIPMENT on the identified quality aspects for the construction of other structural elements (for example: beams, columns, slabs)

\begin{tabular}{|c|c|c|c|c|c|}
\hline & Negligible & Minor & Moderate & Significant & Severe \\
\hline \multicolumn{6}{|l|}{ Poor finishing } \\
\hline \multicolumn{6}{|c|}{$\begin{array}{l}\text { Incorrect dimensions } \\
\text { and alignment }\end{array}$} \\
\hline \multicolumn{6}{|l|}{ Poor durability } \\
\hline $\begin{array}{l}\text { Delays due to } \\
\text { breakages }\end{array}$ & & & & & \\
\hline
\end{tabular}

\section{CPD READING continues}

\section{Cost of rework}

It was stated by Smallwood (2000) that rework constitutes $6 \%$ of project cost for CIDB 5-9 contractors. From these statistics it is evident that inspection is important for achieving higher construction quality (Akinci et al., 2006). Lombard (2011) estimated a rework factor estimate by means of a questionnaire survey. Six consultants and four contractors responded to the questionnaire. From the questionnaire results it was obtained that in South Africa, $15-20 \%$ of total project time is spent in rework and $5-20 \%$ of project cost is spent on rework. Lombard (2011) also stated that $30 \%$ of South African in-situ construction quality does not comply with requirements.

\section{Survey continues}

\section{Based on your experience, answer the following questions}


14. Estimate (from own experience or company information) the expected value of rework on structural concrete as a percentage of the total value of concrete works for the following projects. Also, indicate if this is your own estimate or company data.

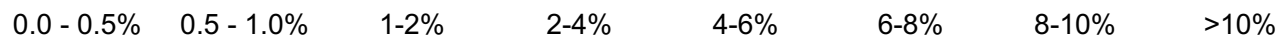

Structural elements (beams, slabs, columns, foundations, walls)

Large footings (wind generators)

Company data or own estimations? 
More questions that were asked in the survey are shown in Figure 11 .

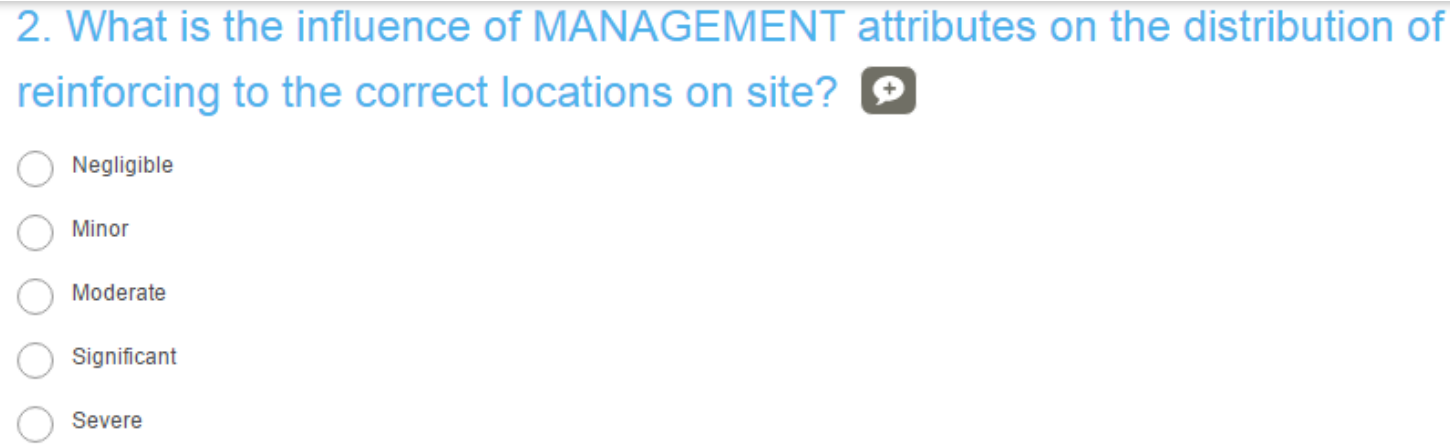

3. In the event that confusion exists regarding the correct location of reinforcing on site, how would you describe the process of solving the problem?

Quickly solved

Moderate wastage of time

Significant wastage of time

Problem could not be solved

4. From personal experience, how often is reinforcement placed in the wrong location on site?
$\bigcirc$ Never
Once in a blue moon
Sometimes
Most of the time
Always

Figure 11: More questions from survey 


\section{J Validation interviews}

Table 9: Opinions obtained from participant 1

\begin{tabular}{cl}
\hline Question number & \multicolumn{1}{c}{ Answer } \\
\hline 1 & $\begin{array}{l}\text { All the important aspects were covered. One factor that also has } \\
\text { a great influence on quality is the standard of materials provided } \\
\text { by the supplier. }\end{array}$ \\
\hline $\begin{array}{l}\text { The critical construction errors have been covered, but cold joints } \\
\text { also lead to excessive rework and should be included. Also, the in- } \\
\text { correct volume of concrete available when pouring columns could } \\
\text { lead to delays due to shortages or excessive wastage due to large } \\
\text { volumes. Large volumes available at pouring could cause concrete } \\
\text { to dry before pouring all the required columns. It is better to } \\
\text { pour concrete from separate batches as it is required. }\end{array}$ \\
$\begin{array}{l}\text { Only data regarding concrete rework in general is available, but } \\
\text { it can be found from non-conformance slips. }\end{array}$ \\
$\begin{array}{l}\text { Top management have meetings and discuss the cost versus the } \\
\text { benefits of the technology. }\end{array}$ \\
$\begin{array}{l}\text { Yes, the tool would be beneficial to identify critical risks before } \\
\text { construction commences. It is useful in the planning phase of a } \\
\text { project. The participant was pleased with the idea and believes } \\
\text { it would also be useful to gain an edge over industry competitors. }\end{array}$ \\
\hline $\begin{array}{l}\text { Yes, but it requires someone to drive the implementation thereof } \\
\text { and someone to whom responsibilities could be assigned to. }\end{array}$ \\
\hline 5 & The younger generation welcomes it, but the older are reluctant \\
& to change.
\end{tabular}

Participant 1 also said that the secondary impacts of rework are even worse which further motivates the use of such a model to reduce rework. Participant 1 suggests that the tool be implemented and the company adjusts the weights and model parameters as it is implemented on various projects.

Table 10 shows the answers from participant 2. 
Table 10: Opinions obtained from participant 2

\begin{tabular}{|c|c|}
\hline Question number & Answer \\
\hline 1 & $\begin{array}{l}\text { The participant commented that the most critical attributes have } \\
\text { been covered, but that the availability of backup equipment can } \\
\text { also be used as an attribute under plant and equipment. }\end{array}$ \\
\hline 2 & $\begin{array}{l}\text { Yes, all the important risks that lead to rework were well covered } \\
\text { and there is nothing critical to add }\end{array}$ \\
\hline 3 & $\begin{array}{l}\text { The company where the participant works, go through extensive } \\
\text { planning before the commencement of projects. They do revise } \\
\text { the data of non-conformances on similar projects before starting } \\
\text { a new project. Therefore, the data could easily be found to assist } \\
\text { in the cost allocation }\end{array}$ \\
\hline 4 & $\begin{array}{l}\text { The company builds spreadsheets of estimations and cost-benefit } \\
\text { analyses before investing in technology. They also make decisions } \\
\text { from qualitative data in the form of presentations done by com- } \\
\text { panies that sell the technology. }\end{array}$ \\
\hline 5 & $\begin{array}{l}\text { Yes, it is a good tool to have. It was also commented that the tool } \\
\text { would serve as a common goal and method of decision making in } \\
\text { the company }\end{array}$ \\
\hline 6 & $\begin{array}{l}\text { Yes, the technologies are innovative and that is a big focus point } \\
\text { for the company. The problem identified with the implementation } \\
\text { thereof is to change the mindset of contractors that are bound to } \\
\text { traditional methods. }\end{array}$ \\
\hline 7 & $\begin{array}{l}\text { There is a general lack of innovation in the construction culture. } \\
\text { Many contractors believe that the implementation of new tech- } \\
\text { nologies are too time consuming and therefore no effort is made } \\
\text { towards the implementation thereof. }\end{array}$ \\
\hline
\end{tabular}

Participant 2 added that such a model would be a good starting point to stimulate the use of technology in the construction industry. The risk analysis provided by the model is also very useful for the management of a company.

The comments from Participant 3 are shown in Table 11

Table 11: Opinions obtained from participant 3

\begin{tabular}{cl}
\hline Question number & \multicolumn{1}{c}{ Answer } \\
\hline 1 & $\begin{array}{l}\text { Yes, the model covers the most important attributes under all the } \\
\text { categories. }\end{array}$ \\
\hline 2 & $\begin{array}{l}\text { Yes, the model covers the critical risks that lead to rework of } \\
\text { structural concrete. }\end{array}$ \\
\hline 3 & $\begin{array}{l}\text { The company only has data readily available of the total rework } \\
\text { costs on projects. }\end{array}$ \\
& $\begin{array}{l}\text { The company makes technological investment decisions based on } \\
\text { the amount of work available. The company also considers the } \\
\text { effectiveness advantage of the technology and how it improves the } \\
\text { production process. However, the company does not have tools } \\
\text { available that quantify the investment advantages. }\end{array}$ \\
\hline 5 & $\begin{array}{l}\text { Yes, it can provide much value by implementing such a system. } \\
\text { Only a change in mindset is required from contractors. }\end{array}$ \\
\hline 7 & $\begin{array}{l}\text { Yes, the technologies would provide good value toward the im- } \\
\text { provement of quality in construction. }\end{array}$ \\
\hline $\begin{array}{l}\text { The younger contractors are innovative and keen to implement } \\
\text { technologies during construction, while the older contractors are } \\
\text { stubborn. }\end{array}$
\end{tabular}


Participant 3 was positive about the implementation of such a decision model and new technologies to improve quality control during construction. The participant believes that in the current economic conditions it would be essential to implement these technological systems to reduce the cost of construction and to stay competitive with the market.

Table 12 shows the comments provided by participant 4 .

Table 12: Opinions obtained from participant 4

\begin{tabular}{cl}
\hline Question number & \multicolumn{1}{c}{ Answer } \\
\hline 1 & Yes, the model covers the important attributes. \\
\hline 2 & $\begin{array}{l}\text { Concerns were raised about the poor quality control of the batch- } \\
\text { ing plant processes to produce concrete. }\end{array}$ \\
\hline 3 & The participant was not aware of such data that is easily available. \\
\hline 5 & $\begin{array}{l}\text { Management considers the current technologies available and how } \\
\text { it improves construction to give the company a leading edge over } \\
\text { its competitors. }\end{array}$ \\
\hline 6 & $\begin{array}{l}\text { Yes, such a model could be used in the tendering process to assist } \\
\text { the tendering team with the cost calculation of the project. }\end{array}$ \\
\hline 7 & $\begin{array}{l}\text { Yes, if the technology is easy to use and the responsibility of using } \\
\text { it could easily be transferred to other team members. }\end{array}$ \\
\hline & $\begin{array}{l}\text { The attitude of management is positive towards the implemen- } \\
\text { tation of technology, but the quality of workforce is too poor to } \\
\text { ensure successful usage of the technology. }\end{array}$ \\
\hline
\end{tabular}

Participant 4 said that such a system could work and he found the concept interesting. However, the participant was not over confident about the implementation of such technologies. The participant was more confident in the risk identification data that is produced through the model.

Table 13 shows the comments from participant 5 .

Table 13: Opinions obtained from participant 5

\begin{tabular}{cl}
\hline Question number & \multicolumn{1}{c}{ Answer } \\
\hline 1 & $\begin{array}{l}\text { The participant added that the planning of equipment availability } \\
\text { should also be included as an important attribute of management. }\end{array}$ \\
\hline 2 & $\begin{array}{l}\text { The participant also suggests better control over batching plants } \\
\text { and ready mix concrete. }\end{array}$ \\
\hline 3 & No, not of the individual construction errors. \\
\hline 4 & $\begin{array}{l}\text { A qualitative approach is followed where management identifies } \\
\text { which technology could be used to outperform competitors. }\end{array}$ \\
\hline 6 & $\begin{array}{l}\text { Yes, such a system would be useful to assist in the planning phase } \\
\text { of a project to determine which teams are best to allocate on } \\
\text { project. Afterwards, the investment decision in technology could } \\
\text { be made. }\end{array}$ \\
\hline 7 & $\begin{array}{l}\text { Yes, the technology can work if it does not consume too much } \\
\text { time during usage. Systems that consume excessive time will not } \\
\text { be used sustainably. }\end{array}$ \\
\hline The implementation of new technology is difficult and the man- \\
agement of such a system would require a newly appointed body \\
that is responsible for it.
\end{tabular}

